\title{
The Ballistic Response of Particle-filled Elastomeric Systems
}

\author{
by \\ Christian Comtois-Arnaldo
}

A thesis submitted to the Faculty of Graduate and Postdoctoral Affairs

in partial fulfillment of the requirements for the degree of

\section{Master of Applied Science}

in

Mechanical Engineering

Carleton University

Ottawa, Ontario

Copyright (C) 2018

Christian Comtois-Arnaldo 
The undersigned recommend to

the Faculty of Graduate Studies and Research

acceptance of the thesis

\title{
The Ballistic Response of Particle-filled Elastomeric Systems
}

\author{
Submitted by Christian Comtois-Arnaldo \\ in partial fulfilment of the requirements for the degree of \\ Master of Applied Science
}

O.E. Petel, Thesis Supervisor

R. Miller, Department Chair

Carleton University

2018 


\section{Abstract}

The present study investigates the complex multiscale ballistic response of particle-filled polymer composites in an effort to link the bulk material behaviour to variations in the microstructure. The multiphase systems under investigation consist of micron-sized ceramic powders integrated within a siloxane elastomer to create flexible particulate composites. In particular, the role of the particulate phase is assessed by varying the filler volume fraction, material, and morphology. The experimental investigation concerns the ballistic penetration of thick targets impacted by 16-grain steel fragment simulating projectiles in the range of $200-900 \mathrm{~m} / \mathrm{s}$. Incident and residual velocities were measured using high-speed videography techniques. Three projectile nose geometries were considered (i.e., flat-nosed, chisel-nosed, and hemispherical) in order to note differences in the ballistic response of composites.

Results are compared to analytical penetration models in order to shed light on the dominant penetration mechanisms and their relationship to the composite microstructure. The ballistic response of neat elastomers and those containing low particle content (e.g., 4.7 vol.\%) was dominated by lateral expansion ahead of the projectile nose. A transition towards an inertia-dominated (plugging) response was observed as the particle content increased to 33.3 vol.\%. The influence of particle density and morphology was quite significant at this volume fraction, whereas signs of extensive interparticle contact were not present.

Particulate composites were characterized under quasi-static tensile and shear conditions as an attempt to correlate the trends with those observed during ballistic penetration. Composites reinforced by irregular-shaped $\mathrm{Al}_{2} \mathrm{O}_{3}$ particles consistently demonstrated enhanced strength and stiffness during quasi-static testing. This trend seemed to translate to its superior ballistic resistance, which may indicate that particle-matrix adhesion strength is an important factor to consider during penetration events. The failure mechanisms are further analyzed through post-mortem imaging of the target surfaces and penetration channels in order to obtain a better understanding of the penetration mechanics involved within particlefilled elastomers. 


\section{Acknowledgments}

Throughout the course of this research project, I have received support from several people and would like to thank each of them for their efforts over the passed couple years. First of all, I would like to thank my supervisor, Prof. Oren Petel, for his guidance and for providing me with a research topic that laid directly in line with my field of interest, i.e., ballistics. I would also like to thank him for exposing me to extensive experimental work while giving me the opportunity to showcase my research at not one, but three conferences. Valuable experience was gained and several memories were made at these events.

I would like to thank DRDC Valcartier and staff, specifically Simon Ouellet, Jacques Blais, and Gilles Pageau, for providing us with access to their facility and performing numerous penetration experiments. The time spent performing experiments at DRDC was truly a highlight of my degree.

I would like to thank Carleton University staff for their technical support throughout the entire project. Specifically, I would like to thank Steve Truttmann for his ongoing interest and willingness to help out on any aspect of my experimental work. Thank you for training me on equipment and helping me solve countless problems. This project could not have been a success without the boys from the machine shop; I would like to thank Alex Proctor, Ian Lloy, and Kevin Sangster for giving me access to the machine shop, manufacturing several dies and projectiles, as well as for teaching me the basics of machining. Thank you for sharing your passion with me. I would also like to thank Marie Tudoret and all previous lab supervisors for allowing a mechanical student to share space in the environmental lab.

I would like to thank my friends who have stayed by my side during this journey and listened to me rant over and over about any issues I encountered. My mental health would be in a much worse state without all of you, so thanks.

Last but not least, I would like to thank my family but especially my parents (Pierre and Maruka Comtois) for everything they have done for me. Thank you for your unconditional love and for always believing in me. Thank you for supporting me in every endeavor I chose 
to pursue and for sharing every emotion with me along the ride. To my dad, thank you for being the ultimate role model. I am forever grateful for the support you gave me over the passed eight years. Finally, I would like to thank my grandmother, Jacqueline Comtois. Thank you for forcing me to practice math as a child. You indirectly led me to engineering and were my prime motivation in pursuing graduate studies, therefore, I dedicate this thesis to you. 


\section{Table of Contents}

$\begin{array}{ll}\text { Abstract } & \text { iii }\end{array}$

Acknowledgments $\quad$ iv

List of Tables $\quad x$

List of Figures $\quad$ xi

Nomenclature $\quad$ xxiv

1 Introduction $\quad 1$

1.1 Rigid Armour Systems . . . . . . . . . . . . . . . . . . . 2

1.2 Soft Armour Systems . . . . . . . . . . . . . . . . . . . 5

1.3 Present Study . . . . . . . . . . . . . . . . . . . . . . . . . . 9

2 Polymer-matrix Composites $\quad 11$

2.1 Polymer Science: Basic Theory . . . . . . . . . . . . . . . . . . . 11

2.1.1 Elastomers . . . . . . . . . . . . . . . . . . 15

2.2 Mechanical Characterization of PMCs: Previous Work . . . . . . . . . . . 17

2.2.1 Fiber-reinforced PMCs: Low-Strain-Rate Loading Conditions . . . . . 18

2.2.2 Particle-reinforced PMCs: Low-Strain-Rate Loading Conditions . . . 23

2.2.3 Particle-reinforced PMCs: Dynamic and Impact Loading Conditions . 27

2.3 Conclusion . . . . . . . . . . . . . . . . . . . 31

3 Terminal Ballistics $\quad 33$

3.1 Penetration of Rigid Projectiles in Semi-infinite Metallic Targets . . . . . . . 35

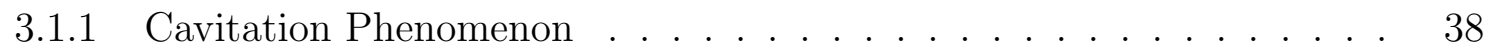

3.1.2 Entrance Phase Effect . . . . . . . . . . . . . . . . . . 41

3.1.3 Role of Projectile and Target Properties on Deep Penetration . . . . 43 
3.2 Analytical Penetration Models of Finite-thickness Metallic Targets . . . . . . 46

3.2.1 Penetration of Metals by Sharp Projectiles . . . . . . . . . . . . . . . 47

3.2.2 Penetration of Metals by Blunt Projectiles . . . . . . . . . . . . . 51

3.3 Dense Particle Suspensions under Ballistic Conditions . . . . . . . . . . . . 58

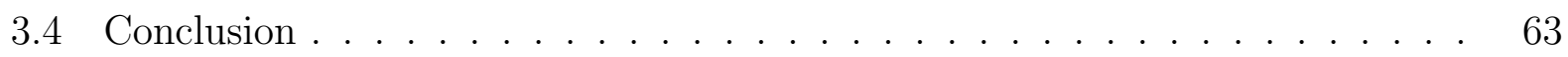

4 Experimental Details $\quad 66$

4.1 Characterization of Ceramic Powders . . . . . . . . . . . . . 66

4.2 Composite Specimen Preparation Methodology . . . . . . . . . . . . 68

4.3 Quasi-static Testing Description and Setup . . . . . . . . . . . . . 69

4.3.1 Shear Testing and Specimen Details . . . . . . . . . . . 70

4.3.2 Tensile Testing and Specimen Details . . . . . . . . . . . 71

4.4 Ballistic Testing Description and Setup . . . . . . . . . . . . . 74

5 Mechanical Characterization: Quasi-static Results and Discussion 76

5.1 Baseline Characterization: Neat PDMS Elastomer . . . . . . . . . . . . 77

5.1 .1 Tensile Testing . . . . . . . . . . . . . . . . 77

5.1 .2 Shear Testing . . . . . . . . . . . . . . . 79

5.2 Influence of Particle Volume Fraction on PDMS Composites . . . . . . . . 81

5.2 .1 Tensile Testing . . . . . . . . . . . . . . . 81

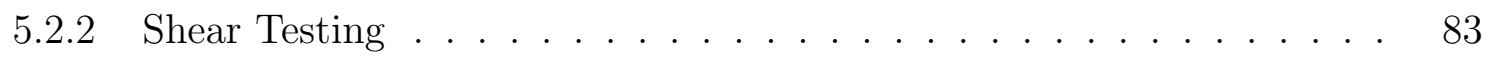

5.3 Influence of Particle Material on PDMS Composites . . . . . . . . . . . . . 84

5.3.1 Tensile Testing . . . . . . . . . . . . . . . 84

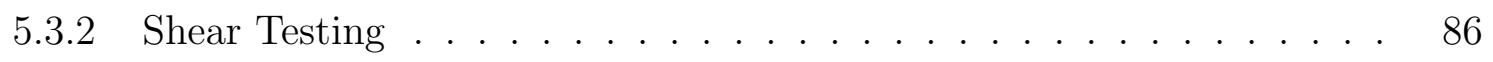

5.4 Influence of Particle Morphology on PDMS Composites . . . . . . . . . . . . 89

5.4 .1 Tensile Testing . . . . . . . . . . . . . . . 89

5.4 .2 Shear Testing . . . . . . . . . . . . . . . . 92

5.5 Quasi-static Testing: Conclusions . . . . . . . . . . . . . . 93 
$5.5 .1 \quad$ Neat PDMS . . . . . . . . . . . . . . . . . . . . . . . . . . . . 93

$5.5 .2 \quad$ Particle Volume Fraction Effect . . . . . . . . . . . . . . . . . . 94

5.5 .3 Particle Material Type Effect . . . . . . . . . . . . . 95

5.5 .4 Particle Morphology Effect . . . . . . . . . . . . . . . . . 95

6 Ballistic Performance Analysis: Results and Discussion 98

6.1 Ballistic Response of PDMS Matrix Penetrated by Various Projectile Nose Geometries . . . . . . . . . . . . . . . . . . . . . . . 99

6.2 Effect of Particle Volume Fraction . . . . . . . . . . . . . . . 105

6.3 Effect of Particle Material Type . . . . . . . . . . . . . . . . . . . . 111

6.3.1 Consideration of Target Areal Density . . . . . . . . . . . . . 115

6.4 Effect of Particle Morphology . . . . . . . . . . . . . . . . . . . . 119

6.4 .1 Effect of Particle Geometry _. . . . . . . . . . . . . 120

6.4 .2 Particle Adhesion Considerations . . . . . . . . . . . . . . . 123

6.5 Projectile Nose Geometry Study . . . . . . . . . . . . . . . . . . . . . 125

6.5.1 Chisel-nosed Projectiles _. . . . . . . . . . . . . . . . 125

6.5.2 Hemispherical Projectiles . . . . . . . . . . . . . . . . . . . 131

6.6 Ballistic Testing: Conclusions . . . . . . . . . . . . . . . . . . . 133

6.6.1 Ballistic Response of PDMS . . . . . . . . . . . . . . . . 134

6.6.2 Effect of Particle Volume Fraction . . . . . . . . . . . . . . . . 134

6.6.3 Effect of Particle Material Type . . . . . . . . . . . . . . . . 135

6.6.4 Effect of Particle Morphology . . . . . . . . . . . . . . . 136

6.6.5 Effect of Projectile Nose Geometry . . . . . . . . . . . . . 136

7 Post-mortem Analysis of Ballistic Capsules 139

7.1 Ballistic Response of PDMS Elastomer . . . . . . . . . . . . . . . . . 139

7.2 Ballistic Response of Particle-filled Elastomers . . . . . . . . . . . . . . . 144

7.2.1 Analysis of Through-thickness Damaged Planes . . . . . . . . . . . 149 
7.2.2 Analysis of Ballistic Penetration Channels . . . . . . . . . . . . 151

8 Conclusion $\quad 156$

$9 \begin{array}{lr}9 \text { References } & 159\end{array}$

$\begin{array}{ll}\text { Appendices } & 173\end{array}$

$\begin{array}{ll}\text { A Ballistic Capsule Testing Results } & 173\end{array}$

B Estimated Ballistic Limit Velocities $\quad 184$

$\begin{array}{ll}\text { C Additional Images } & 185\end{array}$

C.1 Miscellaneous . . . . . . . . . . . . . . . . . . . . 185

C.2 Damaged Planes of Ballistic Capsules . . . . . . . . . . . . . . . . 187

C.3 Penetration Channels of Ballistic Capsules . . . . . . . . . . . . . . . . . 191 


\section{List of Tables}

1 Calculated $V_{\text {cav }}$ values $(\mathrm{km} / \mathrm{s})$ for several rod-target combinations. Table reproduced with permission from Rosenberg and Dekel [86]. . . . . . . . . . . 40

2 Typical mechanical property values of the materials under investigation. . . . 67

3 Particle size distribution data of ceramic powders purchased from Panadyne Inc. . . . . . . . . . . . . . . . . . . . . . 68

4 Summary of tensile testing strength values along with statistical data. Two loading rates, $\dot{\epsilon}: 8.18 \cdot 10^{-3} s^{-1}($ slow $-\mathrm{S})$ and $1.64 s^{-1}$ (fast - F). . . . . . 93

5 Summary of shear testing strength values along with statistical data. Two loading rates, $\dot{\gamma}: 2.13 \cdot 10^{-1} s^{-1}($ slow $-\mathrm{S})$ and $4.92 \cdot 10^{2} s^{-1}$ (fast - F). . . 94

6 Estimated ballistic limit velocities $\left(\mathrm{V}_{b l}\right)$ of baseline PDMS (Syl 184) against various projectile geometries. . . . . . . . . . . . . . . . . . . . . 99

$7 \quad$ Estimated ballistic limit velocities, $V_{b l}(\mathrm{~m} / \mathrm{s})$, for 33.3 vol.\% particulate composites impacted by RCC, chisel-nosed, and hemispherical projectiles. . . . . 129

8 Master ballistic capsule data sheet. . . . . . . . . . . . . . . . . . . . 174

$9 \quad$ Estimated ballistic limit values $\mathrm{V}_{b l}(\mathrm{~m} / \mathrm{s})$ for several target-projectile combi-

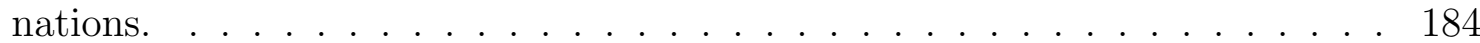




\section{List of Figures}

1 Diagram illustrating evolution of armour systems with time. Figure reproduced with permission from National Research Council [4]. . . . . . . . . . . 1

2 Simulation demonstrating the transverse displacement of fibers within a ballistic fabric during penetration, allowing the projectile to wedge through the openings. Figure reproduced with permission from Cheeseman and Bogetti [32]. 6

3 Schematic of particle-filled elastomeric system (relative sizes not to scale). . . 9

4 Amorphous polymer in (a) relaxed state and (b) strained to the point of partial chain alignment. . . . . . . . . . . . . . . . . . 13

5 Schematic showing influence of temperature and strain rate on tensile response of thermoplastic. . . . . . . . . . . . . . . . . . . . . 15

6 Tensile stress-strain curve for two PDMS elastomers. Figure reproduced with permission from Schneider et al. [57]. . . . . . . . . . . . . . . . 16

7 Schematic of unidirectional continuous fiber-reinforced composite arranged in (a) parallel and (b) series mechanical coupling. . . . . . . . . . . . . . . 19

8 Effect of particle reinforcement on softer matrix. Horizontal lines illustrate the strain distribution within the material when in its (a) relaxed state and (b) strained state. Figure reproduced with permission from McCrum et al. [52]. 20

$9 \quad$ Tensile and shear loads experienced by a perfectly-bonded discontinuous fiber or particulate $\left(a=A R\right.$ and $\left.n \propto \sqrt{G_{m} / E_{f}}\right)$. Figure reproduced with permission from McCrum et al. [52]. . . . . . . . . . . . . . . . .

10 Effect of cylindrical particle aspect ratio $(a=A R)$ on its surface area-tovolume ratio $A / V$. Figure reproduced with permission from McCrum [52]. .

11 Relative tensile strength $\left(\sigma_{c} / \sigma_{m}\right)$ of various particle-filled PMCs, demonstrating the use of upper and lower bound models: + Noryl-talc, $\square$ ABS-talc, $\diamond$ PP-SiC. Figure reproduced with permission from Bigg [70] . . . . . . . . . 
12 Experimental data obtained for 48 vol.\% SiC suspension resulting from plate impact experiments showing: (a) deviatoric stress response $\left(V_{i} \approx 1050 \mathrm{~m} / \mathrm{s}\right)$ and (b) $U_{s}-u_{p}$ Hugoniot curve with separate linear fits for the low- and highvelocity regimes. Figure reproduced with permission from Petel and Higgins

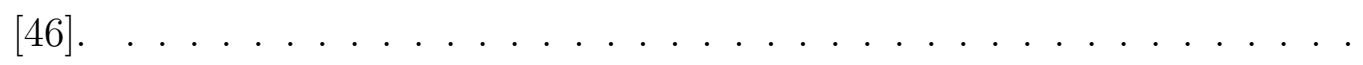

13 Image resulting from a 2D hard-disk model of a 41 vol.\% $\mathrm{SiC}$ suspension experiencing a compressive strain of 0.29. The largest force chain, or cluster of particles, is emphasized. Figure reproduced with permission from Petel et al. $[47] \ldots \ldots \ldots \ldots \ldots \ldots \ldots$

14 Depth of penetration data obtained from a semi-infinite aluminum slab impacted by a rigid ogive-nosed projectile, demonstrating validation of the constant deceleration theory. Figure reproduced with permission from Rosenberg and Dekel $[81] \ldots \ldots \ldots \ldots \ldots \ldots \ldots \ldots$

15 Numerical simulation demonstrating: (a) constant deceleration of ogive projectile, (b) velocity-dependent deceleration of hemisphere projectile. Figures reproduced with permission from Rosenberg and Dekel [86]. . . . . . . . .

16 Numerical simulation showing the effects of target inertia on the deceleration process of (a) hemispherical $\left(V_{i}=1.5 \mathrm{~km} / \mathrm{s}\right)$ and (b) blunt-nosed projectiles $\left(V_{i}=1.0 \mathrm{~km} / \mathrm{s}\right)$. Yield strength of all materials set to $0.4 \mathrm{GPa}$. Figures reproduced with permission from Rosenberg and Dekel [86]. . . . . . . . . . 38

17 Schematic of cavitation phenomenon during the initial penetration stage. Figure reproduced with permission from Hill [87]. . . . . . . . . . . . .

18 Deceleration history of an ogive-nosed rod penetrating an aluminum slab at $1.0 \mathrm{~km} / \mathrm{s}$. Figure reproduced with permission from Rosenberg and Dekel [81]. 42

19 Deceleration history of short ogive-nosed projectiles penetrating aluminum slabs. Figure reproduced with permission from Rosenberg and Dekel [89]. . . 
20 Numerical simulations of penetration depth results of rigid rods with various nose shapes into semi-infinite aluminum targets. Figures reproduced with permission from Rosenberg and Dekel [86]. . . . . . . . . . . . . . .

21 Numerical simulation of deceleration history of a conical-nosed tungsten projectile penetrating $\left(V_{i}=800 \mathrm{~m} / \mathrm{s}\right)$ : (a) semi-infinite and finite-thickness aluminum targets and (b) targets of varying thickness. Figures reproduced with permission from Rosenberg and Dekel [81]. . . . . . . . . . . . . . . 47

22 A variety of failure mechanisms found in finite targets resulting from different impact events. Figure taken from Rosenberg and Dekel [81]. . . . . . . . .

23 Failure mechanisms in a $10 \mathrm{~mm}$ thick 2014-T6 aluminum alloy penetrated by (a) ogive, (b) hemispherical, and (c) blunt projectiles. Figures reproduced

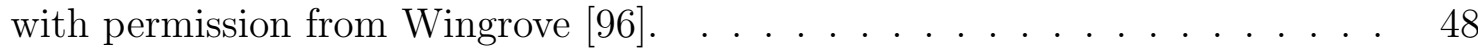

24 Example of ductile hole expansion resulting from conical projectile perforating a mild steel plate. Figure reproduced with permission from Woodward [98]. .

25 Analysis of numerical simulation results showing the normalized effective resisting stress for (a) thin to intermediate thickness plates $(H / D \leqslant 1)$ and (b) thick plates $(H / D \geqslant 1)$. Figures reproduced with permission from Rosenberg and Dekel $[99] . \ldots \ldots \ldots \ldots$

26 Numerical simulations showing the transition in failure mechanisms of targets having varying thicknesses $(H / D=0.25,0.3$, and 0.5$)$ impacted by a projectile. Figure reproduced with permission from Rosenberg and Dekel [99]. 51

27 Ductile shear plug formation within a steel plate, resulting in a shear dimpled surface. Figure reproduced with permission from Borvik et al. [101]. . . . . . 54

28 Example of adiabatic shear bands nucleating and coalescing, thus forming a plug within a titanium alloy. Figure reproduced with permission from Woodward $[106] \ldots \ldots \ldots \ldots \ldots \ldots \ldots$ 
29 Schematic of penetration resistance transitions between ductile hole expansion and adiabatic shearing. Figure reproduced with permission from Woodward $[107$

30 Difference in plugging response obtained from (a) an aluminum alloy and (b) a high-purity aluminum. Voids were found to nucleate at alloying elements. Figure reproduced with permission from Woodward [102] . . . . . . . . . . 56

31 Schematic of ballistic penetration experiments on STF-filled capsules. Figure reproduced with permission from Petel et al. [112]. . . . . . . . . . . . 58

32 Normalized velocity data and RI plugging model for 21 SiC-EG mixture. Figure reproduced with permission from Petel et al. [44] . . . . . . . . . . . . . 59

33 Normalized velocity results of two particle suspensions, exposing the effect of volume fraction on ballistic resistance. Figure reproduced with permission from Petel et al. [44]. . . . . . . . . . . . . . . . .

34 Average resistive shear stress of several dense particle suspensions impacted by chisel-nosed projectiles. Figure reproduced with permission from Petel et

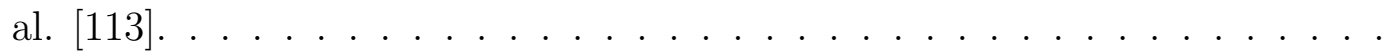

35 SEM images of powders: (a) boron carbide $\left(\mathrm{B}_{4} \mathrm{C}\right)$, (b) silicon carbide ( $\left.\mathrm{SiC}\right)$, (c) diamond , (d) alumina $\left(\mathrm{Al}_{2} \mathrm{O}_{3}\right)$ - irregular, and (e) alumina $\left(\mathrm{Al}_{2} \mathrm{O}_{3}\right)$ -

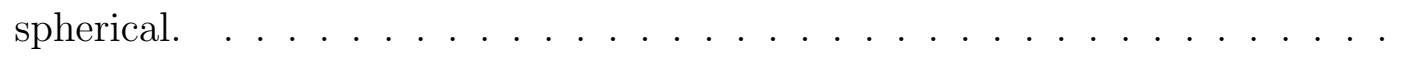

36 Steel shear punch tool: (a) open die showing punch with mounted specimen and (b) closed-die assembly. Apparatus manufactured according to ASTM D732-10 [115]. . . . . . . . . . . . . . . . . . . 70

37 Aluminum shear sample mould - designed according to ASTM D732-10 [115]. 70

38 MTS and apparatus mounting setup for shear punch testing. . . . . . . . . . 72

39 Aluminum tensile sheet mould. . . . . . . . . . . . . . . . . . . 73

40 Steel rule dumbbell cutting die - manufactured by Key Dies Inc. . . . . . . . 73 
41 Tensile experiment setup: (a) dumbbell specimen with support tabs and (b) MTS configuration. . . . . . . . . . . . . . . . 73

42 Single-stage smooth-bore light gas gun (DRDC Valcartier) . . . . . . . . . . 74

43 Experimental setup for ballistic testing: (a) schematic and (b) photograph. . 74

44 Various 16-grain steel fragment simulating projectiles tested in the present study. ................................. 75

45 Tensile stress-strain curves of the PDMS elastomer given two curing conditions $\left(\dot{\epsilon}=8.18 \cdot 10^{-3} s^{-1}\right) . \ldots \ldots \ldots \ldots \ldots$

46 Tensile stress-strain curves of heat-cured PDMS loaded at two strain rates $\dot{\epsilon} . \quad 79$

47 Shear results for neat PDMS demonstrating the effect of loading rate for two curing conditions: (a) room temperature cure (RTC) and (b) heat cure (HC). 80

48 Shear results for neat PDMS demonstrating effect of cure temperature at two punch velocities: (a) $V_{p}=1.3 \mathrm{~mm} / \mathrm{min}$ and (b) $V_{p}=3000 \mathrm{~mm} / \mathrm{min}$. . . . . . 81

49 Tensile results showing the effect of particle volume fraction for $\mathrm{Al}_{2} \mathrm{O}_{3}$-PDMS $(\mathrm{HC})-\dot{\epsilon}=8.18 \cdot 10^{-3} s^{-1} \ldots \ldots \ldots \ldots 1$

50 Relative tensile strength versus particle volume fraction for $\mathrm{Al}_{2} \mathrm{O}_{3}$-PDMS composites. . . . . . . . . . . . . . . . . . 82

51 Shear results showing volume fraction effect for SiC-PDMS (RTC) loaded bottom-side-first. . . . . . . . . . . . . . . . . 83

52 Shear strength of RTC composite versus particle ( $\mathrm{SiC}$ ) volume fraction. . . . 84

53 Tensile stress-strain curves for 33.3 vol.\% SiC- and $\mathrm{Al}_{2} \mathrm{O}_{3}$-PDMS (HC) composites, showing effect of material type $\left(\dot{\epsilon}=8.18 \cdot 10^{-3} s^{-1}\right) . \ldots . . . \quad . \quad 85$

54 Tensile stress-strain curves demonstrating strain rate-sensitivity of 33.3 vol.\% particulate composites: (a) $\mathrm{SiC}$ and (b) $\mathrm{Al}_{2} \mathrm{O}_{3} \ldots \ldots \ldots \ldots$

55 Shear results showing the effect of particle material types (slow rate). . . . . 87 
56 Shear results for $33.3 \% \mathrm{Al}_{2} \mathrm{O}_{3}$-PDMS (RTC) - strain-rate sensitivity study (slow: $\mathrm{V}_{p}=1.3 \mathrm{~mm} / \mathrm{min}$ or $\dot{\gamma} \approx 2.13 \cdot 10^{-1} \mathrm{~s}^{-1}$, fast: $\mathrm{V}_{p}=3000 \mathrm{~mm} / \mathrm{min}$ or $\left.\dot{\gamma} \approx 4.92 \cdot 10^{2} s^{-1}\right) \ldots \ldots \ldots \ldots \ldots \ldots$

57 Shear results for (a) $33.3 \% \mathrm{Al}_{2} \mathrm{O}_{3}$-PDMS - effect of cure temperature and (b) 33.3\% SiC- and $\mathrm{Al}_{2} \mathrm{O}_{3}$-PDMS (HC) composites. . . . . . . . . . . . . 89

58 Tensile results showing effect of particle morphology $-\dot{\epsilon}=8.18 \cdot 10^{-3} s^{-1}$. . $\quad 90$

$5933.3 \%$ spherical $\mathrm{Al}_{2} \mathrm{O}_{3}$-PDMS tensile failure surface showing particle-matrix debonding: a) 500x and b) 2kx. . . . . . . . . . . . . . . . 91

60 Shear results of $\mathrm{Al}_{2} \mathrm{O}_{3}$-PDMS (HC) composites - effect of particle morphology. 92

$61 V_{r}-V_{i}$ curves for neat PDMS impacted by different projectile geometries: (a) RCC, (b) chisel, (c) hemisphere, and (d) overall. . . . . . . . . . . . . . . . 100

62 Schematic of lateral expansion with cavity formation ahead of RCC projectile nose. Arrows signify direction of material motion. Red circles demonstrate the diminished contact area between projectile and target. . . . . . . . . . . 101

63 Theoretical RI perforation and plugging models for neat PDMS. . . . . . . . 101

$64 \mathrm{~V}_{b l}$-normalized residual velocity data of neat PDMS impacted by various projectiles along with respective RI models. . . . . . . . . . . . . . . . . . . . . 102

65 Post-penetration images of neat PDMS impacted by different projectiles: (a) $\operatorname{RCC}\left(V_{i}=522 \mathrm{~m} / \mathrm{s}\right),(\mathrm{b})$ chisel-nosed $\left(V_{i}=501 \mathrm{~m} / \mathrm{s}\right)$, and $(\mathrm{c})$ hemispherical $\left(V_{i}=469 \mathrm{~m} / \mathrm{s}\right) \ldots \ldots \ldots \ldots \ldots \ldots \ldots$

66 Normalized ballistic performance parameter results for neat PDMS impacted by RCC and chisel-nosed projectiles. . . . . . . . . . . . . . . . 104

67 Volume fraction effect for SiC-PDMS composites impacted by RCC projectiles: (a) $V_{r}-V_{i}$ curves and (b) normalized performance parameter. . . . . . 106

68 Volume fraction effect for $\mathrm{B}_{4} \mathrm{C}-\mathrm{PDMS}$ composites impacted by $\mathrm{RCC}$ projectiles: (a) $V_{r}-V_{i}$ curves and (b) normalized performance parameter. . . . . . 106 
69 Volume fraction effect for Diamond-PDMS composites impacted by RCC projectiles: (a) $V_{r}-V_{i}$ curves and (b) normalized performance parameter. . . . 107

70 Post-penetration images of RCC projectiles impacting slightly above $V_{b l}$ for (a) neat PDMS at $t=t_{0}$, (b) neat PDMS at $t=t_{1}$, (c) 4.7 vol. $\%$ diamondPDMS at $t=t_{0}$, and (d) 4.7 vol.\% diamond-PDMS at $t=t_{1} \ldots \ldots$. . . . 107

71 Schematic of idealized shear plugging event between particulate composite and RCC projectile. Arrows illustrate the restriction of lateral motion along with axial acceleration of the material ahead of projectile nose. Dashed red line shows shock wave traveling ahead of projectile and compressing material. 108

72 Post-penetration images $\left(\mathrm{V}_{i} \approx 900 \mathrm{~m} / \mathrm{s}\right)$ of SiC-PDMS at various volume fractions: (a) 0 vol.\% - neat PDMS, (b) 4.7 vol.\%, (c) 23.6 vol.\%, and (d) 33.3 vol.\%. . . . . . . . . . . . . . . . . . . . . 109

73 Estimated $V_{b l}$ values for composites having varying particle volume fractions. 109

$74 \mathrm{~V}_{b l}$-normalized residual velocity data with corresponding RI plugging models demonstrating volume fraction effect for: (a) SiC-, (b) $\mathrm{B}_{4} \mathrm{C}-$, and (c) diamondPDMS composites. ....................... . . . . . . . . . . . . . .

75 Plugging fraction parameter demonstrating volume fraction effect for various composites impacted by RCC projectiles: (a) $\phi_{f}=4.7 \%$, (b) $\phi_{f}=23.6 \%$,

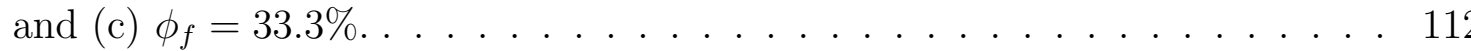

76 Effect of particle material type for 33.3 vol\% composites impacted by RCC projectiles: (a) $V_{r}-V_{i}$ curves and (b) normalized performance parameter. . . 113

77 Ballistic limit velocity versus composite (33.3 vol.\%) density - projectile: RCC.114

78 Plugging response for 33.3 vol.\% composites impacted by RCC projectiles: (a) $\mathrm{V}_{b l}$-normalized residual velocity data with corresponding $\mathrm{RI}$ plugging models and (b) plugging fraction parameter. . . . . . . . . . . . . . 116

79 Constant areal density study (chisel-nosed projectiles): (a) $V_{r}-V_{i}$ curves and (b) work required for penetration. . . . . . . . . . . . . . . . 117 
80 Constant areal density study (chisel-nosed projectiles): average resistive shear stress of 33.3 vol.\% composite targets. . . . . . . . . . . . . . . . . . . 118

81 Particle morphology study with 33.3 vol. $\% \mathrm{Al}_{2} \mathrm{O}_{3}-\mathrm{PDMS}$ impacted by RCC projectiles: $V_{r}-V_{i}$ curves. . . . . . . . . . . . . . . . . . 119

82 Particle morphology study with 33.3 vol.\% $\mathrm{Al}_{2} \mathrm{O}_{3}-\mathrm{PDMS}$ impacted by RCC projectiles: (a) $\mathrm{V}_{b l}$-normalized residual velocity curves and (b) average resistive shear stress. . . . . . . . . . . . . . . . . . . . . . . 120

83 Comparison of 33.3 vol.\% irregular SiC-PDMS and spherical $\mathrm{Al}_{2} \mathrm{O}_{3}$-PDMS quasi-static response: (a) tension and (b) shear. . . . . . . . . . . . . . 122

84 Comparison of ballistic results for 33.3 vol.\% irregular $\mathrm{Al}_{2} \mathrm{O}_{3}$-PDMS, SiCPDMS, and spherical $\mathrm{Al}_{2} \mathrm{O}_{3}$-PDMS composites: (a) $V_{r}-V_{i}$, RCC, (b) $V_{r}-V_{i}$, chisel-nosed, (c) $\tau_{a v}$, RCC, and (d) $\tau_{a v}$, chisel-nosed. . . . . . . . . . . . . 123

85 Relative tensile strength of various particulate composites along with particle adhesion models. . . . . . . . . . . . . . . . . . . . 124

86 Volume fraction effect for SiC-PDMS impacted by chisel-nosed projectiles: (a) $V_{r}-V_{i}$ curves and (b) normalized performance parameter. . . . . . . . . 126

87 Volume fraction effect for SiC-PDMS impacted by chisel-nosed projectiles: (a) $\mathrm{V}_{b l}$-normalized residual velocity data with $\mathrm{RI}$ plugging models and (b) plugging fraction parameter. . . . . . . . . . . . . . . . . 127

88 Projectile nose geometry effect on 4.7 vol.\% SiC-PDMS: $V_{r}-V_{i}$ cuves. . . . 127

89 Estimated ballistic limit velocity of SiC-PDMS composites having varying particle volume fraction impacted by RCC and chisel-nosed projectiles. . . . 128

90 Effect of projectile nose shape for two 33.3 vol.\% composites: (a) $V_{r}-V_{i}$ curves and (b) normalized performance parameter. . . . . . . . . . . . . . . 128

$91 V_{b l}$-normalized residual velocity curves for 33.3 vol.\% particulate composites impacted by RCC and chisel-nosed projectiles. Data correlated to RI plugging models: (a) SiC-PDMS and (b) $\mathrm{Al}_{2} \mathrm{O}_{3}$-PDMS 
$92 V_{b l}$-normalized residual velocity curves and corresponding RI plugging models for 33.3 vol.\% SiC- and $\mathrm{Al}_{2} \mathrm{O}_{3}$-PDMS impacted by: (a) chisel-nosed and (b)

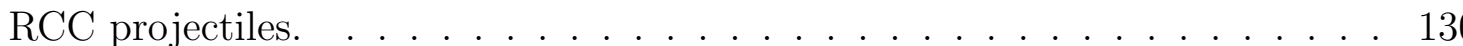

93 Projectile nose geometry effect for 33.3 vol.\% SiC- and $\mathrm{Al}_{2} \mathrm{O}_{3}$-PDMS systems: average resistive shear stress. . . . . . . . . . . . . . . . . . . 131

94 Ballistic results showing effect of particle integration against penetration of hemispherical projectiles. . . . . . . . . . . . . . . . . . 132

95 Residual velocity curves for 33.3 vol.\% particulate composites impacted by hemispherical and RCC projectiles: (a) SiC-PDMS and (b) $\mathrm{Al}_{2} \mathrm{O}_{3}$-PDMS. . .

96 Correlation with plugging models: (a) $V_{b l}$-normalized residual velocity curves for 33.3vol.\% particulate composites impacted by hemispherical and RCC projectiles and (b) plugging fraction parameter for composites impacted by hemispherical projectiles. . . . . . . . . . . . . . . . . .

97 Image of neat PDMS impacted by chisel-nosed projectile at impact velocities (a) slightly below and (b) slightly above $V_{b l}$. . . . . . . . . . . . . . . . 140

98 Images of neat PDMS penetrated by RCC projectiles, demonstrating transition in failure mechanism throughout penetration channel (shear to tensile failure): (a) $V_{i}=214 \mathrm{~m} / \mathrm{s}$, (b) $V_{i}=300 \mathrm{~m} / \mathrm{s}$, and (c) isometric view, $V_{i}=300$ $\mathrm{m} / \mathrm{s} \ldots \ldots \ldots \ldots \ldots \ldots \ldots \ldots \ldots \ldots$

99 Image of neat PDMS impacted by hemispherical projectiles near $V_{b l}$, showing a single tensile crack failure mechanism. . . . . . . . . . . . . . . . . . . 142

100 Images of neat PDMS capsules impacted by RCC projectiles at: (a) $V_{i}=345$ $\mathrm{m} / \mathrm{s},(\mathrm{b}) V_{i}=435 \mathrm{~m} / \mathrm{s},(\mathrm{c}) V_{i}=613 \mathrm{~m} / \mathrm{s}$, and (d) $V_{i}=888 \mathrm{~m} / \mathrm{s}$. Results show the progression of radial cracking with increasing velocity. . . . . . . . . . . 143 
101 (a) Post-penetration image of neat PDMS impact surface. Radial cracks propagate from each corner of a chisel-nosed projectile. The projectile corners act as stress concentrations, which initiate cracks during penetration. The cracks then grow due to excessive lateral expansion (cavitation). (b) Top and side view of chisel-nosed projectile. . . . . . . . . . . . . . . . . 143

102 SEM images showing a portion of penetration channels of SiC-PDMS at different volume fractions (arrows indicate penetration direction): (a) 4.7 vol.\% SiC-PDMS - at impact surface, (b) 4.7 vol.\% SiC-PDMS - strands, (c) 33.3 vol.\% SiC-PDMS - midway through capsule thickness, and (d) 33.3 vol.\% SiC -strands. . . . . . . . . . . . . . . . . . . .

103 Schematic demonstrating the development of planar cracks through tensile fracture, resulting in discing failure. Figure reproduced with permission from Woodward [118]. . . . . . . . . . . . . . . . . . . 147

104 Schematic demonstrating the development of planar cracks through shear fracture, resulting in discing failure. Figure reproduced with permission from Woodward $[106] . \ldots \ldots \ldots \ldots \ldots \ldots$

105 SEM images showing formation of radial cracks: (a) 4.7 vol.\% SiC-PDMS impact surface (b) 33.3 vol.\% SiC-PDMS - mid-thickness surface. Penetration direction: into the page. . . . . . . . . . . . . . . . . 148

106 Schematic illustrating the proposed failure mechanism during penetration of particulate composites: (a) planar tensile fracture may occur at the projectiletarget edges while shear fracture may form ahead of nose due to bending (orange lines represent cracks) during penetration; (b) strands return to their original position upon decompression. Arrows indicate direction of motion. . 149

107 Stitched images showing entire penetration channel of 33.3 vol. $\% \mathrm{Al}_{2} \mathrm{O}_{3}$ -

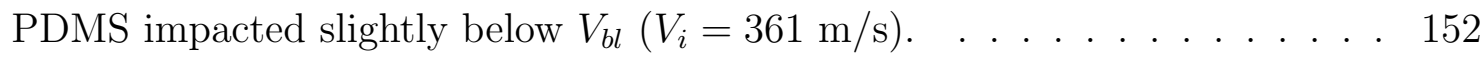


108 Trapped shear plug that was extracted from 4.7 vol.\% SiC-PDMS capsule impacted by RCC at $V_{i}=257 \mathrm{~m} / \mathrm{s}$. The plug diameter (approximately 2.5

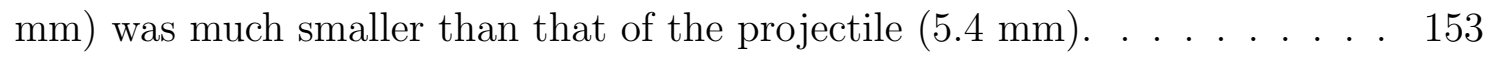

109 Tensile failure surface (specimen corner) of $33.3 \%$ spherical $\mathrm{Al}_{2} \mathrm{O}_{3}-\mathrm{PDMS}$ showing matching interfaces along with cluster of nanoparticles (red circle). . 185

110 Ballistic performance parameter for PDMS and EG capsules. Data for EG was extracted from Petel et al. [44]. . . . . . . . . . . . . . . . . 186

111 SEM images of 33.3 vol.\% spherical $\mathrm{Al}_{2} \mathrm{O}_{3}$-PDMS penetration channel: (a) overall and (b) magnification. . . . . . . . . . . . . . . . . . . 186

112 Damaged planes - 4.7 vol.\% SiC-PDMS (projectile: $\mathrm{RCC}$ ) $-V_{i}=257 \mathrm{~m} / \mathrm{s}$ (slightly above $V_{b l}$ ): (a) impact surface, (b) through-thickness surface $t_{r e l}=$ $0.33,(\mathrm{c}) t_{r e l}=0.67$, and $(\mathrm{d})$ back surface. Direction of impact: into the page except for $(\mathrm{d}) \ldots \ldots \ldots \ldots \ldots \ldots$. . . . . . . . . . . . . . . . . . . . . . . . .

113 Damaged planes -4.7 vol.\% SiC-PDMS (projectile: chisel-nosed) $-V_{i}=229$ m/s (slightly above $V_{b l}$ ): (a) impact surface, (b) through-thickness surface $t_{r e l}=0.33,(\mathrm{c}) t_{r e l}=0.67$, and $(\mathrm{d})$ back surface. Direction of impact: into the page except for $(\mathrm{d}) \ldots \ldots \ldots \ldots \ldots \ldots$. . . . . . . . . . . . . . . . . . . . . . . .

114 Damaged planes -4.7 vol.\% SiC-PDMS (projectile: $\mathrm{RCC}$ ) $-V_{i}=430 \mathrm{~m} / \mathrm{s}$ : (a) impact surface, (b) through-thickness surface $t_{r e l}=0.33$, (c) $t_{r e l}=0.67$, and (d) back surface. Direction of impact: into the page except for (d). . . 188

115 Damaged planes - 33.3 vol.\% SiC-PDMS (projectile: RCC) $-V_{i}=423 \mathrm{~m} / \mathrm{s}$ : (a) impact surface, (b) through-thickness surface $t_{\text {rel }}=0.33$, (c) $t_{\text {rel }}=0.67$, and (d) back surface. Direction of impact: into the page except for (d). . . 188

116 Damaged planes -33.3 vol.\% SiC-PDMS (projectile: $\mathrm{RCC}$ ) $-V_{i}=740 \mathrm{~m} / \mathrm{s}$ : (a) impact surface, (b) through-thickness surface $t_{r e l}=0.33$, (c) $t_{r e l}=0.67$, and (d) back surface. Direction of impact: into the page except for $(\mathrm{d})$. . . . 189 
117 Damaged planes - 33.3 vol. $\% \mathrm{Al}_{2} \mathrm{O}_{3}$-PDMS (projectile: $\mathrm{RCC}$ ) $-V_{i}=711 \mathrm{~m} / \mathrm{s}$ : (a) impact surface, (b) through-thickness surface $t_{\text {rel }}=0.33$, (c) $t_{\text {rel }}=0.67$, and (d) back surface. Direction of impact: into the page except for $(\mathrm{d}) . \quad \ldots \quad 189$

118 Damaged planes - 33.3 vol.\% SiC-PDMS (projectile: chisel-nosed) $-V_{i}=392$ m/s: (a) impact surface, (b) through-thickness surface $t_{r e l}=0.33$, (c) $t_{r e l}=$ 0.67, and (d) back surface. Direction of impact: into the page except for $(d)$.

119 Damaged planes - 33.3 vol.\% SiC-PDMS (projectile: hemispherical) $-V_{i}=$ $414 \mathrm{~m} / \mathrm{s}$ : (a) impact surface, (b) through-thickness surface $t_{r e l}=0.33$, (c) $t_{r e l}=0.67$, and $(\mathrm{d})$ back surface. Direction of impact: into the page except

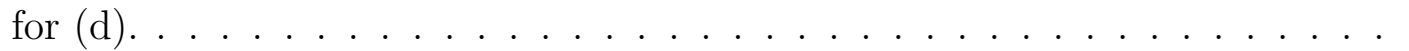

120 Stitched images of entire penetration channels of 4.7 vol.\% SiC-PDMS capsules (projectile: RCC) showing effect of impact velocity: (a) $V_{i}=257 \mathrm{~m} / \mathrm{s}$ and (b) $V_{i}=430 \mathrm{~m} / \mathrm{s}$. Impact and exit surfaces at top and bottom of images,

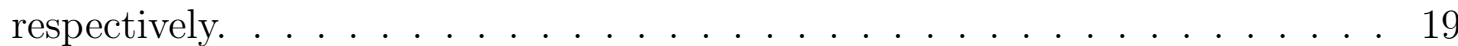

121 Stitched images of entire penetration channels of SiC-PDMS capsules (projectile: RCC) showing effect of particle volume fraction: (a) $V_{i}=430 \mathrm{~m} / \mathrm{s}$ and (b) $V_{i}=423 \mathrm{~m} / \mathrm{s}$. Impact and exit surfaces at top and bottom of images, respectively. . . . . . . . . . . . . . . . . . .

122 Stitched images of entire penetration channels of 33.3 vol.\% SiC-PDMS capsules (projectile: RCC) showing effect of impact velocity: (a) $V_{i}=423 \mathrm{~m} / \mathrm{s}$ and (b) $V_{i}=740 \mathrm{~m} / \mathrm{s}$. Impact and exit surfaces at top and bottom of images, respectively. . . . . . . . . . . . . . . . . . . .

123 Stitched images of entire penetration channels of 33.3 vol.\% particulate composites (projectile: RCC) showing effect of particle material type: (a) $\mathrm{SiC}$ PDMS, $V_{i}=740 \mathrm{~m} / \mathrm{s}$ and (b) $\mathrm{Al}_{2} \mathrm{O}_{3}-\mathrm{PDMS}, V_{i}=711 \mathrm{~m} / \mathrm{s}$. Impact and exit surfaces at top and bottom of images, respectively. 
124 Stitched images of entire penetration channels of 33.3 vol.\% SiC-PDMS capsules impacted by various projectile nose geometries: (a) RCC, $V_{i}=423 \mathrm{~m} / \mathrm{s}$, (b) chisel-nosed, $V_{i}=392 \mathrm{~m} / \mathrm{s}$, and (c) hemispherical, $V_{i}=414 \mathrm{~m} / \mathrm{s}$. Impact and exit surfaces at top and bottom of images, respectively. . . . . . . . 195 


\section{Nomenclature}

$\mathrm{SiC} \quad$ Silicon carbide

$\mathrm{B}_{4} \mathrm{C} \quad$ Boron carbide

$\mathrm{Al}_{2} \mathrm{O}_{3} \quad$ Aluminum oxide

MMC Metal-matrix composite

PMC Polymer-matrix composite

SHPB Split-Hopkinson pressure bar

STF Shear-thickening fluid

DPS Dense particle suspension

PDMS Polydimethylsiloxane

FSP Fragment simulating projectile

$\phi_{f} \quad$ Filler volume fraction (vol.\%)

$\rho \quad$ Density

$\sigma_{c} \quad$ Composite strength

$\sigma_{m} \quad$ Matrix strength

E Young's (elastic) modulus

$E_{f} \quad$ Filler elastic modulus

$E_{m} \quad$ Matrix elastic modulus

$A R \quad$ Aspect ratio

$G_{m} \quad$ Matrix shear modulus

$A / V \quad$ Filler surface area-to-volume ratio

$\tau_{m} \quad$ Shear strength of polymer matrix

$V_{i} \quad$ Impact (or incident) velocity

D Projectile diameter

$V_{\text {cav }} \quad$ Critical velocity for cavitation

DHE Ductile hole enlargement

$V_{r} \quad$ Residual (or exit) velocity 
$V_{b l} \quad$ Ballistic limit velocity

$W_{p} \quad$ Penetration work of projectile

RI Recht and Ipson

$H / D \quad$ Plate thickness relative to projectile diameter

$E_{f} \quad$ Deformation energy due to plastic impact

$W_{s} \quad$ Shear work expended during penetration

$\beta \quad$ Mass ratio between ideal plug and projectile

$A_{r} \quad$ Cross-sectional area ratio between plug and projectile

ASB Adiabatic shear bands

EG Ethylene glycol

$\mathrm{SiO}_{2} \quad$ Silica (Silicon dioxide)

$\kappa \quad$ Normalized performance parameter

$\tau_{a v} \quad$ Average resistive shear stress

DRDC Defence Research and Development Canada

MTS Material Testing System

SEM Scanning electron microscopy

HC Heat-cured

RTC Room-temperature-cured

ASTM American Society for Testing and Materials

$V_{p} \quad$ Shear punch displacement rate

$\dot{\gamma} \quad$ Shear strain rate

$\dot{\epsilon} \quad$ Tensile strain rate

fps $\quad$ Frames-per-second

RCC Right-angled circular cylinder projectile

$\epsilon_{\text {uts }} \quad$ Strain-to-failure (tension)

Syl 184 Sylgard 184 (Dow Corning)

BSF Bottom-side-first orientation 
TSF Top-side-first orientation

$p_{f} \quad$ Plugging fraction parameter

$A_{d} \quad$ Areal density

$t_{\text {rel }} \quad$ Relative target thickness 


\section{Introduction}

Conflict has been a recurring issue throughout human history, which has led to the increased demand for proper protection systems. The field of ballistic protective equipment requires the ongoing development of new materials and armour systems in order to better protect personnel (e.g., military soldiers, law enforcement officers, etc.) from existing or emerging ballistic threats. Aside from the inherent protective requirements, the weight and mobility of ballistic personnel armours are of primary concern $[1,2]$ while also considering potential cost constraints. The development of novel composite materials has become the focus of much research in order to improve upon existing armours [3, 4]. Figure 1 illustrates the evolution of armour systems with time in terms of decreasing weight (or areal density).

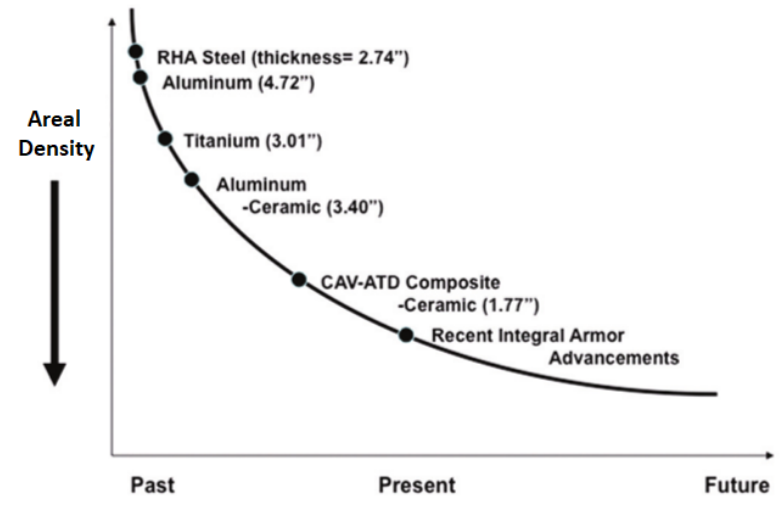

Figure 1. Diagram illustrating evolution of armour systems with time. Figure reproduced with permission from National Research Council [4].

When designing a new armour system that is dedicated to the protection of personnel against a specific threat, armour developers are tasked to propose a combination of ballistic materials layered in a configuration that achieves the most effective protection while minimizing weight. This requires ballistic performance data of individual materials, which is ideally obtained from an existing dataset library. Alternatively, a new armour material must be conceptualized and thoroughly evaluated prior to its introduction into an armour system. The reader is referred to a book prepared by the National Research Council for further discussion on the process of armour development [4]. 
The viability of potential armour materials is assessed through a combination of techniques. Impact and penetration experiments are typically conducted over a range of velocities to collect raw data for a specific ballistic event, providing some fundamental understanding of the material behaviour. Analytical models derived from fundamental conservation laws and assumptions concerning specific failure mechanisms are often used to shed light on the penetration process. These models can complement numerical simulations of the impact event, which are used to gain more information on the deformation process and the stress field during penetration [5]. In order to resolve complex ballistic scenarios, a combination of the three methods is suggested.

\subsection{Rigid Armour Systems}

Classical investigations have dealt with the penetration response of metallic plates due to their general use and their popularity for armour systems. Furthermore, metals offer a wide range of mechanical properties and failure mechanisms. In particular, steels have been used in many defense applications since they have high strength and good ductility, are relatively inexpensive, and are readily available. The comparatively high density of steel however restricts its use for lightweight applications.

Various lightweight ceramics such as silicon carbide $(\mathrm{SiC})$, boron carbide $\left(\mathrm{B}_{4} \mathrm{C}\right)$, and aluminum oxide $\left(\mathrm{Al}_{2} \mathrm{O}_{3}\right)$ have been used for personnel armour, specifically as add-on plates for torso protection against more energetic munitions. Ceramics are often laminated with metallic backing-plates (e.g., aluminum), resulting in significant weight reduction for the same level of protection obtained from steel plates [2]. The purpose of ceramic plates is to fracture or at least erode the incoming projectiles during penetration, thus, drastically minimizing their ballistic effectiveness. The metallic backing plate serves to absorb the remainder of the kinetic energy left in the fragments through plastic deformation and momentum transfer mechanisms $[6,7]$.

Although the variability in mechanical properties for metals and ceramics is quite exten- 
sive, their material response can be further modified through the integration of reinforcement filler materials such as ceramic particles, fibers, or whiskers. Particulates have been integrated into numerous materials throughout history in order to provide the designer with the ability to modify and fine-tune their mechanical, thermal, optical, or conductive properties for a given application. A two-phase system may be formed through chemical interactions between the matrix and the filler material, which creates a bonded interface that can be thought of as a third phase [8]. Many composite parameters can influence the response of the system, such as filler volume fraction, shape and aspect ratio, size, surface chemistry, hardness and strength, as well as density. The role played by particles during the penetration of a multiphase composite depends on the choice of matrix as well as the loading condition and strain rate; therefore, it is important to understand how their interactions with the surrounding matrix and the penetrator cause a change in their associated defeat mechanisms.

Chin [7] claimed that metal-matrix composites (MMCs) consisting of ceramic particles were becoming an affordable material candidate for applications such as aerospace structural and high-temperature engine components, as well as for armour defense systems. Ceramic particles were found to increase the dynamic flow stress and strain-rate sensitivity of a metal under high-strain-rate compression $[9,10]$ and demonstrated a significant influence resulting from particle aspect ratio and shape. Karamis et al. [11] qualitatively investigated the ballistic performance of Al-SiC MMCs from a tribological stand-point and concluded that the particles increased the friction between the MMC and projectile, thus, dissipating kinetic energy and reducing the penetration depth. On the other hand, the inclusion of ceramic particles in ductile aluminum matrices was found to decrease their impact toughness, resulting in brittle fracture [12]. These particles introduced microstructure defects such as particle agglomeration, which established preferential crack initiation and propagation sites.

The addition of particulate fillers were found to also benefit ceramics by improving their inherently low fracture toughness. The enhanced toughness results from the interaction 
between propagating cracks and the particulate phase. The particles serve to impede and arrest further crack growth within brittle materials [13]. The interested reader is referred to a review article on ceramic-matrix composites for more information [14].

Alternative lower cost armour solutions include polymer-matrix composites, or PMCs. Arias et al. [3] integrated $\mathrm{Al}_{2} \mathrm{O}_{3}$ particles into a vinylester resin at 50 vol.\%. These tiles were combined with an aluminum backing-plate and offered intermediate ballistic efficiency against an armour-piercing projectile. Although they were not as effective as monolithic ceramic/aluminum armours, these could be manufactured at a fraction of the cost. This study demonstrates that integrating reinforcing filler materials may provide low cost armour alternatives, designed for specific threats.

Particle-reinforced PMCs have been extensively studied due to the ability of particles to drastically influence the overall behaviour of the polymer [15]. The primary scope of research in this field focuses on quasi-static characterization $[16,17]$, dynamic fracture testing $[18,19$, 20], and high-strain-rate compression experiments using plate-impact methods [21, 22, 23] or split Hopkinson pressure bars (SHPB) [24]. There are very limited studies such as the one from Arias et al. [3] that investigate the ballistic penetration behaviour of these PMCs, thus, warranting further research to understand the role of particulates during such highspeed events.

The effect imparted by the addition of filler material into one polymer matrix does not necessarily translate to the same response found in another and requires experimentation. For example, the tensile response of a glassy polyurethane was barely altered by the addition of silica particles, however, a significant increase in all aspects was observed when the same particles were added to an elastomeric polyurethane [25]. The role of particles consisted of reducing the mobility of the polymer chains, therefore, the varying response of materials to particle integration was attributed to different degrees of cross-linking density found in the polymers. On the other hand, the dynamic compressive strength of PMCs having different matrices was found to converge to relatively similar values as the particle volume fraction 
was increased to $50 \%$. This may indicate the possibility that particle-particle interactions are beginning to dominate the material response at high strain rates and pressures [24].

Polymers are often reinforced by integrating continuous fillers such as high-performance fibers, which have revolutionized the field of lightweight armour systems. Common examples of these fibers include aramids (Kevlar, Twaron), ultra-high molecular weight polyethylene fibers (Spectra, Dyneema), glass fibers, and more. These can be integrated into a polymer matrix as unidirectional layers or woven into a ballistic fabric. The general use of high-performance fibers in ballistic products has been justified by their mechanical properties, which can be characterized by their high strength-to-weight ratio and high energy absorption capabilities. Consequently, fiber-reinforced PMCs have been thoroughly studied under ballistic penetration conditions and have found applications in military helmets and lightweight vehicle armour alternatives [26].

The energy-absorption mechanisms present during the penetration of fiber-reinforced PMCs have been studied by several authors $[27,28,29,30,31]$ and depend on various parameters such as the choice of materials, projectile geometry and velocity, boundary conditions, as well as plate thickness and laminate configuration. A few examples of common energy-absorption mechanisms include initial compression, matrix failure, tensile and shear failure of fibers, delamination, and global bending. A thorough review on the penetration of fiber-reinforced composites is provided by Cheeseman and Bogetti [32].

\subsection{Soft Armour Systems}

High performance fibers are commonly woven into fabrics and layered upon each other without the use of a binding matrix, resulting in relatively flexible ballistic vests for personnel protection against low to medium energy projectiles. Upon projectile impact, woven fabrics absorb the kinetic energy through plastic deformation and dissipate it throughout the surrounding network of fibers [1]. The ballistic response of these fabrics cannot be predicted solely based on fiber properties, but rather a combination of that and the weave structure 
[33]. Projectiles have a greater tendency to wedge themselves through loosely woven fabrics since the individual fibers are able to deflect transversely upon penetration, resulting in lower fiber density ahead of the projectile nose (e.g., see Fig. 2). The occurrence of this phenomenon is favored if the penetrator size is relatively small and/or impacts on an angle. The projectile could then slip through the opening by displacing the fibers instead of further damaging them [32]. This fiber spacing enlargement process is not only a function of fabric structure, but also depends on projectile geometry and fiber mobility. Frictional effects arising from the interaction of fibers and even between the yarns of each fiber influence the overall mobility of the fibers. This inter- and intra-fiber friction was found to play an important role in the penetration mechanics of woven fabrics $[34,35]$. Without the existence of friction, a small penetrator would be capable of navigating itself through the thickness of the fabric by simply displacing the fibers laterally along its path until penetration is complete. Friction limits this event and once again promotes plastic deformation of the fibers [32], which is a more effective energy-absorption mechanism.

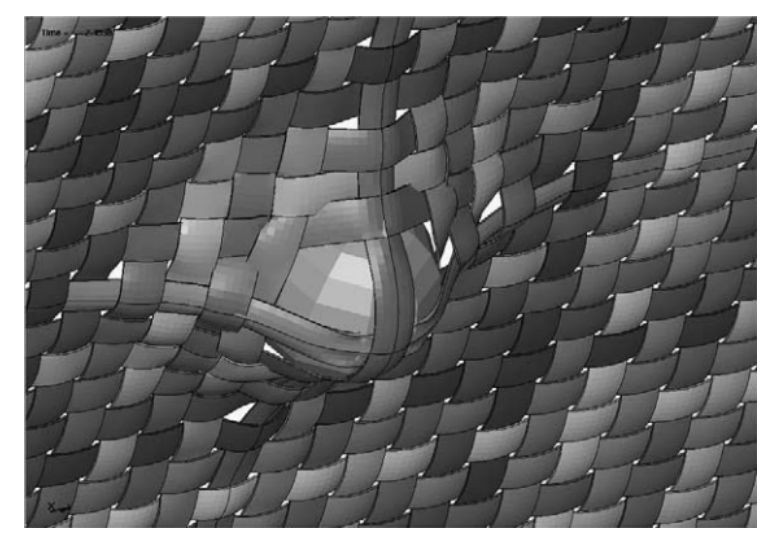

Figure 2. Simulation demonstrating the transverse displacement of fibers within a ballistic fabric during penetration, allowing the projectile to wedge through the openings. Figure reproduced with permission from Cheeseman and Bogetti [32].

Similarly, Lee et al. [36] demonstrated that fiber mobility could be constrained by introducing a low amount of resin into a fabric, which promoted increased energy absorption as the fibers were strained to failure. Although the resin matrix did not absorb a significant amount of energy, it had several indirect contributions. For example, the coupling of fibers 
was achieved since stresses were uniformly distributed throughout the penetration region, hence raising the required load to failure. Additionally, an increased number of fibers were strained to failure, which resulted from decreased fiber slippage. These effects are expected to be analogous to the contribution of fiber friction.

Ballistic fabrics are currently the primary flexible (or soft) personnel armour system in use, although several different fiber materials and configurations exist. Researchers have been devising methods to alter their response for decades. Fabric systems have been previously modified by integrating shear-thickening fluids (STFs) into the weaves as an interstitial to increase fiber friction, thus, creating a fluid-armour system. This was first reported by Gates Jr. in 1968 [37] and was consequently patented in 1972 [38]. A shear-thickening fluid can be described as a dense suspension of particles within a liquid matrix that exhibits a non-Newtonian increase in viscosity at high strain rates. The rheological phenomenon has been described as a discontinuous jump in viscosity that occurs once a certain shear rate threshold is surpassed. This is partially attributed to the formation of a network of particle clusters that temporarily resist motion within the liquid [39]. Such systems have recently regained interest over the past few decades, resulting in numerous studies on STF-modified fabrics thereafter $[40,41,42]$ as well as some review articles, e.g., [43]. Ballistic fabrics impregnated by STFs were deemed capable of decreasing armour thickness while providing the same level of protection against low energy projectiles, thus, improving flexibility and mobility [40]. Some research has found that these systems displayed limited performance contribution when impacted by hard projectiles at high velocities [37, 44], although they showed promise for spike and stab protection $[41,45]$.

The shear-thickening phenomenon was claimed to be responsible for the enhanced ballistic performance of fabrics, however, these rheology studies were conducted at much lower stresses and strain rates than those found during ballistic penetration. Extrapolation of such behaviour to high strain rates and pressures should be avoided. The increased interest of STF-coated fabrics for ballistic protection has resulted in further investigations on the dy- 
namic behaviour of dense particle suspensions (DPS). Therefore, plate-impact experiments were performed on such fluids to simulate the extreme dynamic states involved in ballistic events [46]. Suspensions having a sufficiently high particle content experienced a sharp rise in dynamic strength when loaded above a certain threshold velocity. It was suggested that the relative compressibility between the liquid and solid phases could be causing a local increase in particle volume fraction upon impact, resulting in interparticle contact. This mechanism was described as being analogous to the classical shear thickening of DPS. Numerical simulations showed that a network of force chains capable of carrying load was formed [39, 47], thus demonstrating evidence of shear strength and a diminishing contribution from the liquid matrix.

Aside from wetted systems, ballistic studies have also been conducted on fabrics coated with dry ceramic powders. Particle integration was claimed to improve the energy distribution capabilities of a fabric by increasing the inter-fiber friction [35, 45, 48, 49, 50]. Weight addition was therefore minimized by eliminating the need for a liquid matrix without compromising its performance relative to the fluidized system [45].

In terms of real-life applications, these modified fabric systems may suffer from particle suspension or containment issues over time and would require some sort of sealant material to enclose the added mass. A solution for this problem could be to polymerize the particulate system using an elastomeric matrix in order to maintain its flexibility. Particle-filled elastomers could be integrated as an interstitial within ballistic fabrics in order to cover specific areas of interest. Polymerization of the particulate system would eliminate the particlesuspension and containment issues associated to STF- and dry particle-impregnated fabrics; however, proper characterization of the composite behaviour and an evaluation of its ballistic performance is required for system optimization. 


\subsection{Present Study}

In order to expand on the development and modification of soft armour materials, the present study investigates the ballistic behaviour of a polydimethylsiloxane (PDMS) elastomer doped with several micron-sized ceramic particles to create flexible composites having varying inclusion properties. The interest of such material is based on its potential to help stop fine fragments from penetrating through existing fabric systems by transverse fiber-displacement mechanisms (Fig. 2), while retaining overall flexibility. The microstructure of the system can be described as a network of long and flexible polymer chains that are cross-linked and further constrained by the inclusion of hard particles, as illustrated by the simplified schematic in Fig. 3. Although there are several ballistic studies concerning polymer composites reinforced by continuous fibers (e.g., $[28,29,30,51]$ ), the potential ballistic benefits of particle-reinforced polymers have been investigated to a lesser extent. Furthermore, research in this field has been primarily focused on rigid polymer matrices. As an initial attempt to fill this knowledge gap, the objective of the present study is to determine the role of the particulate phase during the ballistic penetration of an elastomeric composite. The performance of numerous composites has been evaluated, while identifying their dominant penetration mechanisms and noting any variations resulting from changes in the microstructure. In particular, composites have been prepared in order to assess their ballistic behaviour according to variations in particle volume fraction, filler material, density, and morphology, thus enabling future system optimization.

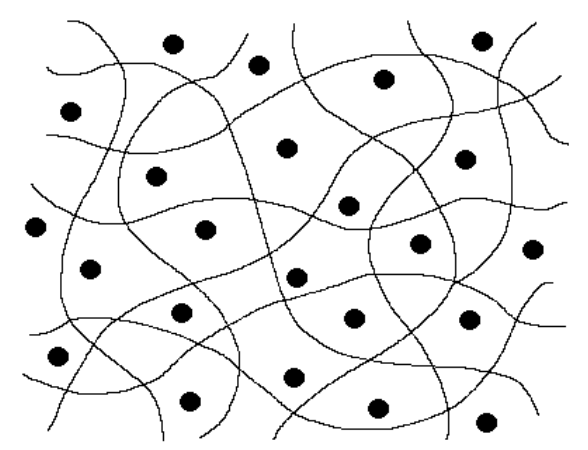

Figure 3. Schematic of particle-filled elastomeric system (relative sizes not to scale). 
Ballistic experiments were conducted using a single-stage smooth bore light gas gun. Cylindrical targets were penetrated by 16-grain steel fragment simulating projectiles (FSPs) that were accelerated to velocities ranging from 200 to $900 \mathrm{~m} / \mathrm{s}$. Incident and residual velocities were measured using high-speed videography techniques. The projectile nose shape was varied while maintaining a constant mass to determine the influence of penetrator geometry on the material behaviour and its defeat mechanisms. The variations in dynamic response observed during experimentation are qualitatively explained and the velocity data are compared to analytical penetration models. This has allowed the author to shed light on the dominant penetration mechanisms of the composites and their relationship to the microstructure. In order to complement the ballistic results, composites were characterized under quasi-static tension and shear conditions in an attempt to demonstrate the emerging trends in behaviour resulting from the contribution of different filler materials; a limited discussion on the strain-rate sensitivity of particle-filled elastomer systems is provided.

Proper development of the composite described above could result in a flexible material having dynamic strengthening mechanisms associated to interparticle contacts as was observed in DPS [46] and rigid PMCs [24]. The present study shall investigate the occurrence of such phenomenon. Regardless, the characterization of particle-filled elastomers provides new integration opportunities for armour developers, thus, broadening their options during the material selection stage. 


\section{Polymer-matrix Composites}

Prior to analyzing the quasi-static and ballistic behaviour of our particle-reinforced elastomers, a general review of the research done on polymer-matrix composites is in order. The present discussion is focused on the analytical methods and models that researchers have used to predict or characterize the material response under quasi-static and dynamic loading conditions. In particular, the influence of particle inclusions is investigated.

Integration of particulates within polymeric materials offers the potential to dramatically improve mechanical properties. A popular example of this is the introduction of carbon black fillers into rubber, which provides the required durability for their use in vehicle tire applications. In order to attain the desired improvements, it is important to understand how the filler and matrix interact with each other and how modifications to the material

response can be made. An improper composite design could result in adverse effects such as a significant loss of material strength or ductility. The contribution of filler material depends on the loading condition and rate. The improvements obtained during quasi-static experiments do not necessarily translate to the same trends when subjected to impact, therefore it is crucial to understand the role that particles play within the system. There are many particulate parameters that can influence the composite behaviour, e.g., particle size, shape, concentration, strength, surface chemistry, etc. The present chapter provides a limited discussion concerning studies on the effect of such parameters, however a brief overview on polymer science is provided first. Due to this large field of study, only the most relevant subjects shall be discussed. A specific focus on the response of elastomeric materials is also included, considering the thesis subject at hand.

\subsection{Polymer Science: Basic Theory}

A polymer is composed of long molecular chains consisting of several repeating units (i.e., monomers) that are joined together by covalent bonds at their extremities [52, 53]. For 
example, polymerization of a simple ethylene $\left(\mathrm{C}_{2} \mathrm{H}_{4}\right)$ monomer results in the breakage of its double bond so that it can attach itself to neighboring monomers. This occurs consecutively, resulting in the formation of long molecular chains, i.e., polyethylene. The length of molecular chains is an important parameter that governs the mechanical properties of the polymer and is described by its molecular weight. A single molecular chain is capable of spanning over a large portion of the polymer, therefore exerting its influence on a large volume of material. This is the primary factor that distinguishes polymers from metals and ceramics [52].

Polymers have been classified based on the interactions between their molecular chains. A thermoset polymer is one whose chains have been chemically bonded (i.e., cross-linked) together at tie points along their length. This chemical reaction results from the introduction of a cross-linking (or curing) agent consisting of monomers that have a higher degree of functionality. Several polymer chains are joined at each tie point, forming a large interconnected molecular network. Heat is often required to initiate cross-linking, although some thermosets can be cured at room temperature. Once these chemical bonds are formed, the natural (or stable) shape of the polymer is set [52]. Thermosets do not melt at elevated temperatures, although their molecular network can deteriorate. The polymer can be deformed under stress, however upon relaxation, the cross-links act to recover its stable shape. Since the components used to synthesize thermosets are initially low viscosity liquids, these types of polymers have found common use in the development of composites. These liquids allow for simple and effective integration of filler materials and can be moulded into a large variety of shapes. Although the resulting composites can have several issues, they have large economic advantages for low-volume production runs [54].

Thermoplastics are composed of individual polymer chains that are blended (or tangled) together, but not chemically linked. When sufficiently heated, the molecules are able to separate and flow like a viscous liquid due to the lack of cross-linking within the system. The molecules are then frozen into their new positions once cooled to room temperature. 
Thermoplastics are more suitable for large production runs due to the machinery required during the manufacturing process. Their advantage comes from the fact that only heat and pressure must be applied to form parts, i.e., no curing agents are required [54].

For a given chemical composition, the physical arrangement (i.e., structure) of the molecular chains within a polymer has an influence on its macroscopic properties. The arrangement of a molecular chain with respect to itself (rotational isomerism) is one aspect that must be considered, however it will not be further discussed. The second consideration comes from the arrangement of the chains with respect to each other, i.e., the degree of crystallinity and the orientation of the chains. Several polymers have an amorphous configuration. The molecular chains are disordered, having no apparent structure [53]. Two noteworthy types of amorphous polymers include glasses and elastomers. Some degree of order can be obtained when amorphous polymers are subjected to significant strain. The polymer chains are able to preferentially align themselves in the direction of applied stress [52], as seen in Fig. 4.

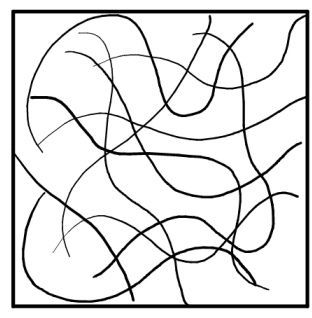

(a)

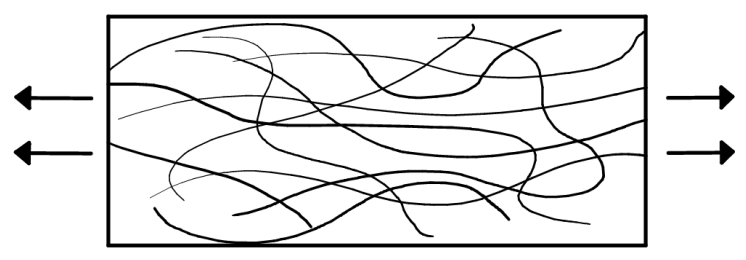

(a)

Figure 4. Amorphous polymer in (a) relaxed state and (b) strained to the point of partial chain alignment.

Polymer structures can also exhibit significant order, forming crystals of highly oriented chain segments within the melt. The mutual arrangement of these chains aims to minimize the Gibbs free energy within the system [52]. These types of polymers are known as crystalline polymers. Crystallization within a polymer melt can occur if molecules have a regular structure, the temperature is below the crystal melting point, and sufficient time is allowed for the long chains to order themselves [53]. Crystals within a polymer are highly anisotropic. Monomers within a molecular chain are covalently bonded while neighboring 
chains are held together by weaker secondary bonds [52]. Therefore, the direction of loading strongly affects the mechanical properties of the polymers. The Young's modulus reaches its highest value when strained along the direction of the molecular chains. This is also true for amorphous polymers whose chains have been temporarily aligned due to stretching of the material, as in Fig. 4. Although crystalline polymers may have a high degree of crystallinity, partly amorphous regions that separate the crystals still exist within the system; in reality, these should be called semi-crystalline polymers. The fringed-micelle model was the first and simplest model to illustrate the structure of a semi-crystalline polymer. This model has now been deemed unrealistic, however the central fact remains true: molecular chains pass through several crystalline and amorphous regions within the two-phase solid and are therefore pinned together along their entire length [52]. Longer molecular chains are more effective at this pinning mechanism, which consequently improves polymer strength.

The mechanical behaviour of a given type of material can be described through general equations that correlate their stress and strain, which are known as constitutive relations. The simplest example of such relation is Hooke's law $(\sigma=E \epsilon)$, which describes the behaviour of an ideal elastic solid under uniaxial deformation. This equation states that the strain $(\epsilon)$ is linearly proportional to the stress $(\sigma)$ of a solid and has a slope equal to its elastic modulus $(E)$. This equation is based on the small-strain elasticity theory and implies that the solids have no temperature or strain-rate dependence [53]. In contrast, polymers are typically quite sensitive to these parameters and behave in a non-linear manner at high strain. The constitutive relations used to describe polymers should therefore account for this behaviour.

Polymers are generally categorized as viscoelastic materials, i.e., exhibiting an intermediate behaviour between a viscous liquid and an elastic solid [53]. An important polymer property is the glass-transition temperature $\left(T_{g}\right)$, which dictates the degree of mobility between polymer chains at a certain temperature. Polymers consisting of short molecular chains behave as a viscous fluid when $T>T_{g}$ since the chains are highly mobile. On the other hand, polymers having long molecular chains exhibit a rubber-like behaviour. When 
the temperature drops below $T_{g}$, the molecules are frozen into position [52]. The temperature and strain rate sensitivity of polymers is clearly illustrated in Fig. 5. At low temperatures or high strain rates, a given polymer may demonstrate a glassy behaviour, consisting of a relatively high Young's modulus and low strain-to-failure (curve $A$ ). At high temperatures or low strain rates, the same polymer could behave as a rubber, having a low Young's modulus and capable of withstanding large deformations along with full recovery upon relaxation (curve $B)[53]$.

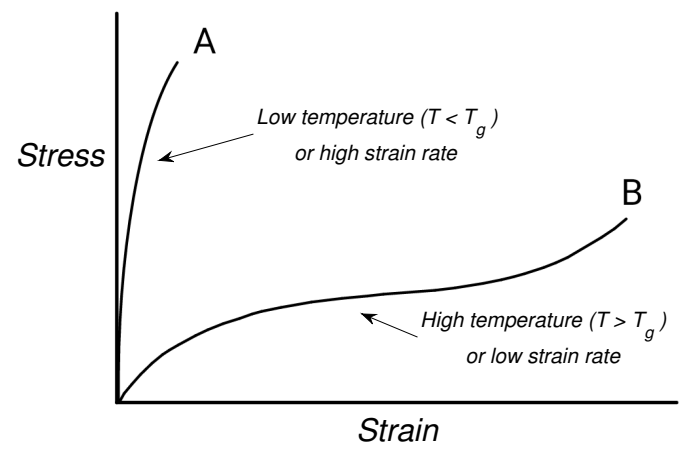

Figure 5. Schematic showing influence of temperature and strain rate on tensile response of thermoplastic.

\subsubsection{Elastomers}

An elastomer is an amorphous polymer whose flexible molecular chains are cross-linked together by covalent bonds, resulting in extremely high extensibility while exhibiting complete recovery after deformation $[52,55]$. The $T_{g}$ of elastomers is lower than room temperature, hence their highly elastic nature. The benefits of cross-linking were first discovered by adding sulfur into natural rubber (polyisoprene), which significantly improved its overall properties and resulted in its characteristic elastic recovery. The cross-linking density of a polymer can be directly correlated to its stiffness [52].

The mechanical properties of PDMS elastomers have been investigated due to their relevance in several different applications. Examples of such applications include microelec- 
tromechanical systems [56, 57], microfluidic devices [58, 59, 60], and soft lithography [61]. Sylgard 184 was designated as the matrix of choice for the present thesis due to reasons that will be mentioned in Chapter 4; consequently, this PDMS will be used as an example for discussion of elastomeric behaviour. Previous researchers have performed uniaxial tension experiments on Sylgard 184 in order to extract the elastic modulus, ultimate tensile strength, and strain-to-failure values. A decently large range of values have been reported $[62,58,56,63]$, although the trends found in the stress-strain curves are similar. An example of such curve for two PDMS elastomers is shown in Fig. 6 [57]. The tensile behaviour of these elastomers can be described as having a linear stress-strain relationship up to strain values of approximately 45\%, allowing for the calculation of Young's modulus through Hooke's law. A non-linear strain-hardening trend follows upon further extension prior to ultimate failure, which is likely attributable to the temporary alignment of polymer chains. These stress-strain curves have the same characteristics as the conceptual curve $B$ found in Fig. 5, which exemplifies a thermoplastic loaded above its $T_{g}$ value.

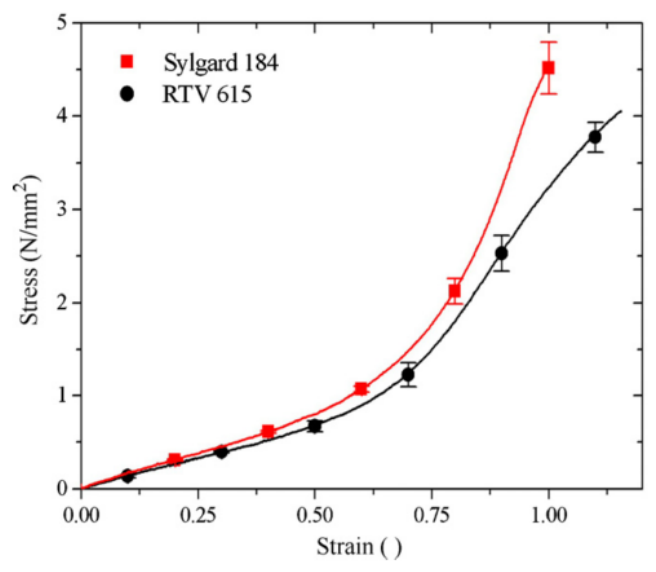

Figure 6. Tensile stress-strain curve for two PDMS elastomers. Figure reproduced with permission from Schneider et al. [57].

The discrepancies in mechanical properties reported for Sylgard 184 are due to several factors. The most noteworthy factor would be the curing temperature, which was found to have a significant influence on its tensile response [58]. The Young's modulus was linearly proportional to the curing temperature while the strain-to-failure decreased accordingly. 
This is likely attributable to enhanced cross-linking between polymer chains at higher temperatures. Another factor that affects the tensile response of polymers is the strain rate upon loading. Since polymers are viscoelastic materials, the strain rate is expected to influence the material response. Schneider et al. [56] studied this effect by measuring the Young's modulus of Sylgard 184 at strain rates ranging from $0.0025-0.1 s^{-1}$ and found an increase of only $2 \%$ over the entire range. The stiffness of the PDMS was not overly sensitive to changes in loading rate, however its influence on tensile strength was not reported.

The shock response of Sylgard 184 was previously characterized through uniaxial plate impact experiments in order to calculate its shock-induced shear strength for impact stresses up to 2.0 GPa [64]. Existing shock velocity data was extracted from literature [65, 66] and combined to obtain representative shock parameters along with Hugoniot stress data. The shock-induced shear strength of Sylgard 184 was found to increase with increasing impact stress and behaved similarly to cross-linked epoxy, although the strength of the latter was much higher. This was attributed to the fact that epoxy was loaded below $T_{g}$, as opposed to the PDMS [64].

\subsection{Mechanical Characterization of PMCs: Previous Work}

It was previously discussed that the general properties of polymers can be improved by chemically bonding the molecular chains (cross-linking) or through physical constraints (e.g., entanglement of long chains) [53]. Integration of filler materials such as fibers or particles has become common practice since it can drastically improve the mechanical properties of a given polymer matrix. A two-phase composite is composed of a certain volume of filler material $v_{f}$ while the remaining volume is occupied by the matrix, i.e., $v_{m}$. The proportion of each phase can be added to obtain the total volume $v_{c}$ of the composite (assuming no voids) [52]. A simple expression that correlates the volume fraction of each component can be obtained by dividing the volumetric additivity relation by the total composite volume $v_{c}$, 
resulting in

$$
\phi_{m}=1-\phi_{f}
$$

where $\phi_{f}=\frac{v_{f}}{v_{c}}$ and $\phi_{m}=\frac{v_{m}}{v_{c}}$ are the volume fractions of the filler and matrix materials, respectively. Similarly, an equation for composite density $\rho_{c}$ can be obtained in terms of filler and matrix proportions by dividing the mass additivity relation by $v_{c}$, which yields

$$
\rho_{c}=\phi_{f} \rho_{f}+\left(1-\phi_{f}\right) \rho_{m}
$$

This demonstrates that composites have some simple additive properties and is known as the rule of mixtures [52]. Prior to discussing the response of particle-filled composites, the relatively simple methods used to predict the mechanical properties of fiber-reinforced PMCs are introduced. Some of these concepts can however be translated to all composite types, e.g., Eq. 2.

\subsubsection{Fiber-reinforced PMCs: Low-Strain-Rate Loading Conditions}

Consider a tensile load $F$ applied on a continuous fiber-reinforced composite in two directions, i.e., parallel to the fiber direction and perpendicular to them. Schematics of these events are shown in Fig. 7. The components are assumed to be perfectly bonded together and so the mechanical coupling between fiber and matrix provides a uniform stress or strain distribution which depends on the fiber orientation relative to the applied force. If the force acts along the fiber direction (Fig. 7a), the two components are described as being coupled in parallel and experience equivalent axial strains $(\epsilon)$, i.e., $\epsilon_{f}=\epsilon_{m}=\epsilon_{c}$. The total stress applied to the composite $\sigma_{c}$ is therefore the weighted sum of the stresses experienced by the fibers $\sigma_{f}$ and the matrix $\sigma_{m}[52]$, as described in

$$
\sigma_{c}=\phi_{f} \sigma_{f}+\left(1-\phi_{f}\right) \sigma_{m}
$$




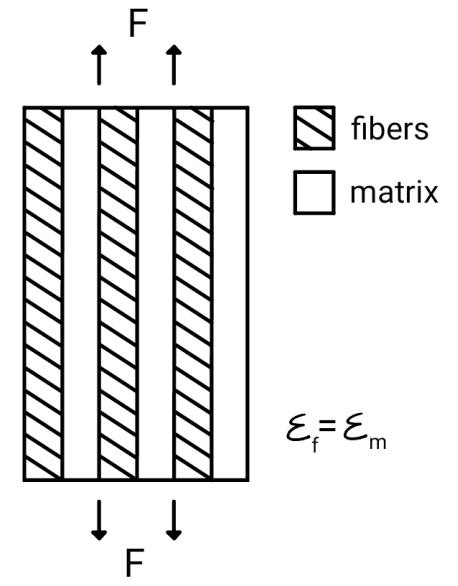

(a)

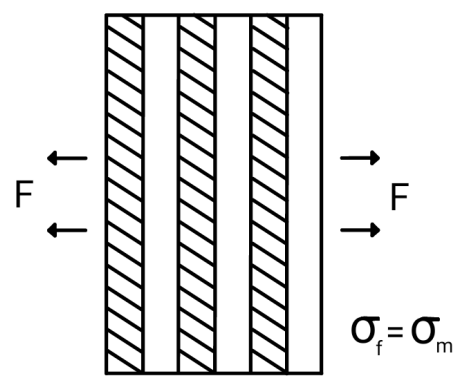

(b)

Figure 7. Schematic of unidirectional continuous fiber-reinforced composite arranged in (a) parallel and (b) series mechanical coupling.

The linear additivity of the tensile stress is another example demonstrating the application of the rule of mixtures. Similarly, the Young's modulus of the composite $E_{c}$ can be obtained by neglecting viscoelastic properties of the polymer matrix and assuming linear elastic behaviour [52]. At high volume fractions, the fibers play a dominant load-bearing role, whereas the polymer matrix serves as a glue to hold the composite together. On the other hand, the polymer matrix plays a much more important role when loaded in a transverse direction relative to the fiber reinforcements, Fig. 7b. In this case, the components are considered to be coupled in series and no longer have equal strains. They are now assumed to experience the same tensile stress as the overall composite, i.e., $\sigma_{f}=\sigma_{m}=\sigma_{c}$. Tensile loads are fully supported by both components and the rule of mixtures can be used to calculate the total strain. The fibers merely serve to reduce the amount of deformable matrix since $E_{f}>E_{m}[52]$. An expression for the composite Young's modulus is obtained for transverse (series) loading scenarios:

$$
E_{c}=\frac{E_{f} E_{m}}{\left(1-\phi_{f}\right) E_{f}+\phi_{f} E_{m}} .
$$

In some circumstances it was found advantageous to integrate discontinuous fibers into polymers instead of long fibers that span the entire material, e.g., manufacturing consid- 
erations. The Young's modulus of such composites often cannot be represented by the theoretical upper and lower bounds (parallel and series arrangement, respectively). Hirsch [67] proposed an intermediate model that lies between the two limits by assigning an $x$ proportion of fillers arranged in parallel configuration and $1-x$ in series [68]. Focus is now shifted to polymers reinforced by discontinuous fillers; theory of short-fiber-reinforced composites will be used to transition into the analysis of particulate composites. Figure 8 illustrates the influence of a perfectly-bonded cylindrical particle or fiber embedded into a softer matrix in terms of the strain field experienced by the system. The stiffer particle resists local deformation, therefore reducing the overall strain of the system. This effect is a direct result from proper load transfer through the filler-matrix interface. The strength of the composite must be related to the strength of these interfacial bonds [52].

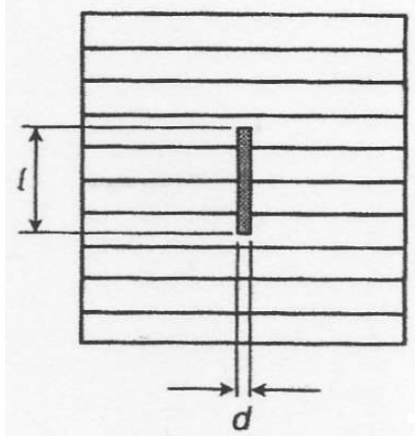

(a)

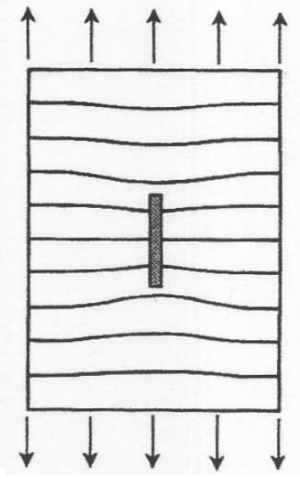

(b)

Figure 8. Effect of particle reinforcement on softer matrix. Horizontal lines illustrate the strain distribution within the material when in its (a) relaxed state and (b) strained state. Figure reproduced with permission from McCrum et al. [52].

The previous analysis assumed that the continuous fibers had no extremities. These areas are however considered to be the weak points in discontinuous fiber-reinforced composites. They do not contribute towards tensile load transfer from matrix to fiber, but rather induce high stress concentrations within the matrix. Maximum interfacial shear stress is located at the fiber ends. As strain is increased, this is where interfacial failure through debonding or shear failure of the matrix begins. The shear strength of an interface depends on three primary factors: the strength of chemical bonds between the fillers and matrix; inter-phase 
friction resulting from pressure applied by the matrix onto the fiber (i.e., thermal expansion coefficient mismatch and solidification shrinkage of polymer); and finally, the shear strength of the polymer matrix [52]. It is the shear stress at the fiber-matrix interface that transmits tension to the fibers. Tensile stresses build up from zero at the fiber ends to a maximum value at its center. A schematic demonstrating the shear and tensile loads experienced by a perfectly-bonded discontinuous fiber is shown in Fig. 9.
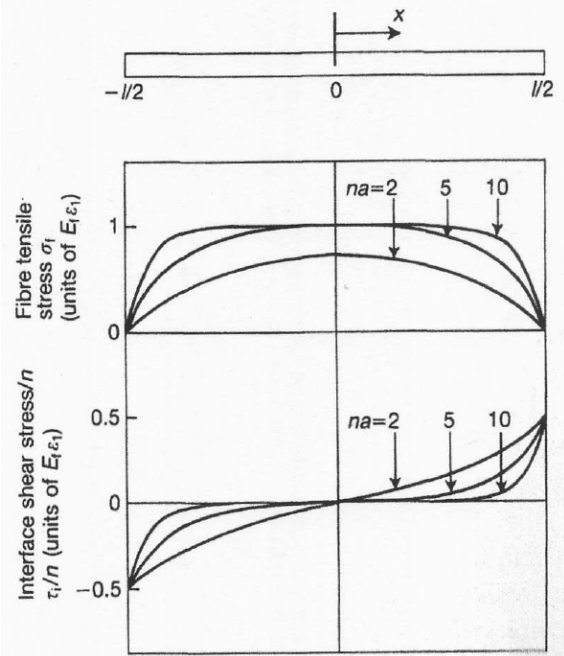

Figure 9. Tensile and shear loads experienced by a perfectly-bonded discontinuous fiber or particulate $\left(a=A R\right.$ and $\left.n \propto \sqrt{G_{m} / E_{f}}\right)$. Figure reproduced with permission from McCrum et al. [52].

The decrease in load-carrying capacity of fiber ends reduces the Young's modulus and tensile strength of the overall composite. Effective load transfer between both phases is partially dependent on the filler aspect ratio $(A R=l / d)$. Figure 9 also suggests that the ratio of matrix shear modulus to filler tensile modulus $\left(G_{m} / E_{f}\right)$ should be as high as possible. Composite strength can still be calculated using Eq. 3, however a mean fiber stress that accounts for fiber ends and debonding must be used instead of $\sigma_{f}$ [52]. Folkes [69] modified the rule of mixtures (Eq. 3) by employing a load transfer efficiency factor $\left(\epsilon_{1}\right)$ and a fiber orientation factor $\left(\epsilon_{0}\right)$ to account for the differences in short fiber-reinforced composites. When fibers are oriented parallel to the tensile load, $\epsilon_{0}=1$, whereas $\epsilon_{0} \approx 0.33$ for randomly dispersed fibers. $\epsilon_{1}$ is more difficult to quantify since it depends on several factors that affect 
the adhesion between both phases, including fiber length (for continuous fibers, $\epsilon_{1}=1$ ) [54]. A critical particle aspect ratio $\left(A R_{c}\right)$ has been derived for discontinuous-fiber composites by equating the interfacial adhesive forces between the two phases with the tensile strength of the filler $[54,69]$. The relation for $A R_{c}$ is shown in

$$
A R_{c}=(l / d)_{c}=\frac{\sigma_{f t s}}{2 \tau_{I}}
$$

where $\sigma_{f t s}$ is the tensile strength of the fiber and $\tau_{I}$ is the interfacial strength between the fiber and matrix. When low- $A R$ fillers are used, an effective coupling agent is quite important in order to diminish $A R_{c}$. Bigg et al. [54] claimed that for randomly dispersed fibers within a matrix, the maximum degree of reinforcement is achieved when the average fiber $A R$ is equal to or larger than approximately ten times the $A R_{c}$ value. They went on to claim that for typical discontinuous fiber-reinforced PMCs, maximum reinforcement is achieved when $A R \geqslant 500: 1$.

The tensile strength of discontinuous fiber-reinforced PMCs is strongly dependent on filler adhesion. On the other hand, the Young's modulus is affected by filler adhesion to a lesser extent. Evidence of this is provided by noting that improperly-bonded fillers still enhance the Young's modulus of a matrix, while decreasing its tensile strength. The modulus is more influenced by filler orientation [54]. A similar modification to the rule of mixtures was suggested to calculate the Young's modulus of short fiber-reinforced composites by incorporating an overall efficiency factor [69].

The degree of filler adhesion depends on the wetting properties of the filler and matrix as well as the amount of surface area available for bonding. The surface area-to-volume ratio $(A / V)$ of a particulate is an important factor to consider and should be as large as possible for improved adhesion. A larger surface area implies that more force is required to reach the shear strength of the filler interface, therefore, delaying debonding. Nano-fillers have gained significant popularity in the recent decades due to their significantly larger $A / V$ 
values relative to micron-sized fillers. Similarly, increasing the $A R$ of a fiber results in larger $A / V$ values, hence their improved adhesion. Decreasing the $A R$ of particulates below unity can also provide a similar enhancement in $A / V$; these types of fillers are called platelets. Figure 10 illustrates the effect of filler $A R$ on its surface area-to-volume ratio [52]. It can be seen that thin platelets and long fibers offer the most interfacial area at a given volume fraction, thus, maximizing particle-matrix interaction.

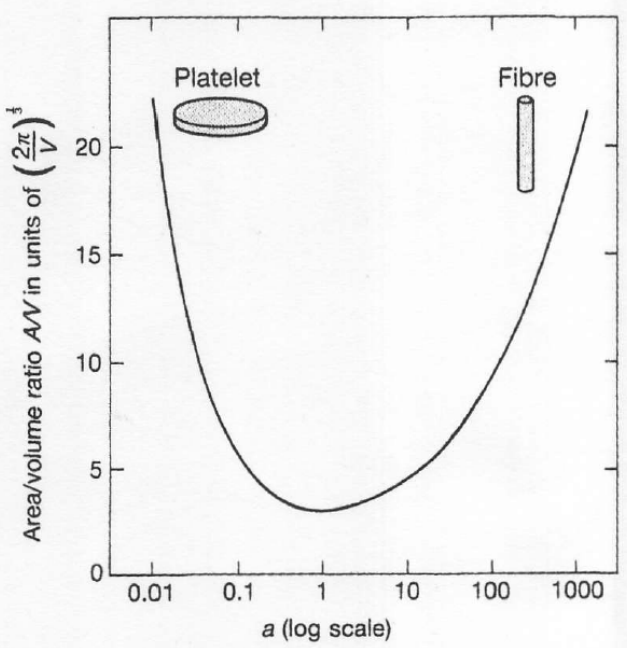

Figure 10. Effect of cylindrical particle aspect ratio $(a=A R)$ on its surface area-to-volume ratio $A / V$. Figure reproduced with permission from McCrum [52].

\subsubsection{Particle-reinforced PMCs: Low-Strain-Rate Loading Conditions}

It is quite common to reinforce polymers with metal or ceramic particles. A variety of particulate shapes are available, e.g., platelets, spherical, irregular/blocky, whiskers, etc. These particle types have been listed in order of increasing $A R$ and provide different degrees of polymer reinforcement. The reinforcement of polymeric materials through particle integration was first observed in carbon black-filled natural rubber [68], which is a popular example of proper composite engineering. The particulates improved the strength, stiffness, and wear resistance of the rubber matrix, therefore enabling its use for car tire applications. Polymer reinforcement resulting from particle integration can be achieved through chemical or physical means [52]. Adsorption of the polymer chains onto the particle surfaces allows for 
efficient load transfer from the matrix to the particles. On the other hand, poorly bonded particles are still able to impose a physical barrier, which hinders polymer chain mobility. The general consensus among researchers is that the addition of rigid particles into a polymer matrix increases the Young's modulus of the system [15]. The degree of improvement could be quite dramatic, as demonstrated by Neitzel et al. [17] who reported an increase in Young's modulus up to $470 \%$ when 25 vol.\% nanodiamond was added to an epoxy matrix.

Ahmed and Jones [68] as well as Fu et al. [15] discuss several theories that attempt to predict the mechanical properties of particulate composites. In particular, many models have been suggested to predict the Young's modulus of particulate composites. An extensive list summarizing the early work on this subject has been provided by Bigg [70]. A note-worthy model for the Young's modulus of elastomeric composites was extracted from Einstein's theory of viscosity for rigid spheres suspended within a liquid. This theory was found to be analogous to the elastic modulus of carbon black-filled rubber having perfect filler adhesion [71] and is described by

$$
E_{c}=E_{m}\left(1+2.5 \phi_{f}\right)
$$

Guth [71] explained that the increase in Young's modulus of filled rubber is due to stress and strain perturbations within the system that are caused by the suspended particles upon deformation, which leads to an increase in elastic energy. Equation 6 indicates a linearly increasing relationship with filler volume fraction. The expression is only valid at low filler concentrations since the theory assumes that the particles act independently, i.e., having no mutual interactions with others nearby [71]. The addition of a non-linear term $\left(14.1 \phi_{f}^{2}\right)$ to Eq. 6 has been suggested for higher filler concentrations to account for the interacting strain fields between neighbouring particles. Particles such as carbon black may form interconnected chains within the matrix at high volume fractions, which could cause an accelerated stiffening effect. A similar expression was derived for rod-like fillers by accounting for particle $A R[71]$.

It is well-known that the addition of rigid particles into a polymer matrix increases the 
stiffness of the system. On the other hand, there is less agreement with respect to the effects of particle integration on the tensile strength of composites since more factors come into play. The most important being the interfacial adhesion strength between matrix and filler, which is less significant for the Young's modulus. Thio et al. [18] demonstrated the importance of proper filler bonding by applying different surface treatments onto glass beads that were used to reinforce polypropylene. Increased adhesive strength was found to improve the tensile strength of the composite.

As previously discussed, polymers reinforced by continuous fibers see an increase in tensile strength since the fibers participate in supporting the load imposed onto the composite. Particulates are typically not able to support the stresses transferred from the polymer due to less efficient inter-phase bonding [70]. The general trend is that composite tensile strength decreases with increasing particle content. The tensile strength of composites $\sigma_{c}$ exhibiting poor filler adhesion has been previously estimated by considering the effective area of continuous matrix that is available to carry the load $[68,72]$. Consequently, $\sigma_{c}$ has been often represented by a power law,

$$
\sigma_{c}=\sigma_{m}\left(1-a \phi_{f}^{n}\right)
$$

where $a$ and $n$ are constants related to stress concentrations and filler geometry, respectively. Nicolais and Narkis [72] suggested a lower bound model that assumes no adhesion between the spherical fillers and the continuous polymer matrix, resulting in $a=1.21$ and $n=2 / 3$. The theoretical model was validated by experiments of glass bead-filled epoxy composites whereas an upper bound for tensile strength was shown to simply equal that of the polymer when perfect adhesion between the phases was maintained [73]. On the other hand, some researchers have suggested that low- $A R$ flakes can provide a partial or limited strengthening effect. This has been represented by a modification of Eq. 7 by introducing a reinforcing factor due to proper adhesion between filler and matrix that competes against the loss of 
strength resulting from stress concentrations at the particle-polymer interface [70]:

$$
\sigma_{c}=\sigma_{m}\left(1-a \phi_{f}^{n}+c \phi_{f}^{d}\right)
$$

The $a$ and $n$ constants are expected to be similar to those resulting from the lower bound equation; therefore, the values for $c$ and $d$ should provide good indication of the degree of particulate adhesion [70]. Bigg demonstrated the use of the upper and lower bound limits for different polymer composites and is shown in Fig. 11.

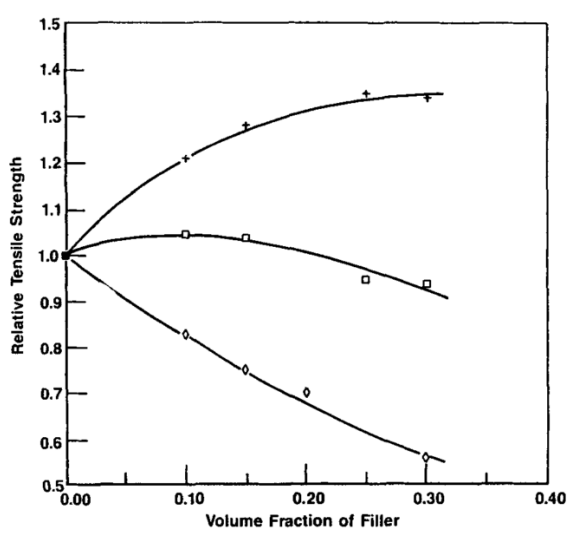

Figure 11. Relative tensile strength $\left(\sigma_{c} / \sigma_{m}\right)$ of various particle-filled PMCs, demonstrating the use of upper and lower bound models: + Noryl-talc, $\square$ ABS-talc, $\diamond$ PP-SiC. Figure reproduced with permission from Bigg [70].

Another relation for the upper limit of tensile strength was suggested by Leidner and Woodhams [74] for polymers filled with spherical particles. Contrary to others, this model requires knowledge of the interfacial tensile strength between both phases $\left(\sigma_{I}\right)$ as well as the shear strength of the polymer $\left(\tau_{m}\right)$, i.e.,

$$
\sigma_{c}=\left(\sigma_{I}+0.83 \tau_{m}\right) \phi_{f}+\sigma_{m}\left(1-\phi_{f}\right)
$$

The expression above depicts a linear relation between composite strength and filler concentration. Many particulate systems have been found to experience partial reinforcement prior to a decline in strength at higher filler concentrations [70]. This cannot be modeled by 
Eq. 9 since it assumes that the upper limit response has no maximum strength value.

Petrovic and Zhang [25] studied the effect of particle addition within two matrices having distinct properties, i.e., a glassy and an elastomeric polyurethane. Significant improvements in the tensile response were obtained for the elastomeric composite while its glassy counterpart saw negligible contributions from the fillers. The elongation to break of the elastomer was claimed to increase by nearly $600 \%$ while the tensile strength increased by over $300 \%$ with the addition of nano-silica. Micro-silica particles showed similar trends, although to a lesser extent. The dramatic improvements claimed in this study seem somewhat questionable due to their magnitude and should require further experimentation for validation.

\subsubsection{Particle-reinforced PMCs: Dynamic and Impact Loading Conditions}

The loading strain rate is evidently another important factor to consider for particle-reinforced PMCs. The trends noted during quasi-static loading do not necessarily translate as the rate is increased. Fracture mechanics of particle-filled PMCs concerns the propagation of cracks that initiate from a local defect, such as a weak particle-matrix interface. Thio et al. [18] concluded that the fracture toughness of glass-reinforced polypropylene increased with weaker particle adhesion at low strain rates due to earlier and more prevalent debonding, which increased the amount of plastic deformation. This effect was however minimized at higher rate (Izod) impact testing. Kushvaha and Tippur [20] found that the fracture toughness

of epoxy increased with glass filler content due to the additional failure modes that arise which further contribute to energy dissipation. A particle morphology study revealed that rod-shaped fillers offered the highest improvement in crack initiation toughness, followed by flake and spherical fillers. The large $A R$ of rods increased the tendency of filler breakage, which was found to be the most effective failure mode. These fillers reduced the crack propagation velocity within the system, although to a lesser extent at higher loading rates. The fracture toughness of neat epoxy and rod-filled composites increased with loading rate; once again, the enhancements were less pronounced at the higher rate. Bigg [70] found that 
the notched Izod impact strength of talc-filled polymers diminished with increasing particle content. The quasi-static tensile strength of the two composites under investigation were represented by the upper bound model, Fig. 11, therefore demonstrating good particle adhesion. Although the composite exhibiting superior filler adhesion performed better under impact, the response was still inferior to that of the unfilled matrix. The author justified the results by stating that cracks can propagate more easily through a polymer-filler interface than through a homogeneous polymer.

There are two fundamental differences that must be recognized when studying the dynamic response of materials, as opposed to quasi-static loading. Firstly, it is important to consider inertial effects within the materials in all governing equations. The second difference arises from the role played by stress waves and the realization that most impact events do not occur under steady-state conditions. It is also important to recognize that the behaviour of each material involved in the impact event influences the overall response [5]. The reader is referred to [75] for more information on shock wave theory.

Setchell and Anderson [22] studied the dynamic response of 43 vol.\% $\mathrm{Al}_{2} \mathrm{O}_{3}$-filled epoxy through planar-impact experiments, which complemented the earlier work from Munson et al. [21] whose volume fractions ranged from $20 \%$ to $43 \%$. The particles consisted of an irregular morphology and ranged from 2 to $30 \mu \mathrm{m}$ in size. The large difference in mechanical properties between both components resulted in a complex behaviour upon shock compression and release. A viscous wave structure was obtained at low shock stresses due to dissipative mechanisms resulting from the microstructure [22]. The authors assumed that the much larger compressibility of the matrix governed the compressive state of the composite. It was suggested that the viscous forces imposed by epoxy hindered relative particle motion within the system during compression, therefore increasing profile rise time. Numerical simulations from Jordan et al. [76] suggested that the shock behaviour of epoxy-based composites (4752 vol. \%) was not strongly influenced by mechanical properties of the aluminum and $\mathrm{Al}_{2} \mathrm{O}_{3}$ particulates, aside from density effects. The epoxy matrix was claimed to drive the behaviour 
of the composite, which is similar to a conclusion extracted from Vogler et al. [23]. It was suggested that the filler volume fraction may not have been high enough for particleparticle interactions to dominate the response [76]. The influence of the polymer matrix is reduced once the particle volume fraction surpasses a certain threshold. Jordan and Spowart [77] observed this behaviour in epoxy- and PMMA-based composites consisting of identical particle compositions. The two unfilled polymers showed distinct peak stresses when subject to dynamic compression using a SHPB. These stresses appeared to converge to a common value as the filler content increased to $50 \mathrm{vol} \%$, thus demonstrating the dominating effect of the particulate phase [77]. Such behaviour was however not observed in the previous numerical simulations at similar volume fractions [76].

The particulate phase is of increasing importance when suspended in a liquid matrix. For this type of system, the fluid primarily serves as a carrier for the solid phase which is dispersed within and held in suspension by electrostatic interparticle forces [39]. A deviatoric stress response was obtained during plate impact experiments on a 48 vol.\% SiC-EG suspension. This behaviour was similar to an elastic solid and demonstrated that the suspension was able to withstand shear stress (see Fig. 12a), therefore, evidence of material strength [46]. It was shown that shock wave loading of dense particle suspensions (DPS) result in an increase in the effective solid phase volume fraction due to the relative compressibility between the liquid and solid components of the system [46]. Consequently, the number of interparticle interactions is expected to increase, resulting in stiffening of the system. Figure 12b shows Hugoniot $\left(U_{s}-u_{p}\right)$ data extracted from the experiments, which was represented by a series of linear fits. A discontinuous rise in pressure occurred above a given impact velocity due to a shock-induced change in microstructure, which was analogous to the non-Newtonian behaviour observed in shear thickening fluids (STFs).

A post-shock solid phase volume fraction was calculated for three mixtures of varying initial particle content and was then correlated to the sudden pressure discontinuities. This transition was found to occur at different critical volume fractions for each mixture, suggest- 


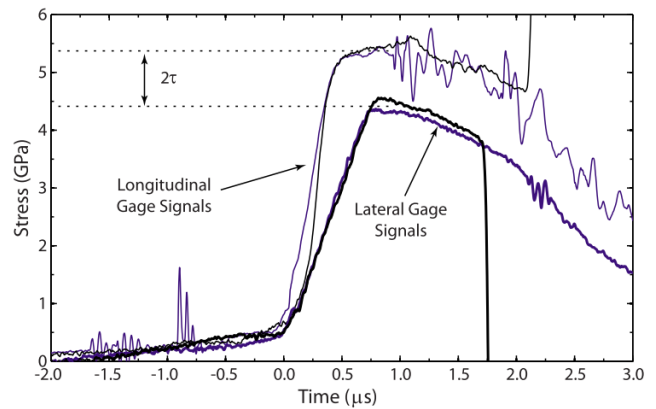

(a)

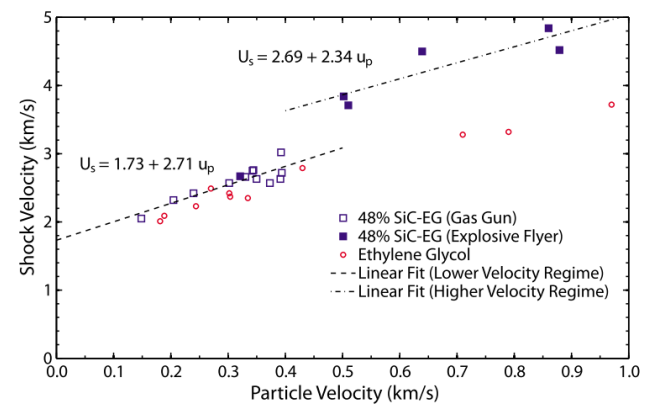

(b)

Figure 12. Experimental data obtained for 48 vol. $\%$ SiC suspension resulting from plate impact experiments showing: (a) deviatoric stress response $\left(V_{i} \approx 1050 \mathrm{~m} / \mathrm{s}\right)$ and (b) $U_{s}-u_{p}$ Hugoniot curve with separate linear fits for the low- and high-velocity regimes. Figure reproduced with permission from Petel and Higgins [46].

ing a complex process dependent on both the initial particle loading fraction and the applied strain rate [46]. The results from Fig. 12 suggest that the particles are dominating the stress state response of the system at high impact velocities through interparticle contacts, which lead to the formation of a network of force chains capable of resisting stress. Results from a 2D numerical model simulation showed that upon shock compression, there were defined structures of particles that carried a higher mean stress than average throughout the domain, thus, confirming the existence of a network of force chains or clusters [39]. A hard-disk model approach was used to simulate the microstructure of a DPS under impact, as seen in Fig. 13 [47]. A partially jammed network (or clusters) of particles was observed and was deemed responsible for the dynamic stiffening of the system. Higher stresses would therefore be required for additional deformation [78].

Marr et al. [79] investigated the shock response of a wetted particle bed through plate impact experiments at varying velocities. It was found that the low strength brittle glass beads began to fracture at pressures as low as $0.5 \mathrm{GPa}$ and were nearly completely pulverized at a shock pressure of $1.5 \mathrm{GPa}$. It should be expected that particle deformation would have an effect on the pressure induced by a shock wave within a dense suspension. Fracture of particles participating in force chains would result in temporary stress relief and in the reorganization of surrounding particles. Such an event would be expected to occur during 


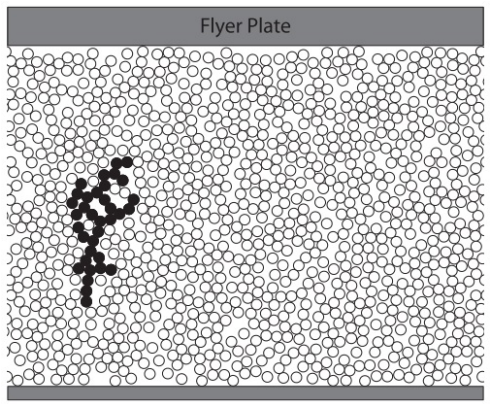

Figure 13. Image resulting from a $2 \mathrm{D}$ hard-disk model of a 41 vol. $\% \mathrm{SiC}$ suspension experiencing a compressive strain of 0.29. The largest force chain, or cluster of particles, is emphasized. Figure reproduced with permission from Petel et al. [47].

ballistic impact. This study clearly illustrates the importance of particle strength when interparticle collisions dominate the material response.

\subsection{Conclusion}

The present chapter discussed basic polymer science along with studies concerning the mechanical characterization of PMCs in order to establish the fundamental knowledge required to analyze the composite systems under investigation. As previously mentioned, our systems consisted of a PDMS elastomer matrix doped with several micron-sized ceramic particles. A description of polymer microstructures was provided in Section 2.1 along with a discussion of the parameters responsible for their varying properties. Cross-linking enabled the synthesis of elastomers such as Sylgard 184, which was used as the matrix material for the present thesis. The viscoelastic tensile response of the elastomer was assessed in Section 2.1.1. A brief discussion of its shock response was also included.

The concept of reinforcing polymers with filler materials was introduced by presenting the rule of mixtures, which enables the calculation of composite bulk properties such as density, Eq. 2. This relation was employed during the synthesis of our materials in order to obtain proper filler volume fractions. Several theoretical models were presented to quantify the Young's modulus and tensile strength of particulate composites $[68,15,70,71,72,73,74]$. In particular, the models investigated the influence of particle volume fraction, aspect ratio, 
and particle-matrix adhesion strength. The fracture toughness of particle-filled polymers was briefly discussed in terms of the parameters stated above as well as strain rate $[18,20,70]$. These parameters will also be considered throughout the present study in order to elucidate the role of particulates within the composite when subjected to quasi-static and ballistic loading.

Section 2.2.3 covered the high strain rate response of particulate composites under impact. Shock compression studies found that the polymer matrix drives the dynamic response of the composite unless the particle volume fraction is sufficiently high $[22,76,23,77]$. Dense particle suspensions experienced a discontinuous jump in shock velocity and pressure when impacted above a certain threshold velocity [46], which was related to microstructural changes within the system causing the formation of temporary force chains $[39,47,78]$. The stiffening phenomenon was dependent on both strain rate and initial volume fraction. Particle breakage was observed when the conditions above were satisfied [79], demonstrating the importance of particle strength. As will be seen in the following chapter, such DPSs experienced a similar dynamic response during ballistic penetration events, therefore providing more evidence of the importance of filler material strength (at high volume fractions only).

Several of the subjects covered herein will be revisited during the presentation and discussion of results. The following chapter discusses the relevant theory of terminal ballistics that is required to properly analyze the penetration response of our particulate composites. 


\section{Terminal Ballistics}

The field of ballistic studies is divided into three general areas: internal ballistics, which focuses on the acceleration of the projectile within the firearm barrel prior to its expulsion; external ballistics, which considers projectile trajectory and aerodynamics during flight; and terminal ballistics, which analyzes the interaction between the projectile and the target during penetration. The latter subject employs several experimental, theoretical, and modeling techniques in order to study and characterize the penetration behaviour of materials.

The dynamic response of a target material transitions through several behavioural stages as the penetration velocity increases. A simplistic material response characterization chart based on incident velocity was introduced by Weirauch [80] and is summarized here. Generally speaking, the target first experiences an elastic response with local plasticity during penetration at low impact velocity. Plastic deformation then begins to dominate at higher velocities and the material experiences a viscous flow while still exhibiting signs of strength. Upon further projectile acceleration, the target demonstrates a fluid-like behaviour and a loss of strength (i.e., hydrodynamic behaviour). At this stage, target density is the dominant parameter dictating projectile deceleration. It should be noted that compressibility effects must also be considered at high impact velocities. The transitions in material response are however very flexible and should only be used as a guide for understanding impact phenomena since there exists a large list of parameters other than impact velocity that are responsible for the deformation of targets [5].

There are many types of projectiles (or threats) that can be considered in ballistics, each of which serve for different purposes. Examples of such penetrators include small arms bullets, long rods, shaped charge jets, and fragment simulating projectiles (FSPs). Investigating the penetration response of FSPs provides us with insight into the behaviour of the material when subjected to ejecta and debris impacts resulting from blasts (e.g., I.E.D. charges). These fragment simulators are typically made of hardened steel and are available in a wide range of masses and nose shapes. Ballistic targets are categorized based on their 
thickness relative to projectile caliber, i.e., thick, intermediate, or thin. Experiments can be classified based on projectile incident (or impact) velocity, $V_{i}$ : low velocity $\left(0<V_{i}<500\right.$ $\mathrm{m} / \mathrm{s})$, ordnance velocity $\left(500 \leq V_{i}<2000 \mathrm{~m} / \mathrm{s}\right)$, and hypervelocity $\left(V_{i} \geq 2000 \mathrm{~m} / \mathrm{s}\right)$ [81]. Nowadays, ballistic impact experiments are often conducted using single-stage or two-stage light gas guns, which are capable of accelerating projectiles well within the hypervelocity range. These function by exposing a diaphragm to a large pressure differential which results in its rupture followed by the sudden expansion of a highly compressed gas (e.g., helium or hydrogen) within a chamber behind the projectile. The present thesis investigates the penetration response of thick composite targets by 16-grain FSPs that were accelerated by a single-stage gas gun within the low and ordnance velocity range.

Terminal ballistics has been thoroughly studied by numerous researchers and spans a wide range of subjects. Extensive theoretical work has been dedicated to the penetration of metallic plates, which has produced a large amount of experimental data. The basic principles which pertain include the conservation laws, role of wave propagation, influence of inertia, and an understanding of the dynamic behaviour of materials [5]. An overview of some studies is provided in this chapter while only focusing on topics relevant to the present thesis. For more information, the interested reader is referred to extensive reviews on the subject $[81,82,83]$. It is important to have a basic fundamental understanding of the behaviour of materials during ballistic (high strain rate) penetration. The present chapter shall discuss various methods to assess their performance and correlate their response to failure mechanisms through the use of simple analytical models. Examples of such analysis using residual (or exit) velocity data are provided and will be subsequently employed to characterize the response of our particle-filled elastomeric systems. 


\subsection{Penetration of Rigid Projectiles in Semi-infinite Metallic Tar- gets}

Acquiring knowledge of the mechanics of deep penetration is the preliminary step towards studying terminal ballistics since these tests reveal certain fundamentals of the penetration behaviour of materials and therefore has a tutorial role. There is an extensive database of studies spanning over the century that report the depth of penetration of several projectiles within various semi-infinite targets, e.g., $[84,3,85]$ A semi-infinite target is one that is large enough to ensure that the lateral and distal faces do not influence the penetration process, resulting in a more simplistic analysis. The penetration of a rigid projectile is a physical process that is governed by the resisting force imparted by the target which acts to decelerate the penetrator [81]. Several empirical and analytical models have been developed to predict the depth of penetration within a target $(P)$. These are derived by integrating Newton's second law of motion. The underlying requirement to obtain a solution is to develop an expression that accurately describes the deceleration process of the projectile $(a=a(V))$. Several authors have suggested functional forms for $a(V)$ throughout the years. These are all special cases of the general expression [81]:

$$
a(V)=C+A V+B V^{2},
$$

where $A, B$, and $C$ are constants which must be determined empirically through experimentation and $V$ is the projectile velocity. The most popular forms of Eq. 10 are: Euler-Robins $(a=C=$ constant $)$, Poncelet $\left(a=C+B V^{2}\right)$, and Resal $\left(a=A V+B V^{2}\right)[81]$.

Through experimentation, it was found that the deep penetration of rigid rods in semiinfinite metallic targets can be characterized by a constant deceleration (Euler-Robins) [81, 84] and is described by

$$
P=\frac{V_{i}^{2}}{2 a}
$$


where $V_{i}$ is the incident velocity of the projectile. This model has been validated by Rosenberg et al. [86, 81] through the interpretation of results obtained from Piekutowski et al. [84] for an ogive-nosed projectile impacting slabs of aluminum, as seen in Fig. 14 [81]. The model correlated well with the experimental data at high velocities, thus, validating the previous statement.

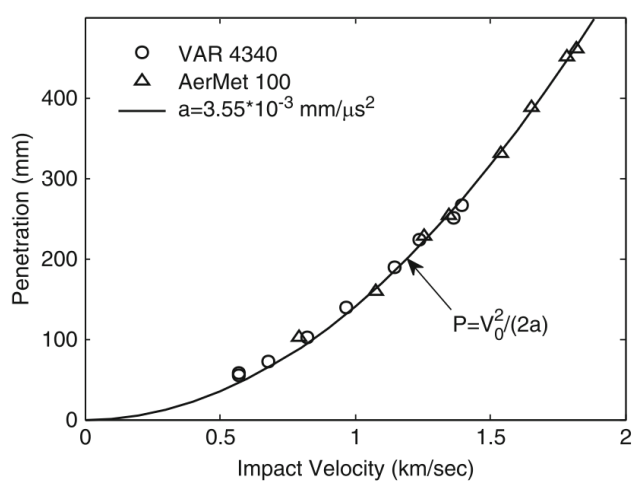

Figure 14. Depth of penetration data obtained from a semi-infinite aluminum slab impacted by a rigid ogive-nosed projectile, demonstrating validation of the constant deceleration theory. Figure reproduced with permission from Rosenberg and Dekel [81].

Rosenberg and Dekel [86] performed numerical simulations to verify the effect of several target-projectile parameters. The constant deceleration assumption has been further validated by simulating the deceleration of an ogive projectile penetrating a semi-infinite aluminum slab at velocities ranging from 0.5 to $1.5 \mathrm{~km} / \mathrm{s}$, Fig. 15a [86]. The deceleration value remained unaffected when varying the impact velocity. Based on the second law of motion (Newton), it can be stated that a constant deceleration process corresponds to a constant force exerted by the target. An expression for the constant deceleration of a projectile has been derived using this notion:

$$
a_{a v}=\frac{A Y}{\rho_{p} L_{e f f}}
$$

where $\rho_{p}$ and $L_{\text {eff }}$ are the density and effective length of the projectile, respectively, $Y$ is the yield strength of the target, and $A$ is a factor that must be determined through a cavity expansion model [86]. 


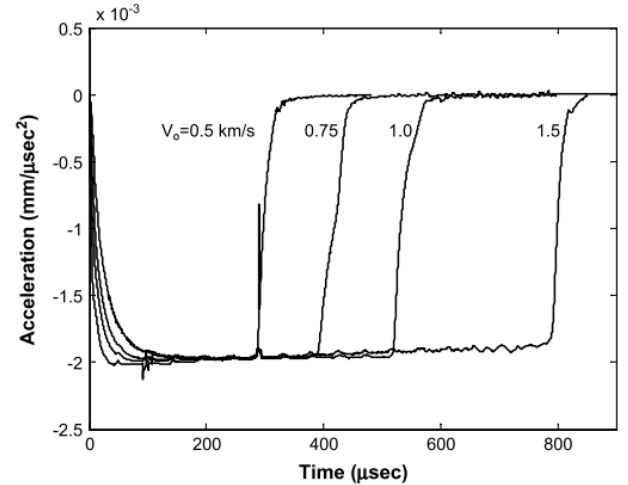

(a)

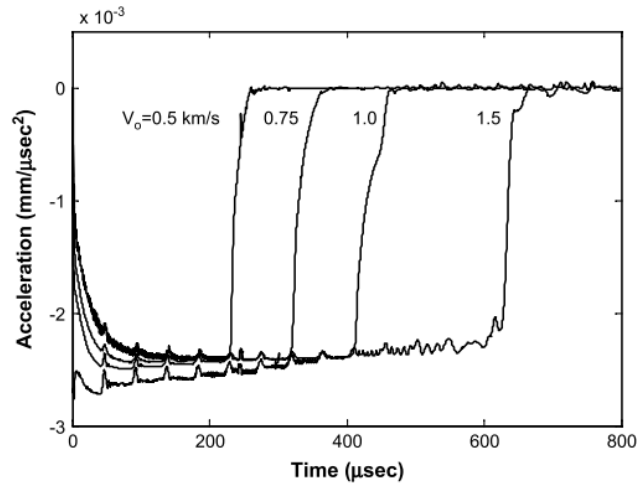

(b)

Figure 15. Numerical simulation demonstrating: (a) constant deceleration of ogive projectile, (b) velocity-dependent deceleration of hemisphere projectile. Figures reproduced with permission from Rosenberg and Dekel [86].

A slightly different response was however observed for simulations concerning a hemispherical projectile nose. Figure $15 \mathrm{~b}$ demonstrates that a pronounced change in deceleration $(\approx 14 \%)$ occurred throughout the penetration event as the impact velocity increased to $1.5 \mathrm{~km} / \mathrm{s}$. This is evidence that not all deep penetration can be described by a constant deceleration. The result suggests that the nose shape plays an important role in the penetration process since the deceleration of ogive projectiles within an identical target remained relatively constant [86].

The fact that the deceleration of a hemispherical projectile begins to vary as the velocity increases suggests that there exists a threshold velocity that is dependent on projectile nose shape above which an inertia term that contributes to additional stress must be included [86]. This inertial effect was found to be also dependent on the target material, as illustrated in Fig. 16 which depicts the deceleration history of projectiles penetrating aluminum and steel slabs. A significant variation in deceleration was noted for the steel targets, which was expected to be a consequence of its much higher density (i.e., higher inertia) [86].

It should be noted that all simulations discussed above were assigned a elastic-perfectly plastic material response, which does not consider strain-hardening and strain-rate sensitivity. So far, we discussed the applicability of the constant deceleration assumption and 


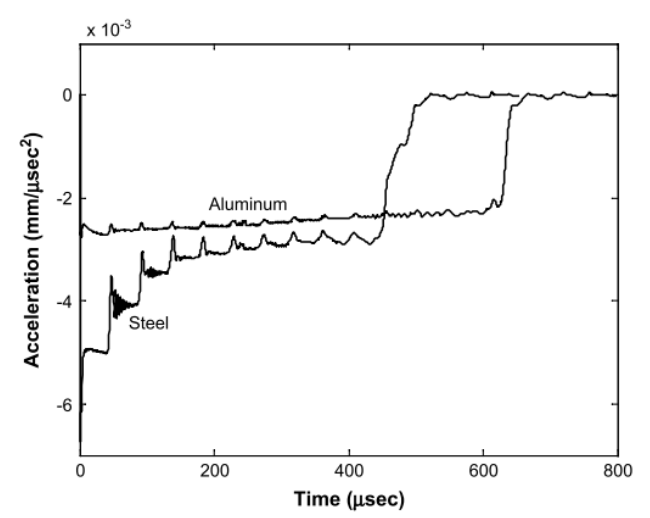

(a)

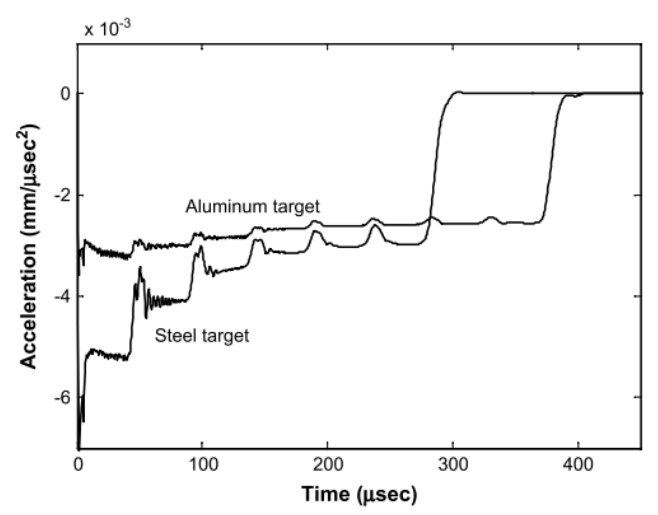

(b)

Figure 16. Numerical simulation showing the effects of target inertia on the deceleration process of (a) hemispherical $\left(V_{i}=1.5 \mathrm{~km} / \mathrm{s}\right)$ and $(\mathrm{b})$ blunt-nosed projectiles $\left(V_{i}=1.0\right.$ $\mathrm{km} / \mathrm{s}$ ). Yield strength of all materials set to $0.4 \mathrm{GPa}$. Figures reproduced with permission from Rosenberg and Dekel [86].

noted that there is a threshold velocity above which inertial effects must be considered. The threshold was found to be dependent on the combination of projectile nose shape and target material properties. Numerical simulations have suggested that there exists such a threshold velocity for every projectile-target combination [86]. This behaviour has been correlated to the cavitation phenomenon described by Hill [87].

\subsubsection{Cavitation Phenomenon}

During the Second World War, Hill [87] investigated the penetration resistance of very thick armour and found that the penetration channels were slightly bell-shaped; although the projectile cores did not deform within the range of velocities considered $(1.0-1.5 \mathrm{~km} / \mathrm{s})$, the entry hole had a slightly bigger diameter than the projectile itself. Hill called this phenomenon cavitation, as per the term employed in hydrodynamics and attributed this effect to a combination of projectile velocity, nose shape, and target properties such as density and strength. A schematic of this phenomenon is illustrated below. The author postulated that this phenomenon had an adverse effect on the performance of anti-tank weapons because of the additional kinetic energy that was invested to expand the cavity diameter through plastic deformation [87]. When cavitation is considered, the retarding force exerted by the 


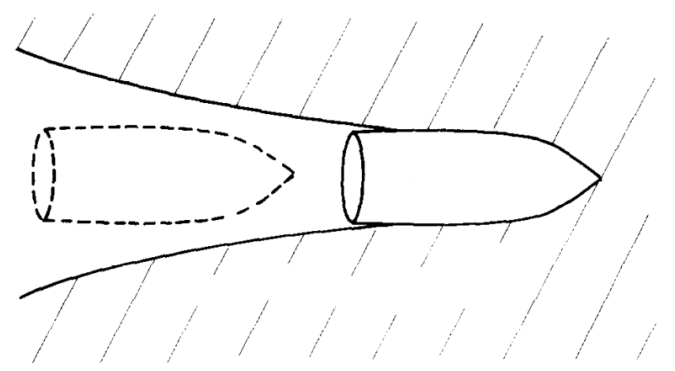

Figure 17. Schematic of cavitation phenomenon during the initial penetration stage. Figure reproduced with permission from Hill [87].

target can be expressed as the sum of two terms. The first term is related to the quasi-static stress exerted by the target material while the second is a dynamic Bernoulli-type term that deals with its inertia [86]:

$$
F=M \frac{d V}{d t}=\frac{\pi D^{2}}{4}\left(A Y+b \rho_{t} V_{w}^{2}\right)
$$

where $b$ and $A$ are determined through the cavity expansion model, $\rho_{t}$ is the target density, $D$ is the projectile diameter, and $V_{w}$ is the radial velocity of the cavity wall [88]. For impact velocities below the critical velocity for cavitation $\left(V_{i}<V_{\text {cav }}\right)$, the projectile nose experiences a relatively constant resisting force and is expected to remain in full contact with the target interface during the penetration process. At higher velocities $\left(V_{i}>V_{\text {cav }}\right)$, the force becomes velocity-dependent due to this inertial cavity expansion phenomenon, resulting in the separation of a portion of the nose and the target at their interface $[86,87]$.

Rosenberg and Dekel [86] performed 2D simulations of rigid rods penetrating steel and aluminum slabs of equivalent strength to further investigate the cavitation phenomenon and concluded that there exists a threshold velocity, $V_{\text {cav }}$, for every rod-target pair. The cavitation velocities were calculated with the help of their simulation results and the expression

$$
V_{c a v}^{2}=\frac{R_{t}}{B \rho_{t}}
$$

where $R_{t}$ is the resisting stress of the target, $\rho_{t}$ is the target density, and $B$ is the nose shape factor [86]. The values obtained from this process are shown in Table 1 below. The 
nose shape factor $B$ was first calculated based on the estimated $V_{\text {cav }}$ range and the predetermined $R_{t}$ values, which then led to the calculated $V_{c a v}$ values. The $B$ values were very similar for both steel and aluminum targets, demonstrating that it is a purely geometric factor [86]. Another conclusion to note is that $B$ varied quite significantly between different nose shapes, implying that projectile nose shape has a quite large influence on cavitation.

Table 1. Calculated $V_{\text {cav }}$ values $(\mathrm{km} / \mathrm{s})$ for several rod-target combinations. Table reproduced with permission from Rosenberg and Dekel [86].

\begin{tabular}{lccc}
\hline Nose Shape & $B$ & Aluminum $(0.4 \mathrm{GPa})$ & Steel $(0.4 \mathrm{GPa})$ \\
\hline Ogive & 0.15 & 2.15 & 1.385 \\
Conical & 0.24 & 1.73 & 1.115 \\
Hemisphere & 0.50 & 1.30 & 0.815 \\
Blunt & 1.25 & 0.85 & 0.525 \\
\hline
\end{tabular}

The effect of cavitation on the deceleration history of a projectile can be observed in Fig. 15b and 16. Qualitatively, cavitation can be described by a steep initial rise in projectile deceleration followed by a moderate decrease until a constant value is reached. Upon examination, it was deduced that the initially enlarged penetration channel became narrower as the rod slowed down. Once the threshold velocity $V_{\text {cav }}$ was reached, a constant diameter equal to that of the projectile was maintained and the constant deceleration assumption was revisited for the remaining penetration depth [81].

The physical process involved in the cavitation phenomenon is quite logical. While the projectile velocity is below the cavitation threshold, the strength of the target material restricts its lateral motion upon penetration, resulting in a cavity diameter equal to that of the projectile. As the velocity increases above this threshold value, the lateral momentum imparted by the projectile is sufficiently high to initiate cavitation, i.e., lateral expansion. The projectile must invest extra work in plastically deforming the cavity to a larger diameter in addition to the energy required for its axial penetration process [81]. The assumption that the penetration process of projectiles occurs at a constant deceleration should therefore only be incorporated when one is certain that the cavitation phenomenon is not present during target penetration. 


\subsubsection{Entrance Phase Effect}

Projectiles also decelerate at a varying rate during the initial penetration stage of the target, i.e., at the entry point. The projectile experiences a lower deceleration than at deep penetration because the material in front of the nose is capable of flowing backwards, towards the free surface nearby. This is based on the concept that the target material is encouraged to flow in the path of least resistance. The resulting retarding force exerted on the projectile is therefore lower than that at deep penetration. This phenomenon is termed as the entrance phase effect and is responsible for penetration depths that are relatively larger than anticipated. This effect is more notable at low impact velocity, as seen in Fig. 14 [81]. Once the projectile surpasses the entrance phase and has reached deep penetration, the retarding force is increased since the material in front of the projectile nose hast lost its escape route. Target material must then be pushed axially along with the projectile or move laterally away from the penetration trajectory [81]. At higher impact velocities, the influence of the entrance phase is negligible relative to the entire penetration process and so the constant deceleration model converges with the data points. The entrance phase region can be better conceptualized by observing the simulated deceleration history of a rod as shown in Fig. 18. Other examples of this effect are demonstrated in Fig. 15, which also shows a diminishing influence as the impact velocity increases. The duration within the entrance phase shortens because it takes less time for the projectile to reach the specified depth for deep penetration [81].

The entrance phase length is strongly dependent on the projectile nose shape, suggesting that this phenomenon is more dominant for some projectiles as opposed to others. As seen in Fig. 18, the entrance phase can be separated into two regimes: the first portion consists of a steep deceleration profile due to the projectile nose fully embedding itself within the target; the second portion is described by a more gradual increase in deceleration resulting from the

backflow of material towards the free surface [81]. Rosenberg and Dekel [86] estimated (using numerical simulations) that the entrance phase length for ogive-nosed projectiles spans ap- 


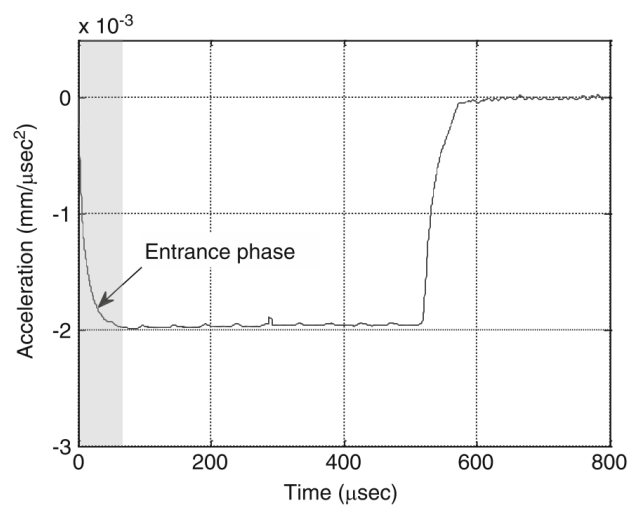

Figure 18. Deceleration history of an ogive-nosed rod penetrating an aluminum slab at 1.0 $\mathrm{km} / \mathrm{s}$. Figure reproduced with permission from Rosenberg and Dekel [81].

proximately four to six projectile diameters (4-6D). Other simulations from the same group have demonstrated that blunt-nosed projectiles (e.g., hemispherical or flat-nose) experience shorter entrance phase times because of their smaller nose length which results in shorter embedment times. The entrance phase length for hemispherical projectiles was estimated to be $3 D$ [89], which is approximately half the length of the ogive-nosed projectile. One could expect a similar entrance phase length for flat-nosed projectiles. These depths describe the dominant range where the projectile deceleration is not constant; however, care should still be taken when incorporating the constant deceleration model for penetration depths passed the dominance range. The depth at which the constant deceleration model becomes valid is called the influence range [81]. Passed this point, the portion of the penetration history that falls below the average deceleration is negligible relative to the entire process and so Eq. 11 can be used to accurately predict penetration depth. It should be noted that as the impact velocity exceeds the cavitation threshold, the entrance phase effect gets negated by the inertia contribution from cavitation. This can be visualized from Fig. 15b, which shows the disappearance of the entrance phase once the impact velocity increased to $1.5 \mathrm{~km} / \mathrm{s}$.

The effect of the entrance phase becomes much more dominant for short projectiles. Their entire penetration process only reaches a couple diameters in depth, therefore remaining within the influence range. Figure 19 shows the deceleration history of short ogive-nosed projectiles, which clearly demonstrates a varying deceleration throughout the entire pene- 
tration process for impact velocities below $1.5 \mathrm{~km} / \mathrm{s}[89]$.

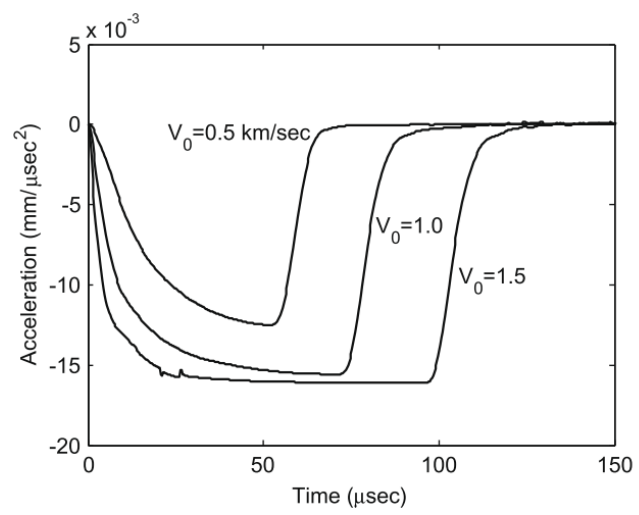

Figure 19. Deceleration history of short ogive-nosed projectiles penetrating aluminum slabs. Figure reproduced with permission from Rosenberg and Dekel [89].

\subsubsection{Role of Projectile and Target Properties on Deep Penetration}

Several types of projectiles have been used to counter ballistic armours; each of these being optimized for a specific purpose. It is crucial that the armour designer be aware of the effect that certain projectile properties have on the penetration response of a target.

The deceleration process of a rigid projectile occurs through a series of elastic waves that continuously travel along the length of the rod and reflect back as release waves upon reaching a free surface. This suggests that a change in velocity initiating at the projectile head takes a certain amount of time to reach its tail, resulting in a periodic time shift between the deceleration of each extremity $[86,81]$. The constant time shift that results in the alternating (out-of-phase) deceleration of each projectile extremity is equal to the time required for the elastic wave to reach the opposite end, i.e., the projectile length divided by the elastic wave velocity of the material: $C_{b}=(E / \rho)^{0.5}$, where $E$ is the Young's modulus of the rod and $\rho$ is its density [81]. It should be evident that the projectile length plays an important role for its step-wise deceleration and overall penetration process. Rosenberg and Dekel [86] simulated the average deceleration of two ogive projectiles and determined that the deceleration was inversely proportional to their length. Since quicker cycles of reverberating waves cause for higher deceleration values, it is evident that shorter projectiles 
do not penetrate as deep and should not be characterized by a constant deceleration value. This latter statement is supported by the deceleration histories in Fig. 19.

Another factor that influences the deceleration process is the choice of projectile material. To prove this, we shall first discuss the stresses of the waves generated from the impact. The interested reader is referred to Ref. [75] for additional information on shock physics. The well-known relation between stress $\sigma$ and the particle velocity $v$ in a long elastic bar is $\sigma=$ $\rho C_{b} v$, where $\rho$ and $C_{b}$ are the density and elastic wave velocity of the material, respectively [81]. This expression can be re-written in terms of the velocity steps $\Delta V$ experienced by the projectile. The amplitude of these steps are equal to twice the particle velocity behind the reverberating waves $(\Delta V=2 v)$ since the particle velocity is doubled with each reflection from the projectile extremity [81]. The modified version of the stress wave relation is shown below

$$
\sigma=\rho C_{b} \Delta V / 2
$$

For a non-cavitating penetration event, the resisting stress exerted by a target is equal to the amplitude of the stress waves generated in the projectile upon penetration and is dependent on the material strength as well as the projectile nose shape [86, 81]. According to Eq. 15 and the previous statement, the velocity steps are only dependent on the product of the stress wave velocity and the projectile density [81]. To validate these claims, Rosenberg and Dekel [86] performed numerical simulations to compare the penetration of aluminum targets using steel and aluminum rods. The results showed a much higher average deceleration value for aluminum projectiles, corresponding to their larger velocity decrement steps. Since steel and aluminum have relatively similar wave velocities, Eq. 15 implies that the differences in velocity steps result from the density mismatch between the projectiles. In fact, the density ratio between steel and aluminum is equal to approximately three, which aligned very well with the observed differences in velocity steps [86, 81].

The effect of projectile nose shape has long been a subject of interest for several re- 
searchers $[86,90,91,92,93,94,95]$ and should be revisited. We have already discussed the effects of nose shape in terms of the cavitation and entrance phase phenomenons and so focus will now be put on its general influence on the deceleration process. It was briefly mentioned that the resisting stress exerted by the target, which is equal to the stress wave amplitude generated in both materials, is also dependent on the projectile nose shape. That being said, the projectile deceleration (related to the velocity decrement steps, $\Delta V$ ) should be strongly influenced by nose shape selection through Eq. 15. The simulation results in Fig.

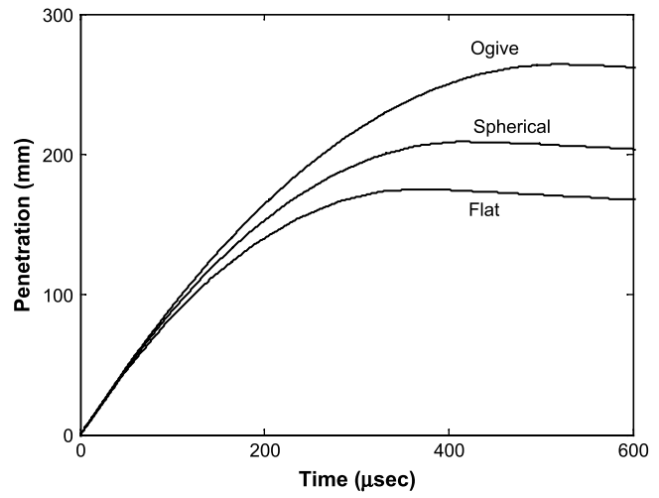

(a)

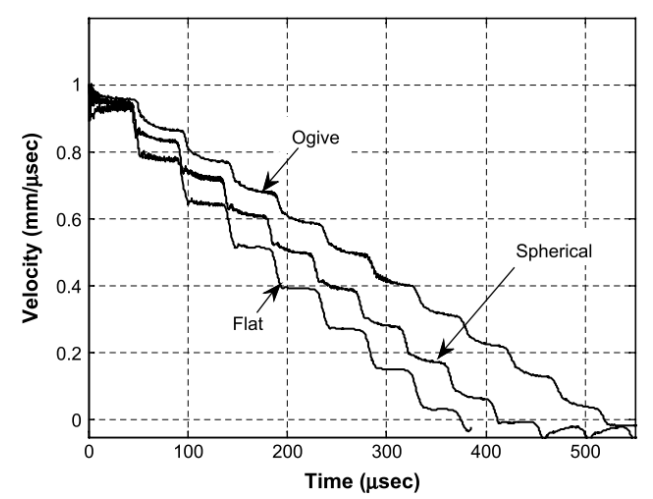

(b)

Figure 20. Numerical simulations of penetration depth results of rigid rods with various nose shapes into semi-infinite aluminum targets. Figures reproduced with permission from Rosenberg and Dekel [86].

20a show that ogive-nose projectiles achieve the highest penetration depth in a semi-infinite aluminum slab, followed by hemispherical- and flat-nosed projeciles, respectively [86]. The varying amplitudes of the velocity steps seen in Fig. 20b together with the stress wave relation (Eq. 15) allow us to conclude that ogive-nosed projectiles experience the least resisting stress while flat-nosed projectiles generated the highest stress magnitudes. It should however be kept in mind that the simulations assumed a metallic target having an elastic-perfectly plastic behaviour (von-Mises yield criterion), which ignores strain-rate and strain-hardening effects [86]. 


\subsection{Analytical Penetration Models of Finite-thickness Metallic Targets}

Studying the penetration behaviour of semi-infinite targets has revealed several fundamental phenomenons relevant to penetration mechanics. The analysis of finite-thickness targets is much more practical in terms of the development of armour systems. A different approach is adopted here, as compared to the previous analysis, since residual velocity data is gathered as opposed to penetration depth results.

The first observation that should be acknowledged when studying the penetration of finite-thickness targets is that the plate thickness required to stop a projectile will always be larger than the penetration depth of the same projectile into a semi-infinite target. This is due to the back-face (or distal) surface which lowers the resisting force exerted on the projectile [81], similar to the entrance phase effect. The immediate consequence of this back-surface effect is that a constant deceleration process cannot be assumed for thin and intermediate thickness plates since the distal surface exerts its influence during or shortly after the entrance phase. This is demonstrated in Fig. 21a, which shows the simulated deceleration history of a projectile impacting a semi-infinite and finite-thickness aluminum target [89]. One can notice a very brief region of constant deceleration around 100 microseconds prior to the distal surface dominating the remainder of the response.

Figure $21 \mathrm{~b}$ illustrates the effect of the distal surface on targets of varying thicknesses. It can be noted that the peak deceleration value gradually increases with thickness, although the impact velocity remained constant. This is a direct result of the back-face surface imposing its lower resisting force while still within the dominant range of the entrance phase, hence, preventing the target to reach its maximum retarding force. Once the target thickness

increased to $100 \mathrm{~mm}$, a constant deceleration (and resisting stress) was momentarily achieved with a maximum value more or less in line with that of the semi-infinite target.

The interaction between the back-surface of the target and the projectile can induce several failure mechanisms during penetration, consequently affecting the retarding force. 


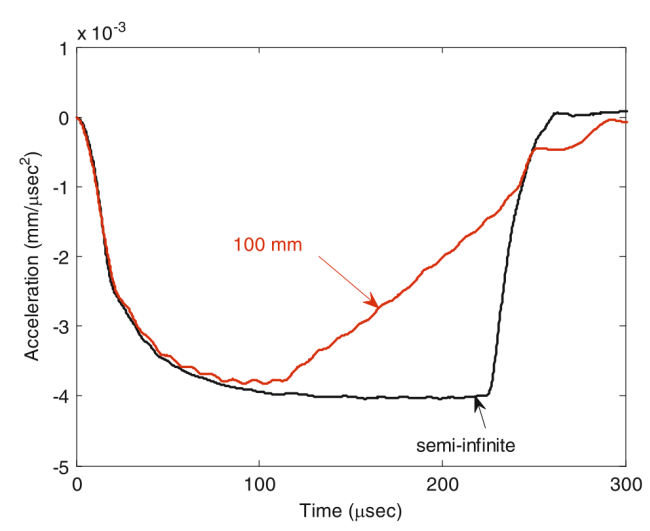

(a)

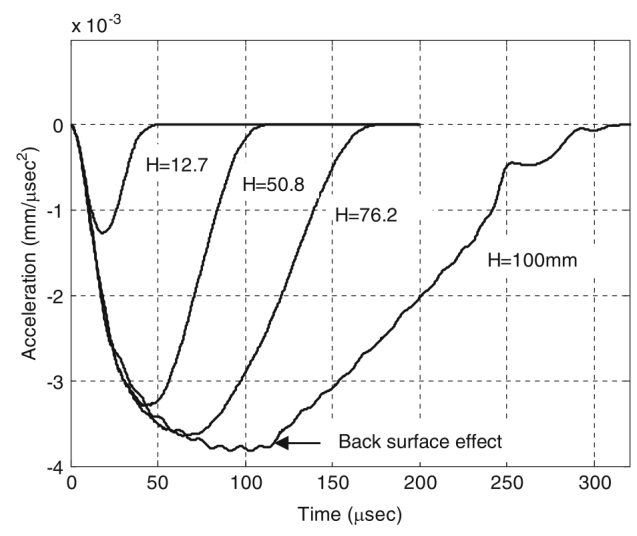

(b)

Figure 21. Numerical simulation of deceleration history of a conical-nosed tungsten projectile penetrating $\left(V_{i}=800 \mathrm{~m} / \mathrm{s}\right)$ : (a) semi-infinite and finite-thickness aluminum targets and (b) targets of varying thickness. Figures reproduced with permission from Rosenberg and Dekel [81].

Figure 22 illustrates some of the failure modes that can occur during the penetration of a finite plate [81]. Wingrove [96] studied the failure mechanisms involved during the penetration of a 10 mm-thick aluminum alloy by ogive, hemispherical, and blunt projectiles (see Fig. 23). These images demonstrate the effect that the projectile nose shape has on the failure mechanisms. The sharp-nosed projectile induced a discing (or scabbing) failure mechanism at the back face (Fig. 23a) while the blunt projectiles accelerated a plug ahead of their noses. Such failure mechanisms will be discussed in more detail later on.

\subsubsection{Penetration of Metals by Sharp Projectiles}

The penetration of ductile metallic plates by sharp-nosed projectiles occurs through the lateral displacement and compression of target material ahead of the projectile nose [97]. This type of penetration event, which will be referred to as perforation, involves the ductile hole expansion process (DHE). An example of this event is shown in Fig. 24 [98].

A relatively simple analytical model was developed by Recht and Ipson [97] that predicts the residual velocity of conical and ogive-nosed projectiles perforating ductile metallic plates. The event can be described by employing the conservation of energy law as follows: 


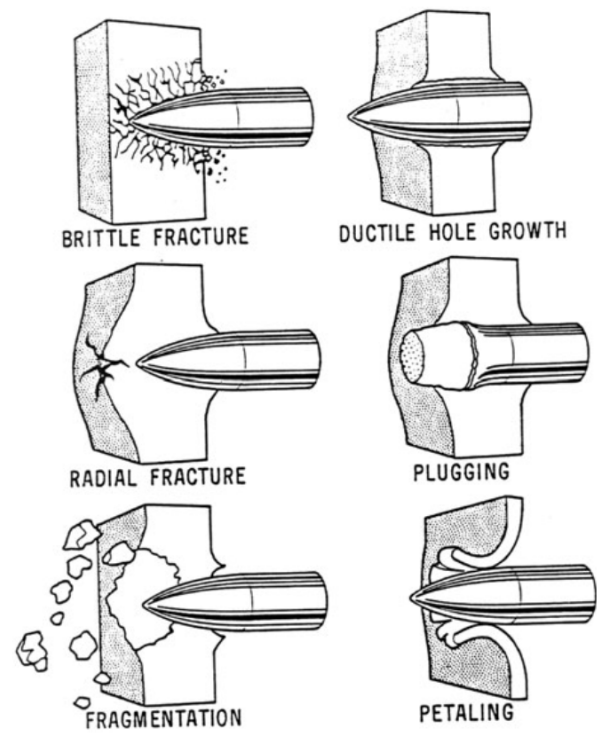

Figure 22. A variety of failure mechanisms found in finite targets resulting from different impact events. Figure taken from Rosenberg and Dekel [81].

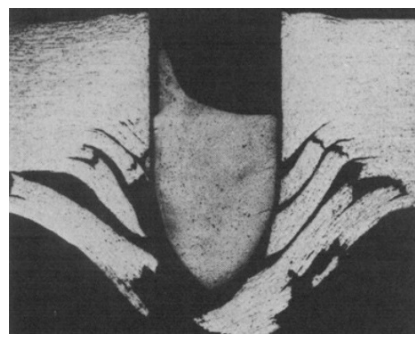

(a)

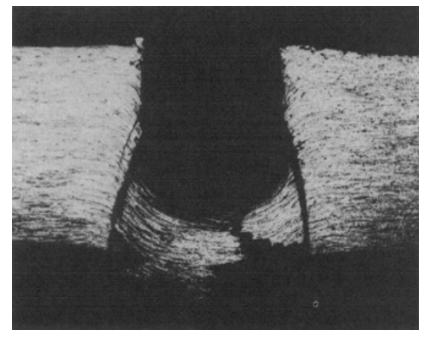

(b)

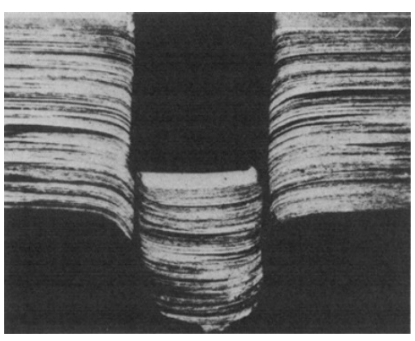

(c)

Figure 23. Failure mechanisms in a $10 \mathrm{~mm}$ thick 2014-T6 aluminum alloy penetrated by (a) ogive, (b) hemispherical, and (c) blunt projectiles. Figures reproduced with permission from Wingrove [96].

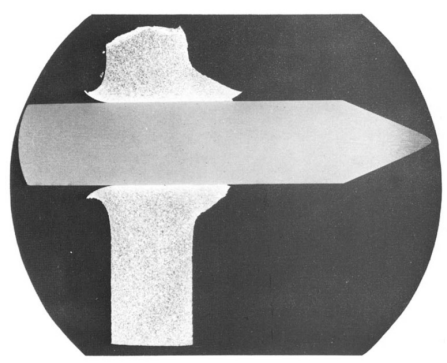

Figure 24. Example of ductile hole expansion resulting from conical projectile perforating a mild steel plate. Figure reproduced with permission from Woodward [98]. 


$$
\frac{1}{2} M_{p} V_{i}^{2}=\frac{1}{2} M_{p} V_{r}^{2}+W_{p}
$$

where $M_{p}$ is the projectile mass, $V_{i}$ is the incident (or impact) velocity, and $V_{r}$ is the residual (or exit) velocity. The $W_{p}$ term corresponds to the work done by the projectile to displace and compress the material ahead of it during penetration. The authors claim that $W_{p}$ is related to the dynamic shear strength of the metal and that this strength is not strongly influenced by velocity [97]. Assuming a constant work contribution during perforation, this value can be quantified by imposing a limiting condition: if $V_{r}=0, V_{i}=V_{b l}$, thus,

$$
W_{p}=\frac{1}{2} M_{p} V_{b l}^{2}
$$

where $V_{b l}$ is the ballistic limit velocity for a given target-projectile combination. The work term (Eq. 17) can then be substituted into Eq. 16 in order to obtain an expression for the residual velocity. The result is often normalized with respect to $V_{b l}$ since this allows for perforation data to collapse onto a single curve [97]. The expression for $V_{r} / V_{b l}$ is shown below and shall be referred to as the RI perforation model:

$$
\frac{V_{r}}{V_{b l}}=\sqrt{\left(\frac{V_{i}}{V_{b l}}\right)^{2}-1}
$$

The equation above accounts for target density, strength, and projectile characteristics through the ballistic limit velocity. The analysis did not however consider inertial effects that arise from cavitation, i.e., energy invested in lateral expansion of the target. This signifies that the model could only be accurate for impact velocities below the cavitation threshold.

The failure mode resulting from penetration and consequently, the resisting stress exerted by a plate is strongly dependent on the $H / D$ ratio, i.e., the plate thickness $H$ relative to the projectile diameter $D$. Rosenberg and Dekel [89] introduced a term called the effective resisting stress, $\sigma_{r}$, in order to avoid dealing with complex time-varying stresses induced by the free surfaces of the target. The idea is that one can assign a constant stress value that 
results in the same change in projectile velocity to describe a penetration event. An equation for this was derived using the equation of motion (Newton), resulting in

$$
\sigma_{r}=\frac{\rho_{p} L_{e f f} V_{b l}^{2}}{2 H}
$$

where $\rho_{p}$ and $L_{e f f}$ are the density and effective length of the projectile, respectively.

Rosenberg and Dekel [99] attempted to correlate the effective stress normalized by the flow stress of the target $\sigma_{r} / Y_{t}$ to $H / D$. Numerical simulations of various combinations of rigid sharp-nosed projectiles and targets were performed while ignoring strain hardening and rate effects in order to focus on the influence of target strength. Their simulation results were compiled into two plots, which were separated at $H / D=1$, as seen in Fig. 25 .

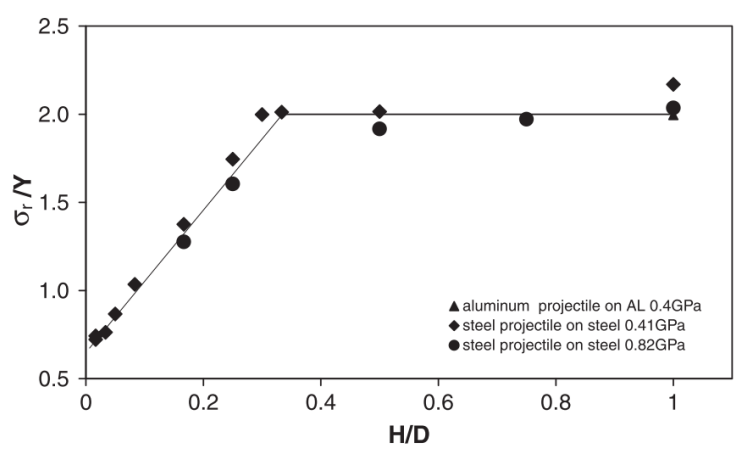

(a)

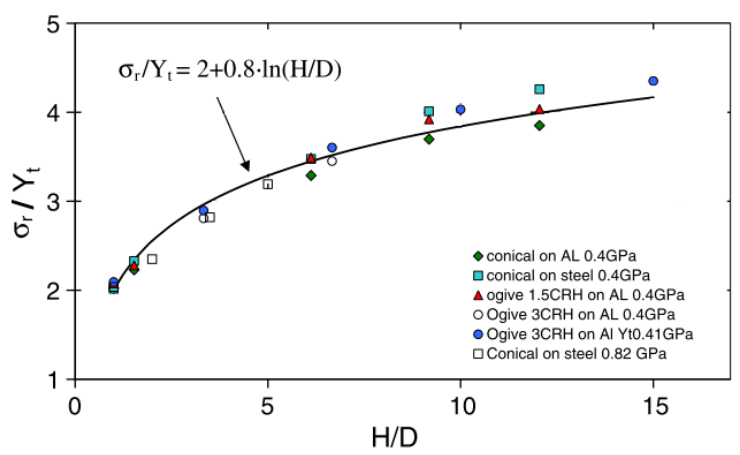

(b)

Figure 25. Analysis of numerical simulation results showing the normalized effective resisting stress for (a) thin to intermediate thickness plates $(H / D \leqslant 1)$ and (b) thick plates $(H / D \geqslant 1)$. Figures reproduced with permission from Rosenberg and Dekel [99].

The results from Fig. 25a allowed Rosenberg and Dekel [99] to determine the point of transition between two failure modes: from dishing to ductile hole enlargement, as seen in Fig. 26. The transition was found to occur at $H / D=0.3$. Plate bending was minimized with increasing thickness. Zaid and Travis [100] found similar results. Another transition can be noted at $H / D=1$, where the normalized effective stress shifted from a constant value $\left(\sigma_{r} / Y_{t}=2.0\right)$ to one having a logarithmic trend. 


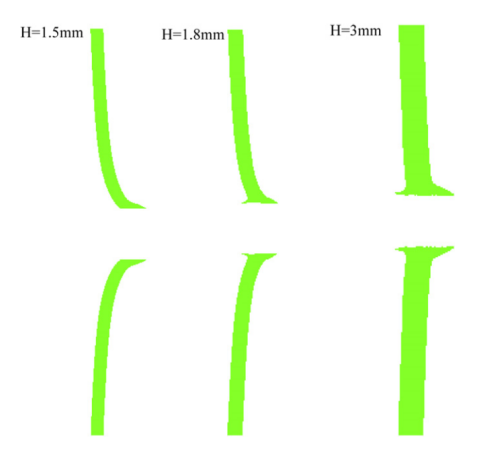

Figure 26. Numerical simulations showing the transition in failure mechanisms of targets having varying thicknesses $(H / D=0.25,0.3$, and 0.5$)$ impacted by a projectile. Figure reproduced with permission from Rosenberg and Dekel [99].

\subsubsection{Penetration of Metals by Blunt Projectiles}

Penetration by a blunt projectile is strongly dependent on the target density, as opposed to perforation by sharp projectiles. It is an ejecta-dominated event that results in some sort of plug being ejected from the back-face surface along with the projectile, as seen in Fig. 23b and 23c. Recht and Ipson [97] developed an analytical model that predicts the residual velocity of a rigid blunt projectile impacting a relatively thin metallic plate at

normal incidence. A plug retaining the circumference of the projectile and the thickness of the plate is assumed to emerge from the target at the same velocity as the projectile due to a perfectly-plastic impact response. Both projectile and plug are assumed to have a constant mass throughout the penetration event [97].

The penetration process is separated in two steps: the first primarily concerns the inertia of the colliding masses, i.e., the deceleration of the projectile and the acceleration of a free plug mass, while the second step considers the circumferential shearing process of said plug. It should be noted that additional deformation energy will be absorbed during the first step since the shear area further contributes a resisting force component to the deceleration force. This portion is conveniently added to the shear energy expended during the second step [97]. Based on the conservation of energy principle, the following equation can be written to describe the penetration process stated above: 


$$
\frac{1}{2} M_{p} V_{i}^{2}=\frac{1}{2}\left(M_{p}+M_{\text {plug }}\right) V_{r}^{2}+E_{f}+W_{s}
$$

where $V_{i}$ is the incident velocity, $V_{r}$ is the residual velocity, $M_{p}$ and $M_{p l u g}$ are the masses of the projectile and the plug, respectively. The term $E_{f}$ accounts for the deformation energy resulting from the plastic impact between the projectile and a free plug (i.e., step one). This can be described by the difference in incident and residual kinetic energies [97]

$$
E_{f}=\frac{1}{2} M_{p} V_{i}^{2}-\frac{1}{2}\left(M_{p}+M_{p l u g}\right) V_{r}^{2}
$$

Considering the conservation of linear momentum between the projectile and a perfectly plastic plug, an expression for $V_{r}$ can be obtained, which is then substituted into the relation above, resulting in Eq. 22:

$$
E_{f}=\left[\frac{M_{p l u g}}{M_{p}+M_{p l u g}}\right] \cdot \frac{1}{2} M_{p} V_{i}^{2}
$$

The $W_{s}$ term considers all the energy or work expended from the shearing and deformation process due to the presence of a shear area (step two) [97]. We can now substitute Eq. 22 into Eq. 20 and rearrange the product to obtain an expression for the work term,

$$
W_{s}=\left[\frac{M_{p}}{M_{p}+M_{\text {plug }}}\right] \cdot \frac{1}{2} M_{p} V_{i}^{2}-\frac{1}{2}\left(M_{p}+M_{p l u g}\right) V_{r}^{2} .
$$

This shear work term is closely related to the dynamic shear strength of the plate and is often approximated as being constant for metals, regardless of impact velocity considerations. The authors supported this assumption during the original derivation of this model by studying high-speed machining experiments, which often tend to experience comparable strain rates relative to high impact penetration events. These studies revealed that the dynamic shear strength of metals remained virtually constant (i.e., independent of strain rate) passed a certain threshold [97]. Consequently, a constant value for $W_{s}$ can be obtained from 
Eq. 23 by considering the lower boundary condition, i.e., $V_{r}=0$ if $V_{i}=V_{b l}$. Therefore,

$$
W_{s}=\left[\frac{M_{p}}{M_{p}+M_{\text {plug }}}\right] \cdot \frac{1}{2} M_{p} V_{b l}^{2} .
$$

We can now substitute Eq. 24 and 22 into Eq. 20 to obtain an expression for residual velocity:

$$
V_{r}=\frac{M_{p}}{M_{p}+M_{p l u g}} \cdot \sqrt{V_{i}^{2}-V_{b l}^{2}}=\frac{1}{1+M_{\text {plug }} / M_{p}} \cdot \sqrt{V_{i}^{2}-V_{b l}^{2}} .
$$

The authors further elaborated on the constant shear work term assumption by explaining that even if the dynamic shear strength of a plate increased dramatically with a given velocity jump, the residual velocity would only vary by a few percentage [97]. A final step can be taken by normalizing Eq. 25 with respect to $V_{b l}$, which allows for the comparison of several material-projectile combinations on the same plot. The result is shown below and will be referred to as the RI plugging model:

$$
\frac{V_{r}}{V_{b l}}=\frac{1}{1+\beta} \cdot \sqrt{\left(\frac{V_{i}}{V_{b l}}\right)^{2}-1} .
$$

The $\beta$ term represents the mass ratio between an ideal plug and the projectile, as described by

$$
\beta=\frac{M_{\text {plug }}}{M_{p}}=\frac{\rho_{\text {plug }} L_{p l u g} A_{r}}{\rho_{p} L_{p}},
$$

where $\rho_{p l u g}$ and $\rho_{p}$ are the densities of the plug and projectile materials while $L_{p l u g}$ and $L_{p}$ are the effective lengths of the plug and projectile, respectively. $A_{r}$ corresponds to the crosssectional area ratio between the plug and the projectile, which is equal to unity for flat-nosed rods [44]. A similar plugging model was also suggested for penetration by hemispherical projectiles [81]: 


$$
\frac{V_{r}}{V_{b l}}=\sqrt{\frac{1}{1+\beta}} \cdot \sqrt{\left(\frac{V_{i}}{V_{b l}}\right)^{2}-1} .
$$

Plugging failure can occur through plastic shearing followed by shear fracture resulting from the growth of coalescence of voids. Borvik et al. [101] studied the penetration behaviour of Weldox 460E steel plates subjected to impact by a flat-nosed projectile. A schematic of the shear plugging event as well as experimental images that demonstrate the void nucleation process are shown in Fig. 27. Voids were seen to initiate at the concentrated shear zone of the left-most image. As penetration persists, these voids grow and coalesce, allowing the crack front to keep growing. This was noted as a typical behaviour for ductile fracture [101].

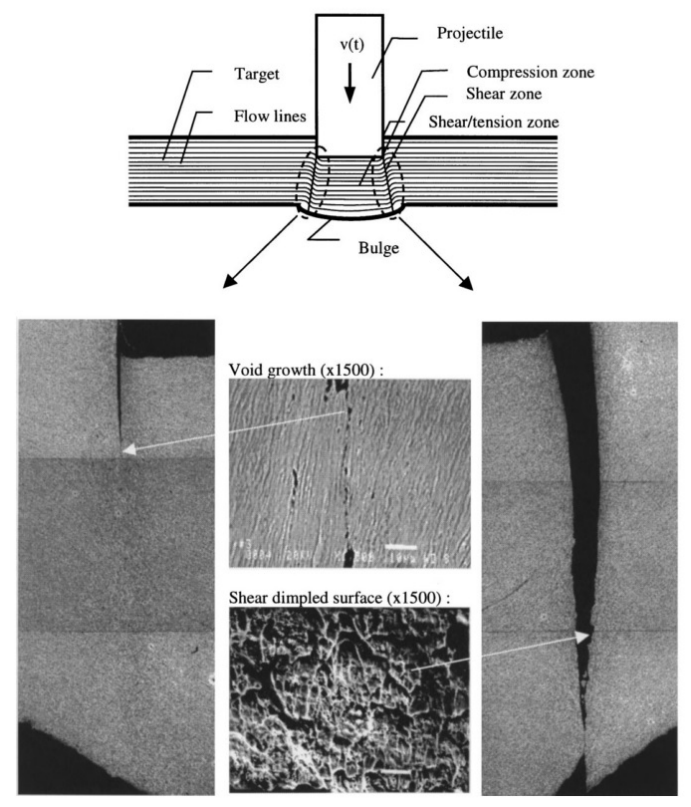

Figure 27. Ductile shear plug formation within a steel plate, resulting in a shear dimpled surface. Figure reproduced with permission from Borvik et al. [101].

On the other hand, some materials respond by catastrophic shearing through narrow bands parallel to the direction of impact $[96,102]$. The latter was first investigated by Zener et al. [103] who claimed that a transition from isothermal to adiabatic deformation may be caused by increased strain rate. Many ductile metals have the capability to strain-harden upon deformation, which distributes the strain throughout the material. During plastic 
deformation at high strain rates, heat is generated locally, thus, creating a temperature gradient whose peek arises from the point of maximum strain. If the strain hardening rate is not able to overcome the rate of decrease in strength resulting from the local temperature increase, the material becomes unstable resulting in a catastrophic local shearing event [104] known as adiabatic shear banding, ASB. Recht [105] studied ultra-high-speed machining experiments and concluded that the dynamic shear strength of a material becomes insensitive to strain rate when ASB is well developed. Titanium and its associated alloys are particularly susceptible to ASB failure. The nominal shear rate required to produce this effect in mild steel is over three magnitudes larger [105]. Figure 28 shows an example of the plugging failure in a titanium alloy resulting from ASB [106]. It can be seen that although the projectile had only penetrated through a small fraction of the plate, the shearing work required to free the plug was nearly achieved.

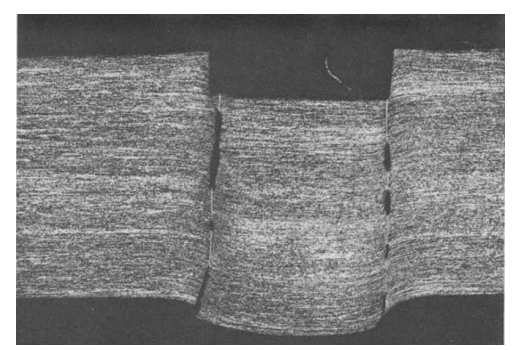

Figure 28. Example of adiabatic shear bands nucleating and coalescing, thus forming a plug within a titanium alloy. Figure reproduced with permission from Woodward [106].

Woodward [107] developed a model that describes the transition in failure mechanism, going from radial flow of the material (DHE) to plugging. The model was based on the notion that the target strength drops dramatically upon the transition to adiabatic shearing (see Fig. 29). A transition point between DHE and ASB was determined by deriving the work required for the projectile to penetrate an additional incremental depth for both failure modes and equating both relations. DHE was found to be energetically favourable for the initial penetration stage, followed by a transition towards plugging at a distance $(\sqrt{3} / 2) D$ from the back surface [107]. Expressions for the work required to penetrate plates through adiabatic shearing have been derived for flat-nosed projectiles [108] as well as for conical 
projectiles [107].

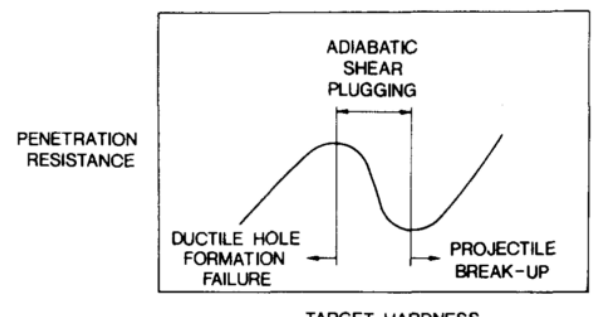

TARGET HARDNESS

Figure 29. Schematic of penetration resistance transitions between ductile hole expansion and adiabatic shearing. Figure reproduced with permission from Woodward [107].

Woodward [102] investigated the plugging response of an aluminum alloy versus a highpurity (low inclusion) aluminum and found that void formation is correlated to the inclusion particles, which result in fracture initiation. It was suggested that targets with low inclusion content can experience significantly more plastic shearing at the plug-target interface prior to shear fracture, as noted in Fig. 30. The images below clearly show a large amount of voids that were formed in the aluminum alloy, which coalesced and caused fracture in the bottom half of the plate. On the other hand, the pure aluminum showed few signs of void formation at the plug-target interface and seems to have necked down to zero cross-section upon ejection from the bottom surface, suggesting improved ductility [102].

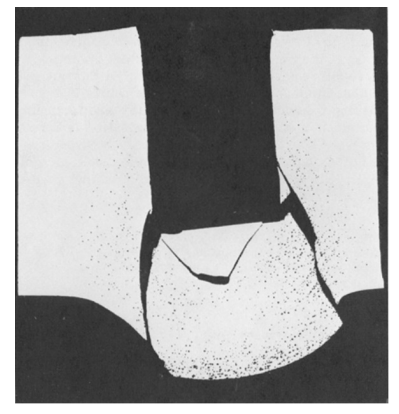

(a)

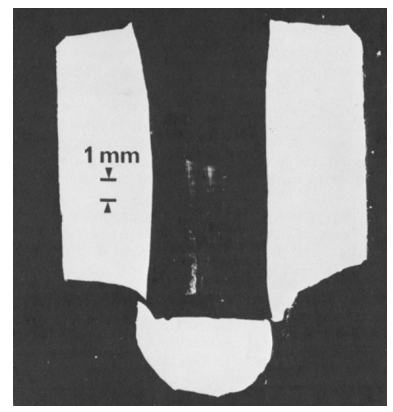

(b)

Figure 30. Difference in plugging response obtained from (a) an aluminum alloy and (b) a high-purity aluminum. Voids were found to nucleate at alloying elements. Figure reproduced with permission from Woodward [102].

Many ballistic studies have been performed on thin sheets of metal. For example, Rusinek et al. [109] modeled the penetration of a hemispherical projectile into 0.8-mm-thick mild 
steel. Frictional effects were found to have a significant influence on the failure mechanism near the ballistic limit: lubricated conditions resulted in a petalling mechanism while the introduction of friction induced plugging failure. As the impact velocity increased further away from the ballistic limit, the influence of friction became minimal and a plugging response was observed for both conditions. The plug diameter was found to be dependent on both the impact velocity and the frictional condition. A more recent numerical investigation was performed by Senthil et al. [90] who studied the evolution of failure mechanisms in thin 2024 aluminum plates impacted by spherical, hemispherical, and blunt projectiles. Petal formation and plugging was observed in both experimental and numerical results, therefore validating the model. Thin mild steel sheets $(1 \mathrm{~mm})$ were subjected to impact by rigid projectiles of different nose shapes and constant mass to study their ballistic response and the influence of projectile geometry [110]. Residual velocity data was obtained from the study and fitted to an empirical model from Recht and Ipson [97] described as

$$
V_{r}=a\left(V_{i}^{k}-V_{b l}^{k}\right)^{1 / k}
$$

where $a$ and $k$ are curve-fitting parameters that are obtained through empirical methods [97]. It should be noted that this model is identical to the previous penetration models from Recht and Ipson [97] if $k=2$ while $a=1$ and $a=\frac{1}{1+\beta}$ for perforation and plugging events, respectively. The hemispherical projectile was found to absorb more energy at low velocities, which is characterized by a higher ballistic limit relative to that of the conical and blunt projectiles. Senthil et al. [90] also reported similar trends in the comparison of hemispherical and blunt projectiles impacting thin aluminum plates. It should be noted that thin plates are prone to absorbing energy through global deformation (dishing), as seen in Fig. 26. The nose shape was found to have a significant role on the failure mode of the thin sheets, although energy absorption was less influenced by its geometry at higher velocities. Similar conclusions were also obtained from the experiments performed by Landkof and Goldsmith $[111]$. 


\subsection{Dense Particle Suspensions under Ballistic Conditions}

A section has been dedicated to the ballistic penetration of dense particle suspensions (DPS) since the experimental work performed on this topic partially consisted of the motivation for the present thesis. Petel et al. [112, 44] performed a series of penetration experiments in order to characterize the ballistic response of DPSs. This was achieved by collecting residual velocity data from 17-grain chisel-nosed FSPs impacting various encapsulated particle suspensions. The experiment schematic in Fig. 31 shows an increased particle volume fraction near the projectile vicinity due to the compressibility of the liquid phase. This may result in the formation of temporary force chains, as discussed in Section 2.2.3.

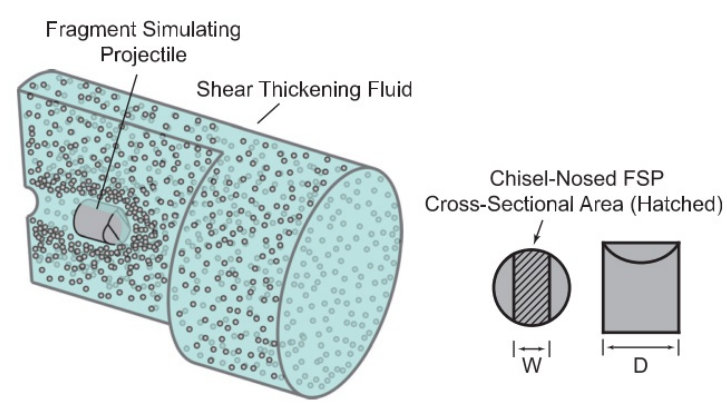

Figure 31. Schematic of ballistic penetration experiments on STF-filled capsules. Figure reproduced with permission from Petel et al. [112].

The ballistic limit velocity was estimated from each residual velocity plot by extrapolating the data to obtain the horizontal axis intercept [44]. The RI plugging model, Eq. 26, was employed to shed light on the penetration mechanics at play. Although the formation of a perfect plug was not evident from the ejecta field, the model was assumed to be representative of the ejecta at the first instant following penetration [44]. The main resistance to penetration was assumed to result from high pressures generated at the flat portion of the chisel nose shown in Fig. 31; only this section contributed to the generation of a plug. The shape factor, $A_{r}=0.58$, was calculated through geometric considerations and used in the RI plugging model, resulting in good agreement for all mixtures (e.g., Fig. 32) [44]. The RI plugging model was found to adequately represent the experimental data of several mixtures, e.g., Fig. 32. This model allows for an assessment of the ejecta field characteristics, however, the 
use of the ballistic limit velocity somewhat limits our ability to discuss the role of material strength [44].

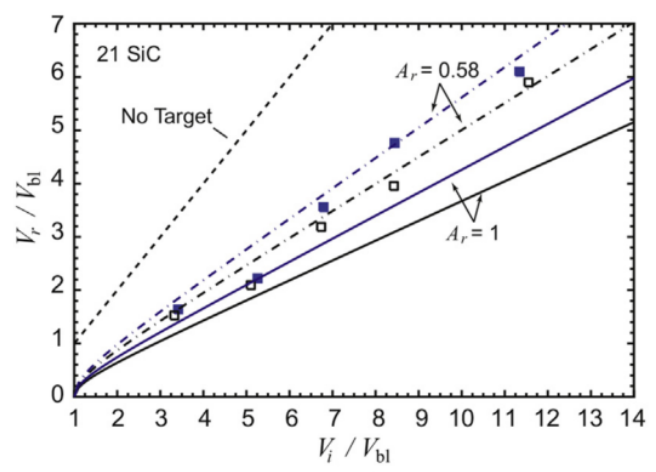

Figure 32. Normalized velocity data and RI plugging model for 21 SiC-EG mixture. Figure reproduced with permission from Petel et al. [44].

A simple analytical model was derived in order to characterize the hydrodynamic (inertial) penetration response of a material impacted by a blunt projectile [112]. Materials can experience a loss of strength when subjected to high enough impact velocities. As a result, the dynamic response of the material becomes entirely dominated by its density. An approach based on the conservation of linear momentum was employed to investigate the dynamic strength of materials [44]. This model assumes that an ideal perfectly-plastic plug is formed upon impact, the projectile remains rigid, and that both projectile and plug maintain a constant mass. The target is also assumed to have no strength. The derivation begins with a momentum balance between the projectile and the plug, i.e.,

$$
\rho_{p} L_{p} V_{i}=\left(\rho_{p} L_{p}+\rho_{\text {plug }} L_{p l u g} A_{r}\right) V_{r},
$$

where $A_{r}$ is the area ratio between the plug and the projectile and is equal to 0.58 and 1.0 for chisel-nosed and flat-nosed projectiles, respectively [44]. Through mathematical manipulation, an expression was derived for the normalized velocity decrement after penetration $[44]$, 


$$
\frac{\Delta V}{V_{i}}=\frac{V_{i}-V_{r}}{V_{i}}=\frac{1}{1+\frac{1}{\beta}} .
$$

The theoretical hydrodynamic limit of a material subjected to blunt impact, Eq. 31, is independent of velocity and is solely influenced by density and projectile geometry. A velocity decrement greater than that of the hydrodynamic limit can be attributed to the dynamic strength of the target [44]. Experimental results demonstrated that ethylene glycol (EG), a 21 vol.\% SiC suspension, and a 54 vol.\% cornstarch-based STF exhibited a hydrodynamic response (i.e., no dynamic strength) [112]. On the other hand, two mixtures were found to significantly deviate from the inertial response, i.e., a 61 vol.\% silica system (61 $\mathrm{SiO}_{2}$ ) and another mixture consisting of 47.6 vol.\% silica and 13.9 vol.\% SiC (61 Mix). Both of these STFs contained a high content of particles with considerable strength and proved to outperform the inertial response described by Eq. 31 at low velocities. The normalized velocity data converged to the hydrodynamic response at higher impact velocities. It was noted that the 61 Mix system maintained a strength-dominated response for a greater range of $V_{i}$ values compared to the $61 \mathrm{SiO}_{2}$ mixture. This was attributed to the superior yield strength of $\mathrm{SiC}$ as compared to the porous silica [112]. Plate impact experiments that were conducted on SiC suspensions experienced shear stresses up to 0.5 GPa [46], clearly demonstrating signs of dynamic material strength. This effect was associated to the formation of force chains resulting in a partially jammed system of particles, as exemplified in Fig. 13. The loss of dynamic strength that occurred passed a certain threshold velocity during the ballistic penetration experiments is expected to be linked to the fracture of particles within the network of force chains, as discussed by Marr et al. [79]. If the dynamic strength of the solid phase is surpassed by the contact stresses, particle fracture would hinder the network of force chains resulting in a loss of strength and recovery of the hydrodynamic response $[112]$.

The effect of solid phase volume fraction on the ballistic performance of particle suspensions can be clearly visualized in Fig. 33 where the normalized velocity results of two 
mixtures (21 $\mathrm{SiC}$ and $61 \mathrm{SiO}_{2}$ ) having equivalent densities are compared to their inertial response (Eq. 31). It is evident that the increased silica content contributed to a dynamic strengthening [44]. Although $\mathrm{SiC}$ has superior strength properties, this type of behaviour was expected due to the increased interparticle contact at higher filler loading. The dilute mixture was not expected to form force chains due to minimal contact between particles, hence the purely inertial response. This is a good example demonstrating that particle strength is only a dominant contributing factor to penetration resistance if there are enough particles present to force significant interparticle contacts [44].

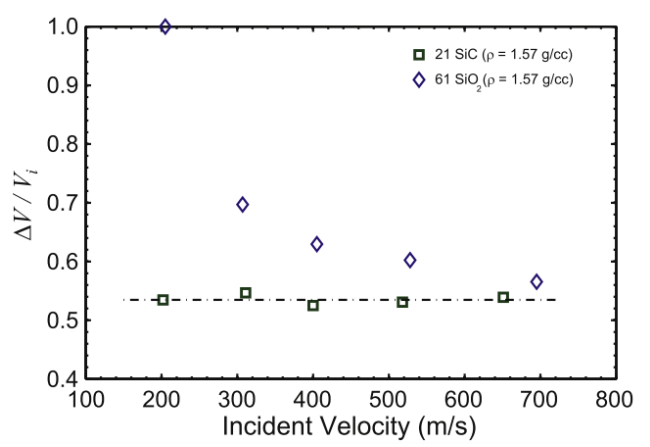

Figure 33. Normalized velocity results of two particle suspensions, exposing the effect of volume fraction on ballistic resistance. Figure reproduced with permission from Petel et al. [44].

It is often advantageous to analyze the response of different materials according to a common baseline in order to obtain better comparison value. A normalized performance parameter $(\kappa)$ was obtained by dividing the normalized velocity decrement (LHS of Eq. 31) by the theoretical hydrodynamic limit (RHS), thus,

$$
\kappa=\frac{V_{i}-V_{r}}{V_{i}}\left(1+\frac{1}{\beta}\right),
$$

where a $\kappa$ value equal to unity is representative of an inertial material response and greater values indicate a dynamic strength contribution [44]. Equation 32 enables direct comparison of the performance of several materials using a single graph while accounting for variations in density. 
Although Eq. 32 measures deviations from the inertial (hydrodynamic) response of a suspension, it does not provide much information concerning variations in resisting shear stress exerted by the target at different impact velocities [113]. The work done during penetration $\left(W_{s}\right)$ was derived from the conservation of energy expression proposed by Recht and Ipson [97] (Eq. 20), resulting in the expression below [113]

$$
W_{s}=\frac{M_{p}}{2}\left[\left(\frac{1}{1+\beta}\right) V_{i}^{2}-(1+\beta) V_{r}^{2}\right] .
$$

Using Eq. 33, an average resistive shear stress $\left(\tau_{a v}\right)$ can be estimated using

$$
\tau_{a v}=\frac{M_{p}}{2 P_{p} L_{p l u g}^{2}}\left[\left(\frac{1}{1+\beta}\right) V_{i}^{2}-(1+\beta) V_{r}^{2}\right],
$$

where $P_{p}$ is the perimeter of the flat portion of the projectile nose. The expression above was used to calculate the average resistive shear stress of several suspensions penetrated by chisel-nosed projectiles [113], which resulted in Fig. 34. It was concluded that the resistive shear stress increased with impact velocity only if the suspended particles were sufficiently strong (e.g., $\mathrm{SiC}$ ) and were loaded to high enough volume fractions to cause a transient jamming response.

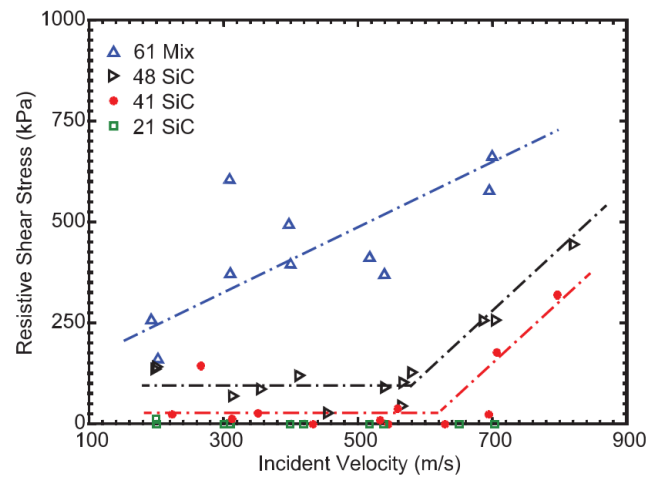

Figure 34. Average resistive shear stress of several dense particle suspensions impacted by chisel-nosed projectiles. Figure reproduced with permission from Petel et al. [113]. 


\subsection{Conclusion}

The present chapter discussed a wide variety of subjects that should be considered prior to pursuing studies in terminal ballistics. These are summarized below while noting the importance of each subject in terms of the present study. The review began by discussing depth of penetration experiments on semi-infinite metal slabs to shed light on some fundamentals of penetration mechanics. It was found that the deep penetration of rigid rods could be characterized by a constant deceleration process. This behaviour translates to a constant resisting force exerted by the target. The constant deceleration assumption is not always valid and was found to be dependent on projectile nose shape and target properties (density and strength), e.g., Fig. 15 and 16, respectively. For a given target-projectile combination, there exists a threshold velocity above which a velocity-dependent inertia term must be added to the resisting force of the target. This phenomenon was called cavitation [87] and results from the projectile transferring additional energy to the target by laterally expanding the material.

Constant deceleration cannot be assumed during the initial penetration stage due to the entrance phase effect. A lower resisting force is generated here since the target material is able to flow backwards, towards the free impact surface (path of least resistance). This was found to be highly dependent on the projectile nose shape since it depends on the indentation process. Similarly, the deceleration process near the back surface of a finitethickness target cannot be constant since the resisting force imposed onto the projectile is reduced. The projectile deceleration process in a finite-thickness target is therefore limited by its free surfaces and should not be assumed constant (see Fig. 21). The conclusions mentioned above directly apply to the ballistic analysis of the present thesis. The finitethickness targets under investigation are not assumed to exert a constant resisting force. Additionally, the PDMS matrix is expected to be highly susceptible to cavitation due to its elastic and low-strength nature.

Several failure mechanisms have been observed at the back surface of targets. These 
depend on factors such as projectile nose shape, the relative thickness of targets $(H / D)$, and material properties such as strength, ductility, etc. The penetration of sharp-nosed projectiles generally involves the DHE process. Recht and Ipson [97] derived an analytical model based on energy conservation principles which assumed that all material was pushed laterally aside during penetration (DHE). In contrast, flat-nosed projectiles typically penetrate targets through a shear plugging mechanism, therefore pushing material axially ahead of the nose. A similar analytical model was derived by conserving energy between plug and projectile [97]. Both RI perforation and plugging models have been normalized with respect to $V_{b l}$ in order to account for material properties and nose shape effects. This resulted in Eq. 18 and 26, respectively.

Some metallic targets were found to experience catastrophic shear (plugging) failure due to the formation of adiabatic shear bands. Characteristics that make a material prone to such behaviour are high strength, low work hardening, and a high thermal softening rate [106]. Alternatively, plugging failure can occur through plastic shearing followed by unstable shear fracture. The transition to fracture was associated to the growth and coalescence of voids which have been previously found to nucleate in the vicinity of large alloying inclusions [102]. Pure metals exhibited significantly more plastic shearing prior to plug ejection, as seen in Fig. 30. Consequently, the work invested by the projectile during penetration depends on the failure mechanisms of the target material, which will be investigated later on.

Ballistic studies concerning the penetration response of DPS [112, 44, 113] were summarized in Section 3.3 due to their relevance to the present thesis. The RI plugging model was used to represent the material response, Eq. 26, however a shape factor $A_{r}=0.58$ was introduced to account for the narrow flat portion of the FSPs. A model describing the hydrodynamic (inertial) response of a material was developed by considering the conservation of linear momentum between a flat-nosed projectile and an ideal plug, Eq. 31. A normalized performance parameter, $\kappa$, was derived from this hydrodynamic model to rank the dynamic performance of several suspensions on a single graph while accounting for differences in den- 
sity. Signs of significant dynamic strength were observed for suspensions having high particle concentrations (61 vol.\%). These were attributed to shock-induced microstructural changes causing a network of interconnected particles (force chains). The dynamic response of suspensions was dominated by the filler material strength once excessive particle collisions were present. An expression for the resistive shear stress was derived as an attempt to quantify the dynamic material strength while disregarding the dominating contribution from inertia. The dynamic shear strength was found to be dependent of particle concentration, material strength, and impact velocity, see Fig. 34 .

A similar experimental and analytical procedure was employed in the present ballistic study. A description of the experimental process is provided in the following chapter. The models discussed above were used to assess the dynamic performance of the various particlefilled elastomeric systems; the results are presented and discussed in Chapter 6. The general applicability of these equations for the specified materials will also be evaluated. A qualitative post-mortem analysis is employed in Chapter 7 to further assess the failure mechanisms of composites. 


\section{Experimental Details}

Particle-filled elastomer composites were prepared in-house for mechanical characterization under quasi-static and ballistic conditions. Quasi-static uniaxial tensile and shear testing was conducted at Carleton University in order to provide limited mechanical characterization of composites having varying inclusion properties. Ballistic penetration experiments were performed at the Defence Research and Development Canada (DRDC) facility in Valcartier, Quebec, in collaboration and with the assistance of DRDC personnel. The two-phase system under consideration consisted of an elastomeric matrix embedded with micron-sized ceramic particles at several concentrations. The vast majority of ceramic powders were sourced from Panadyne Inc. (Montgomery, PA).

A polydimethylsiloxane (PDMS) thermosetting elastomer, i.e., Sylgard 184 (Dow Corning), was selected as the polymer matrix for the present study. This elastomer was selected based on its relatively inexpensive and simple manufacturing process, its ability to cure un-

der atmospheric conditions, and the availability of shock compression data [64]. Sylgard 184 was used as a representative elastomer through which we can investigate the role of hard inclusions within a flexible thermosetting polymer matrix. The following sections provide an in-depth description of the experimental work undertaken. A description of the particles considered in the present thesis is provided next, followed by details of the procedure used for composite synthesis. The objective of quasi-static experiments was to collect forcedisplacement data from MTS testing while ballistic experiments measured the incident and residual velocities of several projectile-target combinations.

\subsection{Characterization of Ceramic Powders}

Scanning electron microscopy (SEM) was used to characterize the ceramic powders considered in this study, as seen in Fig. 35. The contrasting subsets of powders were chosen in order to compare the effect of varying materials (Fig. 35a - 35d) as well as particle mor- 
phology (Fig. 35d and 35e). All powders had relatively similar characteristic lengths within the lower micron range, allowing us to minimize particle size effects. The irregular-shaped particles (Fig. 35a - 35d) were blocky in nature with jagged edges, rough surfaces, and aspect ratios ranging from approximately 1 to 10 while the spherical powder (Fig. 35e) appeared to have smooth surfaces and an aspect ratio of unity. All powders were employed as-received, i.e., having no surface treatment. Table 2 lists typical mechanical property values for the materials used in the present study.

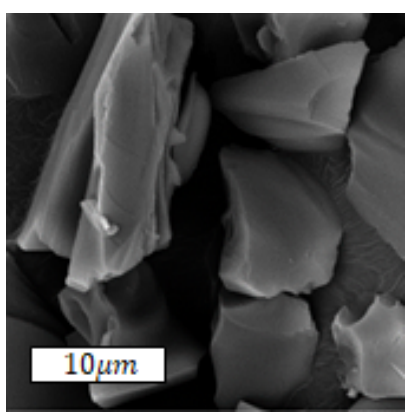

(a)

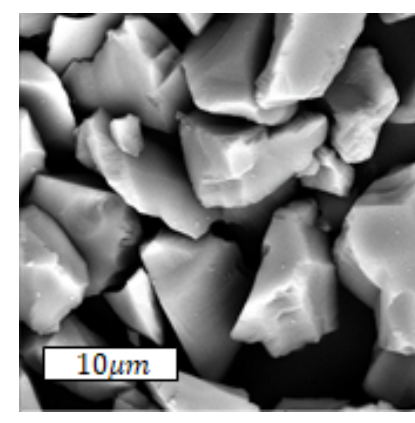

(b)

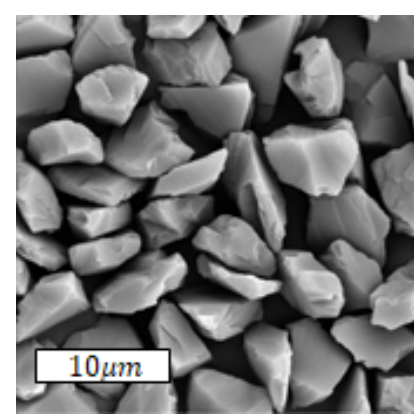

(c)

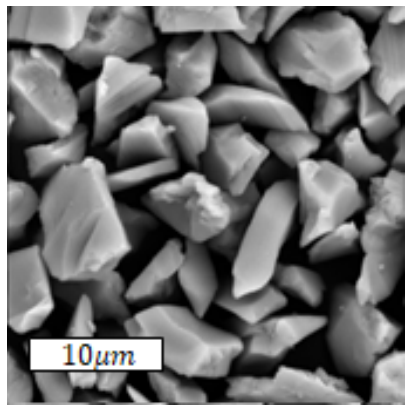

(d)

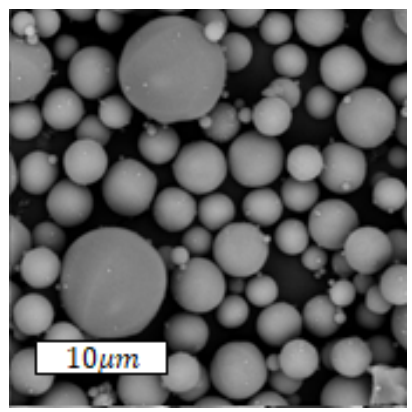

(e)

Figure 35. SEM images of powders: (a) boron carbide $\left(\mathrm{B}_{4} \mathrm{C}\right)$, (b) silicon carbide $(\mathrm{SiC})$, (c) diamond , (d) alumina $\left(\mathrm{Al}_{2} \mathrm{O}_{3}\right)$ - irregular, and (e) alumina $\left(\mathrm{Al}_{2} \mathrm{O}_{3}\right)$ - spherical.

Table 2. Typical mechanical property values of the materials under investigation.

\begin{tabular}{lccc}
\hline Material & Density $\left(\mathrm{g} / \mathrm{cm}^{3}\right)$ & Young's Modulus $(\mathrm{GPa})$ & References \\
\hline PDMS (Sylgard 184) & 1.03 & $1.3-3.0 \times 10^{-3}$ & {$[62,58]$} \\
$\mathrm{B}_{4} \mathrm{C}$ & 2.52 & $290-450$ & {$[114]$} \\
$\mathrm{SiC}$ & 3.21 & $410-441$ & {$[114]$} \\
Diamond & 3.52 & $700-1,200$ & {$[13]$} \\
$\mathrm{Al}_{2} \mathrm{O}_{3}$ & 3.95 & $345-409$ & {$[114]$} \\
\hline
\end{tabular}

Details concerning the size distribution of the particles within the powders were obtained 
directly from Panadyne Inc. and are presented in Table 3.

Table 3. Particle size distribution data of ceramic powders purchased from Panadyne Inc.

\begin{tabular}{lccc}
\hline Powder Material & $\mathrm{D}_{3}(\mu m)$ & $\mathrm{D}_{50}(\mu m)$ & $\mathrm{D}_{94}(\mu m)$ \\
\hline $\mathrm{B}_{4} \mathrm{C}$ & 21.04 & 13.15 & 6.86 \\
$\mathrm{SiC}$ & 16.63 & 9.01 & 4.3 \\
Diamond & $8.40\left(\mathrm{D}_{6}\right)$ & 5.59 & $4.18\left(\mathrm{D}_{90}\right)$ \\
$\mathrm{Al}_{2} \mathrm{O}_{3}$ (irregular) & 6.95 & 4.58 & 2.98 \\
$\mathrm{Al}_{2} \mathrm{O}_{3}$ (spherical) & 10.98 & 5.34 & 2.89 \\
\hline
\end{tabular}

\subsection{Composite Specimen Preparation Methodology}

Sylgard 184 was supplied as a two-part liquid component kit: a pre-polymer base and a curing agent having a recommended mixing ratio of 10:1 (by weight), as per manufacturer specifications [62]. The PDMS matrix enabled the preparation of test specimens through a cast moulding process. The moulds used for this study will be presented in the following sections. All ballistic capsules were cured at room temperature while tensile and shear specimens were both heat-cured (HC) and room-temperature-cured (RTC). Although oven temperatures were not digitally recorded during the heat-curing process, analog thermometers were constantly monitored and the heat input dial adjusted in order to obtain similar curing temperature histories for all sample sets. The manufacturing procedure used to prepare particulate composites is described below:

1. Mechanically mix the ceramic powder into the pre-polymer base liquid in order to obtain a uniform particle distribution at a specified volume fraction

2. Heat the mixture in a conventional oven at approximately $100^{\circ} \mathrm{C}$ for 12 minutes to decrease the viscosity prior to the sonication stage

3. Place the mixture in a ultrasonic water bath for 20-45 minutes as an attempt to break up aggregates and obtain a more homogeneous particle distribution within the liquid 
4. Once the mixture has returned to room temperature, integrate curing agent into the solution at a 10:1 base-curing agent mix ratio, followed by thorough mixing

5. Desiccate the solution for approximately 1.5 hours to remove trapped air and obtain a void-free particulate system

6. Transfer mixture to each respective mould and manually remove resulting trapped air bubbles near the surface

7. Set ballistic samples/moulds onto flat surface and allow to cure under atmospheric conditions for 48 hours

8. Place tensile and shear samples/moulds in conventional oven and allow to cure at $100-120^{\circ} \mathrm{C}$ for 44 minutes. Alternatively, cure samples at room temperature (step 7).

It should be noted that a maximum particle volume fraction of $33.3 \%$ was achieved while maintaining a void-free composite. Further increases in particle content resulted in an excessively viscous liquid with questionable homogeneity. Upon desiccation of the mixture, a significant amount of air bubbles remained trapped within the system. This was attributed to a combination of two factors, i.e., the highly viscous nature of the mixture impeding the trapped air from reaching the free surface and the allowable working time of the PDMS prior to its solidification. Therefore, increased particle concentrations were limited by both the choice of matrix and the manufacturing procedure. For similar reasons, no mould lids were used during the casting process since trapped air tended to accumulate under these surfaces and could not escape prior to curing of the PDMS. Consequently, the moulds were left open to atmosphere.

\subsection{Quasi-static Testing Description and Setup}

Uniaxial tension and shear punch experiments were performed using hydraulic MTS machines at two quasi-static strain rates in order to investigate the behaviour of several particulate 
composites and their baseline elastomer matrix. Moreover, this allowed for a limited discussion on the strain rate sensitivity of the materials considered while revealing their associated trends. Force-displacement data were collected from the MTS software in order to quantify the mechanical properties of the systems. Six samples were tested for each material-loading combination, although some data was omitted due to improper failure.

\subsubsection{Shear Testing and Specimen Details}

Shear strength experiments were conducted using a steel shear punch tool (Fig. 36) that was manufactured in-house according to the specifications described in ASTM D732-10 [115]. Disk-shaped specimens conforming to the dimension requirements of the ASTM standard were cast from a 6061-T6 aluminum mould complete with shoulder bolt inserts (for center holes). The mould was manufactured using a CNC milling machine and is shown in Fig. 37.

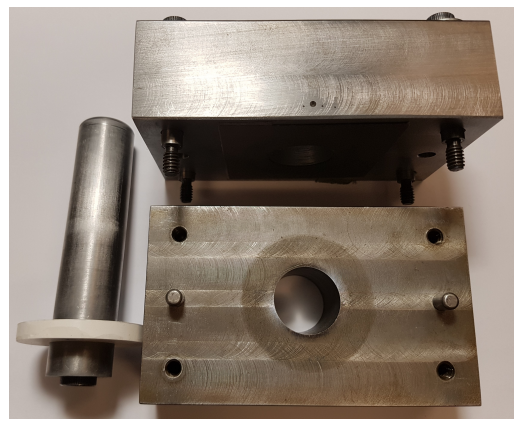

(a)

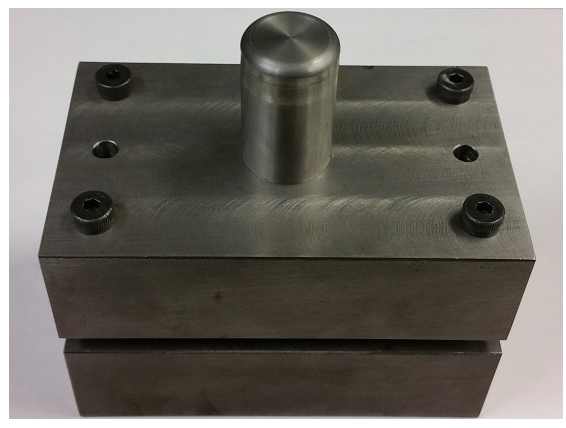

(b)

Figure 36. Steel shear punch tool: (a) open die showing punch with mounted specimen and (b) closed-die assembly. Apparatus manufactured according to ASTM D732-10 [115].

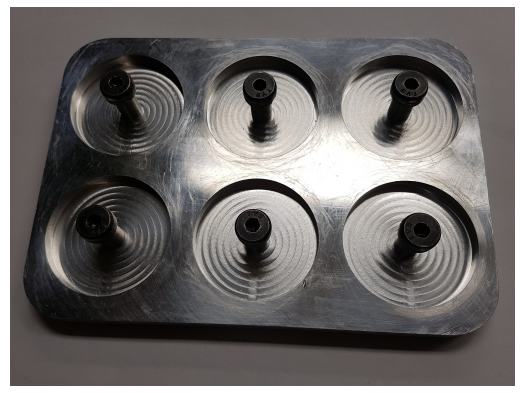

Figure 37. Aluminum shear sample mould - designed according to ASTM D732-10 [115]. 
Six disk-shaped specimens (thickness $\approx 0.25$ in) were prepared for each testing scenario. The average thickness of each sample was calculated through measurements obtained from each disk quadrant using digital calipers. Care had to be taken when measuring the thickness of the highly elastic disks since minimal force was required to compress the samples, resulting in inaccurate measurements. As per the ASTM standard, individual disks were lightly clamped against the flat circular shear punch and a backing plate, as seen in Fig. 36a, to avoid sample bending or stretching upon loading. This backing plate was used in an attempt to obtain a pure shear response. The punch was then placed into the penetration channel of the die prior to securing the two halves with shoulder bolts, thus, constraining the sample from transverse slippage, as seen in Fig. 36b. Once again, care was taken to not over-tighten the samples, which would otherwise result in an apparent increase in stiffness upon loading. The testing apparatus was then placed under a 25-kN MTS Bionix press, as demonstrated in Fig. 38. The MTS was configured to travel at a constant punch displacement rate $\left(V_{p}\right)$ of either $1.3 \mathrm{~mm} / \mathrm{min}$ or $3000 \mathrm{~mm} / \mathrm{min}$. The definition of shear strain rate $(\dot{\gamma})$ was adopted from Ref. [116], i.e.,

$$
\dot{\gamma}=\frac{V_{p}}{2 W}
$$

where $W$ is the clearance between the punch and the die wall and was measured to be approximately $0.05 \mathrm{~mm}$. Therefore, the resulting shear strain rates were estimated as $2.13 \cdot 10^{-1}$ and $4.92 \cdot 10^{2} s^{-1}$, respectively. The hydraulic press forced the punch through the specimen while recording force-displacement data and extracting a shear plug from the sample.

\subsubsection{Tensile Testing and Specimen Details}

A 25-kN hydraulic MTS 810 testing system was used to perform single-pull-to-failure tensile experiments as per ASTM D412-06a [117]. Dumbbell specimens were designed according to the type $\mathrm{C}$ geometry specifications of the ASTM standard. Composites were tested at two constant displacement rates, i.e., $0.5 \mathrm{~mm} / \mathrm{s}$ and $100 \mathrm{~mm} / \mathrm{s}$, resulting in strain rates of $8.18 \cdot 10^{-3}$ and $1.64 s^{-1}$, respectively. The force-displacement data was then converted to 


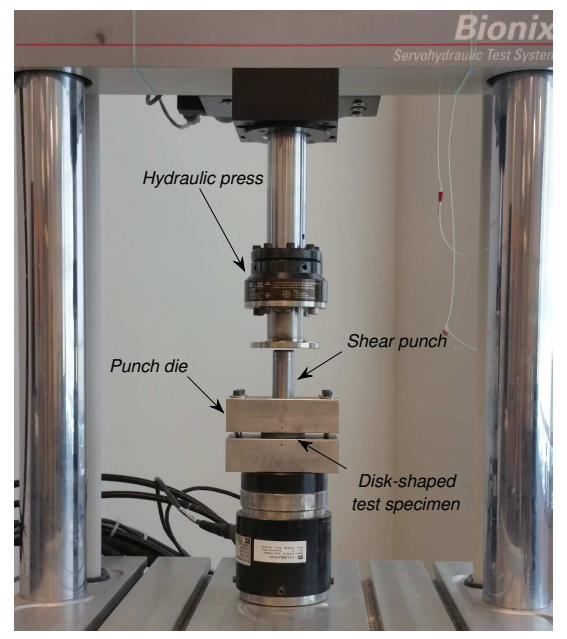

Figure 38. MTS and apparatus mounting setup for shear punch testing.

engineering stress and strain.

Prior cast moulding techniques seemed to generate imperfections within the material when peeling off the dumbbell specimens from the backing plate/mould, hence initiating premature failure. This was resolved by employing a combination of cast moulding and the use of a cutting die. Flat rectangular sheets of composite material (thickness $\approx 4 \mathrm{~mm}$ ) were cast from the aluminum moulds shown in Fig. 39. A steel rule cutting die was used in conjunction with a manual press to shear the dumbbell-shaped samples from the sheets. The cutting die, complete with rubberized foam padding (for easy sample extraction), was manufactured by Key Dies Inc. and is shown in Fig. 40. The dumbbell samples produced by the cutting die had much cleaner edges than their cast counterparts and improved the repeatability of the results. Once the samples were cut, the average thickness and width of each test section was calculated from three measurements that were carefully obtained using digital calipers.

Support tabs were attached to both sides of the dumbbell extremities using double-sided adhesive tape prior to testing. This allowed the hydraulic grips of the MTS machine to apply a uniform pressure distribution without damaging the sample upon clamping of the ends. The gauge length used to calculate engineering strain and strain rate was measured as the 


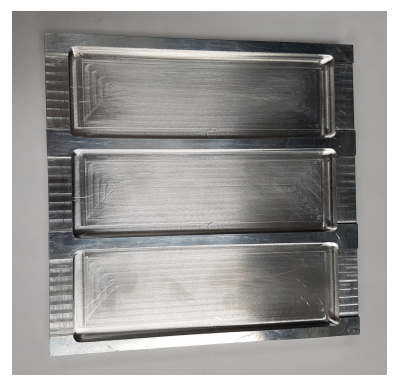

Figure 39. Aluminum tensile sheet mould.

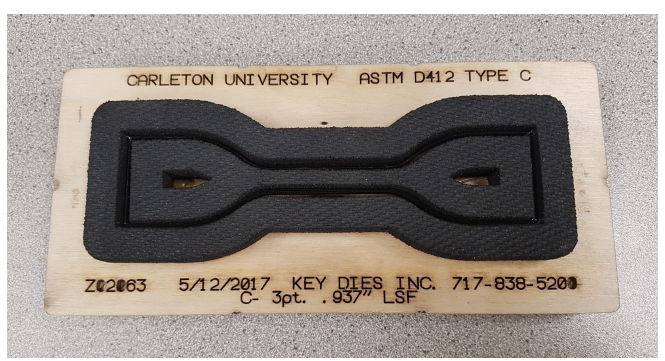

Figure 40. Steel rule dumbbell cutting die - manufactured by Key Dies Inc.

distance between these support tabs, resulting in an average value of $61.14 \mathrm{~mm}$. Alignment tabs were adhered to one side of each set of the MTS grips to assist in proper mounting of the specimens and minimize data scatter by assuring uniaxial tension. All tabs were 3D-printed from ABSplus-P430 using an in-house Dimension SST 1200es printer. The use of support tabs along with the experimental setup for tensile testing is shown in Fig. 41.

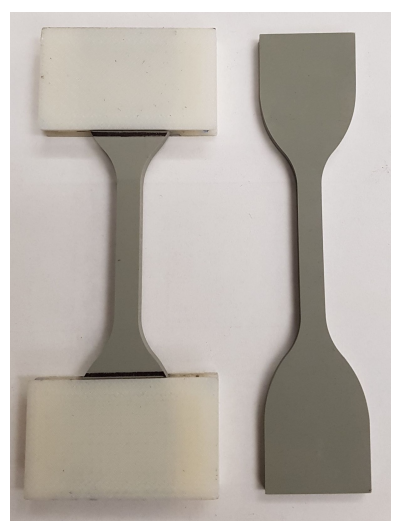

(a)

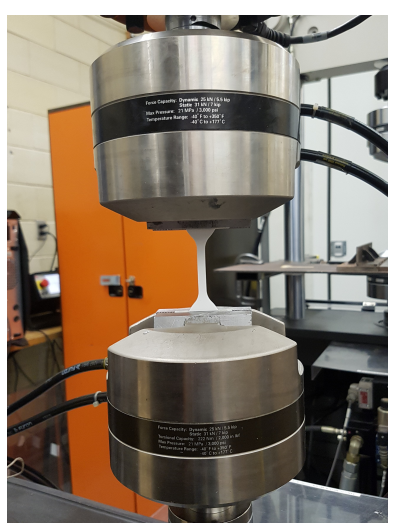

(b)

Figure 41. Tensile experiment setup: (a) dumbbell specimen with support tabs and (b) MTS configuration. 


\subsection{Ballistic Testing Description and Setup}

Ballistic testing of particulate composites was performed at DRDC Valcartier facilities through the use of a single-stage smooth bore light gas gun, Fig. 42. Ballistic targets were cast in standard ABS plumbing pipes (inner diameter $=40 \mathrm{~mm}$ ) that were machined to a consistent height using a lathe. The cylindrical targets were secured in mounting rings and then penetrated by 16-grain projectiles at normal incidence. A detailed schematic along with an image of the experimental setup is provided in Fig. 43. Incident and residual velocities ( $V_{i}$ and $V_{r}$, respectively) were measured using high-speed videography techniques at 25,000 or 35,000 fps. The distance traveled by the projectile between two consecutive frames was compared to a baseline scaling image in order to calculate the instantaneous projectile velocity before and after penetration.

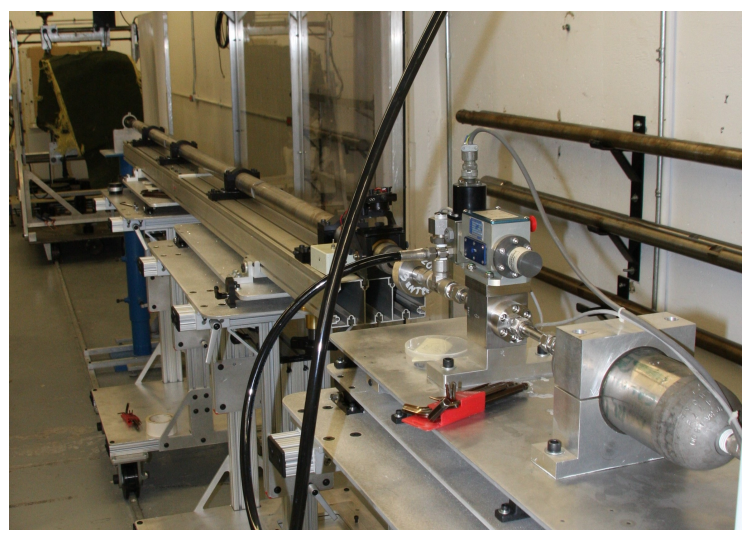

Figure 42. Single-stage smooth-bore light gas gun (DRDC Valcartier).

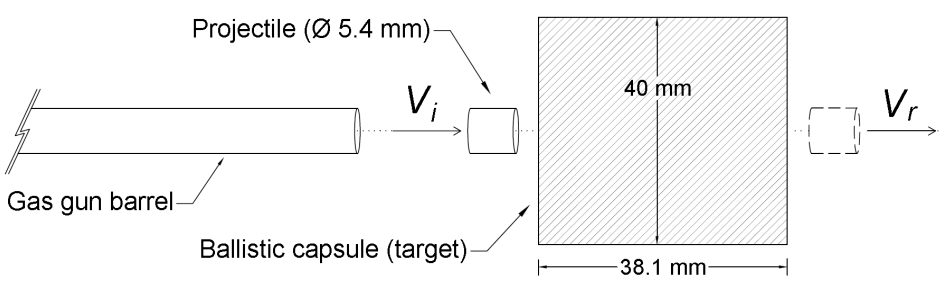

(a)

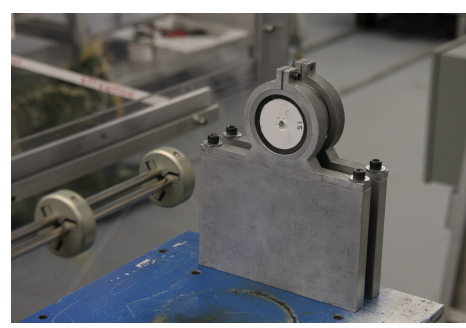

(b)

Figure 43. Experimental setup for ballistic testing: (a) schematic and (b) photograph.

Projectiles (diameter $=0.213_{-0.001}^{+0.0005}$ in) of varying nose geometries were manufactured 
from 4340 steel and heat-treated to a hardness of approximately 30 HRC. Four nose geometries were tested; the shank length of the projectiles were adjusted depending on the nose shape in order to maintain a relatively constant mass of 16 grains $(1.04 \mathrm{~g})$. Figure 44 shows the different projectile types used in the present study. The large majority of ballistic testing used flat-nosed projectiles, i.e., right-angled circular cylinders (RCC). The use of conical projectiles was limited due to issues concerning excessive yaw prior to impact.

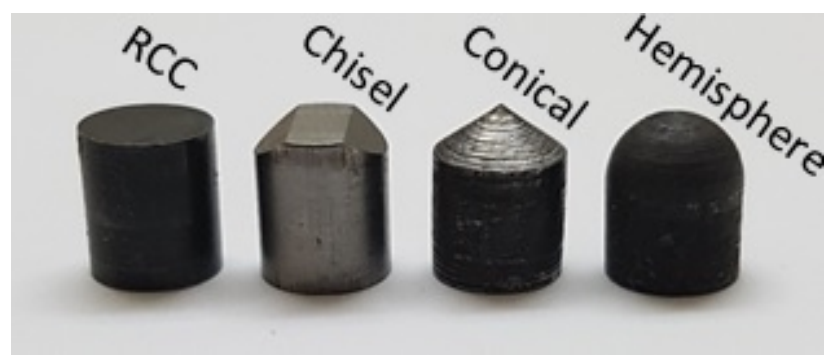

Figure 44. Various 16-grain steel fragment simulating projectiles tested in the present study.

A significant amount of data was extracted from MTS testing and ballistic penetration experiments, which enabled the mechanical characterization of particle-filled elastomers under quasi-static and ballistic conditions. Results from shear and tensile testing are presented in the following chapter and discussed in terms of the trends observed in literature. The ballistic penetration data of the systems under investigation are analyzed in Chapter 6 . 


\section{Mechanical Characterization: Quasi-static Results and Discussion}

Quasi-static mechanical characterization of elastomeric systems was used as a preliminary tool to evaluate material behaviour and to discuss the role of the particle reinforcements under different loading conditions. Although the dynamic and ballistic behaviour of materials is dominated by inertial effects, low-strain-rate experiments can provide valuable information by observing trends in composite strength, stiffness, etc. These properties were tracked as the composite parameters varied in terms of particle concentration, material type, and particle morphology. Signs of weak particle-matrix interface strength can also be perceived by comparing the material response to particulate models found in Chapter 2.

The particulate composites under consideration have been characterized under shear and uniaxial tension conditions. Shear testing was performed using a punch-press operation to extract a plug from a relatively thin plate. Since inertia has a dominating influence on the force required for ballistic penetration, quasi-static shear punching can be thought of as a special case of plate penetration, considering geometry and material strength only [97]. A complex stress state can be expected upon penetration of elastomeric materials, thus justifying the rationale behind tensile characterization. A limited strain-rate sensitivity analysis is provided for both loading conditions to further study the effect of particle integration. The general trends could assist (to a certain extent) in extrapolation of the dynamic behaviour to higher loading rates.

The objective of quasi-static testing is to provide a foundation for the discussion of the ballistic response of particle-filled elastomers, which is covered in the following chapters. Materials were prepared according to Section 4.2. The experimental testing procedure was previously discussed in Section 4.3. 


\subsection{Baseline Characterization: Neat PDMS Elastomer}

Prior to studying the response of particle-filled elastomers, mechanical characterization of the neat PDMS matrix, Sylgard 184, is in order. Once completed, the role of particles can be assessed for composites loaded in tension and shear.

\subsubsection{Tensile Testing}

Tensile dumbbell coupons consisting of Sylgard 184 were prepared and loaded under uniaxial tension, resulting in force-displacement data that were then converted into engineering stress and strain values. The samples were originally cured at room temperature using a cast-moulding technique, however, the material was found to be extremely sensitive to imperfections resulting from this procedure, which often led to premature failure. The intrinsic elasticity of the material also made it difficult to properly mount samples within the MTS clamps and accurately assign a zero-strain origin. Mounting issues could have generated a complex stress state within the samples, which may be a cause for the lack of consistency in published strength values found throughout the literature, e.g., $[58,56,62]$. In order to improve the repeatability of experiments, the PDMS was heat-cured to obtain an increased stiffness [58], which helped the mounting process. A cutting die was also used to produce the dumbbells instead of the moulding technique in order to minimize imperfections upon sample-removal. More details concerning the experimental procedure are found in Section 4.2 and 4.3.2.

A typical stress-strain curve for the samples cured at room temperature and under heat (RTC and HC, respectively) is found in Fig. 45. The constant strain rate $\dot{\epsilon}$ was set to $8.18 \cdot 10^{-3} \mathrm{~s}^{-1}$. The general trends in material behaviour observed in HC samples were similar to those observed by Schneider et al. [57], although different strength and failurestrain values were recorded (see Fig. 6). This was expected since the loading rates were different and their curing temperature was not reported. It should be noted that the strain values from Schneider et al. $[56,57]$ were modified using a correction factor in order to 
account for the elongation occurring within the shouldered extremities of the dumbbells, i.e., outside of the region of interest. No such correction factor was incorporated in our results since the aim of the study was not to accurately quantify the strain-to-failure $\epsilon_{u t s}$, but rather observe the trends in comparison to those of the composites under investigation.

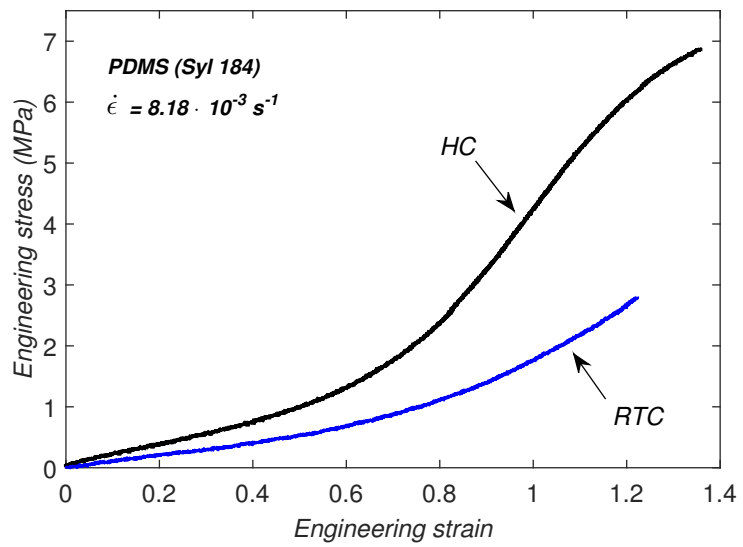

Figure 45. Tensile stress-strain curves of the PDMS elastomer given two curing conditions $\left(\dot{\epsilon}=8.18 \cdot 10^{-3} s^{-1}\right)$.

As seen in Fig. 45, the tensile response of the PDMS-HC was found to have a sufficiently linear stress-strain relationship up to a certain strain value, followed by a non-linear increase in stiffness (strain hardening), which was assumed to result from the alignment of polymer chains. An inflection point occurred at higher strains resulting in a yielding behaviour prior to ultimate failure. No such yielding was found for the PDMS-RTC, although all other trends were present. This could be a sign of premature failure initiated by microscopic tears within the dumbbell resulting from sample removal during the casting process. Another explanation for the yielding behaviour arises from considering potential slip between the MTS jaws and the flexible samples. The jaws secured the dumbbells in position using support tabs, as discussed in Section 4.3.2. Double-sided adhesive tape was used to bond the sample and the tabs, although this was primarily for mounting purposes. Clamping pressure was relatively low due to the high elasticity of the material, which could have caused slipping between the sample and the support tabs. Such an event would manifest itself as a yield-like response on the stress-strain curve. Additional testing involving permanently-bonded support tabs 
would be required to verify this behaviour.

Once we obtained a reproducible stress-strain curve for the heat-cured PDMS, a strain rate sensitivity analysis was performed by increasing the displacement velocity of the MTS jaws to $100 \mathrm{~mm} / \mathrm{s}\left(\dot{\epsilon}=1.64 \mathrm{~s}^{-1}\right)$. The results are found in Fig. 46 in comparison to those obtained from the previous experiment.

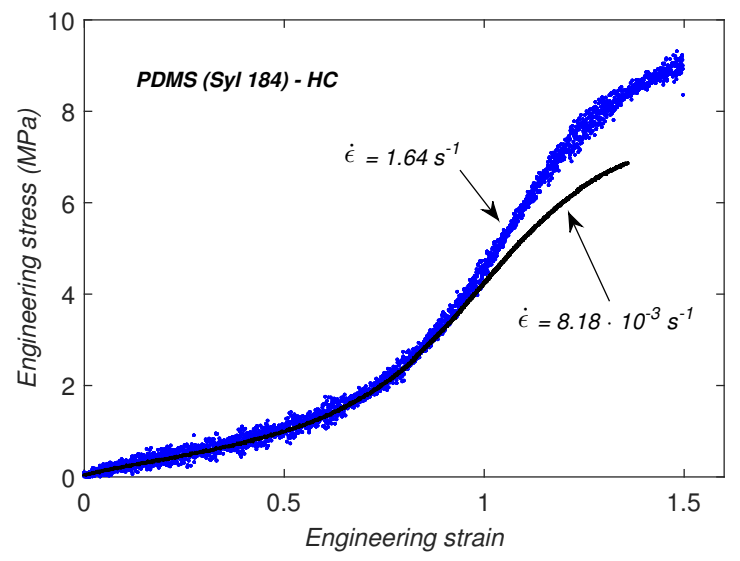

Figure 46. Tensile stress-strain curves of heat-cured PDMS loaded at two strain rates $\dot{\epsilon}$.

The behaviour of the PDMS was found to be unaffected by the increased strain rate up until a strain value of approximately 75\%, which coincides with the non-linear transition zone. It appears that a higher strain rate may have promoted further strain hardening. The ultimate tensile strength and $\epsilon_{u t s}$ both increased as a result.

\subsubsection{Shear Testing}

Shear punch experiments were conducted on neat PDMS elastomer disks as detailed in Section 4.3.1 to obtain a baseline for comparison against the elastomeric composites. Shear stress measurements were obtained at two loading rates, i.e., $1.3 \mathrm{~mm} / \mathrm{min}\left(\dot{\gamma} \approx 2.13 \cdot 10^{-1}\right.$ $\left.s^{-1}\right)$ and $3000 \mathrm{~mm} / \mathrm{min}\left(\dot{\gamma} \approx 4.92 \cdot 10^{2} s^{-1}\right)$. Two curing temperatures were chosen to investigate its influence on the shear response of the PDMS. The elastomers were cured at ambient room temperature (RTC) or heat-cured (HC) at approximately $110{ }^{\circ} \mathrm{C}$. Figure 47 shows typical shear stress curves obtained through testing at the two punch velocities in order to highlight the effect of strain rate for both curing methods. Shear stresses were 
plotted against the relative penetration depth, which is defined as the distance traveled by the punch normalized by the total sample thickness. It should be noted that this is not necessarily representative of the local strain experienced by the specimen.

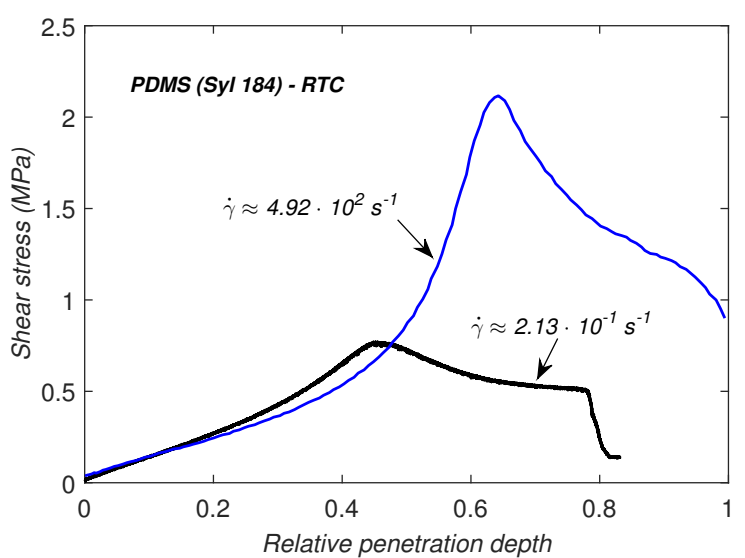

(a)

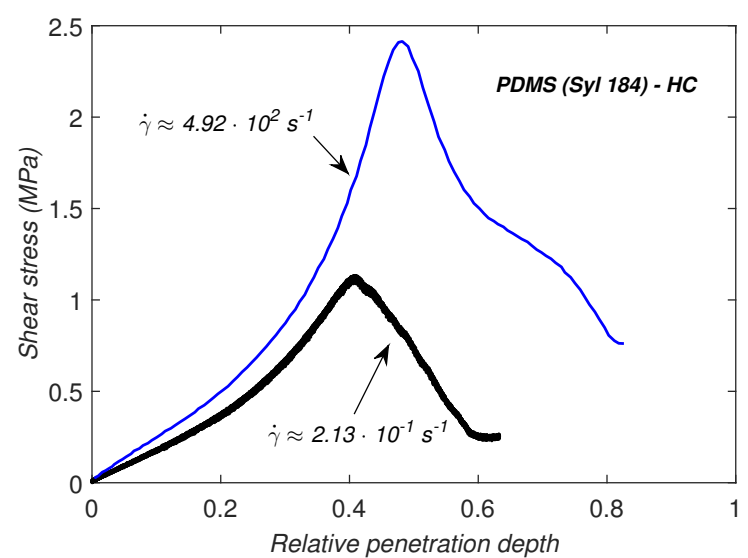

(b)

Figure 47. Shear results for neat PDMS demonstrating the effect of loading rate for two curing conditions: (a) room temperature cure (RTC) and (b) heat cure (HC).

The neat PDMS was found to be highly sensitive to changes in strain rate, as evidenced by a significant increase in shear strength for both curing methods when sheared at higher velocities. The effect of curing methods at a constant shearing rate can be visualized in Fig. 48. Similar to the tensile results, an increase in stiffness and shear strength was observed as the curing temperature increased. Shear failure initiation occurred at the point of peak load (shear strength), which was validated through visual inspection of samples. Once shear initiated, the resisting stress exerted by the elastomer reduced dramatically due to a combination of shear and tensile failure. The punch depth that initiated shear failure was not strongly affected by curing temperature at the lower shear rate, Fig. 48a. The shear initiation depth was much more dependent on curing temperature at higher strain rates. For example, Fig. 48b shows that heat-cured samples experienced earlier shear initiation. 


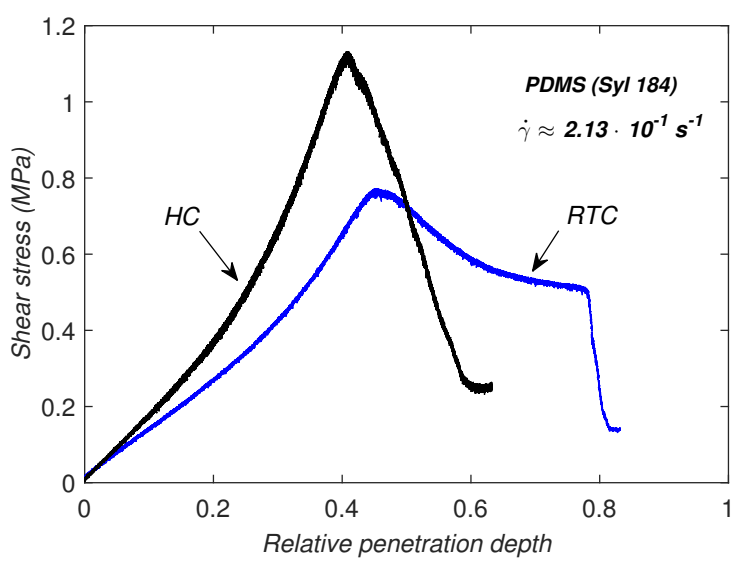

(a)

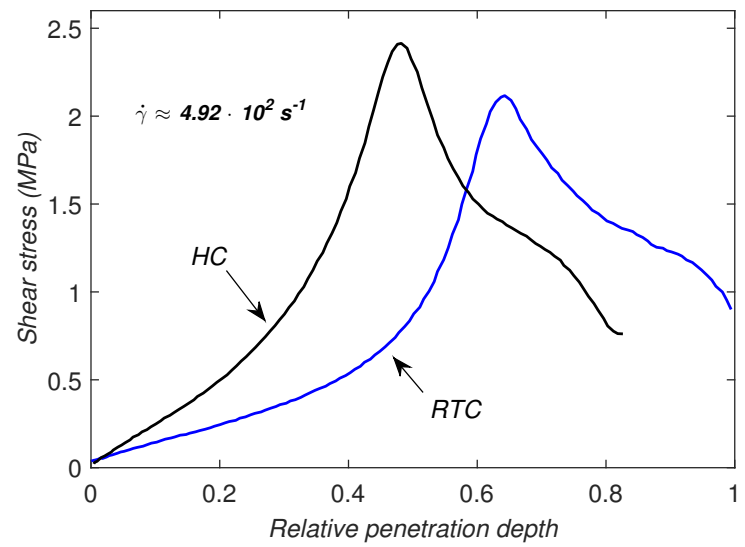

(b)

Figure 48. Shear results for neat PDMS demonstrating effect of cure temperature at two punch velocities: (a) $V_{p}=1.3 \mathrm{~mm} / \mathrm{min}$ and (b) $V_{p}=3000 \mathrm{~mm} / \mathrm{min}$.

\subsection{Influence of Particle Volume Fraction on PDMS Composites}

\subsubsection{Tensile Testing}

Characterization of particle-filled elastomers began by determining the effect of filler volume fraction on the material response. The tensile stress-strain curves for $\mathrm{Al}_{2} \mathrm{O}_{3}-\mathrm{PDMS}$ (HC) composites at 4.7 and 33.3 vol.\% are found in Fig. 49 along with the neat PDMS baseline response. The Young's modulus of PDMS was drastically enhanced by particle integration,

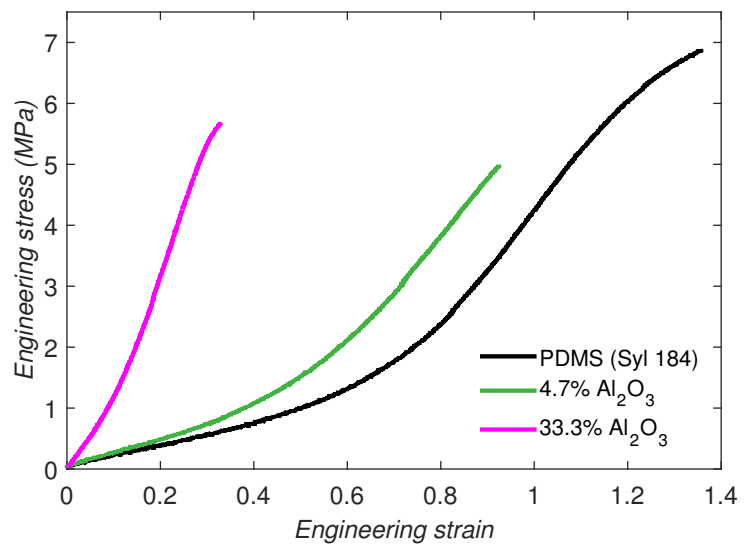

Figure 49. Tensile results showing the effect of particle volume fraction for $\mathrm{Al}_{2} \mathrm{O}_{3}-\mathrm{PDMS}$ $(\mathrm{HC})-\dot{\epsilon}=8.18 \cdot 10^{-3} s^{-1}$.

which is consistent with the rule of mixtures and other particulate theories found in Section 2.2.2. Although the linear elastic regime was maintained at low strains, an earlier transition 
to a non-linear strain hardening response occurred with increasing particle content. The $\epsilon_{u t s}$ of the systems decreased continuously with volume fraction. The ultimate tensile strength decreased (relative to neat PDMS) at both volume fractions investigated. The relative strength between composite and matrix $\left(\sigma_{c} / \sigma_{m}\right)$ was plotted against volume fraction in Fig. 50 in order to compare the results against lower bound (Eq. 7) and upper bound $\left(\sigma_{c} / \sigma_{m}\right.$ $=1$ ) models $[72,73]$. The lower relative strength values indicate weak to intermediate particle-matrix adhesion resulting in void formation at the interfaces (debonding) since the ceramic fillers are not expected to fracture at such low stresses. Upon separation at the particle interface, unstable crack propagation was expected to dominate at low particle concentrations, causing catastrophic fracture. The relative tensile strength decreased below the lower bound model at 4.7 vol.\%, whereas the response of the 33.3 vol.\% composite laid comfortably between both limits. This may be attributed to the large amount of particles acting as barriers to crack propagation.

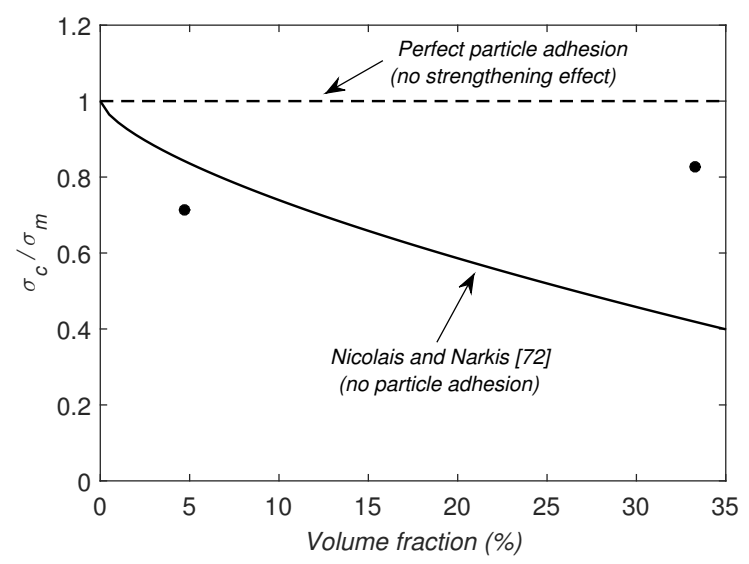

Figure 50. Relative tensile strength versus particle volume fraction for $\mathrm{Al}_{2} \mathrm{O}_{3}-\mathrm{PDMS}$ composites.

The fracture toughness of the PDMS matrix is assumed to be of large importance for composite strength. This material property describes the critical crack (or flaw) size required to cause unstable crack growth for a given applied force. Since voids nucleate at particlematrix interfaces due to debonding, it can be expected that particle size is also an important factor relating to ultimate tensile strength. Debonded particles act as inherent flaws within 
the matrix, having a cavity size equal to that of the particle [68]. Therefore, larger particles would trigger unstable crack growth at a lower force. The particles used in the present thesis were all relatively large compared to those used in nanocomposites. Fracture toughness of the matrix should be as high as possible to avoid premature failure.

\subsubsection{Shear Testing}

Particle addition was found to improve the shear strength of the material at all volume fractions considered, Fig. 51. The transverse stiffness of the materials (i.e., the initial slope of the shear stress curves) increased accordingly and reached peak stress values at a relatively constant penetration depth. Passed this depth, the resistance to penetration diminished due to a combination of tensile and shear failure; these trends shall be discussed in a later section.

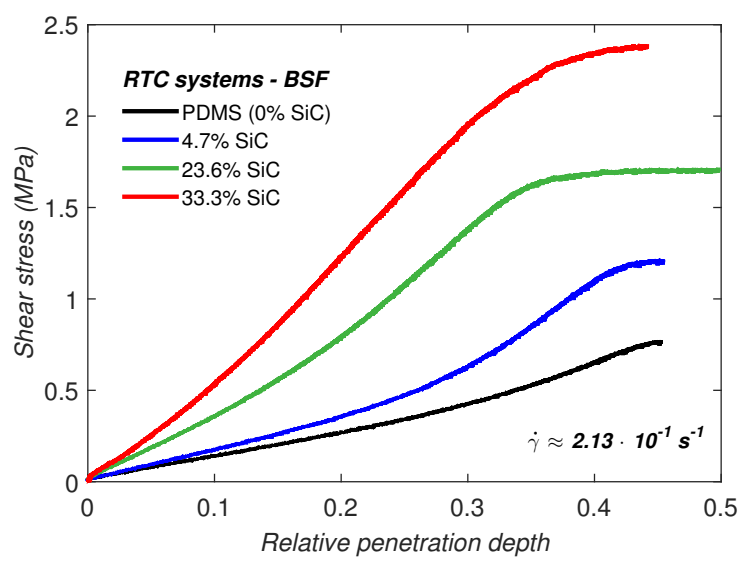

Figure 51. Shear results showing volume fraction effect for SiC-PDMS (RTC) loaded bottom-side-first.

The shear strength values were plotted against volume fraction in Fig. 52. A linear trendline was fitted to the data, although it merely serves to guide the eye. A substantial increase in shear strength at low particle concentrations was noted. Intermediate volume fractions still provided an increased strength, however at a slower rate. Another large increase in strength was obtained at higher volume fractions. Contrary to tensile testing, the shear strength of PDMS experienced substantial improvements upon particle integration. 


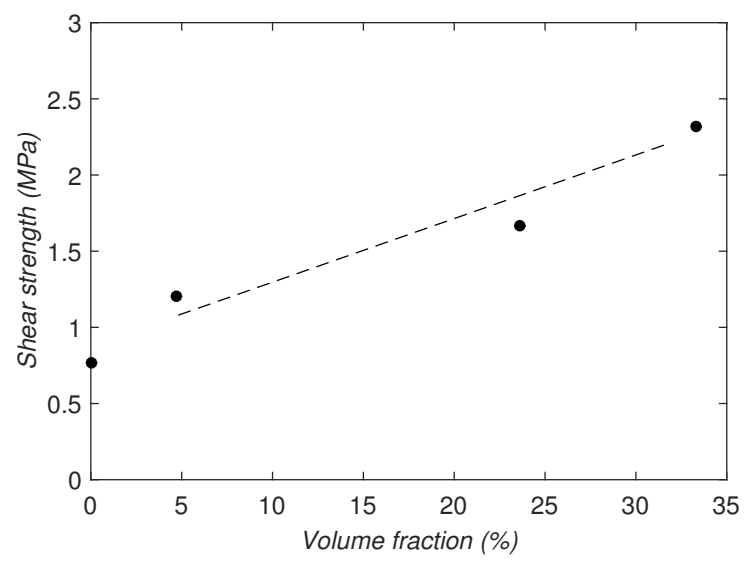

Figure 52. Shear strength of RTC composite versus particle ( $\mathrm{SiC})$ volume fraction.

\subsection{Influence of Particle Material on PDMS Composites}

The influence of particle material type on the mechanical properties of composites was investigated by varying the ceramic filler within the PDMS matrix and holding a constant volume fraction, i.e., 33.3\%. A discussion concerning particle-matrix interfacial adhesive strength and the mechanical properties of different ceramics (see Table 2) is provided.

\subsubsection{Tensile Testing}

The response of $\mathrm{Al}_{2} \mathrm{O}_{3}$ - and SiC-PDMS (HC) composites was evaluated by comparing their tensile stress-strain curves, as seen in Fig. 53. Despite similar particle sizes and shape, the SiC-PDMS exhibited substantially lower tensile strength. Its Young's modulus was also lower than that of the $\mathrm{Al}_{2} \mathrm{O}_{3}$-PDMS, even though $\mathrm{SiC}$ has higher stiffness. Based on average

component values (Table 2), the relative stiffness between both composite phases $\left(\frac{E_{f}}{E_{m}}\right)$ is approximately 198,000 and 175,000 for the $\mathrm{SiC}$ - and $\mathrm{Al}_{2} \mathrm{O}_{3}$-PDMS systems, respectively. Both ceramic particle types can be considered rigid compared to the elastomer matrix; therefore, their differences in stiffness should not greatly affect the elastic modulus of composites. The trend obtained in the present study was however opposite than what one would expect. The variations in response could have resulted from differences in particle surface chemistry since no coupling agent was used. The surface chemistry of particles is expected to influence their 
wettability and the chemical bonding strength between filler and matrix.

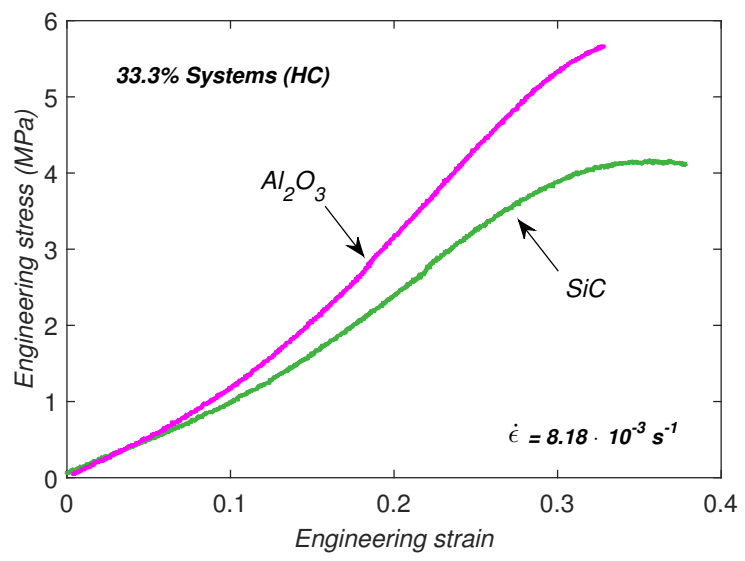

Figure 53. Tensile stress-strain curves for 33.3 vol.\% SiC- and $\mathrm{Al}_{2} \mathrm{O}_{3}$-PDMS (HC) composites, showing effect of material type $\left(\dot{\epsilon}=8.18 \cdot 10^{-3} \mathrm{~s}^{-1}\right)$.

As discussed in Section 2.2.1, modified rule of mixture equations for composite strength and elastic modulus were suggested in order to account for the adhesion of discontinuous fibers within a matrix. The equations employed efficiency factors to consider the effectiveness of load transfer between matrix and filler. Based on this logic, the results suggest that the surface chemistry of $\mathrm{Al}_{2} \mathrm{O}_{3}$ allowed for more efficient load transfer between phases as compared to $\mathrm{SiC}$. The latter could have been improperly wetted by the matrix and/or created weak interfacial bonds. The excessive yielding of the SiC-PDMS (Fig. 53) which led to a plateau prior to failure was likely due to particles debonding from the matrix, thus failing to carry additional load.

The effect of strain rate on the response of these composites was studied and compared with the results from neat PDMS (Fig. 46) in order to determine the role played by the particles. The strain-rate sensitivity analysis for both composites is shown in Fig. 54. An increase in Young's modulus was observed for both composites at higher displacement rates. On the other hand, the effect of strain rate for the neat PDMS was only noted upon the transition to a non-linear strain hardening response. As demonstrated in Fig. 49, this transition point occurred at lower strain values with increasing particle concentration. The particles may have assisted in the local alignment of polymer chain segments, therefore 
inducing early strain hardening behaviour. It is possible that strain rate effects only occur once the individual chain segments are strained, as opposed to being simply reoriented within the amorphous network. This could explain why the composite response at high strain rates deviated immediately from that found at low rates. Another explanation for this response could arise from inertial effects within the denser composite that are beginning to take place. An increase in tensile strength and $\epsilon_{u t s}$ was also noted at higher strain rates. A similar response was observed for the neat PDMS (Fig. 46), suggesting that this effect originated from the matrix and not due to particle inclusion.

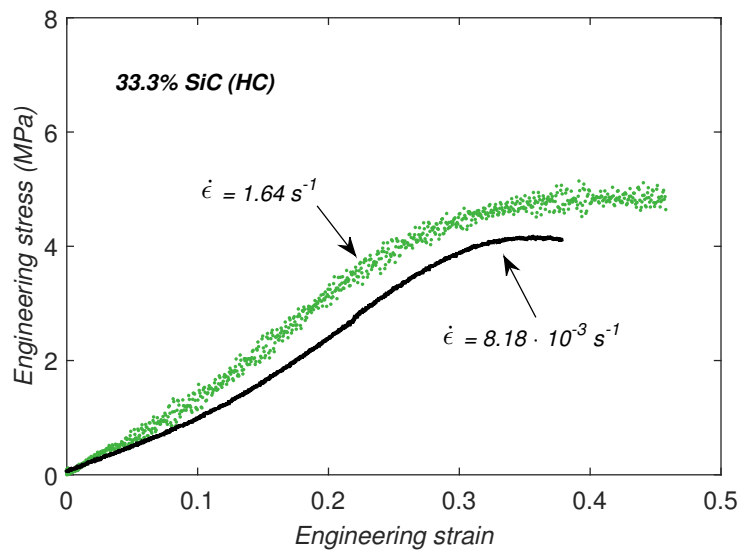

(a)

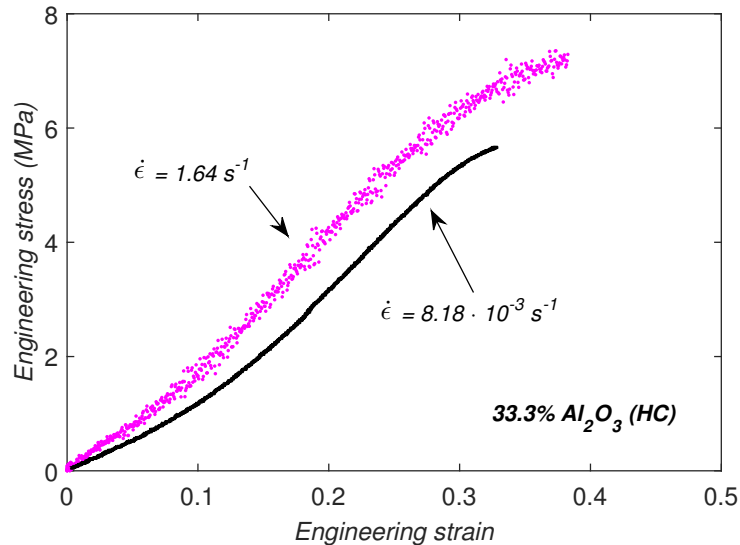

(b)

Figure 54. Tensile stress-strain curves demonstrating strain rate-sensitivity of 33.3 vol.\% particulate composites: (a) $\mathrm{SiC}$ and (b) $\mathrm{Al}_{2} \mathrm{O}_{3}$.

\subsubsection{Shear Testing}

The shear response of $33.3 \mathrm{vol} . \% \mathrm{Al}_{2} \mathrm{O}_{3}$-PDMS was assessed and compared with that of its SiC-reinforced counterpart, as seen in Fig. 55. Similar to the results from tensile testing, the $\mathrm{Al}_{2} \mathrm{O}_{3}$-PDMS composite exhibited a stiffer response along with a marked increase in shear strength. A notable decrease in shear initiation penetration depth was also observed.

Some inconsistencies in shear data were found when reversing the orientation of the disk specimens prior to loading, i.e., switching the surface in direct contact with the shear punch. Results suggested that particles were settling during the long cure process at room 


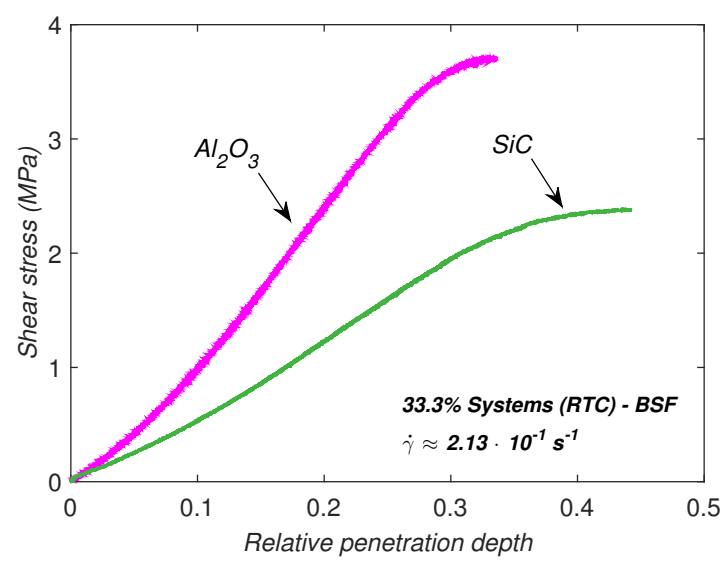

Figure 55. Shear results showing the effect of particle material types (slow rate).

temperature, which influenced the peak shear stress measurements. Distinctive features within the shear curves arose depending on whether the punch made contact with the top surface of the specimen first (TSF) or the bottom surface first (BSF). All shear data presented up to now were obtained using disks loaded in the BSF-orientation. Neat PDMS did not show any signs of loading orientation sensitivity, therefore confirming that the differences observed were caused by particle settling.

Shear results illustrating the loading rate and orientation sensitivity of 33.3 vol. $\% \mathrm{Al}_{2} \mathrm{O}_{3^{-}}$ PDMS composites are shown in Fig. 56. The response for a given orientation was highly reproducible, based on repeated experimentation. A significant difference in peak strength was observed at the slower loading rate $\left(\dot{\gamma} \approx 2.13 \cdot 10^{-1} s^{-1}\right)$. TSF-loading consistently demonstrated superior performance. When loaded in the BSF orientation, the punch came into contact with a more concentrated layer of particles that have settled directly onto this surface. This promoted earlier shear failure initiation due to particle-matrix debonding. The TSF orientation had a thin layer of matrix material separating the punch and the nearest particles, allowing for additional energy absorption prior to shear initiation. The disk orientation was found to have a negligible influence on shear behaviour at faster loading rates $\left(\dot{\gamma} \approx 4.92 \cdot 10^{2} s^{-1}\right)$. As seen in Fig. 56, the shear strength increased and converged to a singular value while failing at similar penetration depths. The transverse stiffness prior to shear failure was virtually unaffected by both loading rate and disk orientation. 


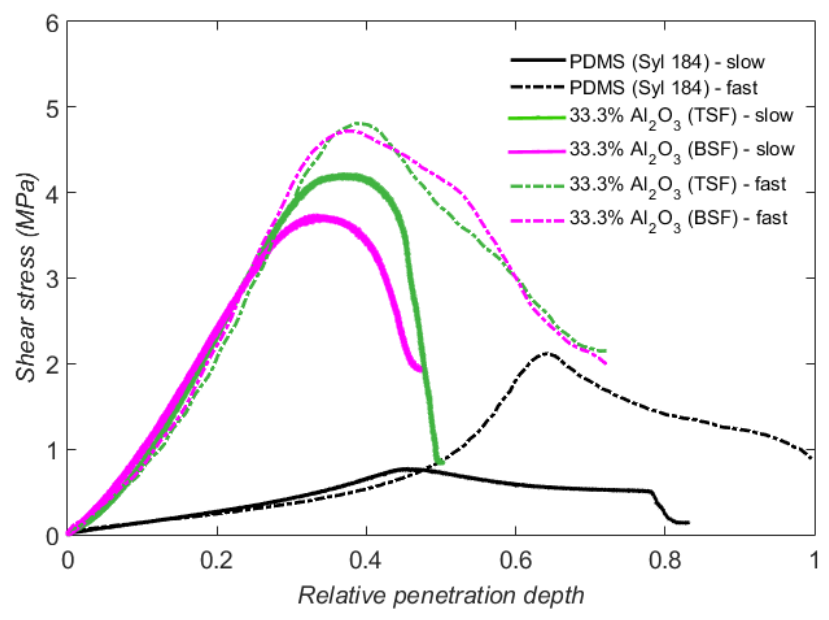

Figure 56. Shear results for $33.3 \% \mathrm{Al}_{2} \mathrm{O}_{3}$-PDMS (RTC) - strain-rate sensitivity study (slow: $\mathrm{V}_{p}=1.3 \mathrm{~mm} / \mathrm{min}$ or $\dot{\gamma} \approx 2.13 \cdot 10^{-1} \mathrm{~s}^{-1}$, fast: $\mathrm{V}_{p}=3000 \mathrm{~mm} / \mathrm{min}$ or $\dot{\gamma} \approx 4.92 \cdot 10^{2}$ $\left.s^{-1}\right)$.

A reduction of particle-settling was attempted by heat-curing the specimens, i.e., decreasing the polymerization time of the matrix. The effect of heat-curing is demonstrated in Fig. 57a. A decrease in shear strength was observed for the HC samples, demonstrating that high temperature curing negatively affected the integrity of the system when particles were introduced. This statement is supported by the results from neat PDMS in Fig. 48a, which clearly indicate an increase in both strength and stiffness upon heat-curing. High temperature curing conditions may have weakened the bonds between the particles and the matrix. On the other hand, excessive residual stresses resulting from different thermal expansion coefficients between phases could have been introduced into the matrix upon cooling.

Heat-curing of the $\mathrm{Al}_{2} \mathrm{O}_{3}$-PDMS composites did not prove to diminish the discrepancy in shear strength resulting from different disk orientations. Although it is not shown, this discrepancy along with the weakening effect (resulting from the heat cure) disappeared when increasing the punch velocity; the shear strength for both disk orientations converged to a single value, similar to Fig. 56. The effects of particle debonding seemed to be minimized upon high-speed loading. The particle suspension issue is further emphasized by noting the much larger gap in shear strength for SiC-PDMS (HC) composites loaded in both orientations, as shown in Fig. 57b. The SiC-PDMS curves for both loading orientations were highly 


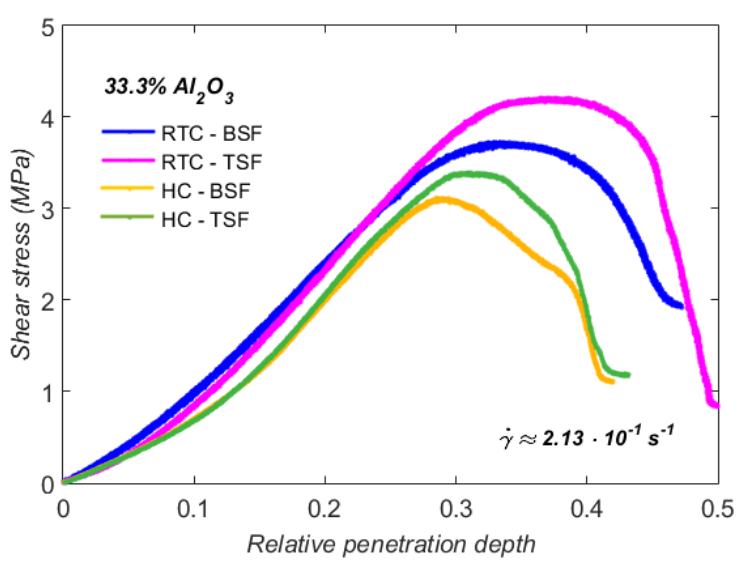

(a)

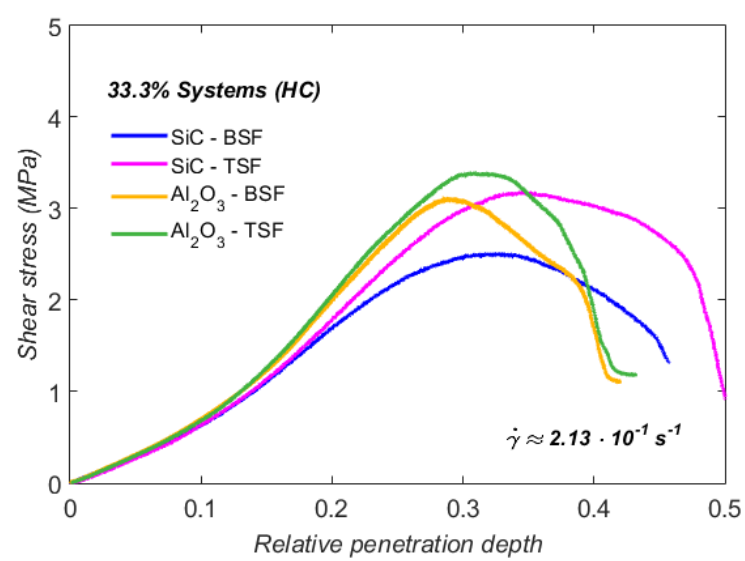

(b)

Figure 57. Shear results for (a) 33.3\% $\mathrm{Al}_{2} \mathrm{O}_{3}$-PDMS - effect of cure temperature and (b) $33.3 \%$ SiC- and $\mathrm{Al}_{2} \mathrm{O}_{3}$-PDMS (HC) composites.

reproducible. The TSF loading orientation was found to have comparable strength to that of the $\mathrm{Al}_{2} \mathrm{O}_{3}$-PDMS (HC) composite, however, a significant drop in strength was observed when reversing the sample orientation. This could be a sign of weak particle-matrix interactions, which is consistent with the results obtained from tensile testing. The transverse stiffness for both $\mathrm{HC}$ composites in Fig. $57 \mathrm{~b}$ were quite similar at low penetration depths whereas notable differences were observed for the RTC systems in Fig. 55. Contrary to the $\mathrm{Al}_{2} \mathrm{O}_{3}$-PDMS composite, the response of SiC-PDMS loaded in both orientations did not converge at faster shearing rates.

\subsection{Influence of Particle Morphology on PDMS Composites}

\subsubsection{Tensile Testing}

A morphology study was performed by comparing the results of 33.3 vol. $\% \mathrm{Al}_{2} \mathrm{O}_{3}-\mathrm{PDMS}$ (HC) samples consisting of different particle shapes, i.e., irregular and spherical. A drastic difference in response was obtained, as seen in Fig. 58. The irregular particles provided superior stiffness and tensile strength while spherical particles resulted in failure at much lower stresses, but at comparably higher strain values. The enhanced elastic modulus resulting from irregular particles could potentially be explained by the shorter interparticle distances 
(lower maximum packing fraction) [70] and the increased strain localization at the particle edges, which cause further strain hardening within the matrix. This localization effect could also explain the decrease in strain-to-failure since the sharp edges may be initiating crack propagation within the matrix, especially if particle bonding is weak. The stiffer response may have been influenced by the reorientation of high-AR particles during elongation of the specimen. As the composite is stretched, these randomly-distributed particles would have a tendency to align themselves parallel to the direction of applied force, therefore further perturbing the surrounding matrix and increasing its elastic energy [71]. Furthermore, the orientation of particles having high $A R$ affects the failure mode at the particle-matrix interface, which may have contributed to the higher strength of composites reinforced by irregular particles. Void initiation can occur at the particle interface through a combination of two mechanisms: shear or tensile failure. The fraction of particles oriented parallel to the loading direction are considered to transfer force more efficiently and conform better to the parallel coupling (iso-strain) upper bound model, Eq. 3.

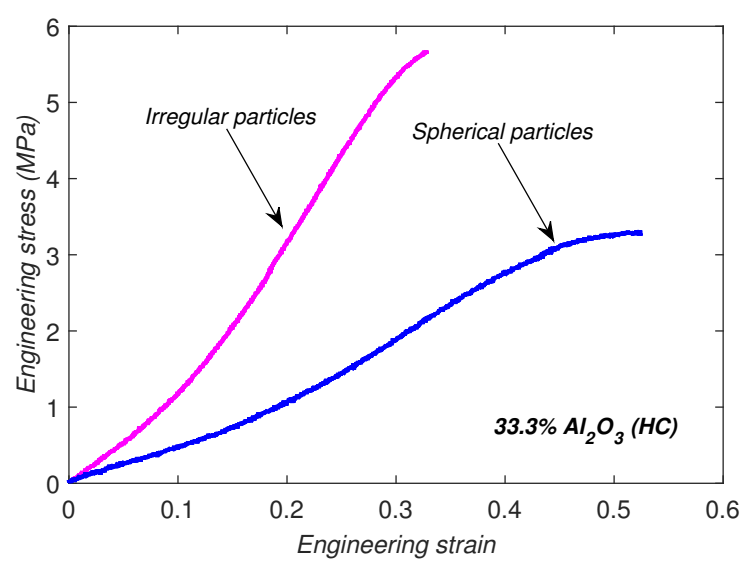

Figure 58. Tensile results showing effect of particle morphology $-\dot{\epsilon}=8.18 \cdot 10^{-3} s^{-1}$.

The driving factors for composite tensile strength are particle surface area and the particle-matrix adhesion strength [15]. Evidently, the particles must also be properly wetted by the matrix for efficient force transfer between the phases. For particles having relatively equivalent characteristic lengths, an irregular shape provides a higher surface area-to-volume ratio $(A / V)$ relative to that of a sphere. This reduces the stress exerted on the particle-matrix 
interface since the load is transferred to a larger area, thus, delaying particle interface failure. Another method of increasing $A / V$ is to decrease the mean particle size. Composites consisting of nanoparticles have been widely studied in the passed decades due to their superior mechanical response, which is associated to their increased surface area. Fu et al. [15] reviewed several studies that reported increased nanocomposite strength with decreasing particle size. Although the mean particle sizes of the $\mathrm{Al}_{2} \mathrm{O}_{3}$ powders provided by Panadyne Inc. (Table 3) were quite similar, the spherical powder consisted of many nano-sized particles (see Fig. 35e). There is however a common problem associated with the synthesis of nanocomposites: the clustering, or agglomeration of particles. For example, a localized area of nanoparticle agglomeration was noticed at the failure surface of a tensile specimen and is shown in Appendix C.1, Fig. 109. It remains unknown whether this area was responsible for material failure. Aside from this, Fig. 59a shows a relatively uniform distribution of microparticles throughout the matrix. The failure mechanism was deduced from the SEM image, showing extensive particle pullout.

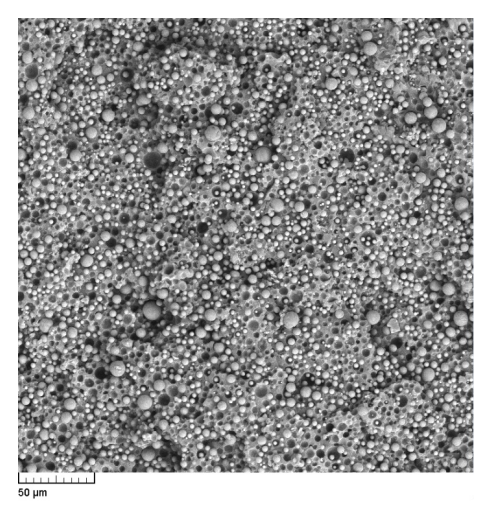

(a)

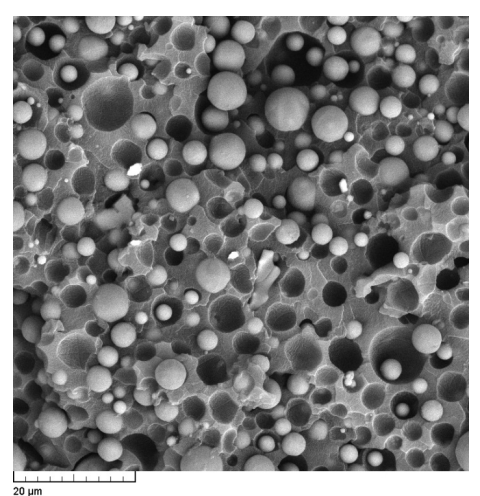

(b)

Figure 59. $33.3 \%$ spherical $\mathrm{Al}_{2} \mathrm{O}_{3}$-PDMS tensile failure surface showing particle-matrix debonding: a) 500x and b) $2 \mathrm{kx}$.

Upon further magnification of tensile failure surfaces, Fig. 59b, some small particles were noticed within the voids previously occupied by larger particles or trapped air. These particles could have clustered around larger particles during the material synthesis stage and cured in such position. It is suggested that the aggregated nanoparticles may have caused 
improper wetting, resulting in voids that likely initiated failure. The strength of particulate composites is related to fracture behaviour, therefore, its load-bearing capacity depends on the strength of the weakest path within the microstructure [15]. The potential voids seen in Fig. 59b could have likely caused the consistent reduction in composite strength although this could not be confirmed.

\subsubsection{Shear Testing}

Similar to the tensile experiments, irregular particles resulted in much higher shear strength and transverse stiffness, as seen in Fig. 60. The shear initiation penetration depth was slightly lower than for the spherical particle-reinforced system. The response of both composites was quite different once the shear strength was reached. The irregular particle-reinforced

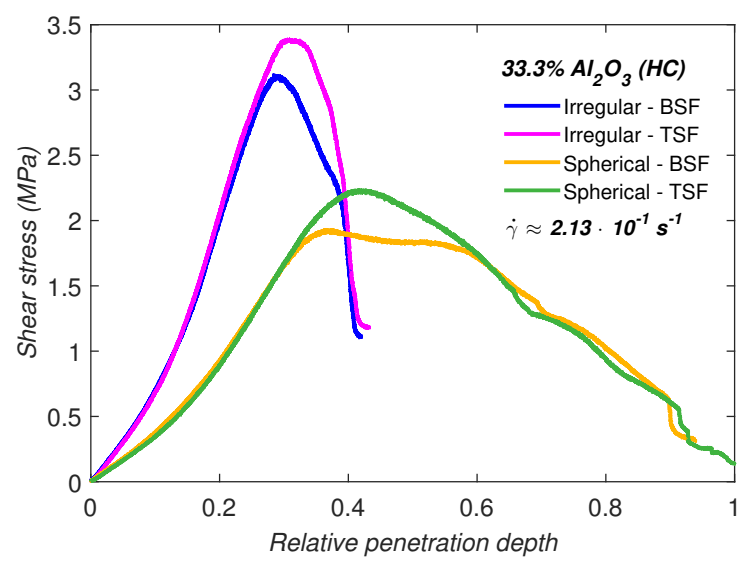

Figure 60. Shear results of $\mathrm{Al}_{2} \mathrm{O}_{3}$-PDMS (HC) composites - effect of particle morphology.

composite experienced a complete loss of strength due to cracks propagating from the bottom surface of the disk; this was a result of tensile bending stresses. Irregular particles allowed cracks to travel along the particle interface with minimal resistance due to the high stress concentrations at their angular edges. On the other hand, spherical particles blunted the crack tip, therefore denying unstable crack growth. A gradual decrease in stress occurred once shear was initiated within these composites. 


\subsection{Quasi-static Testing: Conclusions}

Prior to moving on to the analysis of ballistic penetration experiments, the main conclusions resulting from quasi-static tensile and shear testing are summarized below for quicker access. Additionally, the average tensile and shear strength values for each material have been compiled in Table 4 and 5, respectively. Although the ballistic behaviour of the particulate composites cannot be predicted merely based on their quasi-static response, the trends obtained in the present chapter may help provide a better understanding.

Table 4. Summary of tensile testing strength values along with statistical data. Two loading rates, $\dot{\epsilon}: 8.18 \cdot 10^{-3} s^{-1}$ (slow - S) and $1.64 s^{-1}$ (fast - F).

\begin{tabular}{lccc}
\hline Material/Loading rate & $\begin{array}{c}\text { Average tensile } \\
\text { strength }(\mathrm{MPa})\end{array}$ & \# trials & $\begin{array}{c}\text { Standard deviation } \\
\text { (population) }\end{array}$ \\
\hline $\mathrm{PDMS}-\mathrm{HC} / \mathrm{S}$ & 6.82 & 4 & 0.23 \\
$\mathrm{PDMS}-\mathrm{HC} / \mathrm{F}$ & 8.33 & 4 & 0.55 \\
$4.7 \% \mathrm{Al}_{2} \mathrm{O}_{3}-\mathrm{HC} / \mathrm{S}$ & 4.69 & 6 & 0.48 \\
$33.3 \% \mathrm{Al}_{2} \mathrm{O}_{3}-\mathrm{HC} / \mathrm{S}$ & 5.64 & 6 & 0.12 \\
$33.3 \% \mathrm{Al}_{2} \mathrm{O}_{3}-\mathrm{HC} / \mathrm{F}$ & 7.32 & 6 & 0.35 \\
$33.3 \% \mathrm{Al}_{2} \mathrm{O}_{3}(\mathrm{sphere})-\mathrm{HC} / \mathrm{S}$ & 3.27 & 6 & 0.02 \\
$33.3 \% \mathrm{SiC}-\mathrm{HC} / \mathrm{S}$ & 4.14 & 6 & 0.11 \\
$33.3 \% \mathrm{SiC}-\mathrm{HC} / \mathrm{F}$ & 4.74 & 6 & 0.09 \\
\hline
\end{tabular}

\subsubsection{Neat PDMS}

- All tensile dumbbell specimens were heat-cured and cut using a steel rule die to obtain proper tension data since it resulted in stiffer defect-free specimens. The curing method stiffened the PDMS response in both tensile and shear loading conditions, which was attributed to a higher degree of cross-linking between the polymer chains.

- The tensile strength of PDMS (HC) was found to increase with increasing strain rate, see Table 4. On the other hand, the tensile modulus was unaffected by variations in strain rate. Increasing the strain rate only affected the tensile curve above approximately $75 \%$ strain, resulting in additional strain hardening, higher strength, and slightly higher strain-to-failure $\epsilon_{\text {uts }}$. The average shear strength values for PDMS at 
Table 5. Summary of shear testing strength values along with statistical data. Two loading rates, $\dot{\gamma}: 2.13 \cdot 10^{-1} s^{-1}($ slow $-\mathrm{S})$ and $4.92 \cdot 10^{2} s^{-1}$ (fast - F).

\begin{tabular}{|c|c|c|c|}
\hline Material/Loading condition & $\begin{array}{l}\text { Average shear } \\
\text { strength (MPa) }\end{array}$ & \# trials & $\begin{array}{l}\text { Standard deviation } \\
\text { (population) }\end{array}$ \\
\hline PDMS-RTC/S & 0.77 & 5 & 0.01 \\
\hline PDMS-RTC/F & 2.08 & 6 & 0.10 \\
\hline PDMS-HC/S & 1.10 & 6 & 0.09 \\
\hline PDMS-HC/F & 2.62 & 6 & 0.31 \\
\hline 4.7\% SiC-RTC/BSF-S & 1.20 & 5 & 0.02 \\
\hline $23.6 \%$ SiC-RTC/BSF-S & 1.67 & 4 & 0.04 \\
\hline $33.3 \%$ SiC-RTC/BSF-S & 2.23 & 2 & 0.09 \\
\hline $33.3 \%$ SiC-RTC/TSF-S & 2.37 & 4 & 0.07 \\
\hline $33.3 \%$ SiC-HC/BSF-S & 2.50 & 3 & 0.04 \\
\hline $33.3 \%$ SiC-HC/TSF-S & 3.23 & 3 & 0.04 \\
\hline $33.3 \% \mathrm{SiC}-\mathrm{HC} / \mathrm{BSF}-\mathrm{F}$ & 4.59 & 3 & 0.18 \\
\hline $33.3 \%$ SiC-HC/TSF-F & 5.09 & 3 & 0.13 \\
\hline $33.3 \% \mathrm{Al}_{2} \mathrm{O}_{3}-\mathrm{RTC} / \mathrm{BSF}-\mathrm{S}$ & 3.73 & 4 & 0.13 \\
\hline $33.3 \% \mathrm{Al}_{2} \mathrm{O}_{3}-\mathrm{RTC} / \mathrm{TSF}-\mathrm{S}$ & 4.20 & 2 & 0.02 \\
\hline $33.3 \% \mathrm{Al}_{2} \mathrm{O}_{3}-\mathrm{RTC} / \mathrm{BSF}-\mathrm{F}$ & 4.65 & 4 & 0.19 \\
\hline $33.3 \% \mathrm{Al}_{2} \mathrm{O}_{3}-\mathrm{RTC} / \mathrm{TSF}-\mathrm{F}$ & 4.84 & 2 & 0.03 \\
\hline $33.3 \% \mathrm{Al}_{2} \mathrm{O}_{3}-\mathrm{HC} / \mathrm{BSF}-\mathrm{S}$ & 3.09 & 4 & 0.10 \\
\hline $33.3 \% \mathrm{Al}_{2} \mathrm{O}_{3}-\mathrm{HC} / \mathrm{TSF}-\mathrm{S}$ & 3.43 & 2 & 0.04 \\
\hline $33.3 \% \mathrm{Al}_{2} \mathrm{O}_{3}$ (sphere)-HC/BSF-S & 1.96 & 4 & 0.05 \\
\hline $33.3 \% \mathrm{Al}_{2} \mathrm{O}_{3}$ (sphere)-HC/TSF-S & 2.32 & 2 & 0.07 \\
\hline
\end{tabular}

both shear rates and curing conditions are found in Table 5. The transverse stiffness (i.e., slope of shear stress curves) was slightly affected by shear rate while the shear strength drastically increased at higher punch velocities and occurred at deeper penetration depths.

\subsubsection{Particle Volume Fraction Effect}

- Composite tensile strength decreased significantly when loaded at low particle concentrations, followed by a slight increase at higher volume fraction. The trends suggested that fracture mechanics played an important role. The tensile strength remained lower than that of the neat PDMS at all volume fractions, suggesting weak to intermediate particle adhesion. On the other hand, the shear strength increased at all volume frac- 
tions considered, which may indicate that adhesion strength was less critical for this loading condition.

- Both tensile and transverse modulus increased with increasing particle content. The tensile strain-to-failure decreased consistently while no clear trend was noted for the shear initiation penetration depth.

\subsubsection{Particle Material Type Effect}

- The quasi-static behaviour of particulate composites was strongly dependent on particle type. The $\mathrm{Al}_{2} \mathrm{O}_{3}$-PDMS composites consistently outperformed SiC-PDMS in both tensile and shear loading. Although the particles were of similar shape and size, the composite strength and stiffness values differed quite significantly. Interfacial adhesion strength between phases was assumed to be of prime importance for superior performance in both failure modes. Composite strength increased with strain rate for both loading modes. The tensile modulus increased with strain rate, which deviates from the behaviour of neat PDMS. It is possible that the inclusions promoted the alignment of polymer chain segments, resulting in an accelerated strain hardening effect. The transverse stiffness was however unaffected by changes in shear rate.

- Particle settling occurred during material synthesis, resulting in a non-uniform microstructure. Heat-curing the samples did not alleviate this issue. SiC particles experienced more suspension issues compared to $\mathrm{Al}_{2} \mathrm{O}_{3}$. Discrepancies in shear strength due to particle suspension issues were minimized by increasing the punch velocity.

\subsubsection{Particle Morphology Effect}

- The quasi-static response of composites was dramatically affected by particle morphology. Composites reinforced by irregular particles exhibited much higher tensile strength and modulus. Superior tensile strength was associated with particle adhe- 
sion and the orientation of irregular particles. Similar trends in shear strength and transverse stiffness were observed. Spherical particle-reinforced composites failed at significantly higher tensile strain values. On the other hand, the penetration depth for shear initiation was only marginally higher.

- The increased stiffness of composites reinforced by irregular particles was primarily associated to their larger stress concentration factors. Particles act as physical barriers that limit polymer chain motion during deformation. Those having large aspect ratios or an irregular shape perturb the matrix more than spheres [71], resulting in a strain hardening effect. Strain concentrations within the matrix occur at the particle interface and can be accentuated by corners or edges of irregular particles. The local alignment of polymer chain segments is promoted between neighbouring particles during deformation, thus, further strain-hardening the matrix. Rounded surfaces cannot provide the same level of stress and strain perturbation, consequently diminishing the stiffening effect.

- During mechanical loading, irregular particles may be required to reorient (or rotate) themselves within the system if they are not preferentially aligned with respect to the direction of material flow. The rotation of irregular particles imposes additional perturbations to the matrix, therefore increasing its complex stress state. On the other hand, spherical particles do not require reorientation due to their symmetry in all planes. Spherical particles are also more compliant to relocation in the presence of approaching particles since strain hardening near rounded surfaces is less intense.

- Once the shear strength was reached, composites reinforced by irregular particles experienced unstable crack growth due to tensile bending stresses at the bottom surface of the specimens. These composites were no longer able to provide significant resisting stress for the remainder of the penetration process. Spherical inclusions resulted in a gradual decrease in resisting stress once the shear strength was reached. As opposed 
to irregular particles, spheres provide much lower stress concentrations and can blunt the propagating crack tip, therefore denying unstable crack growth.

- SEM images showed potential voids on the failure surface of spherical particle-reinforced composites which may have resulted due to the clustering of nanoparticles onto larger micron-sized particles during material synthesis. This could have caused improper wetting and consequently, decreased performance. 


\section{Ballistic Performance Analysis: Results and Discus- sion}

The present chapter studies the dynamic response of particle-filled elastomers subjected to ballistic penetration experiments involving 16-grain steel projectiles. The objective was to assess the role of particles within an elastomeric matrix along with the corresponding failure mechanisms that came into play. Penetration experiments were performed at DRDC Valcartier to collect incident and residual velocity data of several systems. The results were then correlated to the analytical models found in Section 3. The trends observed from the quasi-static characterization of composites in Chapter 5 will serve as a means to qualitatively explain certain aspects of the material response and its failure mechanisms. The mechanical properties extracted from these experiments will not be directly employed due to a lack of data at high strain rates. For details concerning the procedure used during ballistic testing or composite preparation, the reader is referred to Section 4.

A total of 176 penetration experiments were performed in order to characterize the ballistic behaviour of the neat elastomer and several particulate composites. More specifically, the effects of particle volume fraction, material type, and particle morphology were investigated. The composites were penetrated by several different projectile geometries to elucidate the influence of penetrator nose shape on the ballistic response. The following section investigates the dynamic response of the neat PDMS matrix to penetration by all projectile types in order to develop a baseline. The subsequent sections examine variations of this response as particles having various characteristics are embedded within the elastomer. Although ballistic data was collected for four different projectile geometries, emphasis was set on the penetration of flat-nosed (RCC) projectiles. A discussion comparing the results obtained from other nose shapes (Fig. 44) is provided afterwards. The entire ballistic dataset collected for the present thesis can be found in Appendix A. Estimates of the $V_{b l}$ values for each composite are compiled in Appendix B. 


\subsection{Ballistic Response of PDMS Matrix Penetrated by Various Projectile Nose Geometries}

Prior to the analysis of particle-filled elastomers, the ballistic response of the neat PDMS matrix must be assessed for all projectile nose geometries in order to obtain a baseline for comparison. The incident and residual velocities ( $V_{i}$ and $V_{r}$, respectively) for each trial were extracted from the experimental videos while identifying and omitting shots that suffered excessive projectile yaw upon impact. The residual velocity curves for Sylgard 184 are shown in Fig. 61. The ballistic limit velocities were estimated with adequate accuracy by extrapolating the velocity data towards the horizontal axis to obtain the intercept. The results are found in Table 6. Conical projectiles suffered from significant instability during flight. Shots experiencing notable yaw were omitted, however, any yaw occurring in the orthogonal plane could not be identified. As a result, ballistic data for conical projectiles was removed from the present discussion but can be found in Appendix A.

Table 6. Estimated ballistic limit velocities $\left(\mathrm{V}_{b l}\right)$ of baseline PDMS (Syl 184) against various projectile geometries.

\begin{tabular}{l|ccc}
\hline Projectile nose & Hemisphere & RCC & Chisel \\
$V_{b l}(\mathrm{~m} / \mathrm{s})$ & 180 & 195 & 220 \\
\hline
\end{tabular}

The RCCs resulted in a higher $V_{b l}$ value compared to hemispherical projectiles (Table 6). This contradicts the results from impact studies on thin plates $[92,110]$, which demonstrates the influence of target relative thickness. As seen in Fig. 61d, the RCC projectiles experienced the most deceleration at high impact velocities. A transition seems to have occurred since chisel-nosed projectiles were found to have the highest $V_{b l}$. It should be noted that additional testing is required in order to properly assess the variability in $V_{b l}$ results.

Highly elastic materials such as ballistic gelatin are known to experience significant lateral expansion when penetrated. Consequently, improper interfacial contact between the target and the flat-nosed RCC may have occurred. Due to the elasticity of PDMS, a cavity may be forming immediately ahead of the RCC projectile nose as the target material expands 


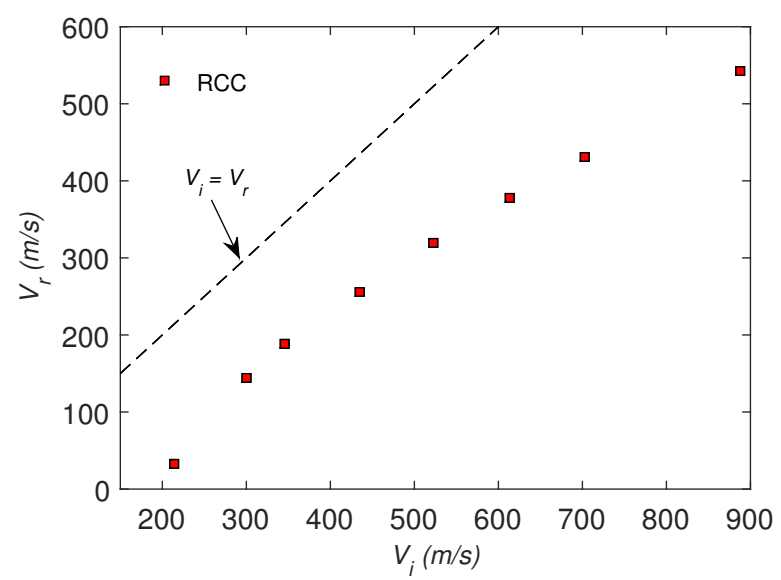

(a)

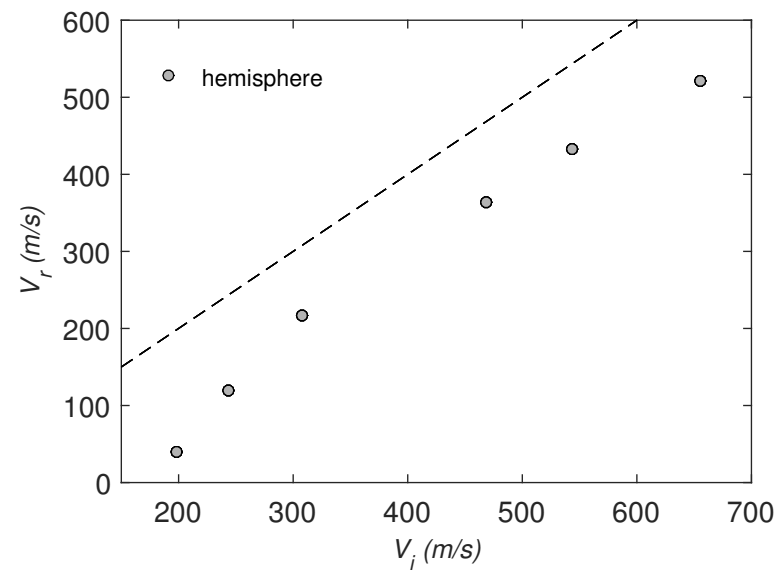

(c)

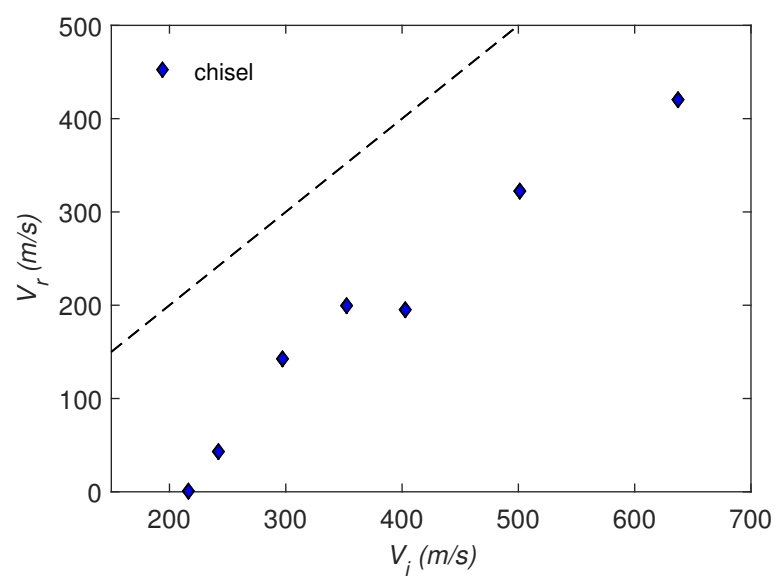

(b)

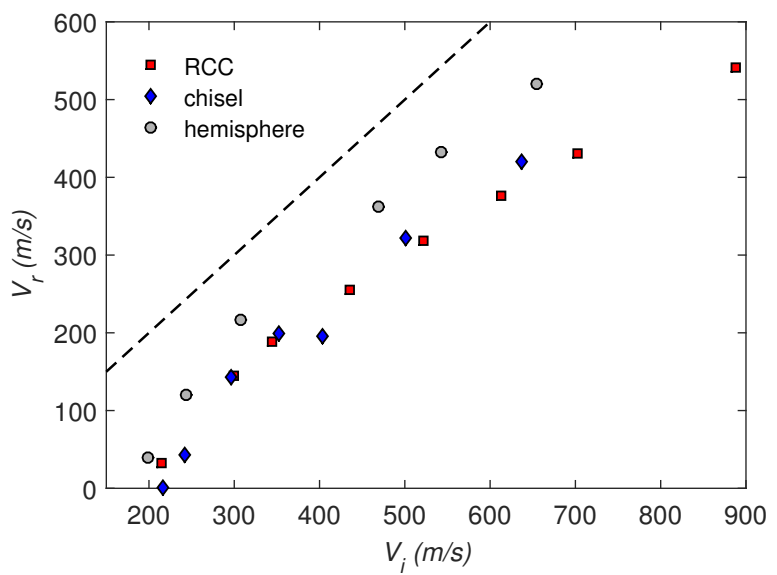

(d)

Figure 61. $V_{r}-V_{i}$ curves for neat PDMS impacted by different projectile geometries: (a) RCC, (b) chisel, (c) hemisphere, and (d) overall.

laterally during penetration. The nose may not be in complete contact with the target material, therefore localizing the resisting force to the projectile circumference. This region could then act as a sharp cutting edge and limit the resisting stress exerted by the target. The material behind this cavity would experience a tensile stress state in the lateral direction due to excessive bending. A schematic of this phenomenon is conceptualized in Fig. 62. In contrast, the smaller flat portion of the chisel-nosed projectiles would be expected to maintain better contact with the material, hence explaining why that projectile exhibited a greater $V_{b l}$. As the impact velocity is further increased, the target inertia can overcome its tendency to form a cavity so that interfacial contact is improved and nose pressure rises. 
This phenomenological explanation shall be revisited in the following section.

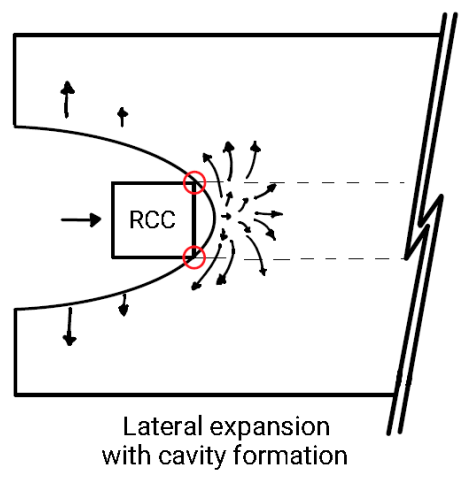

Figure 62. Schematic of lateral expansion with cavity formation ahead of RCC projectile nose. Arrows signify direction of material motion. Red circles demonstrate the diminished contact area between projectile and target.

In order to investigate the failure mechanisms associated with penetration, the velocity data has been normalized with respect to $V_{b l}$ and compared to the RI perforation and plugging models, Eq. 18 and 26, respectively. These theoretical models describe a penetration event by considering the amount of axial momentum transferred to the material ahead of the projectile nose and are illustrated in Fig. 63. For the present study, perforation shall

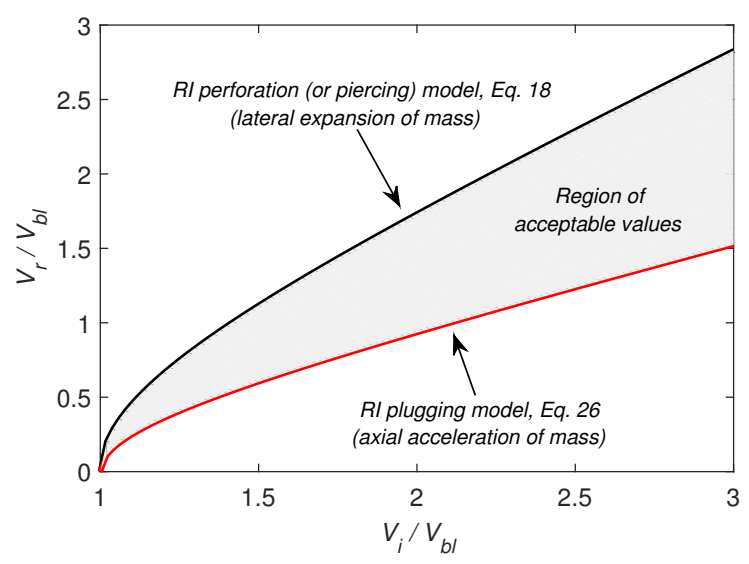

Figure 63. Theoretical RI perforation and plugging models for neat PDMS.

be defined as a piercing process where the material is simply pushed radially away from the projectile nose, resulting in no axial acceleration of the target. This behaviour is typically observed during the penetration of sharp-nosed projectiles. A plugging event is an inertia-dominated process involving the axial acceleration of material (i.e., a plug) ahead of 
the projectile nose. Such response is typically obtained by flat-nosed projectiles. The normalized velocity data of a target-projectile combination should therefore be bound between these two models.

The correlation of results obtained for neat PDMS impacted by different projectiles is shown in Fig. 64. The plugging model for hemispherical projectiles was also included (Eq. 28), which is considered to be an intermediate model since it assumes that a plug is formed (with $A_{r}=1.0$ ) but no plastic energy is absorbed upon impact [81]. The effective plugging area ratio, $A_{r}$, was set to 0.58 for chisel-nosed projectiles [44] and to 1.0 for the flat-nosed RCC. Many conclusions can be drawn from this graph. It is noted that the RCC

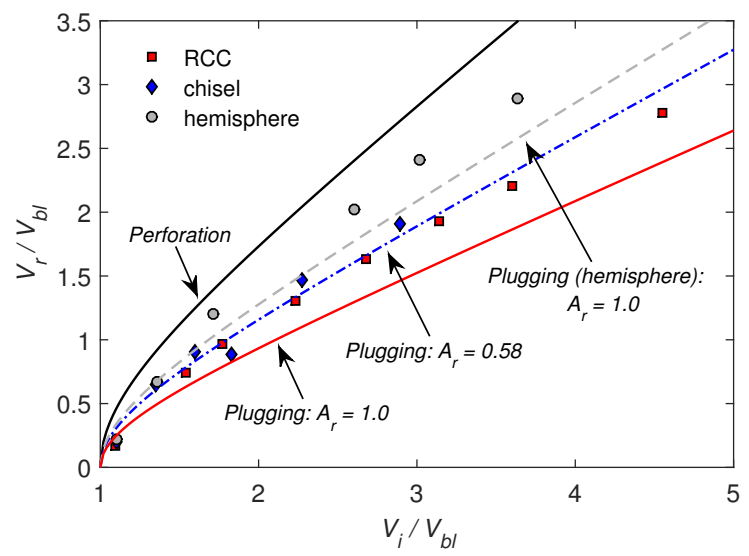

Figure 64. $\mathrm{V}_{b l}$-normalized residual velocity data of neat PDMS impacted by various projectiles along with respective RI models.

projectiles seemed to be better represented by the chisel-nosed plugging model $\left(A_{r}=0.58\right)$, suggesting that much less material was accelerated axially than predicted. This behaviour is in agreement with Fig. 62. During a penetration event, the material immediately ahead of the projectile nose can either be pushed axially along with the projectile or it can expand laterally (i.e., removing itself from the path of the projectile). Lateral cavity expansion results in less ejecta formation, which minimizes the amount of axial momentum transferred to the target. The highly elastic nature of the PDMS matrix tends to favour lateral expansion when penetrated by RCC projectiles, as confirmed by the lack of agreement with the RI plugging model in Fig. 64. The plug mass seems to have been drastically over-estimated. 
The chisel-nosed projectiles were adequately represented by their corresponding plugging model curve, although slightly under-performing. The latter may have been due to the same effect seen for the RCCs (Fig. 62), although to a much less extent. From this, one could conclude that the smaller flat region of the nose did not induce as much lateral expansion within the material. Hemispherical projectiles experienced the least resistance to penetration while their data most closely resembled the RI perforation model. This data lied directly in between the perforation curve and that of hemispherical plugging, once again suggesting less ejecta than predicted. The results from Fig. 64 show that the penetration of the PDMS targets was not an ejecta-dominated event, but was highly influenced by lateral expansion of the material due to its elastic nature and its large relative thickness. Additional evidence is provided by the post-penetration images found in Fig. 65, which show minimal variations in ejecta field for each projectile type $\left(V_{i} \approx 500 \mathrm{~m} / \mathrm{s}\right)$.

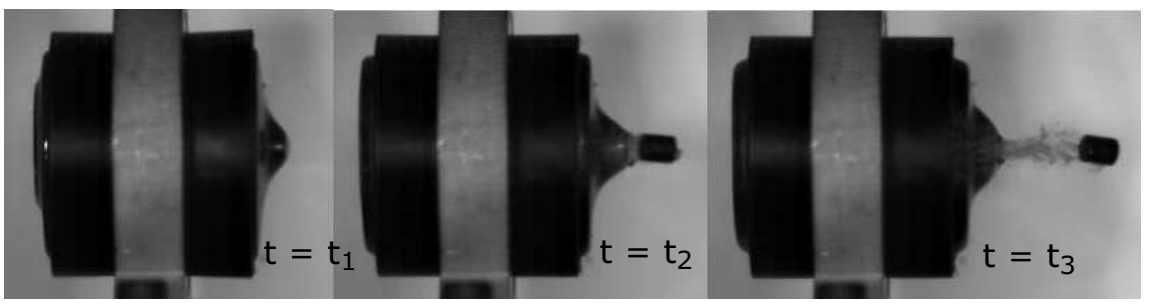

(a)

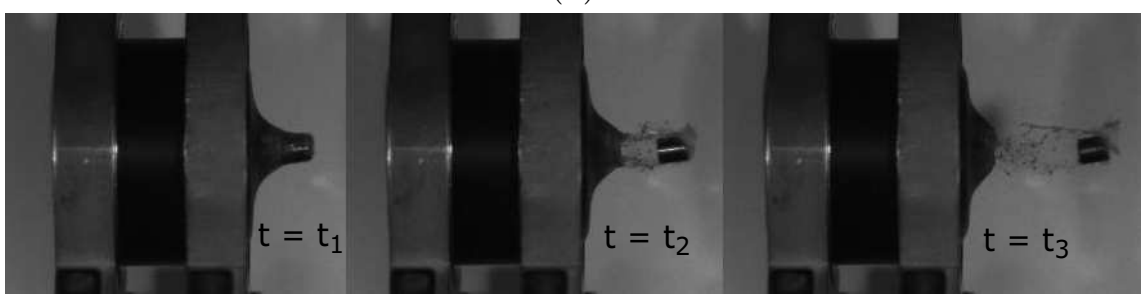

(b)

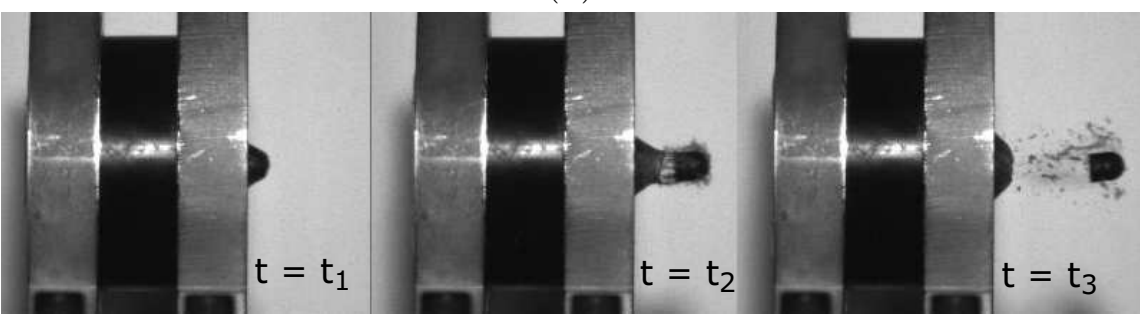

(c)

Figure 65. Post-penetration images of neat PDMS impacted by different projectiles: (a) $\operatorname{RCC}\left(V_{i}=522 \mathrm{~m} / \mathrm{s}\right),(\mathrm{b})$ chisel-nosed $\left(V_{i}=501 \mathrm{~m} / \mathrm{s}\right)$, and $(\mathrm{c})$ hemispherical $\left(V_{i}=469 \mathrm{~m} / \mathrm{s}\right)$. 
The RI models employed above account for the ballistic resistance of a target-projectile combination through $V_{b l}$, meaning that little information concerning actual material strength can be extracted; the models are primarily concerned with investigating their related failure mechanisms. The dynamic strength of the materials can be assessed using the ballistic performance parameter, $\kappa$, found in Eq. 32 [44]. This parameter assumes ideal plug formation and so it is only valid for targets impacted by RCC and chisel-nosed projectiles. The advantage of this model is that the ballistic response is normalized with respect to material density, thus allowing us to compare the performance of materials based on their dynamic strength only. Derivation of the equation along with its fundamental assumptions can be found in Section 3.3. This will prove to be useful when comparing the behaviour of various composites. The results for the neat PDMS are shown in Fig. 66.

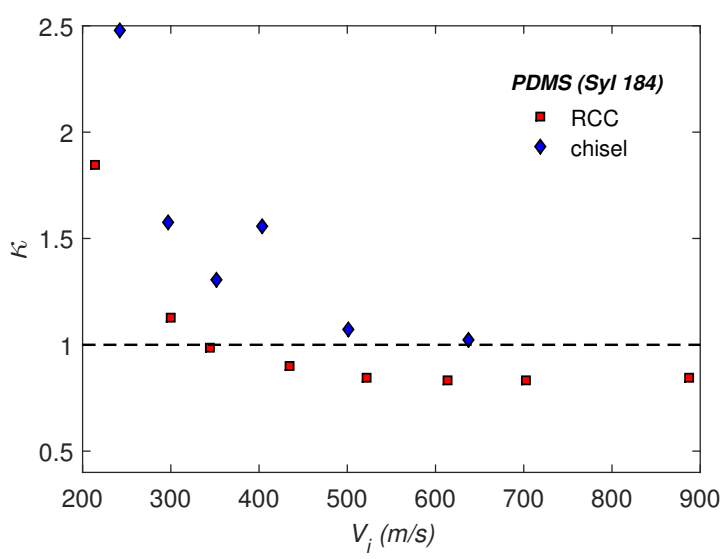

Figure 66. Normalized ballistic performance parameter results for neat PDMS impacted by RCC and chisel-nosed projectiles.

The performance of neat PDMS impacted by RCC projectiles decreased below the baseline inertial response at impact velocities greater than approximately $340 \mathrm{~m} / \mathrm{s}$. This could be expected since the performance parameter assumes that axial momentum is transferred to an ideal plug; however, it was previously noted that less material was ejected from the target and that improper contact may have occurred at the projectile nose-target interface, i.e., Fig. 62. This reduced the force acting to decelerate the projectile. On the other hand, the chisel-nosed projectile showed signs of dynamic strength over a larger range of velocities 
while converging to its inertial response at approximately $630 \mathrm{~m} / \mathrm{s}$. The superior performance suggested by this model may be implying better interface contact between projectile and target. The fact that the response converges to the hydrodynamic limit at high impact velocities is expected since good correlation was achieved with the RI plugging model (Fig. $64)$.

According to Fig. 61d, the ballistic behaviour of neat PDMS was not overly sensitive to variations in projectile nose, although hemispherical projectiles did deviate from the general trend at high impact velocities. The following sections discuss the effects of particle integration on the ballistic response of the elastomer impacted by RCC projectiles. A comparison of the composite behaviour to different projectiles is provided afterwards.

\subsection{Effect of Particle Volume Fraction}

The influence of filler volume fraction on the ballistic response of PDMS composites was investigated by varying the particle content from $0 \%$ (neat PDMS) to $33.3 \%$ by volume. Residual velocity data were collected for composites reinforced by $\mathrm{SiC}, \mathrm{B}_{4} \mathrm{C}$, and diamond particles in order to verify whether the effect of volume fraction is reproducible for different filler materials. The results for SiC-PDMS composites are presented in Fig. 67a along with their corresponding ballistic performance parameter values in Fig. 67b. Similarly, the data for composites reinforced by $\mathrm{B}_{4} \mathrm{C}$ and diamond particles are found in Fig. 68 and 69, respectively.

From these plots, it can be noticed that the addition of 4.7 vol.\% particles resulted in negligible changes in residual velocity relative to the neat PDMS. The composite stiffness received minimal enhancement at such low volume fractions and still exhibited a relatively high strain-to-failure. This behaviour was observed in the stress-strain curves resulting from

quasi-static tensile and shear experiments in Fig. 49 and 51. Lateral expansion of the material coupled with cavity formation ahead of the projectile (Fig. 62) was still assumed to be the limiting factor for improved penetration resistance. There was also a minimal 


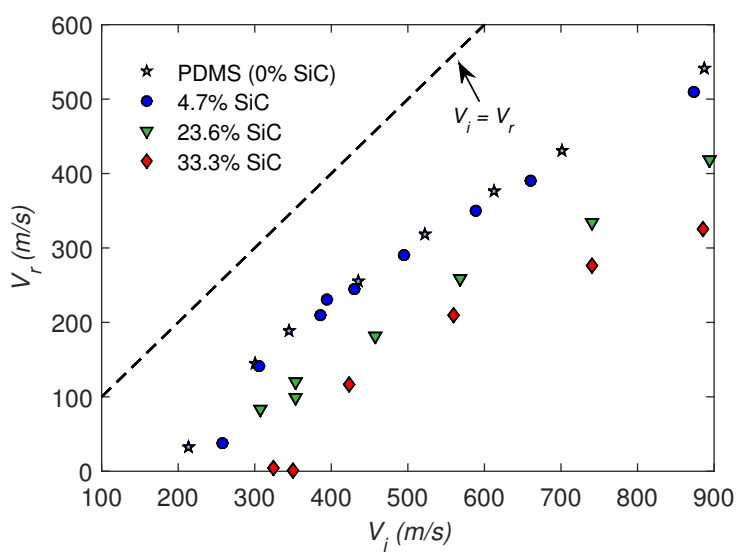

(a)

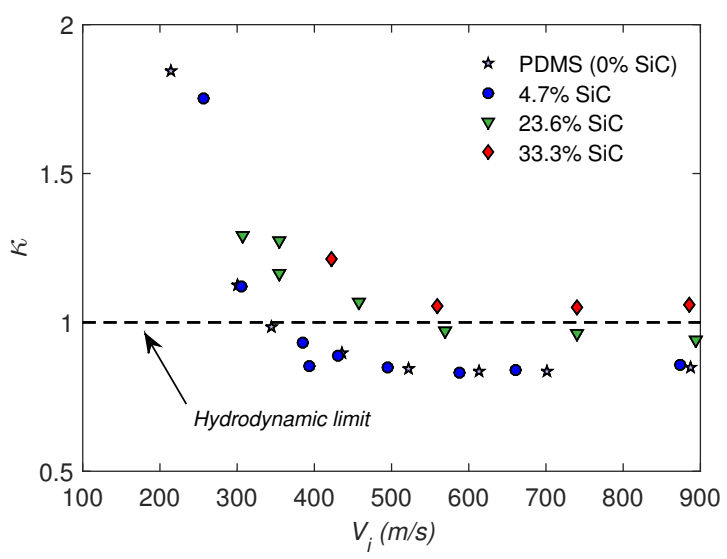

(b)

Figure 67. Volume fraction effect for SiC-PDMS composites impacted by RCC projectiles: (a) $V_{r}-V_{i}$ curves and (b) normalized performance parameter.

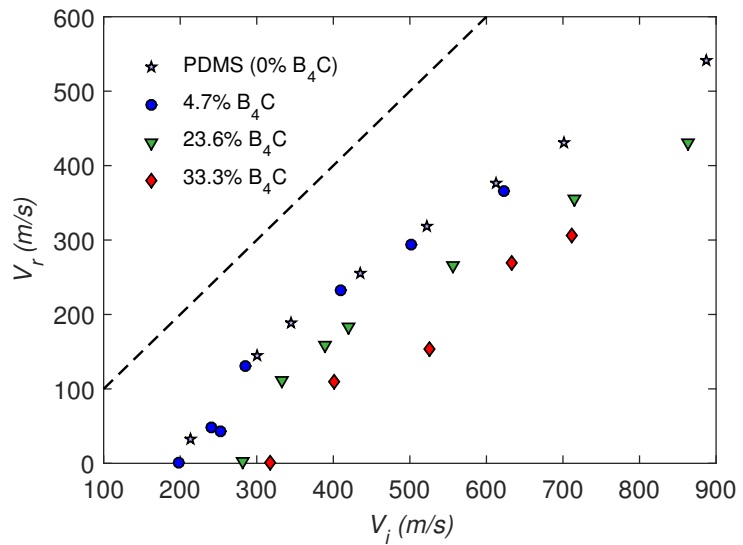

(a)

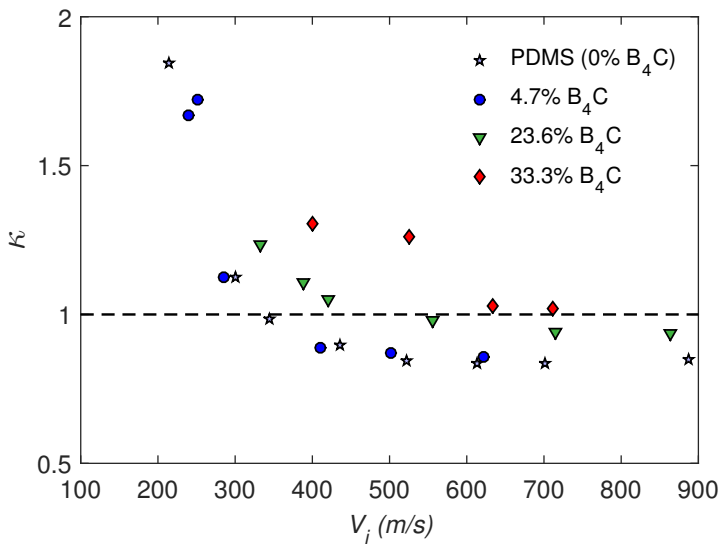

(b)

Figure 68. Volume fraction effect for $\mathrm{B}_{4} \mathrm{C}-\mathrm{PDMS}$ composites impacted by RCC projectiles: (a) $V_{r}-V_{i}$ curves and (b) normalized performance parameter.

increase in material density at such low particle concentrations, thus, negligible additional contribution from inertia. The denser diamond-PDMS composite did however see a slight change in response. As the incident velocity approached the ballistic limit of neat PDMS, an appreciable decrease in residual velocity was noticed for all 4.7 vol.\% composites. Figure 70 demonstrates that low particle concentrations contributed to relatively negligible changes in the ejecta field when impacted at velocities slightly above $V_{b l}$. The final stages of penetration at the back face showed that the 4.7 vol.\% composite nearly reached a critical point for plug formation, as seen by the plug cap formed in Fig. 70c and 70d. Particles may be promoting 


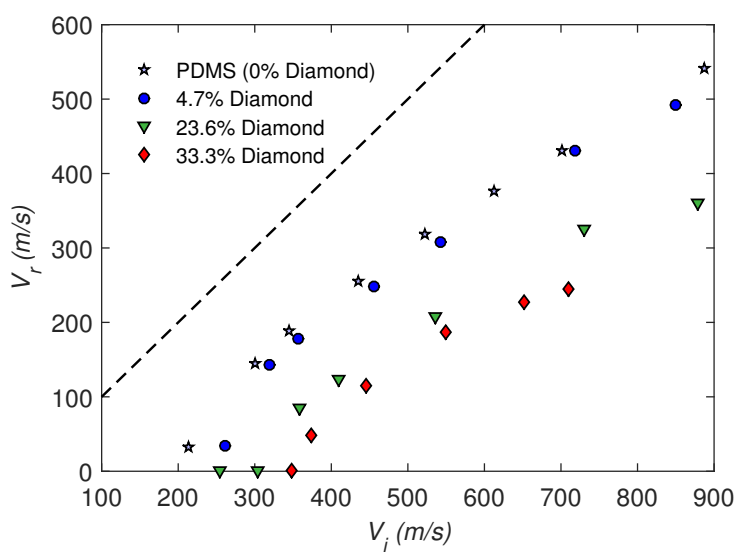

(a)

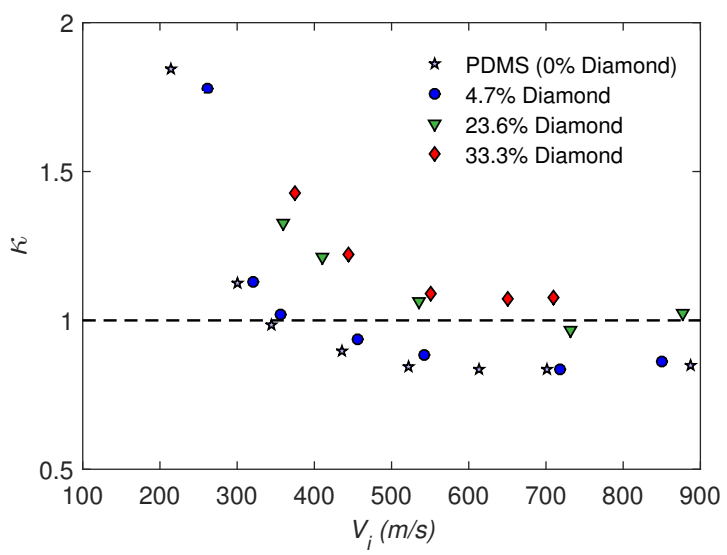

(b)

Figure 69. Volume fraction effect for Diamond-PDMS composites impacted by RCC projectiles: (a) $V_{r}-V_{i}$ curves and (b) normalized performance parameter.

crack initiation and propagation at the projectile circumference to assist in plug formation at such low residual velocities. The ejection process described above may be transferring more energy to the target than the clean tensile failure observed in Fig. 70a and 70b, therefore increasing $V_{b l}$.

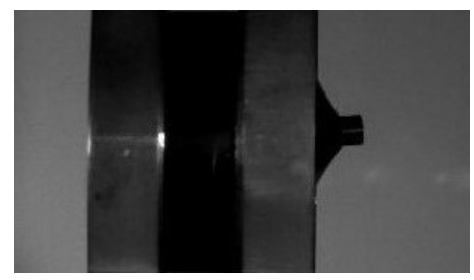

(a)

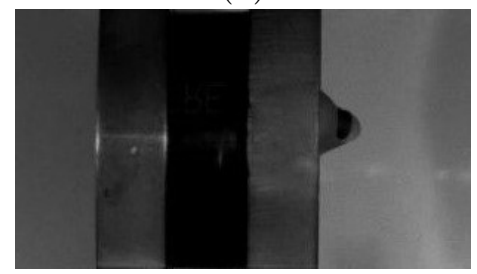

(c)

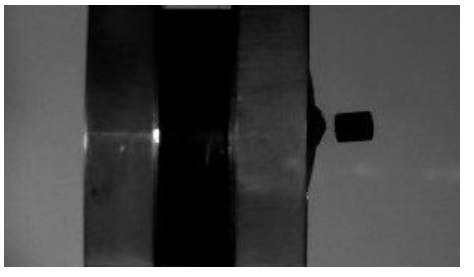

(b)

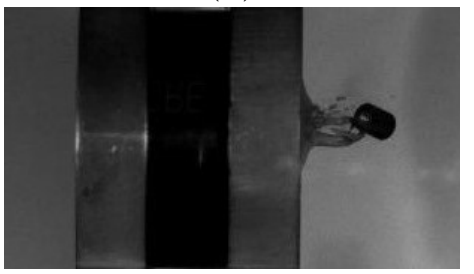

(d)

Figure 70. Post-penetration images of RCC projectiles impacting slightly above $V_{b l}$ for (a) neat PDMS at $t=t_{0}$, (b) neat PDMS at $t=t_{1}$, (c) 4.7 vol. $\%$ diamond-PDMS at $t=t_{0}$, and (d) 4.7 vol. $\%$ diamond-PDMS at $t=t_{1}$.

A marked increase in ballistic resistance was observed when the particle content was increased to 23.6 and 33.3 vol.\% (see Fig. 67a, 68a, and 69a), resulting in a higher ballistic 
limit and a lower residual velocity for a given impact velocity. The enhanced performance experienced by composites with higher particle loading fractions may be a consequence of increased resistance to lateral expansion during penetration and better interfacial contact between projectile nose and target. This assisted in the localization of shear stresses and increased axial momentum transfer. A schematic of an idealized shear plugging event is shown in Fig. 71. It is fair to assume that shear plugging became a more dominant failure mechanism with increased particle content since the elastic modulus of the composites increased significantly along with a decrease in strain-to-failure, as seen in Fig. 49. Post-penetration images of several targets shot at $V_{i} \approx 900 \mathrm{~m} / \mathrm{s}$ are shown in Fig. 72 in order to visualize the effect of particle content, i.e., the transition towards an ejecta-dominated response.

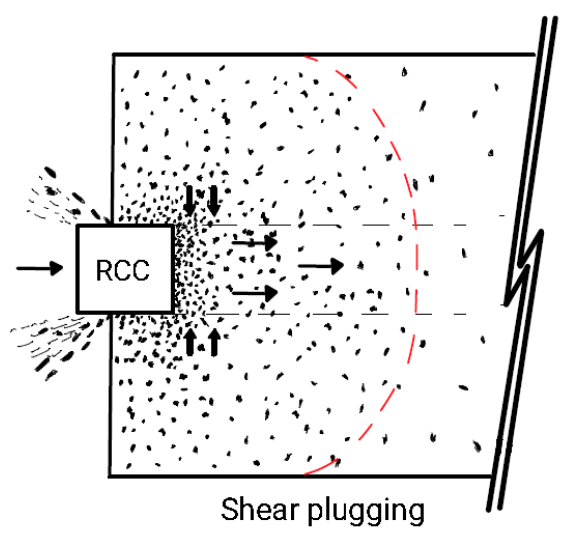

Figure 71. Schematic of idealized shear plugging event between particulate composite and RCC projectile. Arrows illustrate the restriction of lateral motion along with axial acceleration of the material ahead of projectile nose. Dashed red line shows shock wave traveling ahead of projectile and compressing material.

The residual velocity of a flat-nosed projectile that results in an ejecta-dominated penetration event is strongly influenced by target density; evidently, the increased composite density with particle addition played a role in diminishing the residual velocity. Regardless, Fig. $67 \mathrm{~b}, 68 \mathrm{~b}$, and $69 \mathrm{~b}$ indicate that the 33.3 vol.\% systems exceeded their inertial response at all impact velocities, demonstrating evidence of dynamic strength.

The effect of filler volume fraction on the $V_{b l}$ of composites is shown in Fig. 73. The numerical (estimated) $V_{b l}$ values can be found in Appendix B. It seems that the $V_{b l}$ values 


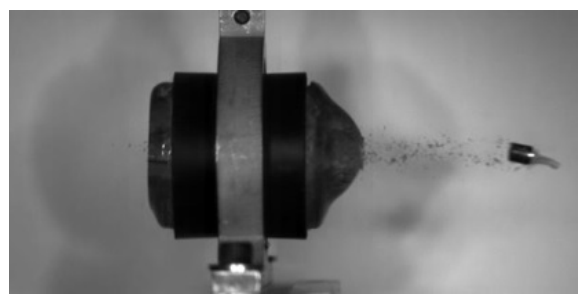

(a)

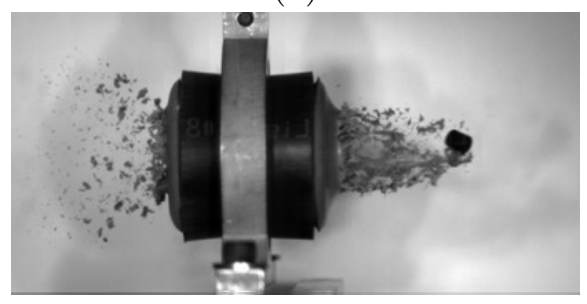

(c)

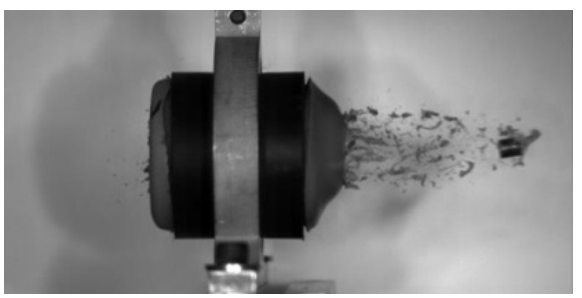

(b)

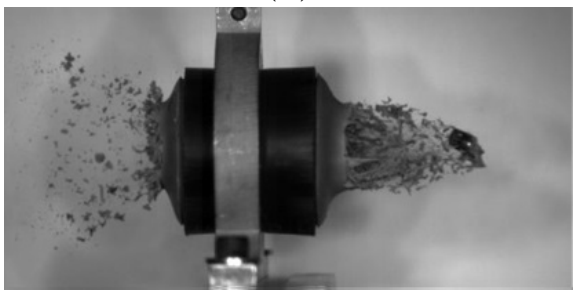

(d)

Figure 72. Post-penetration images $\left(\mathrm{V}_{i} \approx 900 \mathrm{~m} / \mathrm{s}\right)$ of SiC-PDMS at various volume fractions: (a) 0 vol.\% - neat PDMS, (b) 4.7 vol.\%, (c) 23.6 vol.\%, and (d) 33.3 vol.\%.

increased in a quasi-linear fashion, which is evident from the data of $\mathrm{B}_{4} \mathrm{C}$ and diamond composites. The SiC-PDMS did not follow the same trend, however, this could have resulted from improper estimation of $V_{b l}$, based on the available data (Fig. 67a). A trend line was merely added to guide the eye. The residual velocity data of the composites have been

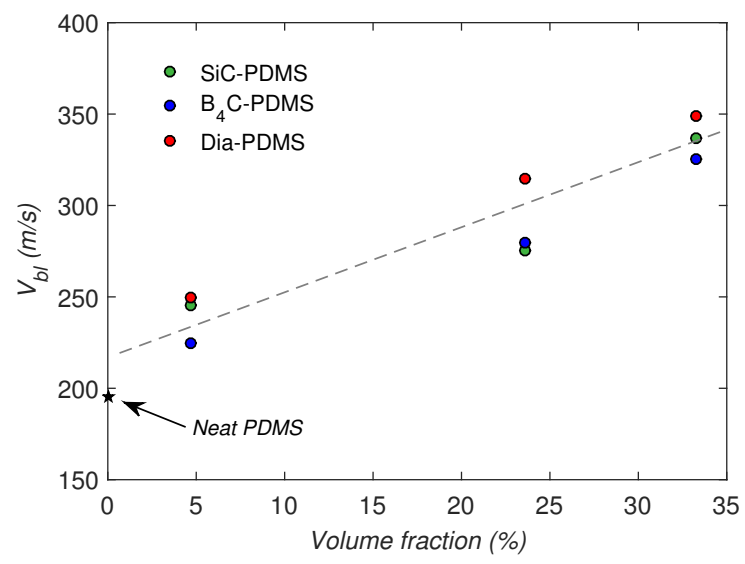

Figure 73. Estimated $V_{b l}$ values for composites having varying particle volume fractions.

normalized against $V_{b l}$ and compared to the RI perforation and shear plugging models (Eq. 18 and 26, respectively). The purpose of this was to determine whether the ejecta field observed for the particle-filled elastomers (e.g., Fig. 72) can be correlated to the response expected from a shear plugging event. Although a typical plug was not formed, the plugging 
model is expected to adequately represent the event since it assumes that the projectile accelerates a specific mass of material. The effect of particle volume fraction is further demonstrated through the normalized residual velocity curves shown in Fig. 74 .

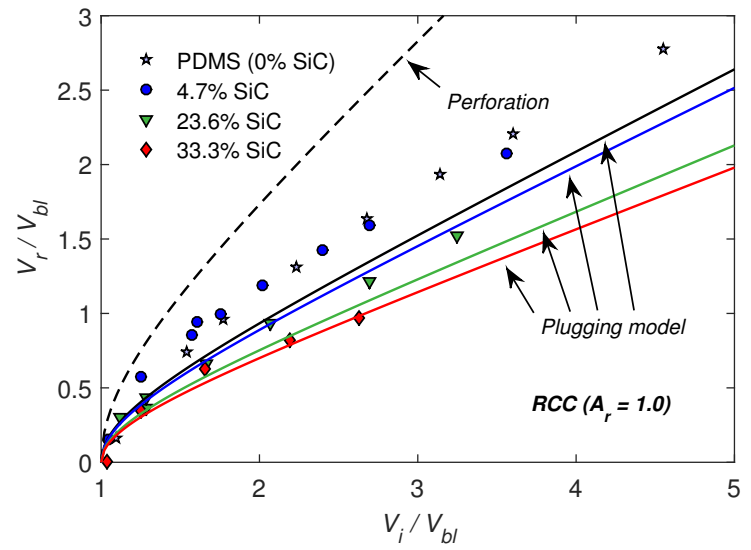

(a)

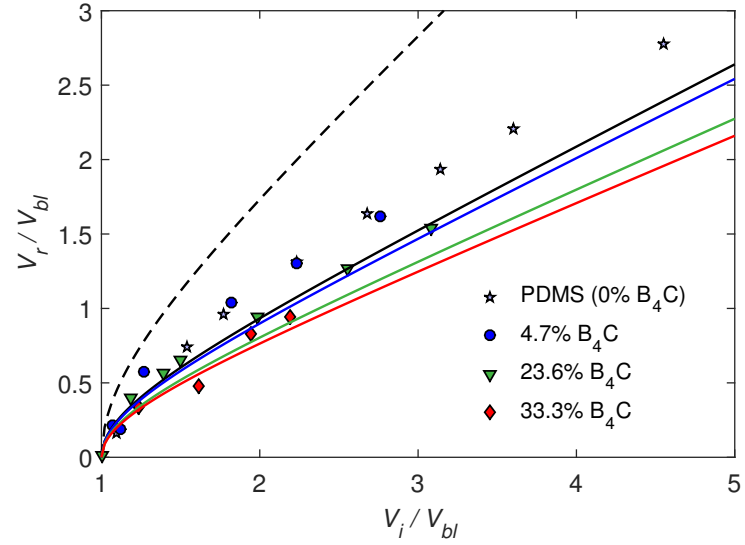

(b)

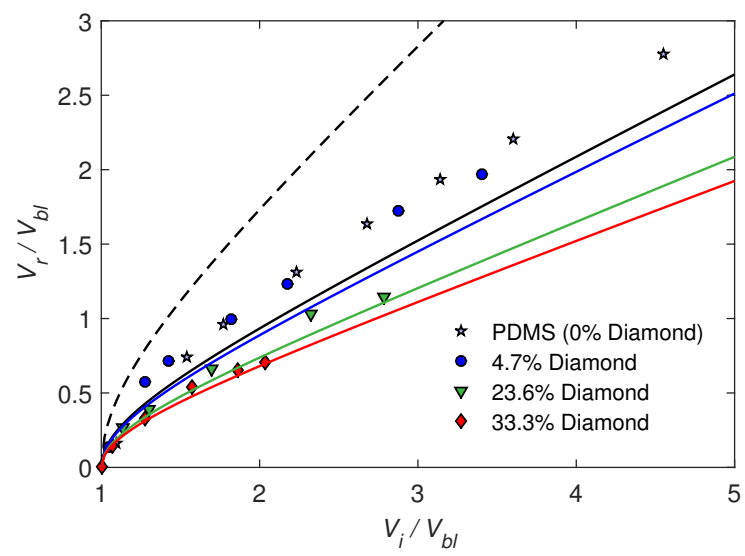

(c)

Figure 74. $\mathrm{V}_{b l}$-normalized residual velocity data with corresponding RI plugging models demonstrating volume fraction effect for: (a) SiC-, (b) $\mathrm{B}_{4} \mathrm{C}-$, and (c) diamond-PDMS composites.

The data was found to converge to their respective plugging models with increasing filler content. As previously mentioned, this was thought to be a consequence of increased resistance to lateral expansion (Fig. 71). In order to better visualize the transition towards shear plugging, a plugging fraction parameter $\left(p_{f}\right)$ is defined as,

$$
p_{f}=\frac{\left(V_{r} / V_{b l}\right)_{e x p}-\left(V_{r} / V_{b l}\right)_{p e r f}}{\left(V_{r} / V_{b l}\right)_{p l u g}-\left(V_{r} / V_{b l}\right)_{p e r f}}
$$


where $\left(V_{r} / V_{b l}\right)_{\text {exp }}$ is the normalized residual velocity obtained experimentally, while $\left(V_{r} / V_{b l}\right)_{p e r f}$ and $\left(V_{r} / V_{b l}\right)_{p l u g}$ are the corresponding theoretical values obtained from Eq. 18 and 26, respectively. The material response is expected to lie somewhere in between these theoretical performance limits. The $p_{f}$ parameter indicates the degree of correlation to the plugging response as a fraction, relative to the perforation model. It can be interpreted as the response expected when a volumetric fraction of an ideal plug is ejected along with the projectile. A $p_{f}$ value of 1 suggests an ejecta-dominated plugging event while $p_{f}=0$ indicates pure perforation. A $p_{f}$ value above unity has no physical meaning according to our model, however the physical representation of the plugging model is dependent on the definition of the projectile-plug area ratio, $A_{r}$. Alternatively, it could suggest that the plugging model does not properly represent the penetration response of the composite. Plotting data against the plugging fraction results in less clutter when comparing the response of several materials on a single graph, particularly if the data is grouped by volume fraction. Taking advantage of this fact, the $p_{f}$ values for $\mathrm{SiC}-, \mathrm{B}_{4} \mathrm{C}$-, and diamond-PDMS composites were plotted against $V_{i}$ in Fig. 75 for the three volume fractions under investigation. Although there was significant variability near $V_{b l}$, it can be clearly seen that the composites tend towards a shear plugging response $\left(p_{f}=1\right)$ as the filler content is increased beyond a certain threshold.

\subsection{Effect of Particle Material Type}

To further explore the role of particle inclusions, the data can be examined at a fixed filler volume fraction of $33.3 \%$ and the particulate material varied. The residual velocity data shown in Fig. 76a for RCC projectiles suggests that the ballistic response was indeed influenced by the choice of ceramic powder. The $\mathrm{Al}_{2} \mathrm{O}_{3}$-PDMS seemed to provide superior penetration resistance, demonstrating the lowest $V_{r}$ values for a given $V_{i}$, as well as the highest $V_{b l}$. It is however unclear as to whether this was a purely density-driven effect since the ranking in performance was found to be in the order of increasing material density. To determine whether this resulted from superior dynamic strength contributions, the materials 


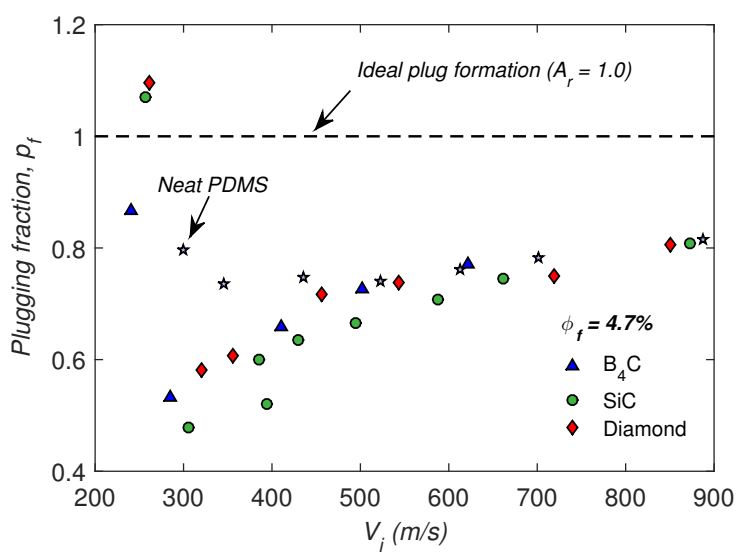

(a)

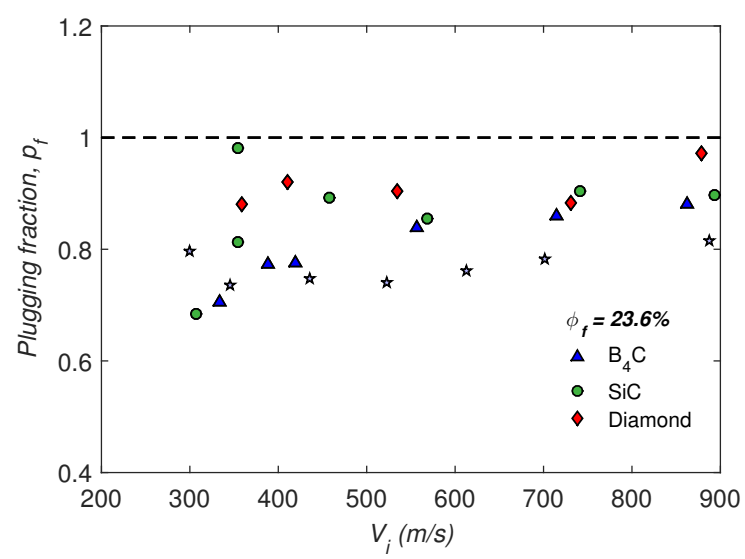

(b)

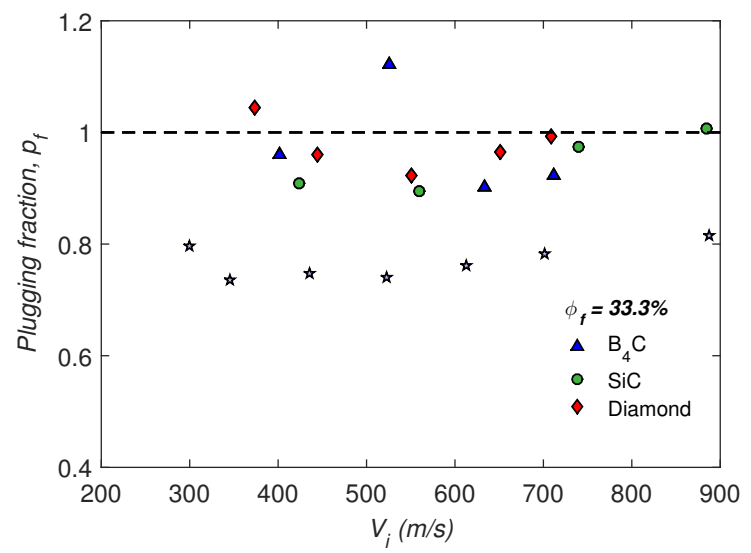

(c)

Figure 75. Plugging fraction parameter demonstrating volume fraction effect for various composites impacted by RCC projectiles: (a) $\phi_{f}=4.7 \%$, (b) $\phi_{f}=23.6 \%$, and (c) $\phi_{f}=$ $33.3 \%$.

were analyzed using the normalized performance parameter, $\kappa$. The results in Fig. 76b show that the performance ranking remained unaltered after accounting for differences in density. Although it is evident that increased material density translates to a larger amount of momentum transferred to ejecta, it is possible that density can also indirectly contribute to the work invested during penetration. This additional work can arise from lateral expansion considerations, improved contact pressure between the target and projectile, or a combination of both. It is possible that the increased inertia resulting from heavier filler material reduced the potential cavity formed ahead of the projectile nose (see Fig. 62). Better contact between the target and projectile would be promoted, resulting in higher and more uniform 
pressure distribution on the projectile nose. Compression of the target would be enhanced since the force acting on the projectile is no longer concentrated at its periphery. Proper interfacial contact is expected to increase the resisting force of the target and consequently the work required for penetration.

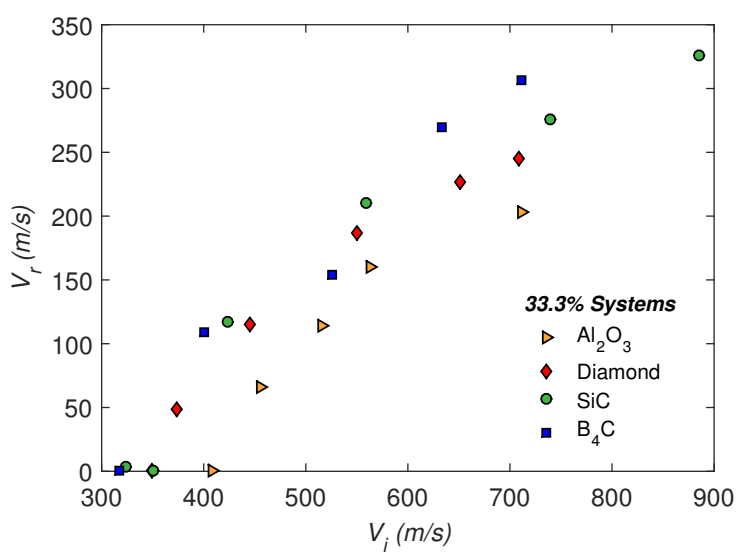

(a)

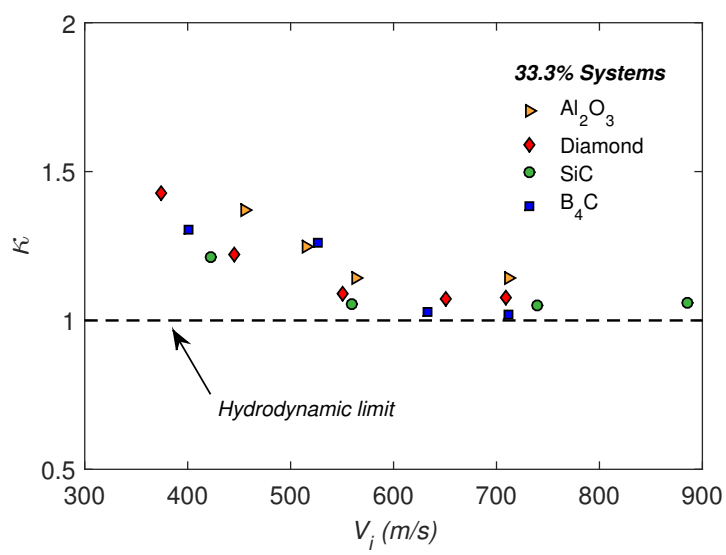

(b)

Figure 76. Effect of particle material type for 33.3 vol\% composites impacted by RCC projectiles: (a) $V_{r}-V_{i}$ curves and (b) normalized performance parameter.

The results presented above suggest that the ballistic resistance of 33.3 vol.\% particulate systems was not significantly influenced by the stiffness of the ceramic particles (see Table 2). If particle stiffness was a dominant factor for improved ballistic response, the diamond-PDMS composite would have likely outperformed the $\mathrm{Al}_{2} \mathrm{O}_{3}$-PDMS, however this was not the case. Since the ballistic response was not dependent on filler properties other than density, it can be concluded that interparticle contact (i.e., force chains) was not a dominant reinforcing mechanism. The particle content was not sufficiently high to induce a microstructural transition towards a jammed network of particles during penetration. This is in agreement with penetration experiments from Petel et al. [44] who observed that SiCEG mixtures up to 41 vol.\% did not exhibit substantial deviation from their hydrodynamic response over the range of velocities tested, which was attributed to a lack of extensive interparticle contact. It should be noted that the elastomeric systems under investigation did deviate from the hydrodynamic response at low velocities, as opposed to the EG mixtures. 
Nevertheless, the strengthening contribution primarily originated from the PDMS matrix since it is a solid capable of withstanding shear stresses, whereas the EG liquid cannot. The $\kappa$ parameters obtained from neat PDMS impacted by chisel-nosed projectiles were compared to the published values of EG [44] in Appendix C.1 (Fig. 110), which clearly illustrates the differences in response.

The ballistic limit velocities of each composite were plotted against composite density in Fig. 77. Numerical values can be found in Appendix B. The substantial deviation in performance for the $\mathrm{Al}_{2} \mathrm{O}_{3}$-PDMS at low velocities (Fig. 76 and 77) suggests that some other factor aside from material density may have contributed to these improvements. It would be interesting to investigate the influence of filler-matrix interfacial bonding strength on the ballistic behaviour of composites. No coupling agent was used to modify the surface chemistry of the particulates employed in the present study, therefore an unknown and varying degree of particle adhesion can be expected. The quasi-static results found in Fig. 53 and 55 demonstrate that the $\mathrm{Al}_{2} \mathrm{O}_{3}$-PDMS outperformed the SiC-PDMS in both strength and stiffness, which contradicts the expected trend based on particle properties only (Table 2). It is hypothesized that the carbon-based silicon carbide, boron carbide, and diamond particles may not be bonding as effectively to the matrix as the $\mathrm{Al}_{2} \mathrm{O}_{3}$ powder. A strong chemical interaction between filler and matrix may also be important during ballistic penetration.

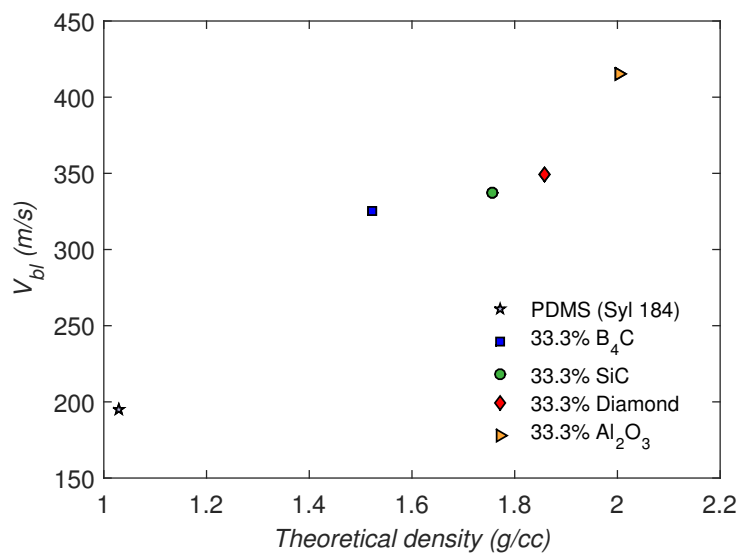

Figure 77. Ballistic limit velocity versus composite (33.3 vol.\%) density - projectile: RCC.

As discussed in Section 5.3.1, the ceramic fillers are stronger and stiffer than the PDMS 
matrix by several orders of magnitude (e.g., $\frac{E_{f}}{E_{m}} \approx 198,000$ for SiC-PDMS). As long as interparticle contact does not dominate the response, the particles can be assumed rigid. The defeat mechanisms available for such a composite are matrix cracking or filler-matrix interface failure, whereas particle breakage would only be expected if force chains were formed within the system [113]. Strong particle-matrix adhesion promotes a more uniform stress distribution within the system since the particles are capable of carrying stress without debonding from the matrix. In turn, the composite is expected to maintain its integrity, thus requiring larger forces for penetration. Poor filler adhesion could initiate premature void formation at the particle interface, resulting in a loss of composite stiffness and strength during penetration. Material failure would then be expected to occur through crack propagation, relieving the resisting stress exerted by the target. At this point, the superior performance of the $\mathrm{Al}_{2} \mathrm{O}_{3}$-PDMS composites is assumed to have resulted from higher material density and stronger interfacial bonding between the particles and PDMS matrix.

The velocity data of each composite were normalized with respect to $V_{b l}$ and compared to their corresponding plugging models, Fig. 78a. The plugging fraction for each material found in Fig. 78b shows that their ballistic response fluctuated around the shear plug model, thus, providing a decent representation for the composites at 33.3 vol.\%. It can be noticed that composites consisting of heavier particles did indeed have higher $p_{f}$ values.

\subsubsection{Consideration of Target Areal Density}

The effect of filler material type was further investigated by conducting experiments on 33.3 vol.\% particulate composites having different capsule lengths in order to maintain a constant areal density while varying the ceramic filler type. The areal density $\left(A_{d}\right)$ of a sheet or plate is described by the product of material density $(\rho)$ and its cross-sectional thickness $(t)$. For multi-layered systems, the areal density is equal to the sum of the product for each layer $(i)$, i.e., 


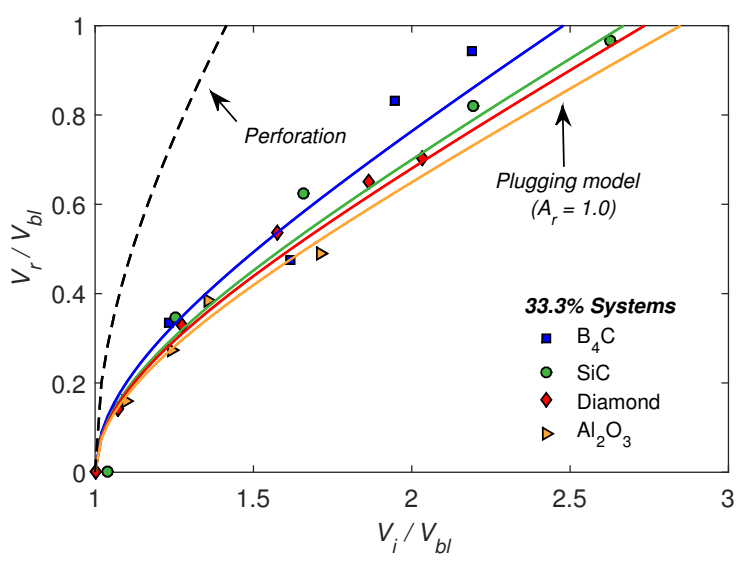

(a)

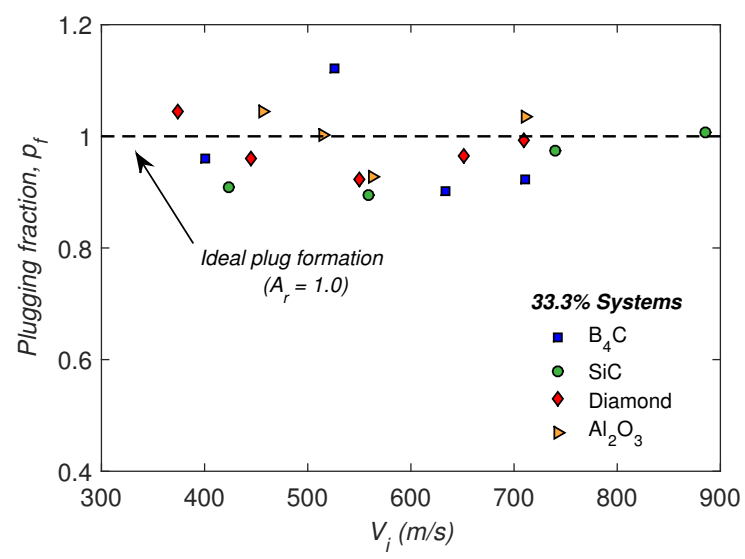

(b)

Figure 78. Plugging response for 33.3 vol.\% composites impacted by RCC projectiles: (a) $\mathrm{V}_{b l}$-normalized residual velocity data with corresponding RI plugging models and (b) plugging fraction parameter.

$$
A_{d}=\sum \rho_{i} \cdot t_{i}
$$

Targets were impacted by chisel-nosed projectiles for the present study. The results of $33.3 \%$ SiC-PDMS capsules (37.9 mm average length) were compared to composites of equivalent areal density consisting of the lightest particles, $\mathrm{B}_{4} \mathrm{C}$, having an average capsule length of $44.3 \mathrm{~mm}$, and the densest particles, $\mathrm{Al}_{2} \mathrm{O}_{3}$, with an average length of $33.4 \mathrm{~mm}$. The average areal density resulting from the capsules under consideration was $66.2 \mathrm{~kg} / \mathrm{m}^{2}$ with a standard deviation of $0.4 \mathrm{~kg} / \mathrm{m}^{2}$. The constant-areal-density analysis allows us to compare targets that have equivalent ideal plug masses, i.e., the same amount of axial momentum transferred to the target (equivalent inertial contribution). The differences in ballistic response from the targets of varying lengths should therefore be directly associated to the work required for penetration and the energy absorbed during impact. The residual velocity data for the three sets of capsules are shown in Fig. 79a.

The velocity data for $\mathrm{SiC}$ and $\mathrm{Al}_{2} \mathrm{O}_{3}$ composites were found to converge quite nicely when considering a constant areal density. A single ballistic limit velocity was obtained for the two composites although the shorter $\mathrm{Al}_{2} \mathrm{O}_{3}$-PDMS targets seemed to have a slightly 


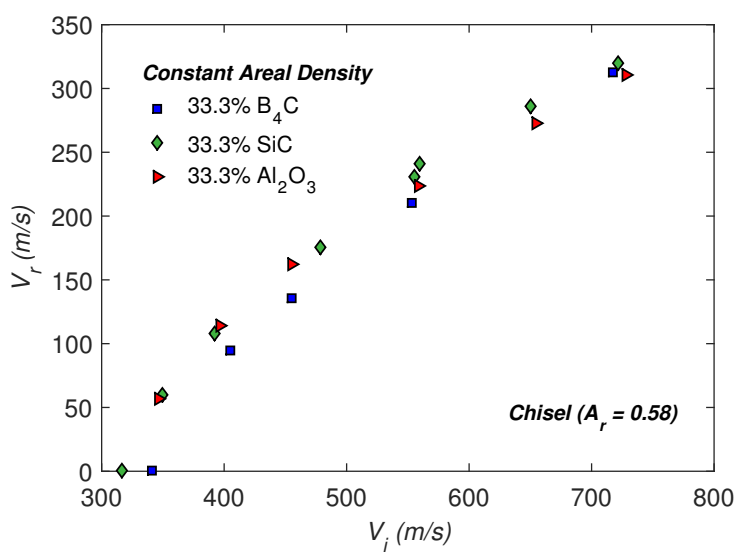

(a)

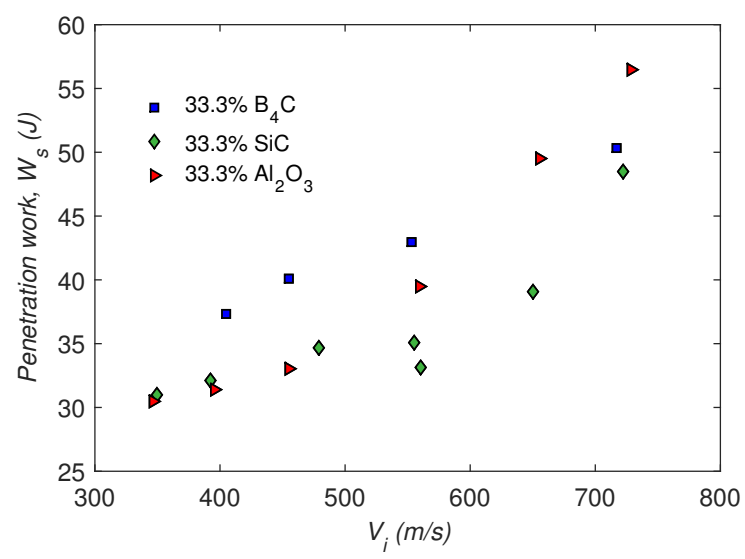

(b)

Figure 79. Constant areal density study (chisel-nosed projectiles): (a) $V_{r}-V_{i}$ curves and (b) work required for penetration.

better performance at higher impact velocities. On the other hand, the longer $\mathrm{B}_{4} \mathrm{C}-\mathrm{PDMS}$ capsules were found to perform better at low impact velocity, resulting in the highest $V_{b l}$. This response could be explained by the fact that thicker targets are capable of experiencing larger resisting stresses, reaching a theoretical maximum during penetration of semi-infinite targets [81]. Figure 21b demonstrates that the peak stress in a target increases with thickness due to a diminishing influence from the front and back faces, which act to lower the penetration resistance. Based on the same logic, it could be postulated that the thinner $\mathrm{Al}_{2} \mathrm{O}_{3}-\mathrm{PDMS}$ capsules were more affected by the free surfaces, therefore experiencing a larger reduction in peak stress. The fact that its response at low velocity was identical to the longer SiCPDMS samples could be once again indicative of superior strength. Since a constant inertial contribution from ejecta was assumed for all capsules, the differences observed can be related to the work required for penetration, Eq. 33. The results for the different capsules are shown in Fig. 79b. As expected, the penetration work was highest for the $\mathrm{B}_{4} \mathrm{C}-\mathrm{PDMS}$ at low impact velocities. Although $W_{s}$ was dependent on $V_{i}$ for all particulate systems, the $\mathrm{Al}_{2} \mathrm{O}_{3}-\mathrm{PDMS}$ composite was found to be especially sensitive to increases in impact velocity. As seen in Fig. $79 \mathrm{~b}$, the work required to penetrate $\mathrm{Al}_{2} \mathrm{O}_{3}$-PDMS surpassed that of the longer $\mathrm{B}_{4} \mathrm{C}-\mathrm{PDMS}$ capsules above $V_{i} \approx 650 \mathrm{~m} / \mathrm{s}$. 
The dynamic response of composites can be better evaluated by converting the penetration work to an approximate resistive stress value. An estimate of their average resistive shear stress $\tau_{a v}$ during penetration was obtained using Eq. 34. It should be noted that this expression is only valid for shear plugging penetration events (i.e., $p_{f} \rightarrow 1$ ). This was determined to be an adequate assumption for the 33.3 vol.\% systems under investigation, as seen in Fig. 78. Consequently, the $\tau_{a v}$ values for each composite are plotted in Fig. 80. The average resistive shear stress of $\mathrm{Al}_{2} \mathrm{O}_{3}-\mathrm{PDMS}$ targets drastically surpassed that of

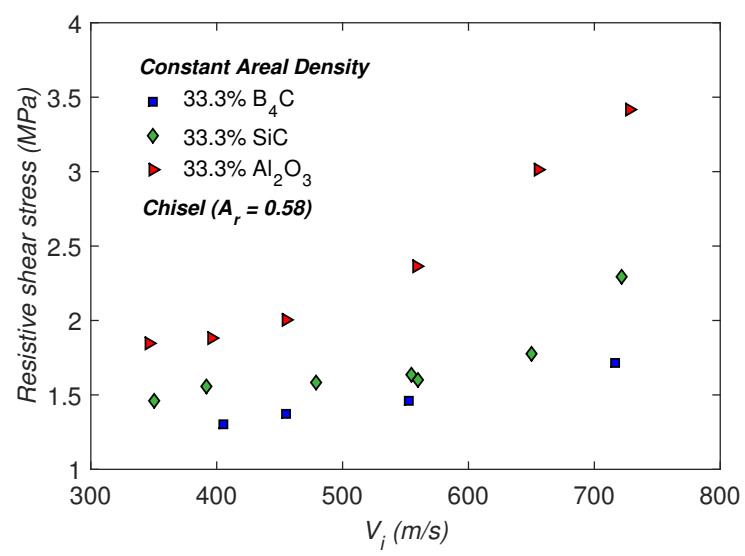

Figure 80. Constant areal density study (chisel-nosed projectiles): average resistive shear stress of 33.3 vol.\% composite targets.

the other composites with increasing impact velocity, therefore compensating for its shorter capsule length. The superior performance of $\mathrm{Al}_{2} \mathrm{O}_{3}$-PDMS was possibly a result of improved particle adhesion, which was assumed to be the cause for its relatively higher tensile and shear strength within the quasi-static regime (Fig. 53 and 55). Alternatively, the composite response could have been influenced by the different filler densities. As previously discussed, heavier particles may be promoting better contact between the projectile nose and the target due to inertial considerations. This would result in a more uniform pressure distribution onto the projectile nose, allowing for higher resisting stresses to be achieved. 


\subsection{Effect of Particle Morphology}

The influence of particle morphology on the ballistic behaviour of elastomeric composites was investigated by substituting irregular-shaped $\mathrm{Al}_{2} \mathrm{O}_{3}$ filler particles (Fig. 35d) with spherical equivalents having similar mean particle size (Fig. 35e) and comparing their response. Once again, the particle volume fraction of the composites was fixed at $33.3 \%$. The residual velocity data for the composites penetrated by RCC projectiles are shown in Fig. 81. The study suggests a strong dependence on filler morphology since the irregular-shaped

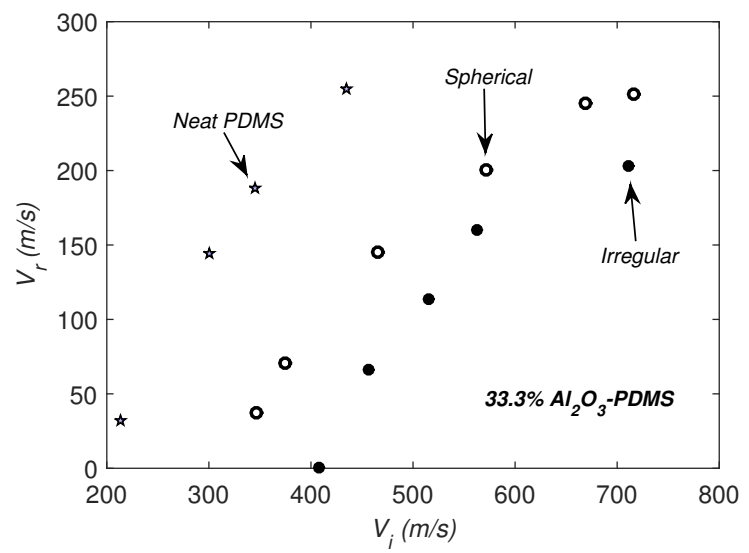

Figure 81. Particle morphology study with 33.3 vol.\% $\mathrm{Al}_{2} \mathrm{O}_{3}$-PDMS impacted by RCC projectiles: $V_{r}-V_{i}$ curves.

$\mathrm{Al}_{2} \mathrm{O}_{3}$-PDMS composite exhibited significant improvements in ballistic resistance over its spherically-shaped counterpart; a much higher $V_{b l}$ was noted. The contribution of particle morphology to the overall response stems from two different viewpoints: (1) the physical geometry effect and (2) filler-matrix interfacial adhesion considerations. Particle aspect ratio $(A R)$ may have also been an important factor to consider during dynamic loading. These shall be discussed in the subsequent sections.

The residual velocity data was normalized by $V_{b l}$ in Fig. 82a and was found to collapse onto a single curve. The only discrepancy consisted of the highest velocity data point for the irregular particulate composite, which showed an increased ballistic resistance (lower $\left.V_{r} / V_{b l}\right)$. Interestingly, a similar trend was found for spherical particulate composites at high impact velocities. The RI plugging model assumed constant work required for penetration, 


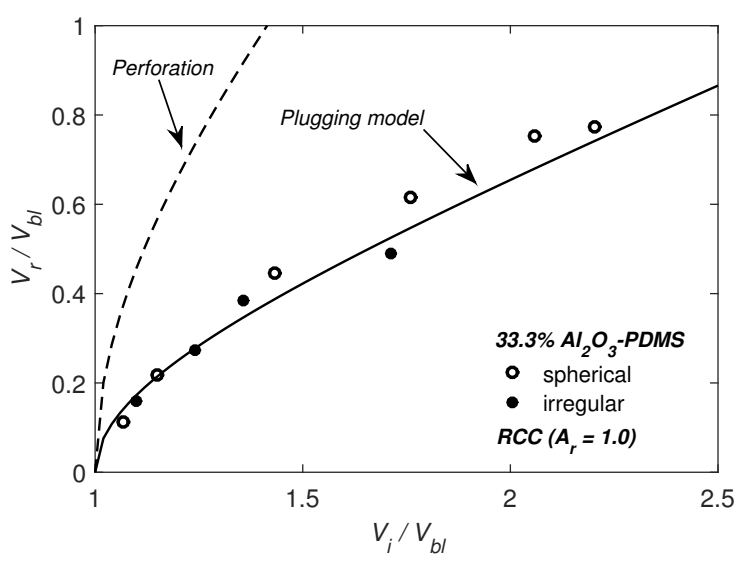

(a)

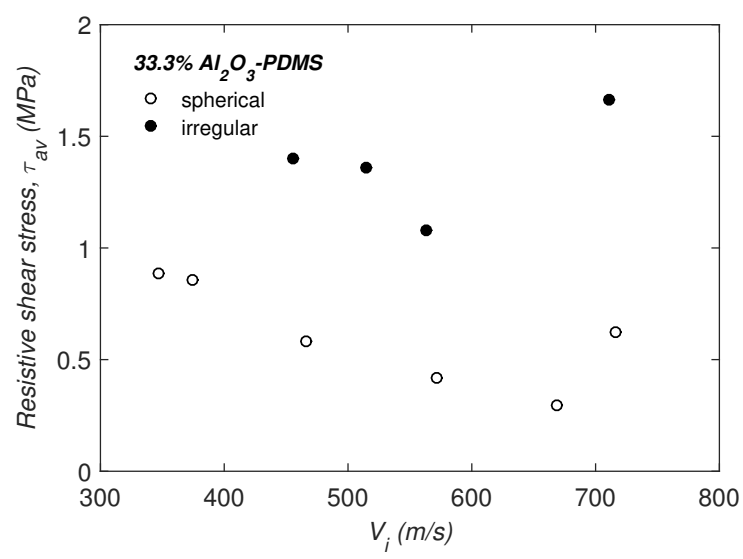

(b)

Figure 82. Particle morphology study with 33.3 vol. $\% \mathrm{Al}_{2} \mathrm{O}_{3}$-PDMS impacted by RCC projectiles: (a) $\mathrm{V}_{b l}$-normalized residual velocity curves and (b) average resistive shear stress.

which may be an acceptable assumption for metals, but possibly not so valid for particlereinforced PMCs. Assuming ideal plug formation, the average resistive shear stress $\tau_{a v}$ was calculated for both composites (Eq. 34), as shown in Fig. 82b. Although a decreasing trend was observed for $\tau_{a v}$, a sharp increase in shear stress occurred near $V_{i}=700 \mathrm{~m} / \mathrm{s}$. The effect was more pronounced for the irregular particulate composite. Such strengthening behaviour may be indicating a transition in the dynamic response of the materials and/or that target-projectile interfacial contact was improved under these conditions. In general, the RI plugging model was able to estimate the composite response relatively well, however, the sudden decrease in $V_{r} / V_{b l}$ at $V_{i} \approx 700 \mathrm{~m} / \mathrm{s}$ could not be accounted for.

\subsubsection{Effect of Particle Geometry}

The differences in residual velocity data observed in Fig. 81 were primarily attributed to filler geometry since particle surface chemistry and density were considered to be consistent for both $\mathrm{Al}_{2} \mathrm{O}_{3}$ powders. Irregular particles are believed to assist in better restricting lateral expansion of the target during penetration. This could result from large stress concentration factors at the angular edges and corners of particles that provide enhanced strain hardening of the matrix. A similar phenomenon was observed in numerical simulations of MMCs under 
dynamic compression [9]. Angular particulates were found to enhance strain hardening and demonstrated signs of increased strain-rate-sensitivity as well. Spherical particles had a less dramatic effect, which is consistent with the results from the present study. Furthermore, particles having aspect ratios above unity such as those considered here $(A R=1-10)$ perturb the surrounding polymer matrix more than spheres [71]. Reorientation of irregular particles during compression is expected to further increase the elastic energy of the system. The maximum packing fraction of these particles is also lower [70], therefore shorter interparticle distances can be expected along with increased interactions between particle stress and strain fields. Consequently, the enhanced restriction of lateral expansion provided by irregular $\mathrm{Al}_{2} \mathrm{O}_{3}$-PDMS indicates that more material is available to resist penetration and be driven axially. This provides greater pressure on the projectile nose and increases its resistive shear stress, as seen in Fig. 82b.

A different approach is now taken to further investigate the effect of particle morphology. It is tempting to correlate the enhanced ballistic response of irregular $\mathrm{Al}_{2} \mathrm{O}_{3}$-PDMS composites to the trends found upon quasi-static testing, Fig. 58 and 60 . If the improvements were indeed dominated by geometrical effects, one could expect similar trends to occur when comparing the dynamic response of spherical $\mathrm{Al}_{2} \mathrm{O}_{3}$-PDMS to a composite reinforced by different irregular particles. As an attempt to validate the latter statement, the 33 vol.\% spherical $\mathrm{Al}_{2} \mathrm{O}_{3}$-PDMS is compared to the irregular SiC-PDMS composite. The quasi-static results from Chapter 5 have been reorganized in Fig. 83. The data for irregular $\mathrm{Al}_{2} \mathrm{O}_{3}$-PDMS was included to obtain a proper comparison. As expected, the irregular $\mathrm{SiC}$ particles also provided superior stiffness and strength relative to spherical reinforcements. This assists in confirming the effect of particle geometry under quasi-static conditions. A summary of the conclusions obtained from the quasi-static morphology study is provided in Section 5.5.4.

We shall now temporarily set this discussion aside and compare the ballistic velocity data of SiC-PDMS and $\mathrm{Al}_{2} \mathrm{O}_{3}$-PDMS composites impacted by flat-nosed projectiles, Fig. 84. The corresponding $\tau_{a v}$ values have been included since the composite responses were 


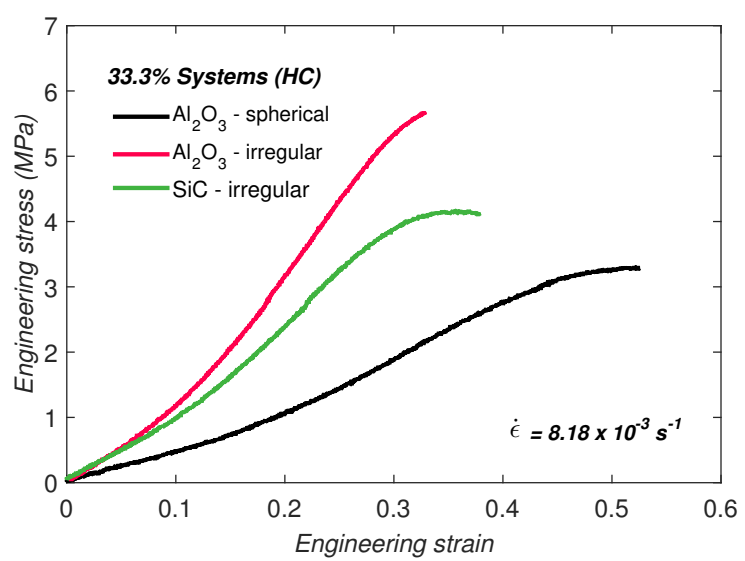

(a)

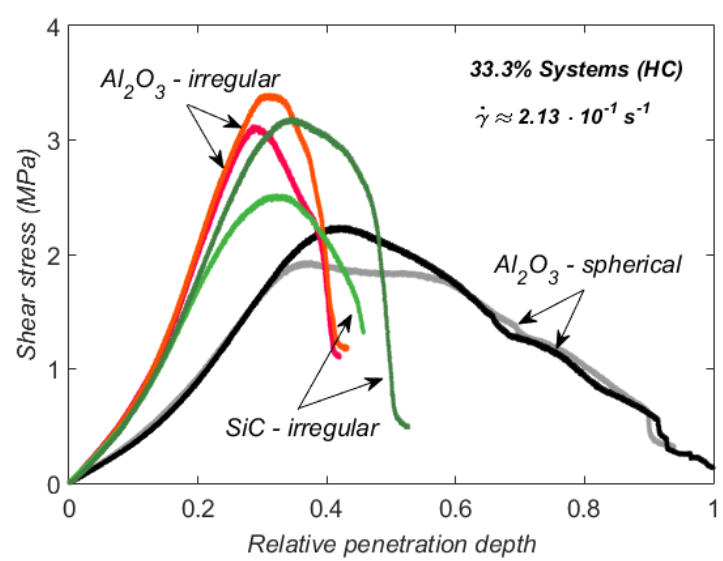

(b)

Figure 83. Comparison of 33.3 vol.\% irregular SiC-PDMS and spherical $\mathrm{Al}_{2} \mathrm{O}_{3}-\mathrm{PDMS}$ quasi-static response: (a) tension and (b) shear.

adequately described by the RI plugging model. The residual velocity data of SiC-PDMS and spherical $\mathrm{Al}_{2} \mathrm{O}_{3}$-PDMS were surprisingly similar, regardless of their differences under quasi-static conditions. The $\tau_{a v}$ values were identical for both materials when impacted by RCC projectiles, while the irregular $\mathrm{Al}_{2} \mathrm{O}_{3}$-PDMS demonstrated significantly higher resistive shear stress. The SiC-PDMS exhibited higher $\tau_{a v}$ values than spherical $\mathrm{Al}_{2} \mathrm{O}_{3}$-PDMS when impacted by chisel-nosed projectiles. Although the increase in shear stress was not drastic, this may be indicative of slightly superior performance due to particle geometry, which is consistent with Fig. 83. Once again, the irregular $\mathrm{Al}_{2} \mathrm{O}_{3}-\mathrm{PDMS}$ surpassed both other composites. In conclusion, the particle shape effect was quite significant when comparing the response of the $\mathrm{Al}_{2} \mathrm{O}_{3}$-PDMS composites, however, its influence was minimized when the irregular particle type was replaced with $\mathrm{SiC}$. This behaviour implies that some parameter was not considered in the analysis. The obvious difference between these particle types is their surface chemistry, which directly relates to the particle-matrix interfacial adhesion strength. 


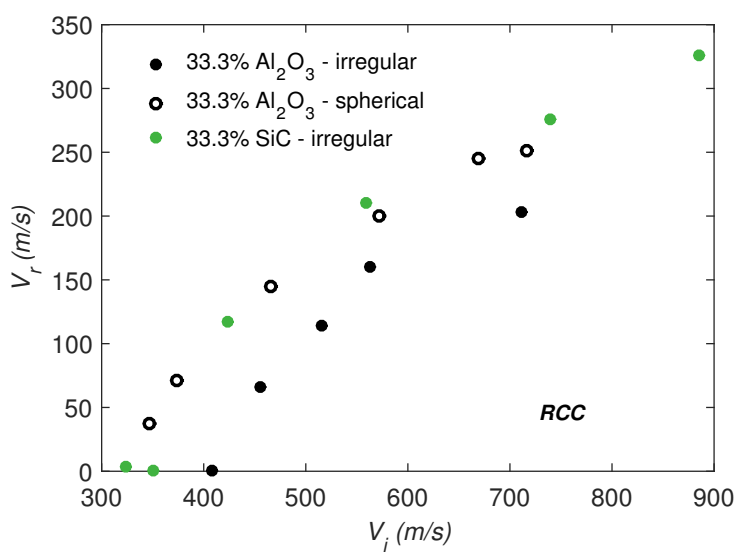

(a)

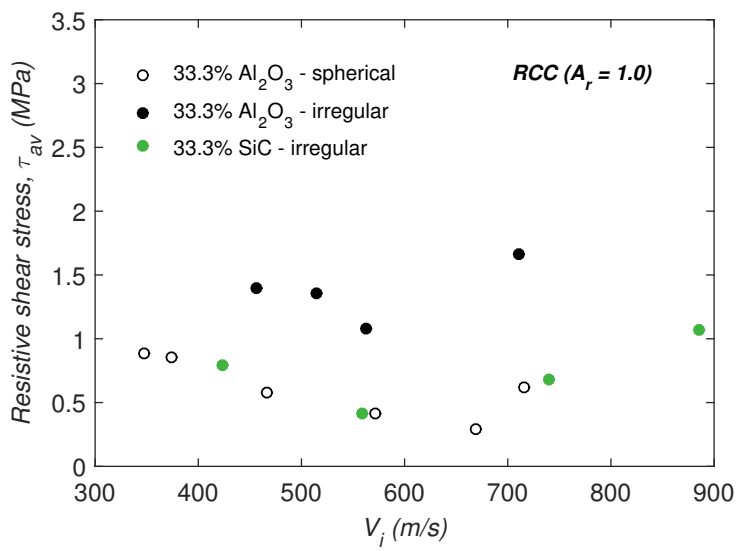

(c)

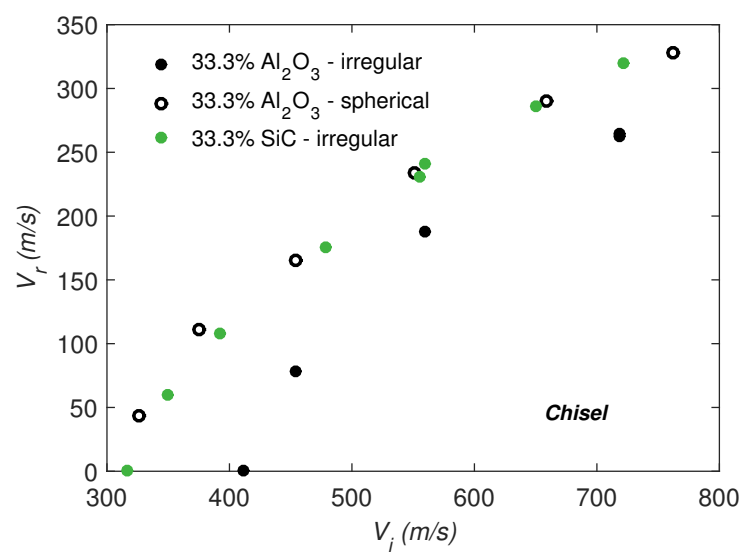

(b)

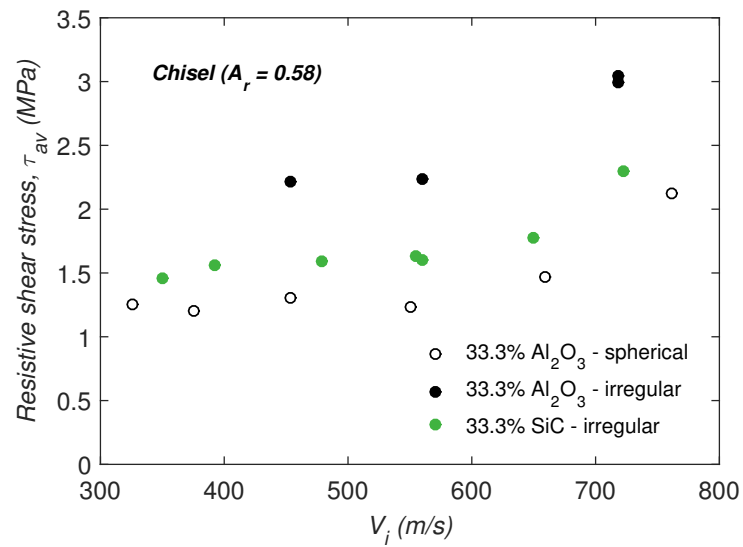

(d)

Figure 84. Comparison of ballistic results for 33.3 vol.\% irregular $\mathrm{Al}_{2} \mathrm{O}_{3}$-PDMS, SiCPDMS, and spherical $\mathrm{Al}_{2} \mathrm{O}_{3}$-PDMS composites: (a) $V_{r}-V_{i}$, RCC, (b) $V_{r}-V_{i}$, chisel-nosed, (c) $\tau_{a v}, \mathrm{RCC}$, and (d) $\tau_{a v}$, chisel-nosed.

\subsubsection{Particle Adhesion Considerations}

Since no coupling agents were used in the present study, the adhesive properties were dependent on the as-supplied surface chemistry of particles, which could be quite poor. Proper adhesion was expected to be an issue for the composites under investigation since all particles considered had relatively low $A R$. As previously mentioned, the poor interfacial adhesive strength of SiC-PDMS seemed to have been the limiting factor for efficient force transfer between phases (see Fig. 85) and was deemed the primary cause for its lower ballistic resistance as compared to irregular $\mathrm{Al}_{2} \mathrm{O}_{3}$-PDMS. Figure 84 shows that a similar ballistic response was obtained for SiC-PDMS and spherical $\mathrm{Al}_{2} \mathrm{O}_{3}$-PDMS. It is suspected that the poor adhesion 
strength provided by $\mathrm{SiC}$ particles played a strong competing effect against its favourable geometry, which may be the underlying cause for their overlapping $V_{r}-V_{i}$ curves. Therefore, proper adhesion may be required to obtain an enhanced contribution from irregular particles in terms of superior ballistic resistance. This would result in a higher and more uniform stress distribution within the composite, thus increasing the force required to penetrate the material.

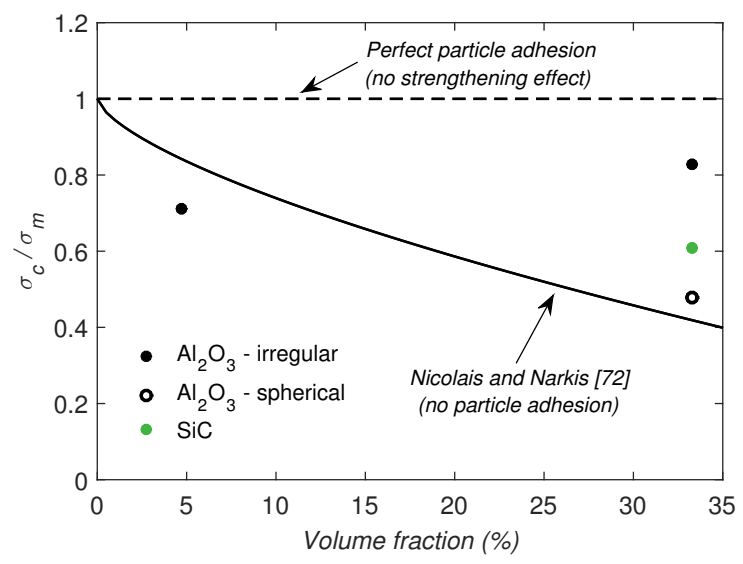

Figure 85. Relative tensile strength of various particulate composites along with particle adhesion models.

The adhesion strength between filler and matrix becomes more important as the particle $A R$ decreases [54]. Tensile stresses are more efficiently transferred to fillers having large surface areas, resulting in higher tensile strength. The shape and $A R$ of irregular particles provide a larger surface area-to-volume ratio $(A / V)$ relative to spheres, which encourages efficient particle-matrix adhesion; however, small variations in $A R$ such as those found in the present study $(A R \approx 1-10)$ are not expected to increase $A / V$ significantly. The relation between $A / V$ and $A R$ can be better visualized through Fig. 10. Therefore, the large stress concentration factors provided by the edges of our irregular particles are expected to have a much more dominant effect on the dynamic response of $\mathrm{Al}_{2} \mathrm{O}_{3}$-PDMS composites as compared to the slightly improved particle adhesion efficiency resulting from higher $A / V$.

As noted by the quasi-static results in Fig. 83 and 85, the spherical $\mathrm{Al}_{2} \mathrm{O}_{3}$-PDMS demonstrated significantly lower strength compared to its irregular counterpart. Since identical 
surface chemistry should be expected as well as relatively similar $A R$ values, the large differences in composite strength may be a result of improper particle wetting. Spherical particles were suspected to suffer from poor wetting due to nanoparticle agglomeration (see Section 5.4), although the extent of this effect is unknown. This may have further decreased its ballistic resistance along with the particle geometry effect. Additional experimentation is required to properly validate the current results.

\subsection{Projectile Nose Geometry Study}

The ballistic response of composites impacted by RCC projectiles has been thoroughly assessed in previous sections. These results shall be used as a baseline to compare the behaviour of composites penetrated by chisel-nosed projectiles, which have a more narrow flat-nosed region (see Fig. 44). The results of composites penetrated by hemispherical projectiles will be discussed subsequently. The use of conical-nosed penetrators has been limited due to excessive projectile instabilities and will not be discussed. Residual velocity data for PDMS and 33.3 vol.\% SiC-PDMS can however be found in Appendix A. Signs of yaw were noted, although instabilities in the orthogonal plane could not be identified. In order to properly assess the effect of projectile nose geometry, all projectiles had a constant mass (16-grains/projectile). The shank diameter was also held constant while slightly varying its length to satisfy the mass requirement.

\subsubsection{Chisel-nosed Projectiles}

The effect of particle content for composites impacted by chisel-nosed projectiles has been investigated in order to note differences in response relative to RCC projectiles. Based on geometry, the area ratio $\left(A_{r}\right)$ between the flat-nosed regions of these two projectiles is 0.58 [44]. The residual velocity data and the corresponding $\kappa$ values are shown in Fig. 86. As

opposed to the results from RCC projectiles, Fig. 86b shows that all systems outperformed their inertial response. The neat PDMS and the 4.7 vol.\% SiC-PDMS composite converged 


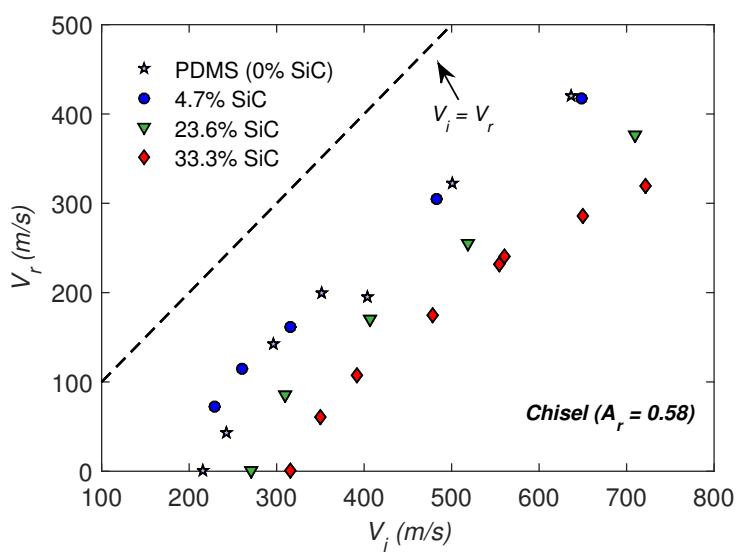

(a)

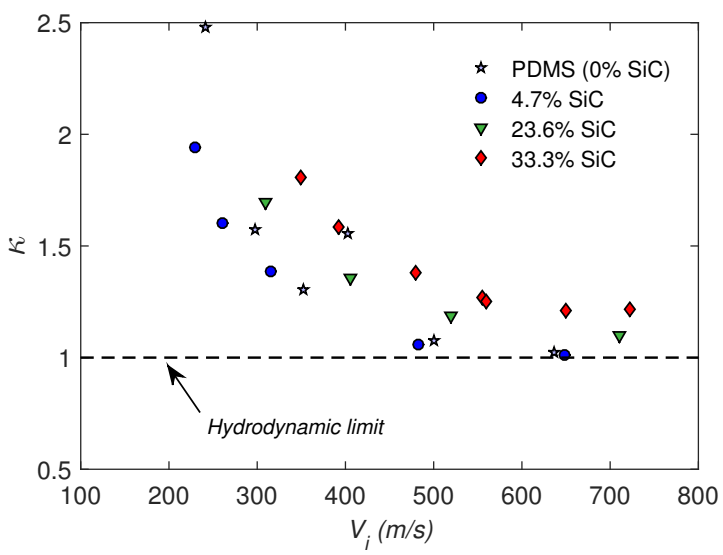

(b)

Figure 86. Volume fraction effect for SiC-PDMS impacted by chisel-nosed projectiles: (a) $V_{r}-V_{i}$ curves and (b) normalized performance parameter.

to the hydrodynamic limit at high impact velocities while composites having higher particle concentrations showed signs of dynamic material strength $(\kappa>1)$ over the entire testing range. Ballistic limit velocities were extracted from Fig. 86a. The penetration response of the composites was compared to the RI plugging model using $A_{r}=0.58$, as seen in Fig. 87a. The plugging fraction parameter was included in Fig. 87b to further emphasize the correlation of the data with respect to the plugging model. Excellent agreement was seen for the 23.6 vol.\% composite, however at higher particle loading, the normalized velocity data exceeded the upper limit (i.e., $\left.p_{f}>1\right)$. This suggests that the area ratio $\left(A_{r}=0.58\right)$ may not be representative of the plugging event or that the RI plugging model does not account for a certain behaviour that further enhances the ballistic resistance of the composites. It should be noted that the angled surfaces of the chisel-nosed projectiles (Fig. 44) were not considered in the RI plugging model and could therefore be the source of this discrepancy.

An interesting response was observed when comparing the residual velocity curves for RCC and chisel-nosed projectiles penetrating the 4.7 vol.\% SiC-PDMS near $V_{b l}$. These data sets are emphasized in Fig. 88 for clarity along with the neat PDMS data. It can be noted that the $V_{b l}$ of chisel-nosed projectiles decreased when a low concentration of particles was added whereas the opposite was found for RCC projectiles. The trend is illustrated in Fig. 


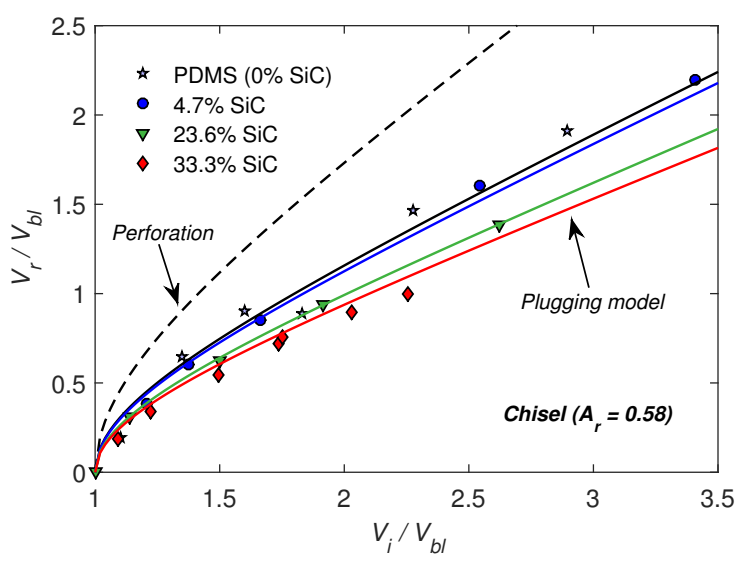

(a)

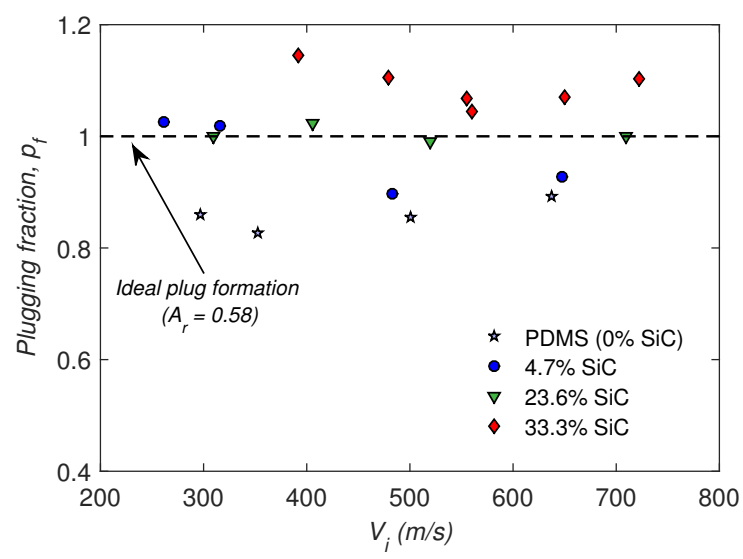

(b)

Figure 87. Volume fraction effect for SiC-PDMS impacted by chisel-nosed projectiles: (a) $\mathrm{V}_{b l}$-normalized residual velocity data with $\mathrm{RI}$ plugging models and (b) plugging fraction parameter.

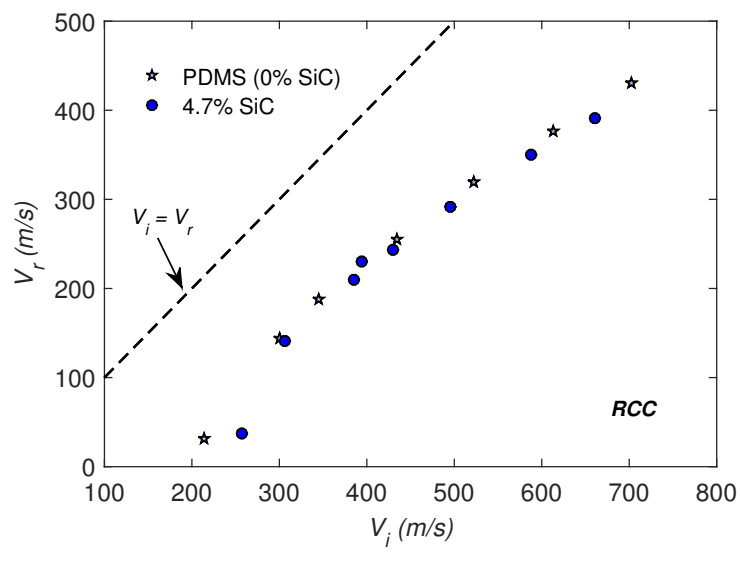

(a)

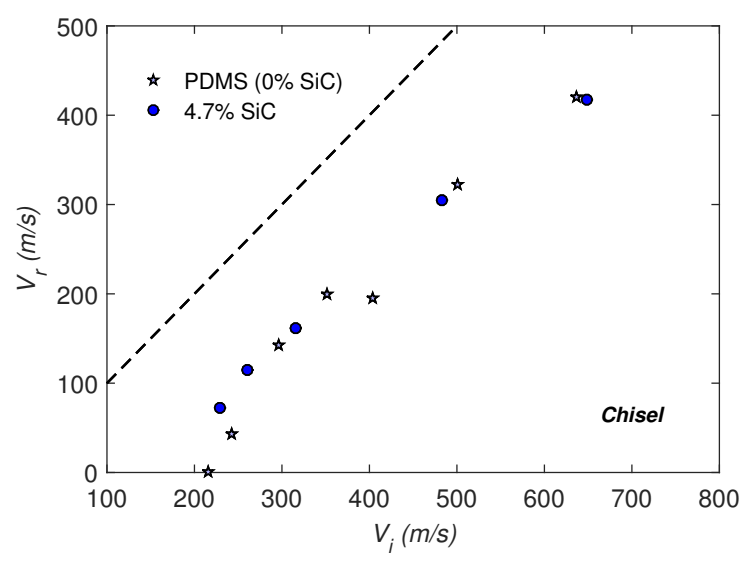

(b)

Figure 88. Projectile nose geometry effect on 4.7 vol.\% SiC-PDMS: $V_{r}-V_{i}$ cuves.

89, which plots the estimated $V_{b l}$ for SiC-PDMS composites at varying particle content. Nevertheless, extensive variability in residual velocity data can occur near the ballistic limit. Additional experimentation at this velocity range is required to confirm the trend observed for chisel-nosed projectiles since the dataset is limited in this case. The increased $V_{b l}$ trend resulting from RCC penetration is however supported by experiments on different composites (see Fig. 68a and 69a).

The residual velocity data for RCC and chisel-nosed projectiles impacting 33.3 vol.\% composites having different filler materials is shown in Fig. 90a. The ballistic limit velocity 


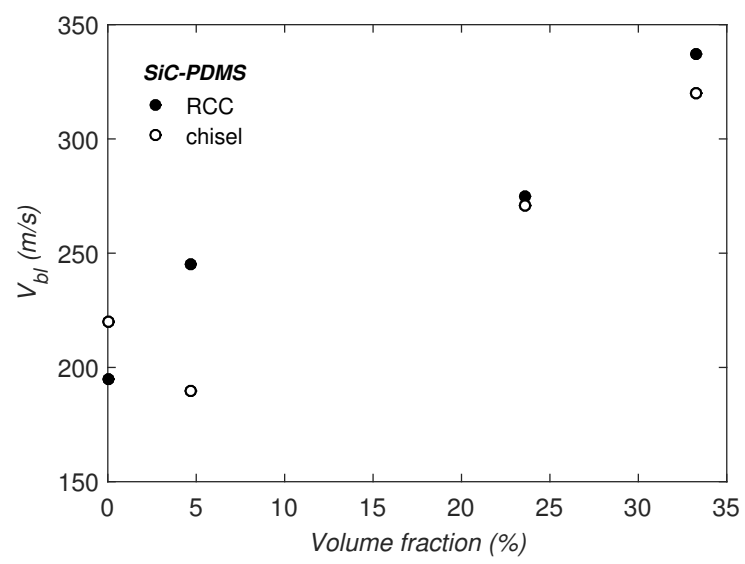

Figure 89. Estimated ballistic limit velocity of SiC-PDMS composites having varying particle volume fraction impacted by RCC and chisel-nosed projectiles.

was relatively insensitive to the variations in projectile nose (within experimental error) while a large difference was observed based on the choice of filler materials. The estimated $V_{b l}$ values for each composite-projectile combination are found in Table 7 . The influence of

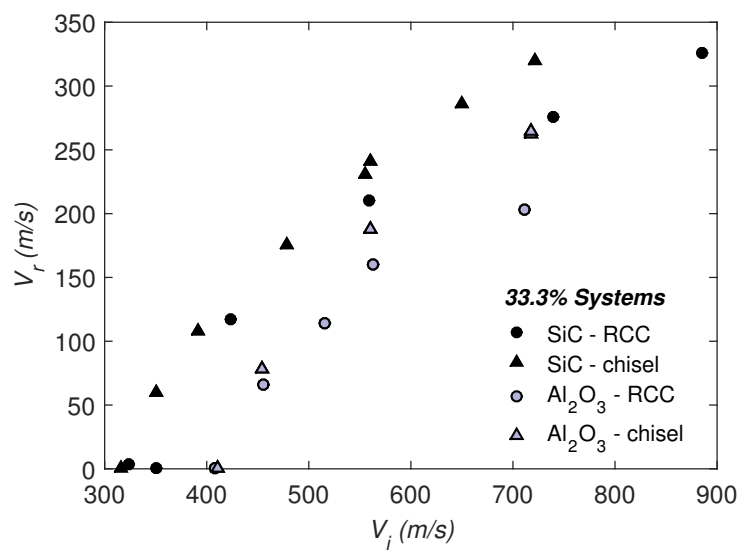

(a)

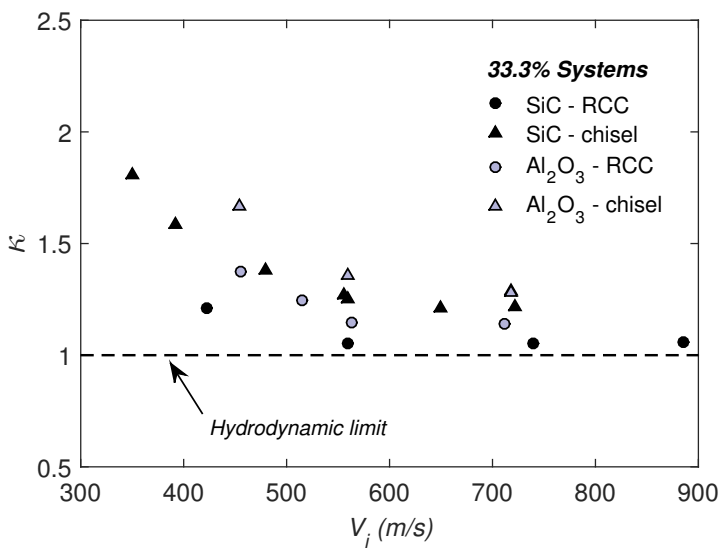

(b)

Figure 90. Effect of projectile nose shape for two 33.3 vol.\% composites: (a) $V_{r}-V_{i}$ curves and (b) normalized performance parameter.

projectile nose was more evident at high impact velocities. The difference in residual velocity data for RCC and chisel-nosed projectiles was likely the result of inertial contributions. The larger nose area of RCC projectiles has the potential to transfer more axial momentum to the target material (ejecta), therefore further lowering $V_{r}$. Effective momentum transfer occurred at high impact velocities. The dynamic response of composites was further assessed through 
the normalized performance parameter $\kappa$, which is plotted in Fig. 90b. RCC projectiles experienced more deceleration for a given impact velocity, although chisel-nosed projectiles displayed better performance relative to their inertial response, as evidenced by their higher $\kappa$ values. It should be noted that the superior performance of targets penetrated by chiselnosed projectiles may once again be linked to the choice of area ratio, $A_{r}=0.58$.

Table 7. Estimated ballistic limit velocities, $V_{b l}(\mathrm{~m} / \mathrm{s})$, for 33.3 vol.\% particulate composites impacted by RCC, chisel-nosed, and hemispherical projectiles.

\begin{tabular}{lccc}
\hline Composite (33.3 vol.\%) & RCC & Chisel & Hemispherical \\
\hline SiC-PDMS & 337 & 320 & 275 \\
$\mathrm{Al}_{2} \mathrm{O}_{3}$-PDMS & 415 & 411 & 340 \\
\hline
\end{tabular}

The ballistic response of 33.3 vol.\% composites penetrated by both projectiles was correlated to the RI plugging models, as seen in Fig. 91. Contrary to the results from RCCs, the entire dataset for chisel-nosed projectiles was below the theoretical range of acceptable $V_{r} / V_{b l}$ values. Figure $92 \mathrm{a}$ and $92 \mathrm{~b}$ compare the $V_{r} / V_{b l}$ data of both composites for a given

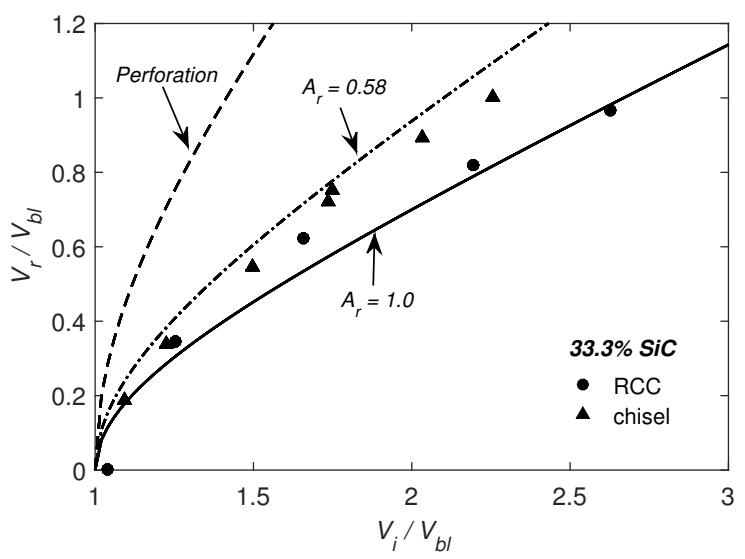

(a)

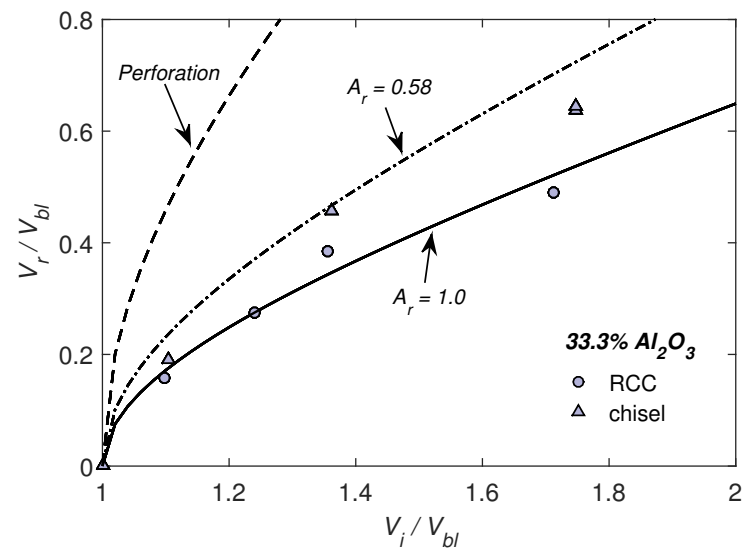

(b)

Figure 91. $\quad V_{b l}$-normalized residual velocity curves for 33.3 vol.\% particulate composites impacted by RCC and chisel-nosed projectiles. Data correlated to RI plugging models: (a) $\mathrm{SiC}-\mathrm{PDMS}$ and (b) $\mathrm{Al}_{2} \mathrm{O}_{3}$-PDMS.

projectile (chisel-nosed and RCC, respectively). The normalized velocity data for both materials impacted by chisel-nosed projectiles fell onto a single curve below $V_{i} / V_{b l} \approx 1.5$, but diverged at higher values. The two right-most (overlapping) data points for $\mathrm{Al}_{2} \mathrm{O}_{3}-\mathrm{PDMS}$ 
in Fig. 92a demonstrate that this deviation from the remaining $V_{r} / V_{b l}$ data was consistent and was not a consequence of scatter. A similar decrease in $V_{r} / V_{b l}$ was observed for both materials impacted by RCC projectiles in Fig. 92b. This phenomenon was only observed for composites impacted by flat-nosed penetrators (above a certain $V_{i}$ value). It is suggested that a change in target-projectile interaction occurred or that blunt projectiles induced a transition in material response. An increased stress state or strain-rate sensitivity may be the cause for such phenomenon. Consequently, the fact that the RI plugging model assumes constant work during penetration seems to be incorrect for the particulate composites under investigation. More experimentation is required to properly validate the trend.

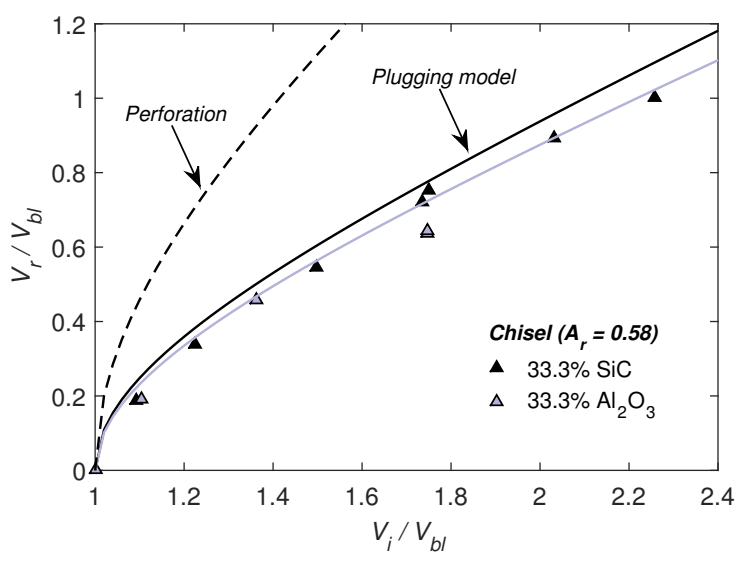

(a)

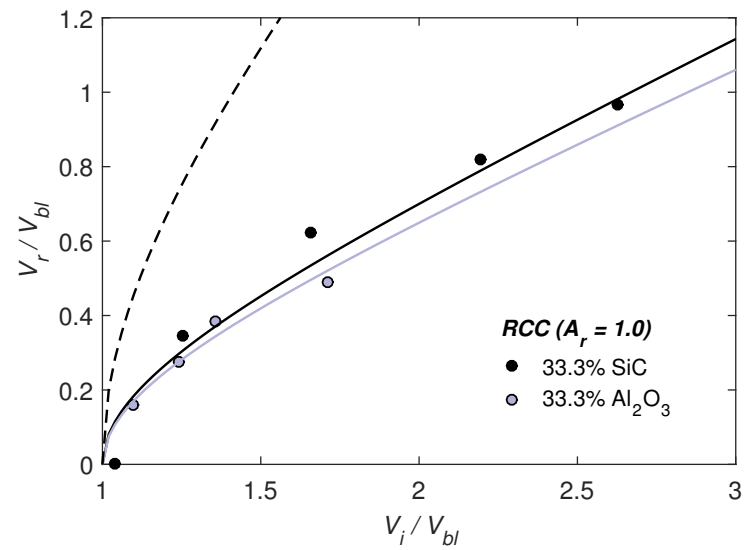

(b)

Figure 92. $V_{b l}$-normalized residual velocity curves and corresponding RI plugging models for 33.3 vol.\% SiC- and $\mathrm{Al}_{2} \mathrm{O}_{3}$-PDMS impacted by: (a) chisel-nosed and (b) RCC projectiles.

The constant work assumption can be verified by calculating the average resistive shear stresses for the different target-projectile combinations, as shown in Fig. 93. Lateral expansion of the target cavity was assumed to be restricted for the 33.3 vol.\% systems, enabling the use of Eq. 34. Targets penetrated by chisel-nosed projectiles appeared to exhibit a constant $\tau_{a v}$ value below $V_{i} \approx 600 \mathrm{~m} / \mathrm{s}$, followed by a significant increase at higher impact velocities. On the other hand, those penetrated by RCC projectiles experienced an initial decrease in $\tau_{a v}$. A minimum was reached near $V_{i} \approx 550 \mathrm{~m} / \mathrm{s}$, followed by an increase afterwards, which was similar to the response provided by chisel-nosed projectiles. The present 
analysis demonstrated a velocity dependent resisting stress, therefore a constant penetration work is not expected to be an accurate assumption.

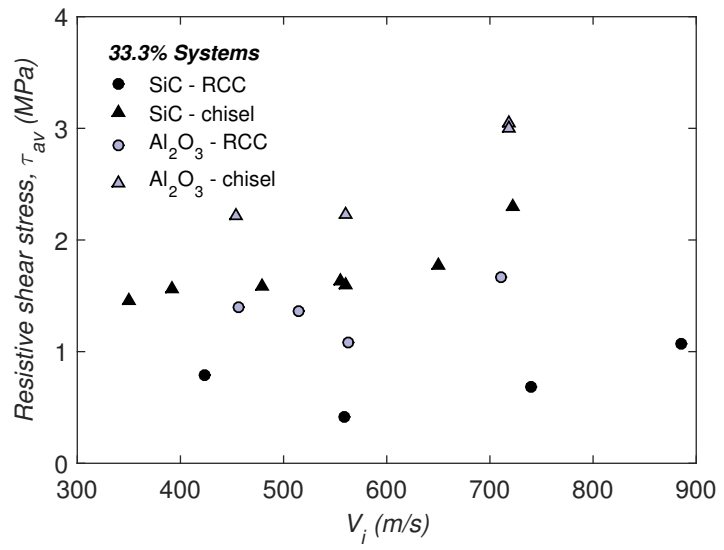

Figure 93. Projectile nose geometry effect for 33.3 vol. $\%$ SiC- and $\mathrm{Al}_{2} \mathrm{O}_{3}-\mathrm{PDMS}$ systems: average resistive shear stress.

\subsubsection{Hemispherical Projectiles}

Hemispherical projectiles are considered to represent an intermediate case between the penetration of sharp and flat-nosed projectiles [81]. The residual velocity curves for 33.3 vol.\% SiC- and $\mathrm{Al}_{2} \mathrm{O}_{3}$-PDMS composites impacted by hemispherical projectiles is shown in Fig. 94a along with the response of neat PDMS. A significant difference in $V_{b l}$ was noticed, whereas the variations in residual velocity were minimized at higher impact velocities. $\mathrm{Al}_{2} \mathrm{O}_{3}-\mathrm{PDMS}$ composites exhibited enhanced stiffness and strength over their SiC-filled counterpart when tested under quasi-static conditions (Fig. 53 and 55). The large discrepancy in material response was attributed to superior interfacial adhesion between the matrix and $\mathrm{Al}_{2} \mathrm{O}_{3}$ particles. Additional work may have been required for void nucleation and subsequent tensile failure ahead of the hemispherical nose. The material behaviour was compared to the RI perforation model in Fig. 94b. As opposed to the results from flat-nosed projectiles, the normalized velocity data of both composites could be described by a single curve over the entire range of impact velocities. The response of neat PDMS did not follow this curve, although it was not properly represented by the perforation model either. 


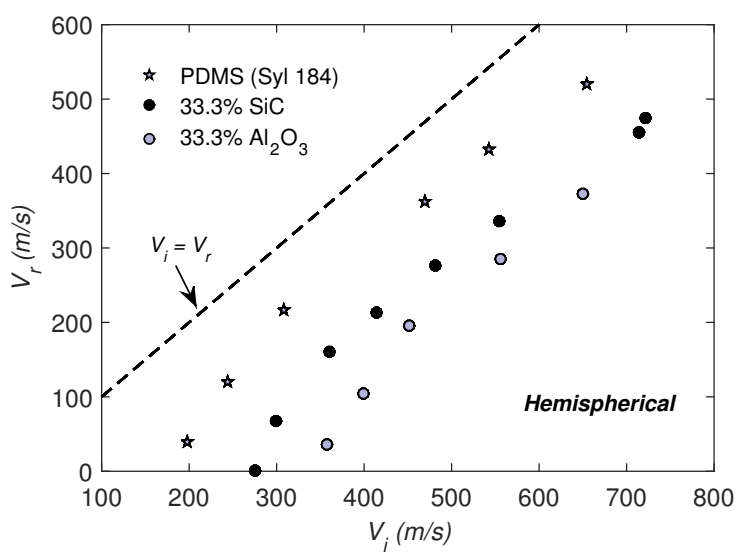

(a)

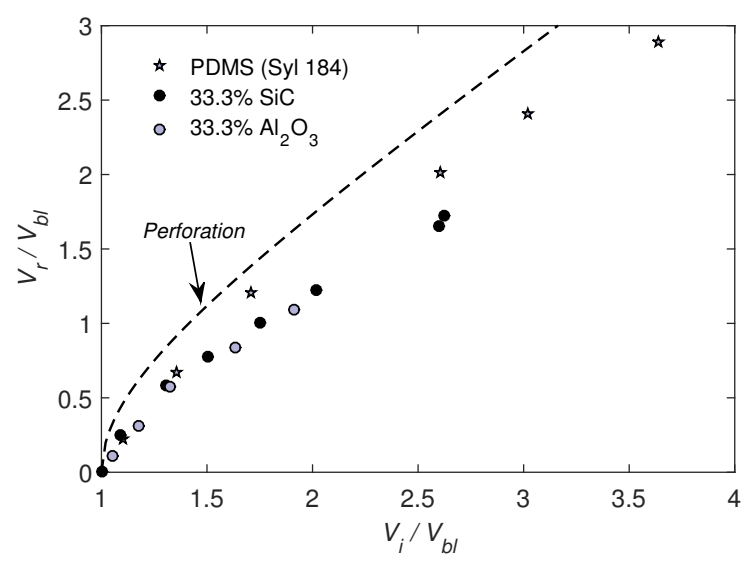

(b)

Figure 94. Ballistic results showing effect of particle integration against penetration of hemispherical projectiles.

The ballistic response of 33.3 vol.\% particulate composites impacted by hemispherical and RCC projectiles is compared in Fig. 95. The $V_{b l}$ of a material impacted by hemispherical projectiles was considerably lower than that of RCCs, as seen in Table 7. The correlation

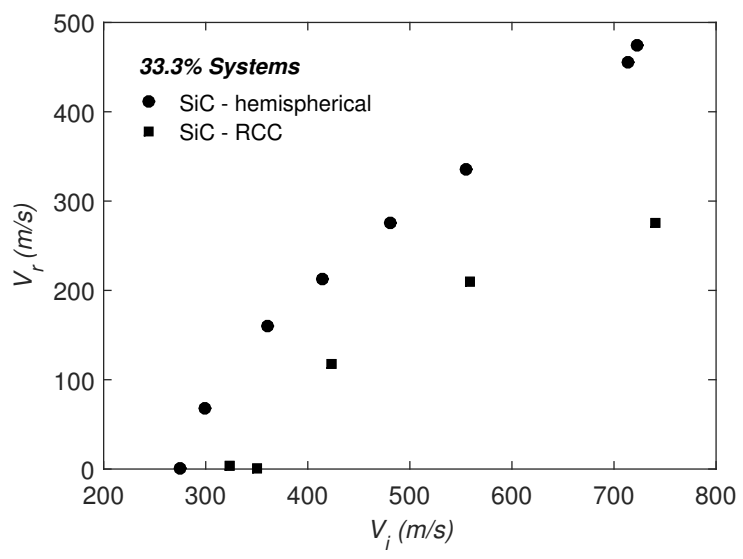

(a)

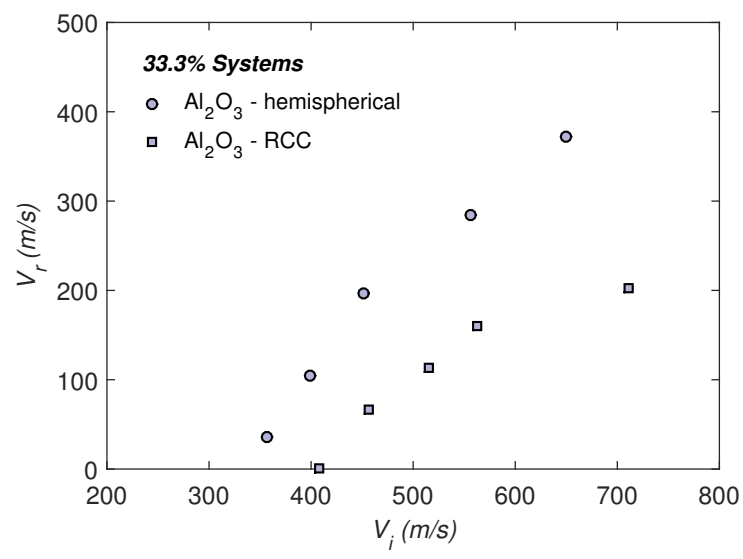

(b)

Figure 95. Residual velocity curves for 33.3 vol.\% particulate composites impacted by hemispherical and RCC projectiles: (a) SiC-PDMS and (b) $\mathrm{Al}_{2} \mathrm{O}_{3}$-PDMS.

to the plugging models for both of these projectiles are shown in Fig. 96a. The differences between both plugging models comes from assuming that no energy is absorbed by plastic deformation during the impact of hemispherical projectiles [81]. This assumption provided an adequate representation of the material response and could be described by a constant $p_{f}$ 
value away from $V_{b l}$, as seen in Fig. 96b. The addition of particles increased the correlation to the plugging model compared to neat PDMS. This suggests that lateral expansion of the material was partially restricted by the stiffer response, allowing for additional axial momentum transfer. The constant $p_{f}$ values for hemispherical projectiles at high impact velocities suggest that different penetration mechanisms were present, as compared to flatnosed projectiles.

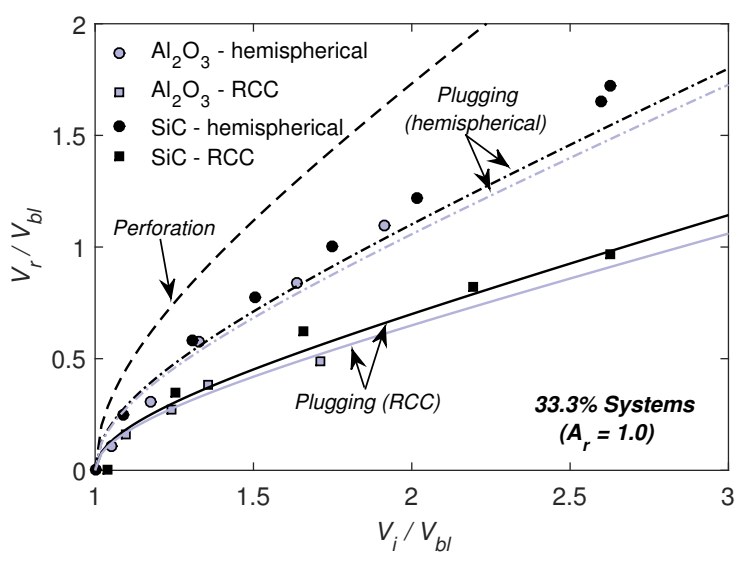

(a)

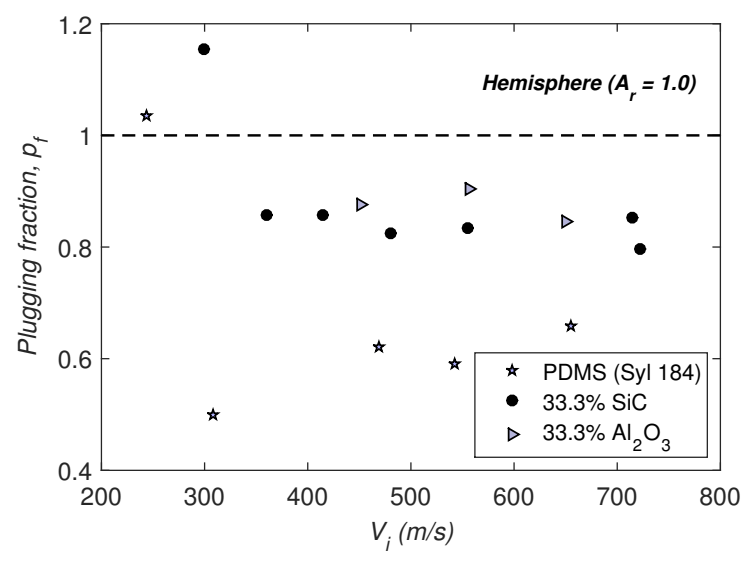

(b)

Figure 96. Correlation with plugging models: (a) $V_{b l}$-normalized residual velocity curves for $33.3 \mathrm{vol} . \%$ particulate composites impacted by hemispherical and RCC projectiles and (b) plugging fraction parameter for composites impacted by hemispherical projectiles.

As an extension of the particle morphology study, spherical 33.3 vol. $\% \mathrm{Al}_{2} \mathrm{O}_{3}$-PDMS composites were also penetrated by hemispherical projectiles. The trends in results relative to impact by RCC projectiles were very similar to those observed in the present section and will not be further discussed. The data can be found in Appendix A.

\subsection{Ballistic Testing: Conclusions}

The ballistic response of particle-filled elastomers was characterized to determine the role played by various particulate parameters. Residual velocity data were analyzed through several theoretical models and the conclusions are summarized below. The reader is referred to Appendix A and B for the complete ballistic dataset. 


\subsubsection{Ballistic Response of PDMS}

Ballistic limit velocities were estimated for neat PDMS penetrated by three projectile nose geometries. The results in Table 6 showed that chisel-nosed projectiles exhibited the highest $V_{b l}$ while hemispherical geometries produced the lowest value. The differences in $V_{b l}$ values were however relatively small, i.e., within $30 \mathrm{~m} / \mathrm{s}$ from each other.

Figure 61d showed that RCC projectiles experienced the highest deceleration at elevated impact velocities. Neat PDMS suffered from significant lateral expansion when penetrated by RCCs, resulting in limited axial momentum transfer to the target material. This was confirmed by the lack of agreement between its ballistic data and the RI plugging model, Fig. 64. Further evidence was provided by the ejecta fields in Fig. 65. A schematic of the suggested penetration event for RCC projectiles was illustrated in Fig. 62 and shows that a cavity may be forming ahead of the flat nose due to the elasticity of PDMS and lateral expansion effects. On the other hand, targets appeared to suffer less from lateral expansion when penetrated by chisel-nosed projectiles since their response was adequately represented by the RI plugging model. Lateral expansion effects may have caused the PDMS to underperform relative to its hydrodynamic response when impacted by RCCs, as seen in Fig. 66. Improper interfacial contact between projectile nose and target was also suggested to have negatively influenced its dynamic performance.

\subsubsection{Effect of Particle Volume Fraction}

A moderate increase in the $V_{b l}$ of PDMS impacted by RCCs was noted with the integration of particles at 4.7 vol.\%. The low particle content offered negligible improvements in ballistic resistance at elevated impact velocities while composites having higher volume fractions experienced superior performance over the entire range of velocities. The latter response may have resulted from particles restricting lateral motion during penetration and increasing axial acceleration of the material, as evidenced by the ejecta fields observed in Fig. 72. Consequently, composites approached a plugging response with increasing particle content 
(see Fig. 74 and 75). The 33.3 vol.\% composites were able to recover their inertial response at high impact velocities. High particle content may have also improved projectile-target contact. A schematic illustrating the idealized penetration event of composites having high particle concentration is shown in Fig. 71.

\subsubsection{Effect of Particle Material Type}

The filler material type was found to have a considerable influence on the ballistic response of 33.3 vol.\% composites. Ballistic resistance seemed to increase with increasing particle density (Fig. 76). This trend remained unaltered after normalizing the dynamic response with respect to its inertia, suggesting that the influence of composite density may not have been properly accounted for. Increased density could have provided additional penetration work through cavitation considerations, or by improving interfacial contact between projectile and target. Results suggest that the ballistic resistance of composites was not strongly influenced by other filler material properties at the volume fractions considered, therefore interparticle contact was not a dominant reinforcing mechanism. Figure 77 shows a substantial increase in $V_{b l}$ for $\mathrm{Al}_{2} \mathrm{O}_{3}$-PDMS relative to other composites, suggesting that superior interfacial adhesion between particles and matrix may have played a significant role.

Material filler effects were further investigated by comparing the results of 33.3 vol.\% composites having different capsule lengths in order to maintain a constant areal density. Equivalent axial momentum transfer can be assumed, therefore the differences in material response were due to varying contributions from penetration work. As seen in Fig. 80, the average resisting shear stress of $\mathrm{Al}_{2} \mathrm{O}_{3}$-PDMS was superior compared to other composites. Although the resistive stresses of all materials increased with impact velocity, the $\mathrm{Al}_{2} \mathrm{O}_{3^{-}}$ PDMS showed a higher degree of sensitivity. Superior ballistic resistance was attributed to improved particle adhesion or better target-projectile contact. The longer $\mathrm{B}_{4} \mathrm{C}-\mathrm{PDMS}$ however exhibited the highest $V_{b l}$, demonstrating that capsule length was a more significant factor at low impact velocities. Overall, the ballistic response of composites having a constant 
$A_{d}$ were quite similar, which elucidates the dominating contribution of target inertia.

\subsubsection{Effect of Particle Morphology}

A strong influence from particle morphology was observed for $33.3 \mathrm{vol} . \% \mathrm{Al}_{2} \mathrm{O}_{3}$-PDMS in Fig. 81. An irregular morphology provided significantly superior ballistic resistance compared to spherical particles. This was primarily attributed to the shape and $A R$ of particles and their interactions with the matrix. Although the irregular particles are likely to offer a slightly higher $A / V$ ratio, the small increase in interfacial adhesion efficiency is expected to be negligible in comparison to the shape effect. Both composites were adequately described by the RI plugging model, although a consistent rise in resistive shear stress was noted at $V_{i} \approx 700 \mathrm{~m} / \mathrm{s}$, Fig. 82. The latter may indicate a transition in the dynamic response of composites or that the contact with the projectile nose has been improved.

The response of spherical $\mathrm{Al}_{2} \mathrm{O}_{3}$-PDMS was compared to SiC-PDMS to verify whether its irregular particle morphology provided similar improvements (Fig. 84). This was not the case, since the $V_{r}-V_{i}$ data for both composites penetrated by RCC projectiles were very similar, resulting in seemingly identical $\tau_{a v}$ values over the entire velocity range. A slight increase in $\tau_{a v}$ was however observed for SiC-PDMS over spherical $\mathrm{Al}_{2} \mathrm{O}_{3}-\mathrm{PDMS}$ when impacted by chisel-nosed projectiles, which may be a sign of particle geometry effects. On the other hand, the irregular $\mathrm{Al}_{2} \mathrm{O}_{3}$-PDMS demonstrated significantly higher resistive stress when penetrated by both projectiles. It was assumed that although morphology had a strong influence on ballistic performance, a competing effect arises from particle adhesion considerations. Therefore, strong interfacial adhesion strength seems to be required in order to maximize the contribution provided by irregular-shaped particles.

\subsubsection{Effect of Projectile Nose Geometry}

The response of composites impacted by RCCs was compared to that obtained from chiselnosed projectiles. Contrary to RCCs, the response of neat PDMS and 4.7 vol.\% SiC-PDMS 
converged to the hydrodynamic limit at high impact velocities while composites loaded to higher volume fractions showed superior dynamic strength over the entire velocity range, Fig. 86. The projectile nose shape seemed to have a strong influence on the $V_{b l}$ of 4.7 vol.\% SiC-PDMS. Interestingly, the $V_{b l}$ of chisel-nosed projectiles was found to decrease relative to neat PDMS while the opposite was observed for RCCs, Fig. 88. This nose shape effect was minimized at higher volume fractions, Fig. 89, although the $V_{b l}$ values resulting from RCCs were consistently higher.

Differences in residual velocity data for 33.3 vol.\% composites were attributed to the dominating inertial contribution resulting from different flat-nosed areas. The $V_{r} / V_{b l}$ data resulting from chisel-nosed projectiles fell below the theoretical range of values expected from a shear plugging response $\left(p_{f}>1\right)$, suggesting that the penetration event was not properly described by the chosen area ratio, $A_{r}=0.58$. Consequently, their $\kappa$ values were consistently higher than those of RCCs, as seen in Fig. 90b. This may have resulted from neglecting contributions from the angled surfaces of chisel-nosed projectiles.

The constant work assumption adopted by the RI plugging model was investigated for both flat-nosed projectiles by calculating the average resistive shear stress $\tau_{a v}$ exerted by 33.3 vol.\% composites. According to this analysis, the resistive shear stress was velocitydependent and demonstrated signs of dynamic strengthening at high $V_{i}$ values. Therefore, constant penetration work is not an accurate assumption for the 33.3 vol.\% particulate composites, although the dominating influence of inertia permits the RI plugging model to provide a decent rough estimate of the response.

Residual velocity data were obtained for 33.3 vol.\% composites penetrated by hemispherical projectiles and compared to the results from RCCs. Table 7 demonstrates that a rounded nose resulted in significantly lower $V_{b l}$ values compared to flat-nosed projectiles. This reveals a significant weakness for these composites. Filler material type significantly affected $V_{b l}$, which may be a result of differences in particle adhesion. Regardless, the $V_{r} / V_{b l}$ data obtained from different particulate composites collapsed onto a single curve over the entire 
velocity range. The same could not be said for flat-nosed projectiles, since deviations were observed at high velocities. 


\section{Post-mortem Analysis of Ballistic Capsules}

In the previous chapter, we analyzed the ballistic response of particulate composites using residual velocity data extracted from capsule penetration experiments. Theoretical models based on conservation of energy and momentum principles were employed as an attempt to describe the penetration event, the failure mechanisms that took place, and the role of the filler material. Several particle parameters were found to affect the ballistic resistance of particulate composites. It is difficult to isolate and experimentally study the effect of a single material property without altering another aspect of the composite. For example, increasing the filler volume fraction also led to an increased composite density; different particle material types varied the strength and stiffness of filler inclusions, but also their density and adhesive properties; and particle morphology may have affected its wetting characteristics. This led to many observations concerning the role played by the particulates within an elastomeric matrix. The present chapter revisits the ballistic response of these elastomeric systems through a qualitative (visual) approach. The objective is to confirm some of the claims that were previously suggested and to further describe the failure mechanisms observed through post-mortem images of impact surfaces and penetration channels.

\subsection{Ballistic Response of PDMS Elastomer}

The residual velocity data of neat PDMS penetrated by different projectiles was analyzed in Section 6.1. Excluding the results from conical projectiles (due to a lack of reliable data points), the range of $V_{b l}$ for different projectiles was estimated to be within approximately $40 \mathrm{~m} / \mathrm{s}$ of each other (see Table 6). Due to the transparency of neat PDMS, the penetration channels created by different projectiles could be observed without dissecting the capsules. These shall first be examined for impact velocities near $V_{b l}$, followed by higher velocity impacts.

Figure 97 shows two PDMS capsules penetrated by chisel-nosed projectiles at velocities 
(a) slightly below and (b) slightly above $V_{b l}$. The PDMS appeared to have failed in shear, resulting in a slightly oval-shaped penetration channel, which is consistent with the flatnosed geometry of the projectile (Fig. 44). As evident from Fig. 97a, even the larger dimension of the oval-shaped channel was smaller than the diameter of the projectile. This observation confirms that the PDMS tends to expand laterally away from the projectile nose as it is penetrated, therefore shearing less material. A cavity resulting from shear plugging was found at the back surface of the target (Fig. 97b) when the projectile impacted PDMS slightly above $V_{i}=V_{b l}$. It is interesting to note that the cavity was approximately the same diameter as the projectile, signifying that lateral expansion was less dominant near the back surface.

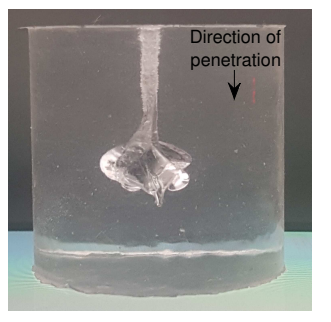

(a)

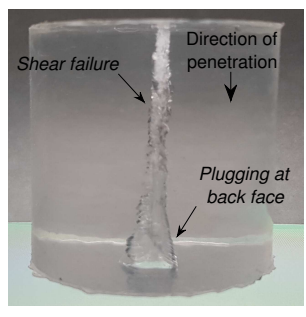

(b)

Figure 97. Image of neat PDMS impacted by chisel-nosed projectile at impact velocities (a) slightly below and (b) slightly above $V_{b l}$.

The penetration channel resulting from penetration by RCC projectiles was slightly different. As RCCs decelerated throughout the PDMS targets near $V_{b l}$, the failure mechanism was seen to transition from shear to tensile failure, Fig 98. The projectile appears to have initially sheared through the target until a threshold velocity was reached. At this point, the RCC was no longer able to fully shear the material around its entire circumference, resulting in the initiation of a single semi-circular crack at a portion of the projectile edge. The RCC expanded the crack through tensile fracture and slipped through the propagating plane for the remainder of the penetration event. This is expected to have relieved the resisting pressure acting on the projectile nose. The transition was confirmed to be a velocity-dependent phenomenon since lower velocity impacts resulted in an earlier transition in failure mecha- 
nism, as seen in Fig. 98a and 98b. Evidence of the semi-circular tensile fracture channel is provided by a magnified isometric view of the target, Fig. 98c. This may explain why RCCs had a lower $V_{b l}$ value compared to chisel-nosed projectiles, since the latter experienced shear failure throughout the entire penetration. In terms of work invested during penetration,

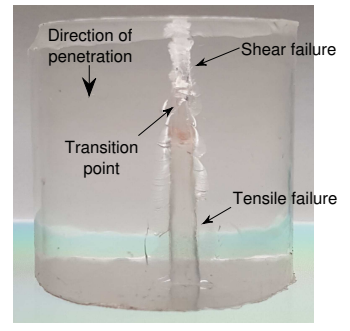

(a)

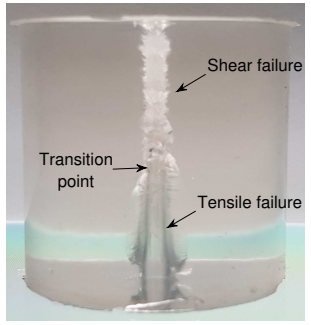

(b)

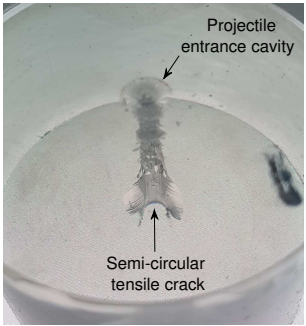

(c)

Figure 98. Images of neat PDMS penetrated by RCC projectiles, demonstrating transition in failure mechanism throughout penetration channel (shear to tensile failure): (a) $V_{i}=214$ $\mathrm{m} / \mathrm{s}$, (b) $V_{i}=300 \mathrm{~m} / \mathrm{s}$, and (c) isometric view, $V_{i}=300 \mathrm{~m} / \mathrm{s}$.

it may no longer have been favourable for the larger flat portion of the RCC to continue shearing the material below some threshold velocity, therefore a transition to tensile failure occurred. This may have resulted from diminished axial compression ahead of the projectile nose, which altered the complex stress state of the material. Such an event is somewhat contradictory to what would be expected from thick metallic plates. Based on the analysis conducted by Woodward [107, 106], ductile hole enlargement is preferred for the initial penetration stage, followed by shearing near the back surface due to diminishing shear area. The event described by Woodward was however a consequence of geometry between target and projectile, while the transition in failure mechanisms that was suggested for particulate composites is dependent on both geometry and velocity. This may be due to the fact that metals have much higher fracture toughness compared to polymers, thus, penetration through crack propagation is not a plausible option.

Shear failure was not observed when PDMS was penetrated by hemispherical projectiles. Large tensile forces were generated along its rounded nose, followed by crack initiation at the tip in order to relieve stress. Bending stresses induced by the projectile then further 
expanded the crack (axially) to establish its penetration path and slip through the cavity. This tensile failure mode is likely the cause for the lower $V_{b l}$ value compared to chisel-nosed projectiles (Table 6), although the differences are not drastic. The single tensile crack failure mechanism is demonstrated in Fig. 99. It should be noted that the material experienced radial cracking due to excessive lateral expansion in the initial penetration stages and should not be mistaken for shear failure.

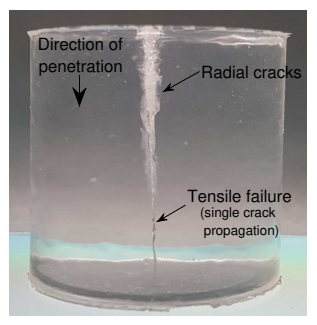

Figure 99. Image of neat PDMS impacted by hemispherical projectiles near $V_{b l}$, showing a single tensile crack failure mechanism.

The penetration channels of PDMS were significantly altered at high impact velocities. Large radial (tensile) cracks spanning the length of the capsules were found around the circumference of penetration channels. The size of these cracks were dependent on projectile nose shape, but especially on impact velocity. The cracks provide evidence of excessive lateral expansion (cavitation) during penetration, followed by rapid decompression of the material. The dependence of impact velocity on crack size is demonstrated for RCC projectiles in Fig. 100. Cavitation is a phenomenon first observed in metals that causes a larger penetration channel diameter than that of the projectile, e.g., Fig. 17 [87]. For the systems under investigation, cavitation is manifested through these radial cracks and not large permanent deformation since elastomers exhibit high recoverability. It is assumed that the cracks were initiated during penetration of the projectile and grew extensively afterwards due tensile hoop stresses generated from cavitation effects. This is evidenced by Fig. 101 which shows cracks propagating radially outwards from the sharp corners of a chisel-nosed projectile (stress concentrations). The threshold velocity for cavitation $V_{\text {cav }}$ is dependent on the resistive 


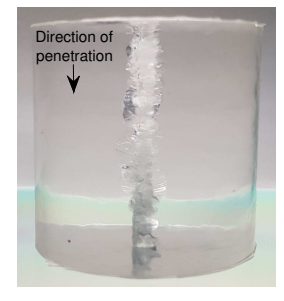

(a)

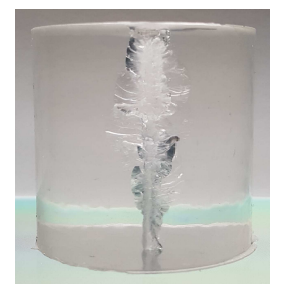

(b)

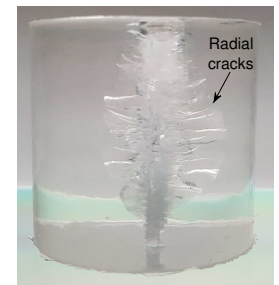

(c)

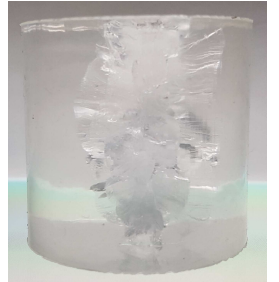

(d)

Figure 100. Images of neat PDMS capsules impacted by RCC projectiles at: (a) $V_{i}=345$ $\mathrm{m} / \mathrm{s}$, (b) $V_{i}=435 \mathrm{~m} / \mathrm{s}$, (c) $V_{i}=613 \mathrm{~m} / \mathrm{s}$, and (d) $V_{i}=888 \mathrm{~m} / \mathrm{s}$. Results show the progression of radial cracking with increasing velocity.

stress of the target, its density, and projectile nose shape (Eq. 14). Since PDMS is very compliant to deformation, its resistive stress must be very low, therefore, so should $V_{\text {cav }}$. The crack patterns and radial lengths found in different targets may provide valuable information concerning the ballistic performance of a composite. For example, the RCC projectiles resulted in the largest radial cracks, signifying the most intense lateral expansion. This is consistent with the results from Rosenberg and Dekel [86], who found that RCC projectiles were the most prone to cavitation.

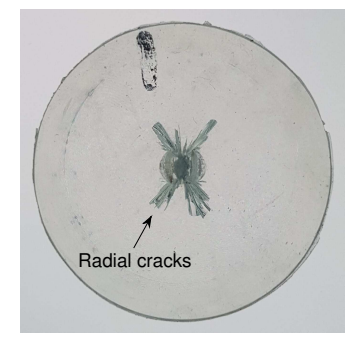

(a)

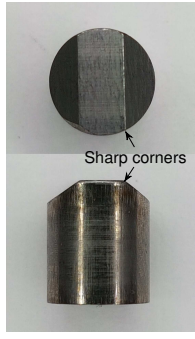

(b)

Figure 101. (a) Post-penetration image of neat PDMS impact surface. Radial cracks propagate from each corner of a chisel-nosed projectile. The projectile corners act as stress concentrations, which initiate cracks during penetration. The cracks then grow due to excessive lateral expansion (cavitation). (b) Top and side view of chisel-nosed projectile.

The existence of large cracks resulting from cavitation (Fig. 100) signifies that a lot of material expanded away from the projectile nose. This is consistent with Fig. 65, which shows that little ejecta was generated upon penetration. Similar to penetration by chisel-nosed 
projectiles, the penetration channel of RCCs was smaller than its diameter. Lateral expansion was more prevalent for these projectiles because the incoming material had a higher tendency to be redirected around their larger flat nose. We can confirm that the assumption of an ideal plug having a diameter equal to that of the RCC is incorrect and overestimates the axial acceleration of materials, hence the poor correlation to the RI plugging model in Fig. 64. On the other hand, chisel-nosed projectiles were adequately represented by the model since their narrow flat nose decreased the tendency of the material to laterally expand (although still present) and imparted a higher normal pressure onto the target. The latter encouraged proper interfacial contact between projectile nose and target, which reduced the potential cavity formation effect, in Fig. 62.

\subsection{Ballistic Response of Particle-filled Elastomers}

A notable difference in ballistic response was observed as particles were integrated within the elastomer. As seen in Fig. 72, the amount of ejecta increased significantly with particle content and approached a shear plugging response accordingly. Therefore, the target behaviour transitioned from the idealized model in Fig. 62 towards that found in Fig. 71. Similar to the ballistic penetration experiments from Petel et al. [112, 44] concerning dense particle suspensions, a typical plug such as those found in Fig. 30 was not formed. Those plugs were developed in metal plates due to concentrated zones of shear stress. The aluminum alloy failed due to cracks propagating ahead of the projectile through a void nucleation, growth, and coalescence process (similar to Fig. 27) while the pure aluminum had minimal voids and experienced plastic shearing prior to fracture [102]. The particulate composites studied in the present thesis were found to experience a complex stress state upon quasi-static penetration (shear punch) testing. Although this is not directly translatable to the ballistic

behaviour, a combination of compressive, tensile, and shear stress states can be expected, as opposed to the development of shear bands spanning the target thickness. The differences in observed ejecta resulted from deterioration of the composite (particle-matrix debonding) 
ahead of the projectile nose during penetration along with shear and tensile failure at the projectile circumference. The small segments of defeated material ahead of the nose then flowed behind the projectile as it continued to penetrate the capsule. This explains why the majority of ejecta was discharged behind the projectile in Fig. 72.

The penetration channels of several composites were cut and examined through SEM and optical photography, which allowed us to qualitatively describe the penetration event. A cross-sectional SEM image showing the initial penetration stage of 4.7 vol.\% SiC-PDMS impacted by an RCC projectile is provided in Fig. 102a. The primary difference of this penetration channel relative to neat PDMS is the formation of numerous long strands throughout the cavity walls. The image was magnified in Fig. 102b for better visualization. No such strands were present in the penetration channel of neat PDMS, therefore, these can be directly attributed to the inclusion of particles. Similar images for 33.3 vol.\% SiC-PDMS were taken midway through the capsule thickness and are shown in Fig. 102c and 102d. Additional SEM images showing evidence of strand formation are provided for 33.3 vol.\% spherical $\mathrm{Al}_{2} \mathrm{O}_{3}$-PDMS in Appendix C.1, Fig. 111.

It is interesting to note that the failure pattern observed in the SEM images has some distinct similarities to delamination of impacted fiber-reinforced composites and to the discing failure mechanism in metals (e.g., Fig. 23a). Discing failure is described by fractures that occur in several planes parallel to the target plate as the projectile penetrates through the thickness. These planar cracks are formed near the back surface of the target where significant bending can occur. Fractures may be preferentially developed in the planes parallel to the target if these have very low fracture toughness [106]. Woodward [118] found that an aluminum alloy failed by either plugging or discing during penetration, which depended on the orientation of the target relative to the extrusion direction. Since the strands found in Fig. 102 occurred only after particle integration, it is plausible that these fillers may have developed preferential fracture planes for crack propagation, similar to discing failure.

As the projectile impacts the target, the material ahead of the nose is subjected to a 


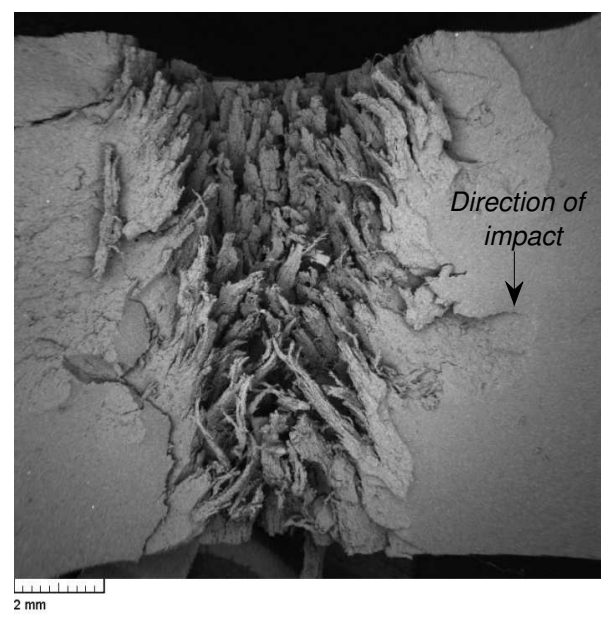

(a)

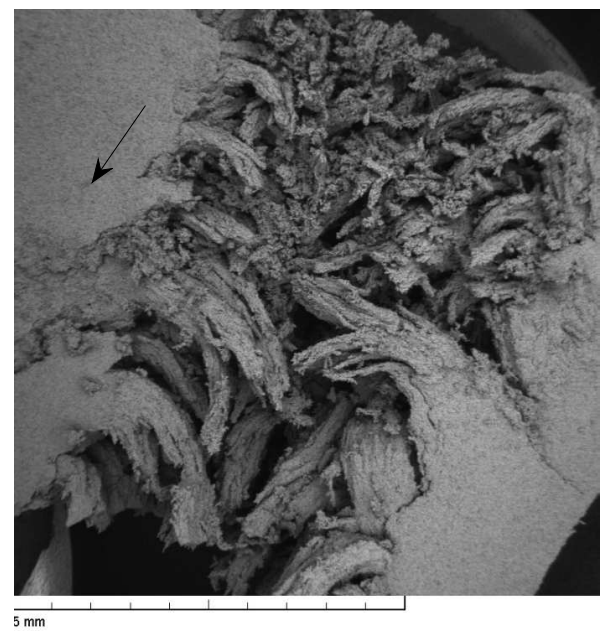

(c)

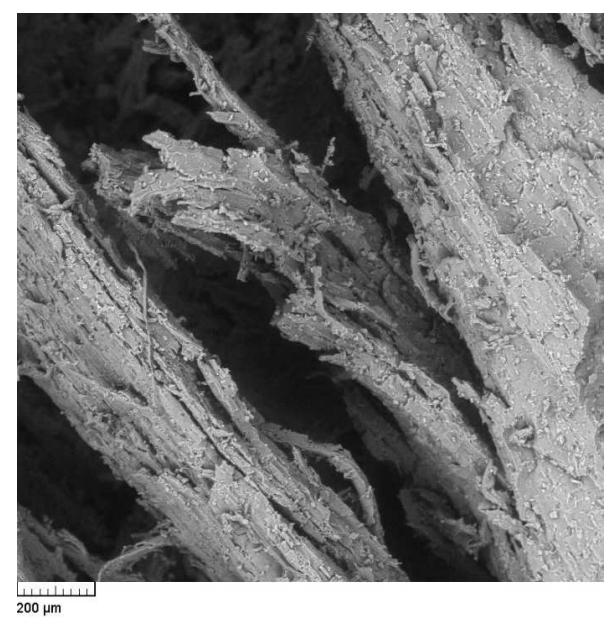

(b)

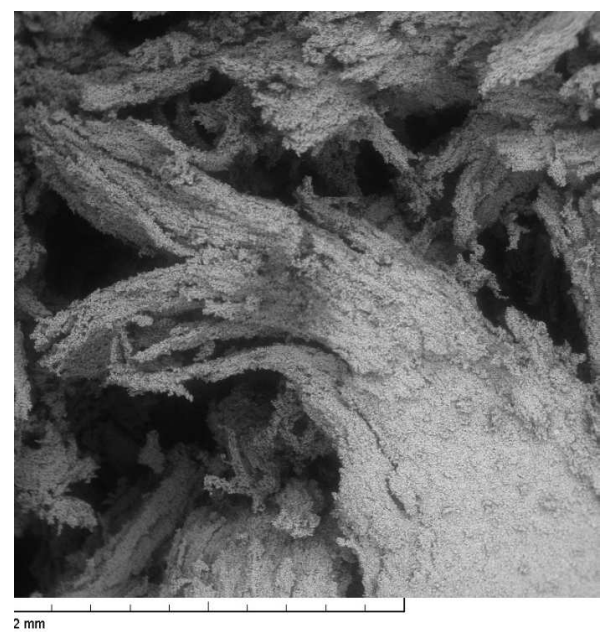

(d)

Figure 102. SEM images showing a portion of penetration channels of SiC-PDMS at different volume fractions (arrows indicate penetration direction): (a) 4.7 vol.\% SiC-PDMS - at impact surface, (b) 4.7 vol.\% SiC-PDMS - strands, (c) 33.3 vol.\% SiC-PDMS - midway through capsule thickness, and (d) 33.3 vol.\% $\mathrm{SiC}$ - strands.

combination of stresses: the material is compressed axially; tensile bending stresses are generated perpendicular to the direction of impact as the material attempts to expand laterally around the projectile nose; and finally, shear stresses result due to bending coupled by shear displacements that occur in the same plane [106]. The composite material surrounding the projectile is in a compressed state and stiffens as it attempts to resist lateral expansion. 
Large deformations at the projectile-target interface induce highly localized shear and tensile stresses at the projectile circumference. These complex stresses may initiate cracks in weak planes that propagate laterally (parallel to the target surfaces) or diagonally outwards, relieving the stresses in the system. Axial cracks may also develop at the projectile circumference. A schematic provided by Woodward [118] illustrates the development of planar cracks through tensile fracture, Fig. 103. Similarly, planar cracks could potentially form ahead of the projectile nose through shear fracture resulting from extensive bending, Fig. 104 [106]. It is however unclear whether it is primarily tensile or shear stresses that are initiating the planar fractures. Regardless, the loosened confinement will decrease the resisting stress exerted by the target as the projectile shears through the material. Although the discing phenomenon typically occurs near the exit surface of metal plates, the planar fractures were found throughout the thickness of our particle-filled elastomers due to the flexibility of the material and the extreme rate of lateral displacement.

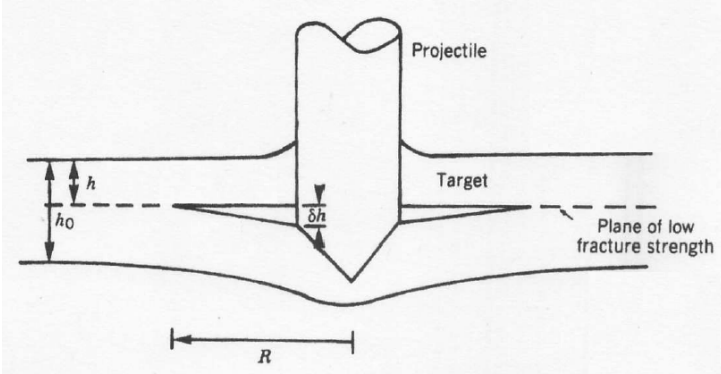

Figure 103. Schematic demonstrating the development of planar cracks through tensile fracture, resulting in discing failure. Figure reproduced with permission from Woodward $[118]$.

Planar cracking (similar to discing) is expected to occur continuously as the projectile penetrates through the target thickness; however, the failure pattern observed in Fig. 102 appear as strands and not a series of fractured planes. As discussed in the previous section, the material tends to expand laterally away from the incoming projectile during penetration, resulting in the formation of radial cracks, e.g., Fig. 105. These occur due to intense hoop stress and are similar to those found in neat PDMS (Fig. 100). Therefore, it is 


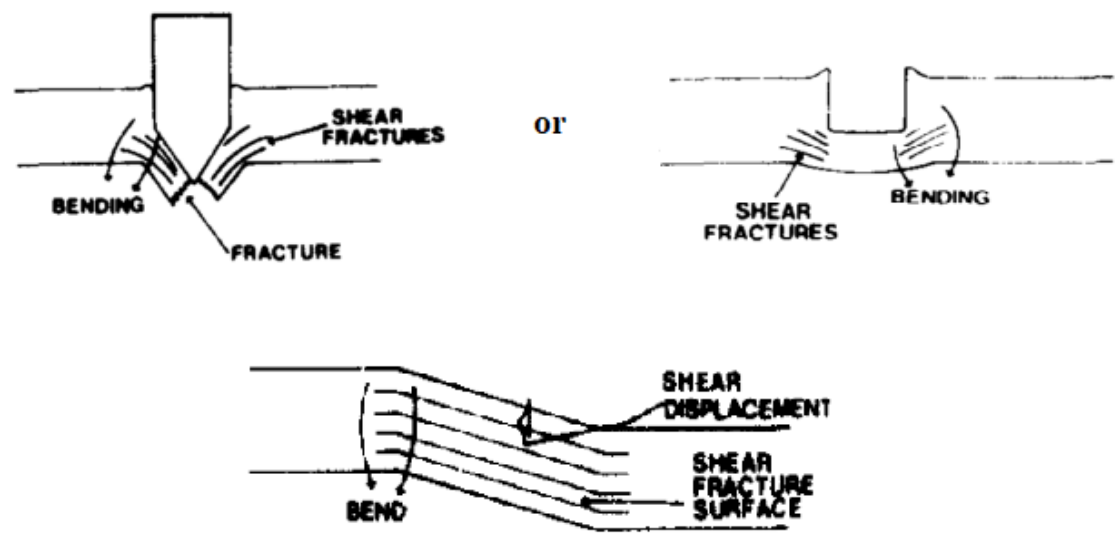

Figure 104. Schematic demonstrating the development of planar cracks through shear fracture, resulting in discing failure. Figure reproduced with permission from Woodward [106].

proposed that the combination of radial and planar cracking is responsible for the formation of strands observed in Fig. 102. A schematic representing the proposed penetration scenario is illustrated in Fig. 106.

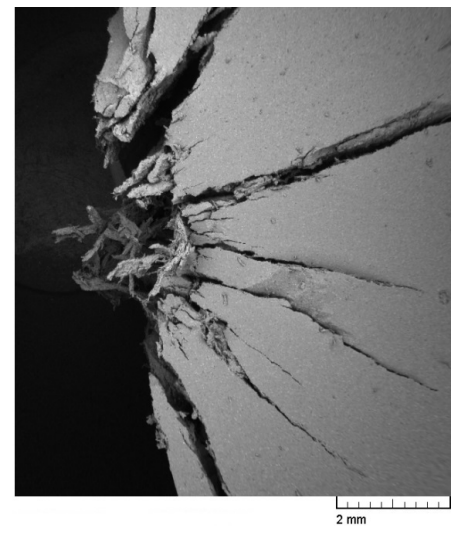

(a)

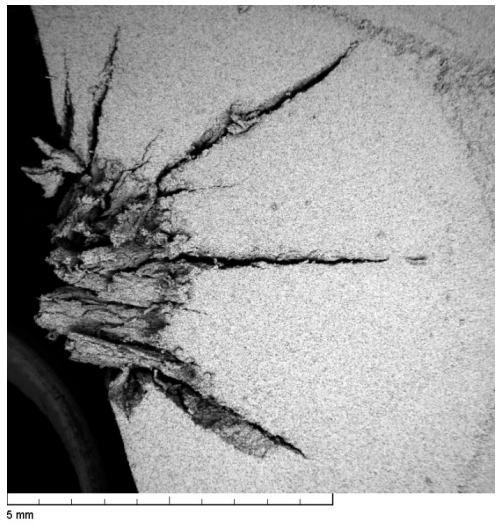

(b)

Figure 105. SEM images showing formation of radial cracks: (a) 4.7 vol.\% SiC-PDMS - impact surface (b) 33.3 vol.\% SiC-PDMS - mid-thickness surface. Penetration direction: into the page.

These observations suggest that particulate fillers can create weak planes (having low fracture toughness) within the elastomer matrix. This phenomenon significantly altered the failure mechanisms relative to neat PDMS. Ballistic capsules have been sectioned into thirds (parallel to impact surface) using a thin cutting blade in order to visualize the progression of 


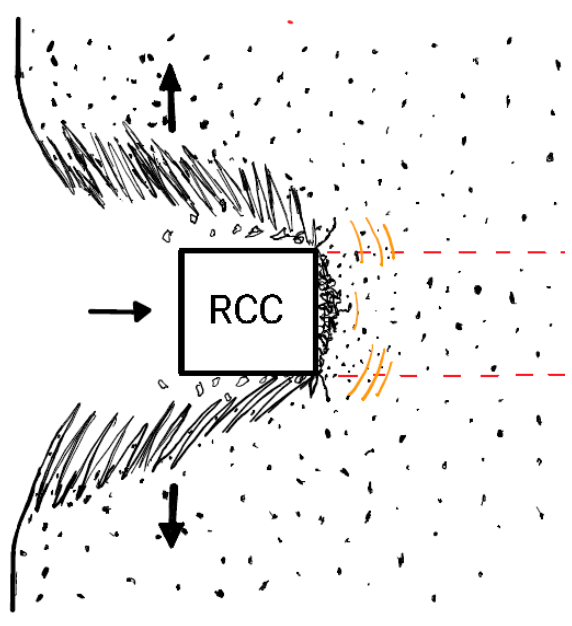

(a)

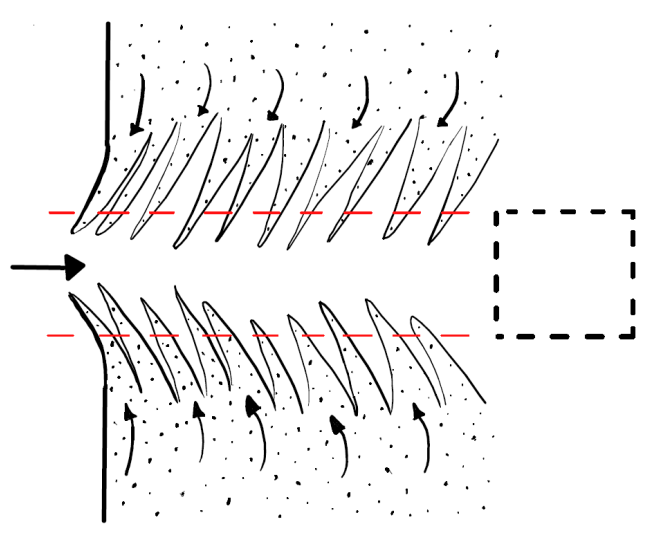

(b)

Figure 106. Schematic illustrating the proposed failure mechanism during penetration of particulate composites: (a) planar tensile fracture may occur at the projectile-target edges while shear fracture may form ahead of nose due to bending (orange lines represent cracks) during penetration; (b) strands return to their original position upon decompression. Arrows indicate direction of motion.

damaged area throughout the target thickness. Photographs of the impact and back (exit) faces along with the surfaces at a relative thickness of $t_{r e l}=0.33$ and 0.67 are provided in Appendix C.2. The sectioned capsule segments were then cut in the plane parallel to the direction of impact to observe differences in the penetration channels (Appendix C.3). These photographs have not been included in the body of the thesis due to the large quantity of images. Capsules were selected in order to visualize the differences in damage that resulted from varying the projectile nose and impact velocity as well as particle volume fraction and material type. The conclusions extracted from the analysis of parallel damaged planes are provided below, followed by a discussion of their corresponding penetration channels.

\subsubsection{Analysis of Through-thickness Damaged Planes}

As discussed in Section 6.5.1, composites consisting of low particle concentrations (4.7 vol.\%) demonstrated a virtually identical ballistic performance compared to neat PDMS when penetrated by both flat-nosed projectiles at intermediate and high velocities. A substantial 
increase in $V_{b l}$ relative to neat PDMS was however observed for composites impacted by RCCs while the opposite was found for chisel-nosed projectiles, Fig. 88. Images of the penetration channel resulting from RCCs impacting neat PDMS showed that a velocitydependent transition in failure mechanism occurred as the projectile approached $V_{b l}$, i.e., initially shearing the material, followed by the axial propagation of a single tensile crack (see Fig. 98). On the other hand, Fig. 97b showed that chisel-nosed projectiles formed a shear plug upon ejection from the back surface.

The damaged surfaces of 4.7 vol.\% SiC-PDMS impacted by RCC and chisel-nosed projectiles slightly above their corresponding $V_{b l}$ values are shown in Appendix C.2, Fig. 112 and 113, respectively. Evidence of shear plugging was noted for the penetration of RCCs up to approximately $t_{r e l}=0.67$. The material then failed due to high tensile bending stresses at the back surface, allowing the projectile to slip through the propagating crack. Chiselnosed projectiles no longer showed signs of extensive shear plugging throughout the capsule thickness and exited the back surface through a tensile crack as well. This differed quite significantly from the penetration of neat PDMS, which clearly demonstrated a plugging event. Therefore, the diminished $V_{b l}$ for 4.7 vol.\% SiC-PDMS impacted by chisel-nosed projectile resulted from a change in failure mechanisms. The addition of particles encouraged tensile failure ahead of the projectile nose instead of shear plugging. This analysis demonstrates the sensitivity of $V_{b l}$ to changes in projectile nose geometry when a low particle content is added to the PDMS.

As the impact velocity increased, 4.7 vol.\% SiC-PDMS targets experienced significant radial cracks throughout their thickness (Appendix C.2, Fig. 114), which resulted from extensive lateral expansion during the penetration of RCCs. Failure at the back surface was found to transition from tensile crack formation to shear plugging. The effect of particle volume fraction can be visualized by comparing the previous photographs with those obtained for 33.3 vol.\% SiC-PDMS in Fig. 115. The damaged area on the impact surface increased with particle content, approaching the diameter of the projectile. Furthermore, 
the lack of radial cracking confirms that the particles assisted in restricting lateral expansion and accelerating material axially, hence the better correlation with the RI plugging model. Radial cracks were formed once again upon further acceleration of RCCs, Fig. 116. Much greater damage was also noted at the back surface of targets, which could be described as a fragmentation failure mechanism (e.g., Fig. 22). Similar images are provided for the 33.3 vol.\% irregular $\mathrm{Al}_{2} \mathrm{O}_{3}$-PDMS in Fig. 117. These images do not provide conclusive evidence for their superior ballistic performance relative to SiC-PDMS, however, it is noted that larger but fewer radial cracks seemed to have formed throughout the thickness. The different damage patterns resulting from penetration of chisel-nosed and hemispherical projectiles are shown in Fig. 118 and 119, respectively.

\subsubsection{Analysis of Ballistic Penetration Channels}

As previously mentioned, the sectioned capsules were cut through the damaged area in order to expose the penetration channel. Photographs of the channels were acquired and stitched together to visualize the damage throughout the entire length of the capsules. The images have been organized in Appendix C.3 in order to observe the differences resulting from variations in particle volume fraction, material, impact velocity, and projectile nose geometry. For example, the penetration channel of 33.3 vol. $\% \mathrm{Al}_{2} \mathrm{O}_{3}$-PDMS impacted slightly below its $V_{b l}$ is shown in Fig. 107. As previously discussed, failure at weak material planes were responsible for the formation of strands at the initial penetration stage. Thicker strands were formed at intermediate penetration depths, prior to the projectile being arrested. This image reveals an important feature of the penetration mechanics. Once planar fractures occur, the resulting strands are prone to folding back or bending out of the way of the incoming projectile instead of being completely sheared off. Although it is assumed that axial momentum

was still transferred to these strands, it was likely quite limited as compared to proper shear plugging. Moreover, the projectile sheared through less material than anticipated, therefore the work expended during penetration is expected to have decreased. The fact that a clear 
hole cannot be observed in most of the damaged surfaces shown in Appendix C.2 is due to the flexibility of these strands during penetration. The bottom section of the capsule in Fig. 107 reveals that the projectile was pushing a plug prior to being arrested. As the capsule plane was cut, the material ahead of the projectile nose was decompressed, hence explaining why the plug is protruding from the surface. Axial cracks resulting from shear failure at the circumference of the projectile were responsible for partial plug formation. These were noted to bow outwards as they propagated through the material, which was likely due to bending stresses.

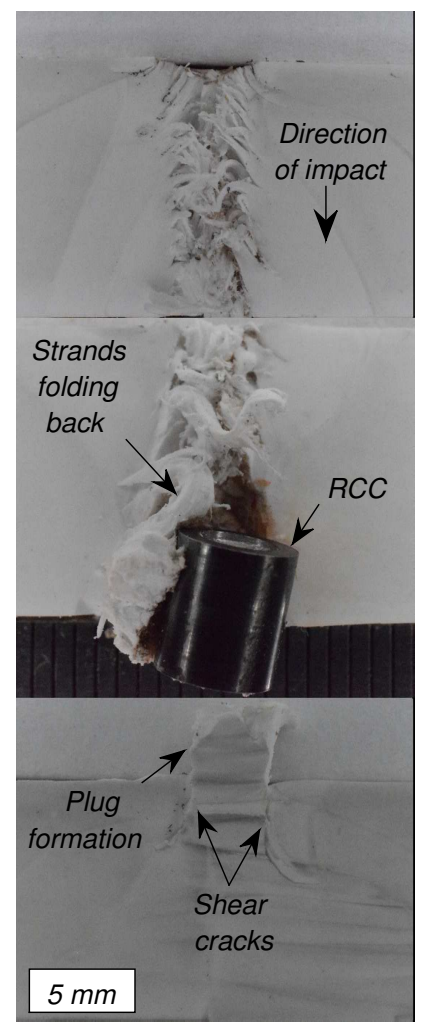

Figure 107. Stitched images showing entire penetration channel of 33.3 vol. $\% \mathrm{Al}_{2} \mathrm{O}_{3}-\mathrm{PDMS}$ impacted slightly below $V_{b l}\left(V_{i}=361 \mathrm{~m} / \mathrm{s}\right)$.

The penetration channels of 4.7 vol.\% SiC-PDMS impacted by RCCs at different $V_{i}$ values are shown in Fig. 120 (Appendix C.3). As the impact velocity increased, the strands formed on the cavity walls became more pronounced. A shearing response was also recovered at the 
back face of the target. These images show that strand formation is partially dependent on projectile velocity. Internal stresses increase with $V_{i}$, which could be inducing more frequent failure at weak material planes (Fig. 103 and 104). It should be noted that upon dissection of the capsule in Fig. 120a, a shear plug was found to have remained trapped within the channel prior to tensile failure at the back face. The plug was removed from the channel and is shown in Fig. 108. One can clearly note the smaller diameter of the plug relative to the RCC (diameter $\approx 5.4 \mathrm{~mm}$ ), which provides evidence of diminished axial acceleration of the target due to lateral expansion effects. This also proves that a solid plug can form during the penetration of particle-filled elastomers, which likely depends on the magnitude of internal stresses and $V_{i}$.

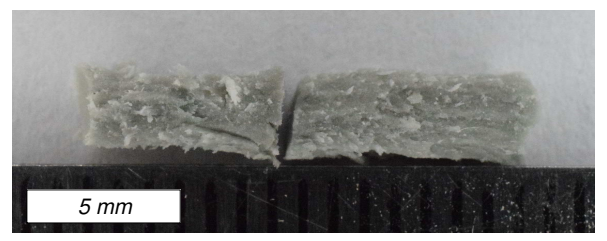

Figure 108. Trapped shear plug that was extracted from 4.7 vol.\% SiC-PDMS capsule impacted by RCC at $V_{i}=257 \mathrm{~m} / \mathrm{s}$. The plug diameter (approximately $2.5 \mathrm{~mm}$ ) was much smaller than that of the projectile $(5.4 \mathrm{~mm})$.

The effect of particle volume fraction is shown for SiC-PDMS impacted by RCCs in Fig. 121. Much finer strands seemed to have formed in 33.3 vol.\% composites during the initial penetration stage, followed by thicker strands at intermediate penetration depths. An increased particle content may be providing more crack initiation zones throughout the target thickness, hence the formation of thinner and more frequent strands. The damaged area seems to have slightly increased with increasing particle content, which is consistent with the notion that particles assist in restricting lateral expansion during penetration. Furthermore, the penetration channel diameter appeared to approach that of the projectile with increasing impact velocity, Fig. 122. The larger internal pressures seem to have increased the frequency of planar fractures, resulting in the formation of finer strands up to intermediate target thicknesses $\left(t_{r e l}=0.67\right)$. As mentioned in the previous section, higher impact velocities also 
induced fragmentation at the back face.

The differences in penetration channels resulting from variations in particle material type are shown in Fig. 123. Similar channel diameters were observed, although it appears that the channel of $\mathrm{Al}_{2} \mathrm{O}_{3}$-PDMS consisted of relatively thicker strands. This may be a result of superior particle-matrix adhesion effects, which was previously suggested as a dominant factor for improved ballistic performance. Strong adhesion would reduce the frequency of planar transverse fracture ahead of the projectile nose and at its periphery, resulting in a larger pressure imposed onto the projectile. Such an effect is expected to manifest itself through the formation of thicker strands within the penetration channel, or the complete absence of strands. Similar fragmentation failure patterns occurred at the back surface of both composites.

Variations in penetration channel characteristics for 33.3 vol.\% SiC-PDMS impacted by different projectile noses are shown in Fig. 124. Less cracks were formed during the penetration of chisel-nosed projectiles, resulting in thicker strands. This may be attributable to the angled surfaces of the projectile nose. A different style of strands was observed for capsules penetrated by hemispherical projectiles, suggesting that different penetration mechanics came to play, as expected.

The present chapter investigated the failure mechanisms associated to the penetration of several elastomeric systems having different microstructures; the conclusions have been summarized below. The dominant effects of lateral expansion were visualized for neat PDMS, as well as the variations in failure patterns resulting from different projectile geometries. The addition of particles within the system resulted in the formation of strands onto the penetration cavity walls, which is analogous to the delamination or discing failure mechanisms. These strands were formed through a combination of planar fracture and radial cracking and are expected to decrease the resistive stress exerted by the target onto the projectile since they are capable of folding back during penetration instead of being sheared off. The proposed penetration event is illustrated in Fig. 106. The damaged surfaces throughout the 
thickness of composite targets were qualitatively analyzed. The differences in $V_{b l}$ values for 4.7 vol.\% composites impacted by RCC and chisel-nosed projectiles were explained using such images. Radial cracking during penetration was impeded by increasing the particle content, i.e., restricting lateral expansion. These cracks were initiated once again at higher $V_{i}$ values. The damaged surface area was found to increase with both particle content and impact velocity, thus, approaching the diameter of the projectile. The fact that resistive shear stresses appeared to increase at higher $V_{i}$ values (Fig. 93) may be partially linked to this varying plug diameter. The analysis of various penetration channels showed that the formation of strands within the cavity walls increased with impact velocity. Fracture planes (transverse cracking) became more frequent at higher particle volume fractions, resulting in finer strands. It appears that improved particle adhesion may have decreased the occurrence of planar fractures, resulting in thicker strands and superior resistive stress. 


\section{Conclusion}

The ballistic response of particle-filled elastomers was investigated to determine the role of particulates and the effects imposed by different microstructure parameters during a penetration event. Ballistic capsules were prepared in order to assess the effect of particle volume fraction, material type, and morphology. Thick targets were penetrated by 16-grain fragment simulating projectiles to record residual velocity data and compare the results to analytical models. Failure mechanisms were inspected through post-mortem images to further understand the behaviour of particulate composites during penetration. The results from ballistic testing were complemented by quasi-static penetration (shear punch) and tensile experiments to compare the resulting trends, identify similarities, and shed light on the contributions provided by particle integration. The conclusions obtained from quasi-static experiments are briefly summarized below, followed by those extracted from ballistic testing.

The tensile strength of PDMS diminished upon particle integration, which implies that composites exhibited poor particle-matrix adhesion characteristics. On the other hand, shear strength was found to increase with particle volume fraction, demonstrating that proper interfacial adhesion was not as critical for this failure mode. Composite stiffness increased with particle content for both loading conditions. Irregular $\mathrm{Al}_{2} \mathrm{O}_{3}$-PDMS composites consistently demonstrated the highest strength and stiffness values for both loading conditions. The surface chemistry of $\mathrm{Al}_{2} \mathrm{O}_{3}$ may have been more compatible with PDMS compared to the other particles, resulting in superior adhesion strength and better overall performance.

The ballistic response of composites impacted by RCC projectiles was thoroughly analyzed and shall be addressed first. The highly elastic nature of neat PDMS induced significant lateral expansion during penetration. Particle integration was found to restrict this lateral motion due to improved material stiffness, therefore promoting axial acceleration of the target. The addition of a low particle content offered negligible improvements in ballistic resistance at intermediate and high impact velocities. The transition towards an ejectadominated response required the particle volume fraction to be sufficiently high in order 
to properly constrain the material ahead of the projectile nose. This was achieved as the particle content increased to 33.3 vol.\%; the behaviour of such composites was adequately represented by the RI plugging model.

The ballistic performance of 33.3 vol.\% particulate composites appeared to be dominated by particle density, whereas variations in particle stiffness and strength did not influence their dynamic response at the volume fractions considered. Such behaviour suggests that extensive interparticle contact (i.e., force chains) did not occur during penetration. The $V_{b l}$ of composites increased with increasing particle density, although an especially large improvement was noted for $\mathrm{Al}_{2} \mathrm{O}_{3}$-PDMS. This composite demonstrated superior particle adhesion during quasi-static testing and so it was assumed that the improved ballistic resistance may have resulted from particle adhesion effects as well. Consequently, $\mathrm{Al}_{2} \mathrm{O}_{3}$-PDMS demonstrated much higher resistive shear stress values compared to other composites.

The interactions between particle and polymer chains were investigated through a morphology study. Irregular-shaped $\mathrm{Al}_{2} \mathrm{O}_{3}$ particles provided superior reinforcement relative to their spherical equivalents under both quasi-static and ballistic conditions. This was primarily attributed to the much larger stress concentration factors provided by irregular (angular) particles and the potential influence arising from their larger aspect ratios. The effect of filler morphology was significantly reduced when substituting the irregular $\mathrm{Al}_{2} \mathrm{O}_{3}$ particles with SiC. Results from the comparison suggest that proper particle adhesion may be crucial in order to observe a significant effect from particle morphology. Radial and transverse fracture planes were generated throughout the thickness of the targets during penetration and were likely caused by weak material interfaces resulting from particle integration. This failure mechanism is analogous to delamination in fiber-reinforced composites or discing in metals. The benefits arising from irregular particles occur when the composite experiences high stresses, however, these cracks act to relieve internal stresses. The loosened confinement of the material limits the ability of irregular particles to improve the ballistic resistance. The superior adhesion strength provided by $\mathrm{Al}_{2} \mathrm{O}_{3}$ over $\mathrm{SiC}$ may have decreased transverse 
fracture, therefore maintaining higher internal stresses ahead of the projectile nose.

Elastomeric systems were found to be especially susceptible to penetration by hemispherical projectiles, resulting in much lower $V_{b l}$ values compared to their flat-nosed counterparts. 33.3 vol.\% particulate composites penetrated by RCC or chisel-nosed projectiles exhibited similar $V_{b l}$ values. The plugging response of chisel-nosed projectiles however consistently exceeded the theoretical range imposed by the RI models, suggesting that the effective plugging area (represented by $A_{r}=0.58$ ) was underestimated. The assumptions employed by the RI plugging model appear to be incorrect for the particulate composites under investigation. Penetration channel diameters approached that of the projectile with increasing particle volume fraction and impact velocity, therefore, it is unwise to assume a constant plug diameter equal to that of the projectile. Furthermore, the penetration work term is likely dependent on impact velocity since the degree of material compression induces an increase in effective particle volume fraction. Shear punch experiments showed that shear strength increases with particle volume fraction; this trend likely translates to high-strain-rate conditions. Once again, these considerations are dependent on the fracture behaviour of targets during penetration. An inertia term that accounts for lateral expansion of the material may be useful to characterize the response of low-particle-content systems.

The present study has provided significant insight concerning the ballistic response of particle-filled elastomeric systems. Several conclusions were suggested, however, some of these still require further validation. The future of this study is directed towards investigating the role of particle surface chemistry by coating the fillers with different coupling agents prior to integration within the elastomer. Numerical simulations of the penetration event would also be advantageous in order to visualize the failure mechanics while considering particlematrix adhesive strength. In addition, efforts to increase the maximum particle volume fraction shall be pursued as an attempt to promote extensive interparticle contact upon impact and reach the full potential of the composite. This could be accomplished by either modifying the manufacturing method or by selecting a different elastomer matrix. 


\section{$9 \quad$ References}

[1] R. Lane, "High Performance Fibers for Personnel and Vehicle Armor Systems," The AMPTIAC Quarterly, vol. 9, no. 2, pp. 3-9, 2005.

[2] M. Übeyli, R. O. Yildirim, and B. Ögel, "On the comparison of the ballistic performance of steel and laminated composite armors," Materials and Design, vol. 28, no. 4, pp. $1257-1262,2007$.

[3] A. Arias, R. Zaera, J. Lopez-Puente, and C. Navarro, "Numerical modeling of the impact behavior of new particulate-loaded composite materials," Composite Structures, vol. 61, no. 1-2, pp. 151-159, 2003.

[4] "Opportunities in protection materials science and technology for future army applications," tech. rep., National Research Council, Washington, DC, 2011.

[5] J. A. Zukas, High Velocity Impact Dynamics. New York: John Wiley and Sons Inc., 1990.

[6] W. Gooch Jr., "An overview of ceramic armor applications," in 6th Technical Conference IDEE 2004, 2004.

[7] E. S. Chin, "Army focused research team on functionally graded armor composites," Materials Science and Engineering A259, vol. 259, pp. 155-161, 1999.

[8] L. A. Utracki, "Rigid ballistic composites (Review of literature)," tech. rep., National Research Council Canada, 2010.

[9] Y. Li and K. Ramesh, "Influence of particle volume fraction, shape, and aspect ratio on the behavior of particle-reinforced metal-matrix composites at high rates of strain," Acta Materialia, vol. 46, no. 16, pp. 5633-5646, 1998. 
[10] S. Yadav and D. R. Chichili, "The mechanical response of a 6061-T6 A1/A12O3 metal matrix composite at high rates of deformation," Acta metall. mater., vol. 43, no. 12, pp. 4453-4464, 1995.

[11] M. B. Karamis, A. Tasdemirci, and F. Nair, "Failure and tribological behaviour of the AA5083 and AA6063 composites reinforced by SiC particles under ballistic impact," Composites Part A: Applied Science and Manufacturing, vol. 34, no. 3, pp. 217-226, 2003.

[12] S. Ozden, R. Ekici, and F. Nair, "Investigation of impact behaviour of aluminium based $\mathrm{SiC}$ particle reinforced metal-matrix composites," Composites Part A: Applied Science and Manufacturing, vol. 38, no. 2, pp. 484-494, 2007.

[13] W. D. Callister and D. G. Rethwisch, Materials science and engineering: An introduction. John Wiley \& Sons Inc., 8 ed., 2010.

[14] S.-M. Choi and H. Awaji, "Nanocomposites - a new material design concept," Science and Technology of Advanced Materials, vol. 6, no. 1, pp. 2-10, 2005.

[15] S. Y. Fu, X. Q. Feng, B. Lauke, and Y. W. Mai, "Effects of particle size, particle/matrix interface adhesion and particle loading on mechanical properties of particulate-polymer composites," Composites Part B: Engineering, vol. 39, no. 6, pp. 933-961, 2008.

[16] U. Maitra, K. E. Prasad, U. Ramamurty, and C. Rao, "Mechanical properties of nanodiamond-reinforced polymer-matrix composites," Solid State Communications, vol. 149, no. 39-40, pp. 1693-1697, 2009.

[17] I. Neitzel, V. Mochalin, I. Knoke, G. R. Palmese, and Y. Gogotsi, "Mechanical properties of epoxy composites with high contents of nanodiamond," Composites Science and Technology, vol. 71, no. 5, pp. 710-716, 2011. 
[18] Y. S. Thio, A. S. Argon, and R. E. Cohen, "Role of interfacial adhesion strength on toughening polypropylene with rigid particles," Polymer, vol. 45, no. 10, pp. 31393147, 2004.

[19] R. Kitey and H. V. Tippur, "Role of particle size and filler-matrix adhesion on dynamic fracture of glass-filled epoxy. I. Macromeasurements," Acta Materialia, vol. 53, no. 4, pp. 1153-1165, 2005.

[20] V. Kushvaha and H. Tippur, "Effect of filler shape, volume fraction and loading rate on dynamic fracture behavior of glass-filled epoxy," Composites Part B: Engineering, vol. 64, pp. 126-137, 2014.

[21] D. E. Munson, R. R. Boade, and K. W. Schuler, "Stress-wave propagation in Al2O3 -epoxy mixtures," Journal of Applied Physics, vol. 49, no. 9, pp. 4797-4807, 1978.

[22] R. E. Setchell and M. U. Anderson, "Shock-compression response of an alumina-filled epoxy," Journal of Applied Physics, vol. 97, no. 8, 2005.

[23] T. J. Vogler, C. S. Alexander, J. L. Wise, and S. T. Montgomery, "Dynamic behavior of tungsten carbide and alumina filled epoxy composites," Journal of Applied Physics, vol. 107, no. 4, 2010.

[24] J. Jordan, J. Spowart, M. Kendall, B. Woodworth, and C. Siviour, "Mechanics of particulate composites with glassy polymer binders in compression.," Phil. Trans. R. Soc. A, vol. 372, no. 20130215, 2014.

[25] Z. Petrovic and W. Zhang, "Glassy And Elastomeric Polyurethanes Filled With NanoSilica Particles," Materials Science Forum, vol. 352, pp. 171-176, 2000.

[26] M. J. N. Jacobs and J. L. J. Van Dingenen, "Ballistic protection mechanisms in personal armour," Journal of Materials Science, vol. 36, no. 13, pp. 3137-3142, 2001. 
[27] N. Naik, P. Shrirao, and B. Reddy, "Ballistic impact behaviour of woven fabric composites: Formulation," Journal of Impact Engineering, vol. 32, pp. 1521-1552, 2006.

[28] R. Mines, A. Roach, and N. Jones, "High Velocity Perforation Behaviour of Polymer Composite Laminates," International Journal of Impact Engineering, vol. 22, pp. 561$588,1999$.

[29] S. K. García-Castillo, S. Sánchez-Sáez, and E. Barbero, "Nondimensional analysis of ballistic impact on thin woven laminate plates," International Journal of Impact Engineering, vol. 39, no. 1, pp. 8-15, 2012.

[30] B. L. Buitrago, S. K. García-Castillo, and E. Barbero, "Influence of shear plugging in the energy absorbed by thin carbon-fibre laminates subjected to high-velocity impacts," Composites Part B: Engineering, vol. 49, pp. 86-92, 2013.

[31] N. Nayak, P. Sivaraman, A. Banerjee, V. Madhu, A. Dutta, V. Mishra, and B. Chakraborty, "Effect of Matrix on the Ballistic Impact of Aramid Fabric Composite Laminates by Armor Piercing Projectiles," Polymer Composites, pp. 444-450, 2012.

[32] B. Cheeseman and T. Bogetti, "Ballistic impact into fabric and compliant composite laminates," Composite Structures, vol. 61, no. 1-2, pp. 161-173, 2003.

[33] D. Roylance, A. Wilde, and G. Tocci, "Ballistic Impact of Textile Structures," Textile Research Journal, vol. 43, no. 1, pp. 34-41, 1973.

[34] X. S. Zeng, V. B. C. Tan, and V. P. W. Shim, "Modelling inter-yarn friction in woven fabric armour," International Journal for Numerical Methods in Engineering, vol. 66, no. 8, pp. 1309-1330, 2006.

[35] Z. Dong, J. Manimala, and C. T. Sun, "Mechanical behavior of silica nanoparticle- 
impregnated kevlar fabrics," Journal of Mechanics of Materials and Structures, vol. 5, no. 4, pp. 529-548, 2010.

[36] B. Lee, T. Walsh, S. Won, H. Patts, J. Song, and A. Mayer, "Penetration Failure Mechanisms of Armor-Grade Fiber Composites under Impact," Journal of Composite Materials, vol. 35, no. 18, pp. 1605-1633, 2001.

[37] L. Gates Jr., "Evaluation and Development of Fluid Armor Systems," tech. rep., Air Force Materials Laboratory, 1968.

[38] L. Gates Jr., "Flexible protective armour material and method of making same," 1972.

[39] O. E. Petel, D. Frost, A. Higgins, and S. Ouellet, "Lateral Stress Measurements in Dense Suspensions," in AIP Conference Proceedings, vol. 1426, pp. 1495-1498, 2012.

[40] Y. Lee, E. Wetzel, and N. Wagner, "The Ballistic Impact Characteristics of Kevlar Woven Fabrics Impregnated with a Colloidal Shear Thickening Fluid," Journal of Materials Science, vol. 38, pp. 2825-2833, 2003.

[41] R. Egres, M. Decker, C. Halbach, Y. Lee, J. Kirkwood, K. Kirkwood, N. Wagner, and E. Wetzel, "Stab Resistance of STF - Kevlar Composites for Body Armor Applications," in 24th Army Science Conference, vol. 36, pp. 30-35, 2004.

[42] R. Egres Jr., C. Halbach, M. Decker, E. Wetzel, and N. Wagner, "Stab Performance of Shear Thickening Fluid (STF)-Fabric Composites for Body Armor Applications," in SAMPE 2005: New Horizons for Materials and Processing Technologies, (Long Beach, CA), 2005.

[43] A. Srivastava, A. Majumdar, and B. S. Butola, "Improving the Impact Resistance of Textile Structures by using Shear Thickening Fluids: A Review," Critical Reviews in Solid State and Materials Sciences, vol. 37, no. 2, pp. 115-129, 2012. 
[44] O. E. Petel, S. Ouellet, J. Loiseau, D. L. Frost, and A. J. Higgins, "A comparison of the ballistic performance of shear thickening fluids based on particle strength and volume fraction," International Journal of Impact Engineering, vol. 85, pp. 83-96, 2015.

[45] D. P. Kalman, R. L. Merrill, N. J. Wagner, and E. D. Wetzel, "Effect of particle hardness on the penetration behavior of fabrics intercalated with dry particles and concentrated particle-fluid suspensions," ACS Applied Materials and Interfaces, vol. 1, no. 11, pp. 2602-2612, 2009.

[46] O. E. Petel and A. J. Higgins, "Shock wave propagation in dense particle suspensions," Journal of Applied Physics, vol. 108, no. 114918, 2010.

[47] O. E. Petel, D. L. Frost, A. J. Higgins, and S. Ouellet, "Shock-induced Formation of a Disordered Solid from a Dense Particle Suspension," in AIP Conference Proceedings, vol. 1426, pp. 1459-1462, 2012.

[48] L. Dischler, T. T. Moyer, and J. B. Henson, "Dilatant powder coated fabric and containment articles formed therefrom," 1998.

[49] Y. Duan, M. Keefe, T. Bogetti, and B. Cheeseman, "Modeling friction effects on the ballistic impact behavior of a single-ply high-strength fabric," International Journal of Impact Engineering, vol. 31, no. 8, pp. 996-1012, 2005.

[50] Y. Duan, M. Keefe, T. Bogetti, B. Cheeseman, and B. Powers, "A numerical investigation of the influence of friction on energy absorption by a high-strength fabric subjected to ballistic impact," International Journal of Impact Engineering, vol. 32, no. 8, pp. 1299-1312, 2006.

[51] H. M. Wen, "Penetration and perforation of thick FRP laminates," Composites Science and Technology, vol. 61, no. 8, pp. 1163-1172, 2001. 
[52] N. McCrum, C. Buckley, and C. Buckmall, Principles of Polymer Engineering. New York: Oxford University Press Inc., 2 ed., 1997.

[53] I. Ward and J. Sweeney, Mechanical properties of solid polymers. Wiley, 3 ed., 2013.

[54] D. Bigg, D. Hiscock, J. Preston, and E. Bradbury, "Performance thermoplastic matrix composites," Journal of Thermoplastic Composite Materials, vol. 1, pp. 146-160, 1988.

[55] J. Karger-Kocsis and S. Fakirov, Nano- and Micro- Mechanics of Polymer Blends and Composites. Hanser, 2009.

[56] F. Schneider, T. Fellner, J. Wilde, and U. Wallrabe, "Mechanical properties of silicones for MEMS," Journal of Micromechanics and Microengineering, vol. 18, no. 6, 2008.

[57] F. Schneider, J. Draheim, R. Kamberger, and U. Wallrabe, "Process and material properties of polydimethylsiloxane (PDMS) for Optical MEMS," Sensors and Actuators, A: Physical, vol. 151, no. 2, pp. 95-99, 2009.

[58] I. Johnston, D. McCluskey, C. Tan, and M. Tracey, "Mechanical characterization of bulk Sylgard 184 for microfluidics and microengineering," Journal of Micromechanics and Microengineering, vol. 24, no. 3, 2014.

[59] V. Studer, G. Hang, A. Pandolfi, M. Ortiz, W. F. Anderson, and S. R. Quake, "Scaling properties of a low-actuation pressure microfluidic valve," Journal of Applied Physics, vol. 95, no. 1, pp. 393-398, 2004.

[60] J. Friend and L. Yeo, "Fabrication of Microfluidic Devices using Polydimethylsiloxane," Journal of Biomicrofluidics, vol. 4, 2010.

[61] K. M. Choi and J. A. Rogers, "A photocurable poly(dimethylsiloxane) chemistry designed for soft lithographic molding and printing in the nanometer regime," Journal of the American Chemical Society, vol. 125, no. 14, pp. 4060-4061, 2003. 
[62] "Dow Corning Product Information Sylgard @ 184 Silicone Elastomer," 2013.

[63] T. K. Kim, J. K. Kim, and O. C. Jeong, "Measurement of nonlinear mechanical properties of PDMS elastomer," Microelectronic Engineering, vol. 88, no. 8, pp. 1982-1985, 2011.

[64] J. C. Millett, G. Whiteman, S. M. Stirk, and N. K. Bourne, "Shear strength measurements in a shock loaded commercial silastomer," Journal of Physics D: Applied Physics, vol. 44, no. 18, 2011.

[65] S. Marsh, LASL shock Hugoniot data. Los Angeles: University of California Press, 1980.

[66] R. E. Winter, G. Whiteman, G. S. Haining, D. A. Salisbury, and K. Tsembelis, "Measurement of Equation of State of Silicone Elastomer," in AIP Conference Proceedings, vol. 706, pp. 679-684, 2004.

[67] T. Hirsch, "Modulus of elasticity of concrete affected by elastic moduli of cement paste matrix and aggregate," J. Amer. Conc. Inst., vol. 59, no. 3, pp. 427-452, 1962.

[68] S. Ahmed and F. R. Jones, "A review of particulate reinforcement theories for polymer composites," Journal of Materials Science, vol. 25, no. 12, pp. 4933-4942, 1990.

[69] M. Folkes, Short fiber reinforced thermoplastics. New York: John Wiley \& Sons Inc., 1982.

[70] D. Bigg, "Mechanical properties of particulate filled polymers," Polymer Composites, vol. 8, no. 2, pp. 115-122, 1987.

[71] E. Guth, "Theory of filler reinforcement," Journal of Applied Physics, vol. 16, no. 1, pp. 20-25, 1945. 
[72] L. Nicolais and M. Narkis, "Stress-strain Behavior of Styrene-Acrylonitrile/Glass Bead Composites in the Glassy Region," Polymer Engineering 85 Science, vol. 11, no. 3, pp. 194-199, 1971.

[73] L. Nicolais and L. Nicodemo, "Strength of particulate composite," Polymer Engineering and Science, vol. 13, no. 6, p. 469, 1973.

[74] J. Leidner and R. Woodhams, "The Strength of Polymeric Composites Containing Spherical Fillers," Journal of Applied Polymer Science, vol. 18, pp. 1639-1654, 1974.

[75] P. Cooper, "Shock waves," in Explosives Engineering, ch. 14-19, Wiley-VCH, 1996.

[76] J. Jordan, E. B. Herbold, G. Sutherland, A. Fraser, J. Borg, and D. Richards, "Shock equation of state of multi- constituent epoxy-metal particulate composites," Journal of Applied Physics, vol. 109, 2011.

[77] J. Jordan and J. Spowart, "Comparison of Mechanical Properties of Polymer-Based Multi-phase Particulate Composites," in Dynamic Behavior of Materials: Conference Proceedings of the Society for Experimental Mechanics Series, vol. 1, pp. 317-320, 2013.

[78] O. E. Petel, D. L. Frost, A. J. Higgins, and S. Ouellet, "Shock-induced formation of a disordered solid from a dense particle suspension," Physical Review E, vol. 85, 2012.

[79] B. J. Marr, O. E. Petel, D. L. Frost, A. J. Higgins, and S. Ringuette, "Shock-induced deformation in wetted particle beds," Journal of Physics: Conference Series, vol. 500, no. 11, 2014.

[80] G. Weirauch, Das Verhalten von Kupferstiften beim Auftreffen auf verschiedene Werkstoffe mit Geschwindigkeiten zwischen $50 \mathrm{~m} / \mathrm{s}$ und $1650 \mathrm{~m} / \mathrm{s}$. Ph.d. dissertation, University of Karlsruhe, 1971.

[81] Z. Rosenberg and E. Dekel, Terminal Ballistics. Springer, 2013. 
[82] M. E. Backman and W. Goldsmith, "The mechanics of penetration of projectiles into targets," International Journal of Engineering Science, vol. 16, no. 1, pp. 1-99, 1978.

[83] G. G. Corbett, S. R. Reid, and W. Johnson, "Impact loading of plates and shells by free-flying projectiles: A review," International Journal of Impact Engineering, vol. 18, no. 2, pp. 141-230, 1996.

[84] A. Piekutowski, M. Forrestal, K. Poormon, and T. Warren, "Penetration of 6061-T6511 Aluminum Targets by Ogive-nose Steel Projectiles with Stricking Velocities Between 0.5 and $3.0 \mathrm{~km} / \mathrm{s}$," International Journal of Impact Engineering, vol. 23, pp. 723-734, 1999.

[85] M. Forrestal, V. Luk, Z. Rosenberg, and N. Brar, "Penetration of 7075-T651 aluminum targets with ogival-nose rods," International Journal of Solids and Structures, vol. 29, no. 14-15, pp. 1729-1736, 1992.

[86] Z. Rosenberg and E. Dekel, "The penetration of rigid long rods - revisited," International Journal of Impact Engineering, vol. 36, no. 4, pp. 551-564, 2009.

[87] R. Hill, "Cavitation and the Influence of Headshape in Attack of Thick Targets by Non-deforming Projectiles," J. Mech. Phys. Solids, vol. 28, pp. 249-263, 1980.

[88] Z. Rosenberg and E. Dekel, "A numerical study of the cavity expansion process and its application to long-rod penetration mechanics," International Journal of Impact Engineering, vol. 35, no. 3, pp. 147-154, 2008.

[89] Z. Rosenberg and E. Dekel, "On the deep penetration and plate perforation by rigid projectiles," International Journal of Solids and Structures, vol. 46, no. 24, pp. 41694180, 2009.

[90] K. Senthil, M. Iqbal, B. Arindam, R. Mittal, and N. Gupta, "Ballistic resistance of 
2024 aluminium plates against hemispherical, sphere and blunt nose projectiles," ThinWalled Structures, pp. 1-12, 2017.

[91] A. Arias, J. A. Rodríguez-Martínez, and A. Rusinek, "Numerical simulations of impact behaviour of thin steel plates subjected to cylindrical, conical and hemispherical nondeformable projectiles," Engineering Fracture Mechanics, vol. 75, no. 6, pp. 1635-1656, 2008.

[92] T. Borvik, M. Langseth, O. S. Hopperstad, and K. A. Malo, "Perforation of 12mm thick steel plates by $20 \mathrm{~mm}$ diameter projectiles with flat, hemispherical and conical noses - Part I: Experimental study," International Journal of Impact Engineering, vol. 27, no. 1, pp. 19-35, 2002.

[93] K. M. Kpenyigba, T. Jankowiak, A. Rusinek, R. Pesci, and B. Wang, "Effect of projectile nose shape on ballistic resistance of interstitial-free steel sheets," International Journal of Impact Engineering, vol. 79, pp. 83-94, 2015.

[94] A. Husain, R. Ansari, and A. H. Khan, "Experimental and numerical investigation of perforation of thin polycarbonate plate by projectiles of different nose shape," Latin American Journal of Solids and Structures, vol. 14, no. 2, pp. 357-372, 2017.

[95] I. Mohagheghian, G. J. McShane, and W. J. Stronge, "Impact perforation of monolithic polyethylene plates: Projectile nose shape dependence," International Journal of Impact Engineering, vol. 80, pp. 162-176, 2015.

[96] A. L. Wingrove, "The influence of projectile geometry on adiabatic shear and target failure," Metallurgical Transactions, vol. 4, no. 8, pp. 1829-1833, 1973.

[97] R. Recht and T. Ipson, "Ballistic Perforation Dynamics," Journal of Applied Mechanics, vol. 30, pp. 384-390, 1963. 
[98] R. Woodward, "The Penetration of Metal Targets by Conical Projectiles," International Journal of Mechanical Sciences, vol. 20, pp. 349-359, 1978.

[99] Z. Rosenberg and E. Dekel, "Revisiting the perforation of ductile plates by sharp-nosed rigid projectiles," International Journal of Solids and Structures, vol. 47, no. 22-23, pp. 3022-3033, 2010.

[100] A. Zaid and F. Travis, "An Examination of the effect of target thickness in the perforation of mild steel plate by a flat-ended cylindirical projectile," International Journal of Mechanical Sciences, vol. 16, pp. 373-383, 1974.

[101] T. Borvik, M. Langseth, O. S. Hopperstad, and K. A. Malo, "Ballistic penetration of steel plates," International Journal of Impact Engineering, vol. 22, no. 9, pp. 855-886, 1999.

[102] R. Woodward, "Void formation in defeated aluminum alloy targets," Metallurgical Transactions A, vol. 7, no. 5, pp. 894-896, 1976.

[103] C. Zener and J. H. Hollomon, "Effect of strain rate upon plastic flow of steel," Journal of Applied Physics, vol. 15, no. 1, pp. 22-32, 1944.

[104] C. Zener, "The micro-mechanism of fracture," in Fracturing of metals, pp. 3-31, ASM, 1948.

[105] R. Recht, "Catastrophic Thermoplastic Shear," Journal of Applied Mechanics, vol. 31, no. 2 , pp. 189-193, 1964.

[106] R. Woodward, "The interrelation of failure modes observed in the penetration of metallic targets," International Journal of Impact Engineering, vol. 2, no. 2, pp. 121-129, 1984.

[107] R. Woodward, "The penetration of metal targets which fail by adiabatic shear plugging," International Journal of Mechanical Sciences, vol. 20, no. 9, pp. 599-607, 1978. 
[108] R. Woodward and M. E. de Morton, "Penetration of targets by flat-ended projectiles," International Journal of Mechanical Sciences, vol. 18, no. 3, pp. 119-127, 1976.

[109] A. Rusinek, J. A. Rodríguez-Martínez, R. Zaera, J. R. Klepaczko, A. Arias, and C. Sauvelet, "Experimental and numerical study on the perforation process of mild steel sheets subjected to perpendicular impact by hemispherical projectiles," International Journal of Impact Engineering, vol. 36, no. 4, pp. 565-587, 2009.

[110] K. M. Kpenyigba, T. Jankowiak, A. Rusinek, and R. Pesci, "Influence of projectile shape on dynamic behavior of steel sheet subjected to impact and perforation," ThinWalled Structures, vol. 65, pp. 93-104, 2013.

[111] B. Landkof and W. Goldsmith, "Petalling of thin, metallic plates during penetration by cylindro-conical projectiles," International Journal of Solids and Structures, vol. 21, no. 3, pp. 245-266, 1985.

[112] O. E. Petel, S. Ouellet, J. Loiseau, B. Marr, D. L. Frost, and A. J. Higgins, "The effect of particle strength on the ballistic resistance of shear thickening fluids," Applied Physics Letters, vol. 102, no. 6, 2013.

[113] O. E. Petel and S. Ouellet, "Dynamic strength, particle deformation, and fracture within fluids with impact-activated microstructures," Journal of Applied Physics, vol. 122 , no. 2, 2017.

[114] J. Shackelford and W. Alexander, Materials Science and Engineering Handbook. CRC Press LLC, 3 ed., 2001.

[115] "ASTM D732-10: Shear Strength of Plastics by Punch Tool," ASTM International, 2013.

[116] R. K. Guduru, K. A. Darling, R. Kishore, R. O. Scattergood, C. C. Koch, and K. L. 
Murty, "Evaluation of mechanical properties using shear-punch testing," Materials Science and Engineering A, vol. 395, no. 1-2, pp. 307-314, 2005.

[117] "ASTM D412-06a: Vulcanized Rubber and Thermoplastic Elastomers-Tension," ASTM International, 2006.

[118] R. Woodward, "Penetration Behaviour of a High-strength Aluminium Alloy," Metals Technology, vol. 6, no. 1, pp. 106-110, 1979. 


\section{Appendices}

\section{A Ballistic Capsule Testing Results}

Ballistic penetration experiments were conducted at DRDC Valcartier with the assistance and guidance of their staff. The entire collection of $V_{r}-V_{i}$ data can be found in Table 8 below. 
Table 8. Master ballistic capsule data sheet.

\begin{tabular}{|c|c|c|c|c|c|c|c|}
\hline Material & $\begin{array}{l}\text { Projectile } \\
\text { Nose } \\
\text { Shape }\end{array}$ & $\begin{array}{l}\text { Incident } \\
\text { Velocity } \\
(\mathrm{m} / \mathrm{s})\end{array}$ & $\begin{array}{l}\text { Residual } \\
\text { Velocity } \\
(\mathrm{m} / \mathrm{s})\end{array}$ & $\begin{array}{l}\text { Capsule } \\
\text { Length } \\
(\mathrm{mm})\end{array}$ & $\begin{array}{l}\text { Exp. } \\
\text { Density } \\
(\mathrm{g} / \mathrm{cc})\end{array}$ & $\begin{array}{l}\text { Theo. } \\
\text { Density } \\
\text { (g/cc) }\end{array}$ & Notes \\
\hline \multirow[t]{15}{*}{ Syl 184} & $\mathrm{RCC}$ & 214 & 32 & 38.09 & 1.017 & 1.030 & \\
\hline & $\mathrm{RCC}$ & 300 & 144 & 37.50 & 1.038 & & \\
\hline & $\mathrm{RCC}$ & 345 & 188 & 37.56 & 1.033 & & \\
\hline & $\mathrm{RCC}$ & 435 & 255 & 37.10 & 1.044 & & \\
\hline & $\mathrm{RCC}$ & 522 & 319 & 37.17 & 1.039 & & \\
\hline & $\mathrm{RCC}$ & 613 & 377 & 37.50 & 1.034 & & \\
\hline & $\mathrm{RCC}$ & 702 & 431 & 37.65 & 1.032 & & \\
\hline & $\mathrm{RCC}$ & 888 & 542 & 37.64 & 1.023 & & \\
\hline & conical & 211 & 60 & 37.62 & 1.026 & & Slight projectile yaw \\
\hline & conical & 265 & 103 & 37.83 & 1.026 & & Projectile yaw \\
\hline & conical & 298 & 173 & 37.91 & 1.028 & & \\
\hline & conical & 360 & 223 & 37.85 & 1.026 & & Projectile yaw \\
\hline & conical & 496 & 338 & 37.92 & 1.022 & & \\
\hline & chisel & 216 & 0 & 37.91 & 1.022 & & \\
\hline & chisel & 242 & 43 & 37.89 & 1.023 & & \\
\hline
\end{tabular}




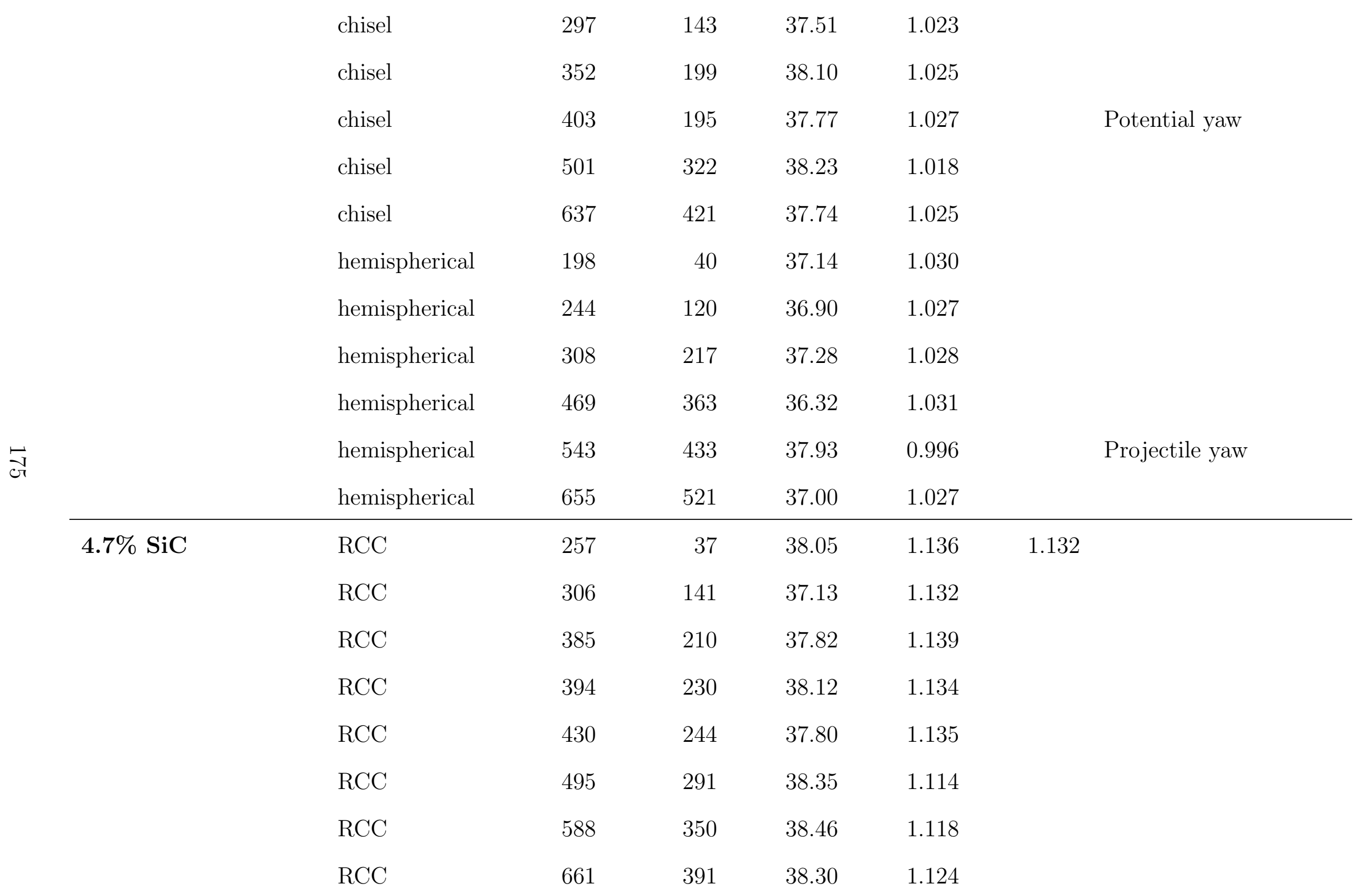




\begin{tabular}{|c|c|c|c|c|c|c|c|}
\hline & $\mathrm{RCC}$ & 873 & 509 & 37.67 & 1.138 & & \multirow{6}{*}{$\begin{array}{l}\text { Slight projectile yaw } \\
\text { Projectile yaw }\end{array}$} \\
\hline & chisel & 229 & 73 & 37.28 & 1.132 & & \\
\hline & chisel & 261 & 115 & 36.88 & 1.132 & & \\
\hline & chisel & 316 & 162 & 37.42 & 1.132 & & \\
\hline & chisel & 483 & 305 & 36.95 & 1.132 & & \\
\hline & chisel & 648 & 418 & 37.29 & 1.132 & & \\
\hline \multirow[t]{12}{*}{$23.6 \% \mathrm{SiC}$} & $\mathrm{RCC}$ & 307 & 84 & 37.60 & 1.550 & \multirow[t]{12}{*}{1.544} & \\
\hline & $\mathrm{RCC}$ & 354 & 99 & 38.12 & 1.548 & & \\
\hline & $\mathrm{RCC}$ & 354 & 120 & 38.20 & 1.553 & & \\
\hline & $\mathrm{RCC}$ & 458 & 182 & 37.90 & 1.547 & & \\
\hline & $\mathrm{RCC}$ & 569 & 258 & 37.76 & 1.547 & & \\
\hline & $\mathrm{RCC}$ & 741 & 335 & 38.26 & 1.555 & & \\
\hline & $\mathrm{RCC}$ & 894 & 418 & 38.33 & 1.545 & & \\
\hline & chisel & 271 & 0 & 37.73 & 1.526 & & \\
\hline & chisel & 309 & 85 & 37.87 & 1.537 & & \\
\hline & chisel & 406 & 170 & 38.10 & 1.532 & & \\
\hline & chisel & 519 & 255 & 38.31 & 1.526 & & \\
\hline & chisel & 710 & 376 & 38.01 & 1.533 & & \\
\hline 33.3\% SiC & $\mathrm{RCC}$ & 350 & 0 & 37.87 & 1.761 & 1.756 & \\
\hline
\end{tabular}




\begin{tabular}{|c|c|c|c|c|c|c|}
\hline & $\mathrm{RCC}$ & 324 & 4 & 38.20 & 1.757 & \\
\hline & $\mathrm{RCC}$ & 423 & 117 & 38.27 & 1.753 & \\
\hline & $\mathrm{RCC}$ & 559 & 210 & 37.75 & 1.740 & \\
\hline & $\mathrm{RCC}$ & 740 & 276 & 38.15 & 1.751 & \\
\hline & $\mathrm{RCC}$ & 885 & 326 & 38.38 & 1.749 & \\
\hline & conical & 186 & 0 & 37.40 & 1.752 & \\
\hline & conical & 295 & 0 & 38.65 & 1.730 & \\
\hline & conical & 359 & 55 & 38.20 & 1.741 & Projectile yaw \\
\hline & conical & 364 & 86 & 38.25 & 1.736 & \\
\hline & conical & 455 & 209 & 37.35 & 1.754 & Projectile yaw \\
\hline & conical & 494 & 204 & 38.56 & 1.740 & \\
\hline & conical & 560 & 268 & 37.40 & 1.751 & \\
\hline & chisel & 316 & 0 & 38.03 & 1.727 & \\
\hline & chisel & 350 & 60 & 37.78 & 1.748 & \\
\hline & chisel & 392 & 108 & 37.26 & 1.762 & \\
\hline & chisel & 479 & 175 & 38.39 & 1.737 & \\
\hline & chisel & 555 & 231 & 38.00 & 1.749 & \\
\hline & chisel & 560 & 241 & 37.34 & 1.751 & \\
\hline & chisel & 650 & 286 & 38.52 & 1.740 & \\
\hline
\end{tabular}




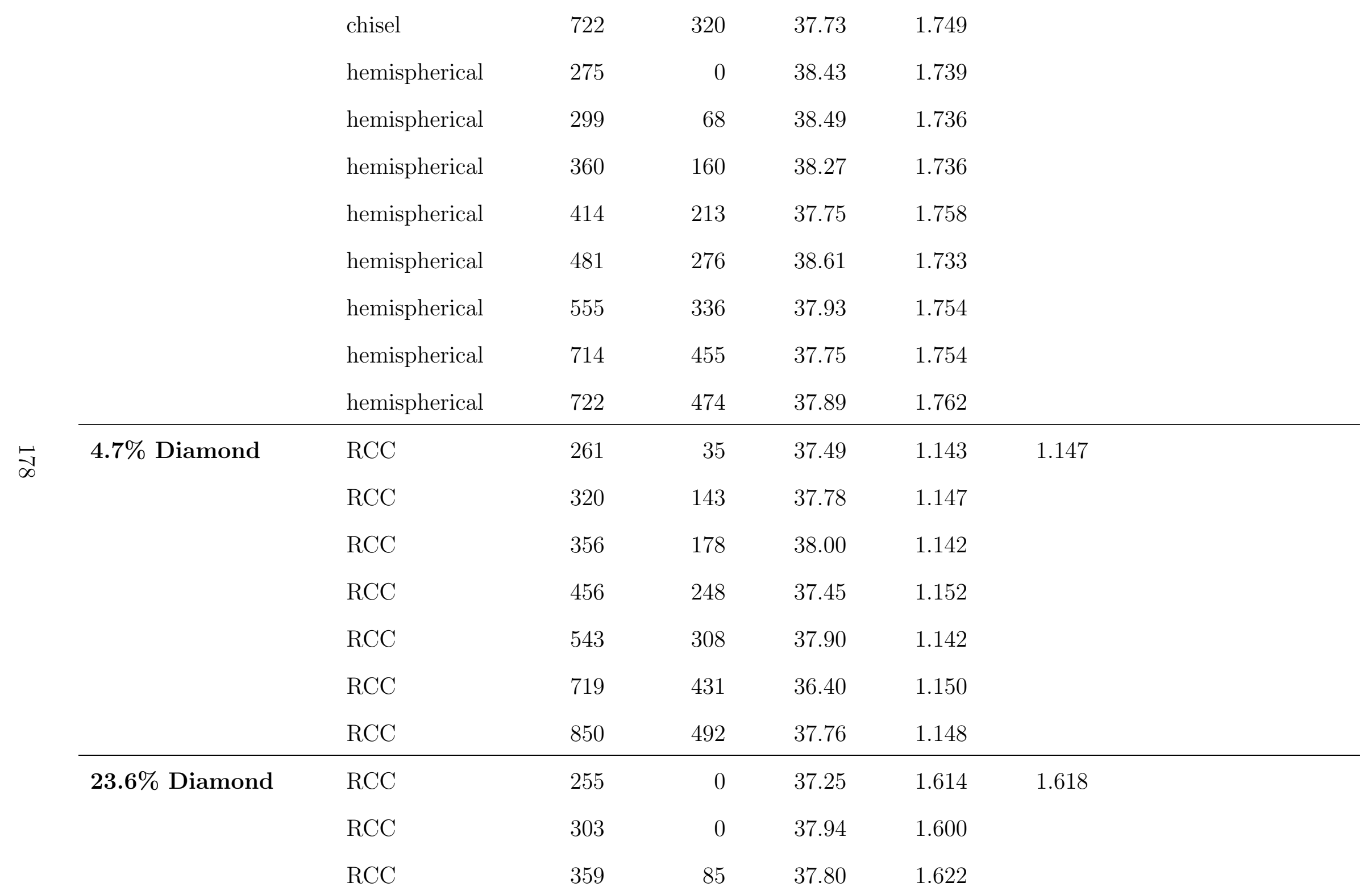




\begin{tabular}{|c|c|c|c|c|c|c|c|}
\hline & $\mathrm{RCC}$ & 410 & 123 & 38.04 & 1.623 & & \\
\hline & $\mathrm{RCC}$ & 535 & 208 & 37.62 & 1.621 & & \\
\hline & $\mathrm{RCC}$ & 731 & 325 & 37.80 & 1.616 & & \\
\hline & $\mathrm{RCC}$ & 878 & 361 & 37.75 & 1.624 & & \\
\hline \multirow[t]{6}{*}{ 33.3\% Diamond } & $\mathrm{RCC}$ & 349 & 0 & 38.33 & 1.825 & \multirow[t]{6}{*}{1.859} & Diff. diamond supplier \\
\hline & $\mathrm{RCC}$ & 374 & 49 & 38.10 & 1.843 & & Diff. diamond supplier \\
\hline & $\mathrm{RCC}$ & 445 & 115 & 38.39 & 1.821 & & Diff. diamond supplier \\
\hline & $\mathrm{RCC}$ & 550 & 187 & 37.83 & 1.845 & & Diff. diamond supplier \\
\hline & $\mathrm{RCC}$ & 651 & 227 & 38.07 & 1.842 & & Diff. diamond supplier \\
\hline & $\mathrm{RCC}$ & 709 & 245 & 38.31 & 1.825 & & Diff. diamond supplier \\
\hline \multirow{9}{*}{$\begin{array}{l}33.3 \% \mathrm{Al}_{2} \mathrm{O}_{3} \\
\text { (irregular) }\end{array}$} & $\mathrm{RCC}$ & 354 & 0 & 38.01 & 2.023 & \multirow[t]{9}{*}{2.002} & \multirow{9}{*}{ Projectile yaw } \\
\hline & $\mathrm{RCC}$ & 361 & 0 & 37.90 & 1.969 & & \\
\hline & $\mathrm{RCC}$ & 408 & 0 & 38.39 & 1.958 & & \\
\hline & $\mathrm{RCC}$ & 456 & 66 & 38.12 & 1.967 & & \\
\hline & $\mathrm{RCC}$ & 515 & 114 & 38.16 & 1.974 & & \\
\hline & $\mathrm{RCC}$ & 563 & 160 & 37.70 & 2.005 & & \\
\hline & $\mathrm{RCC}$ & 711 & 203 & 37.89 & 2.004 & & \\
\hline & conical & 564 & 231 & 37.75 & 1.999 & & \\
\hline & chisel & 356 & 0 & 38.56 & 2.007 & & \\
\hline
\end{tabular}




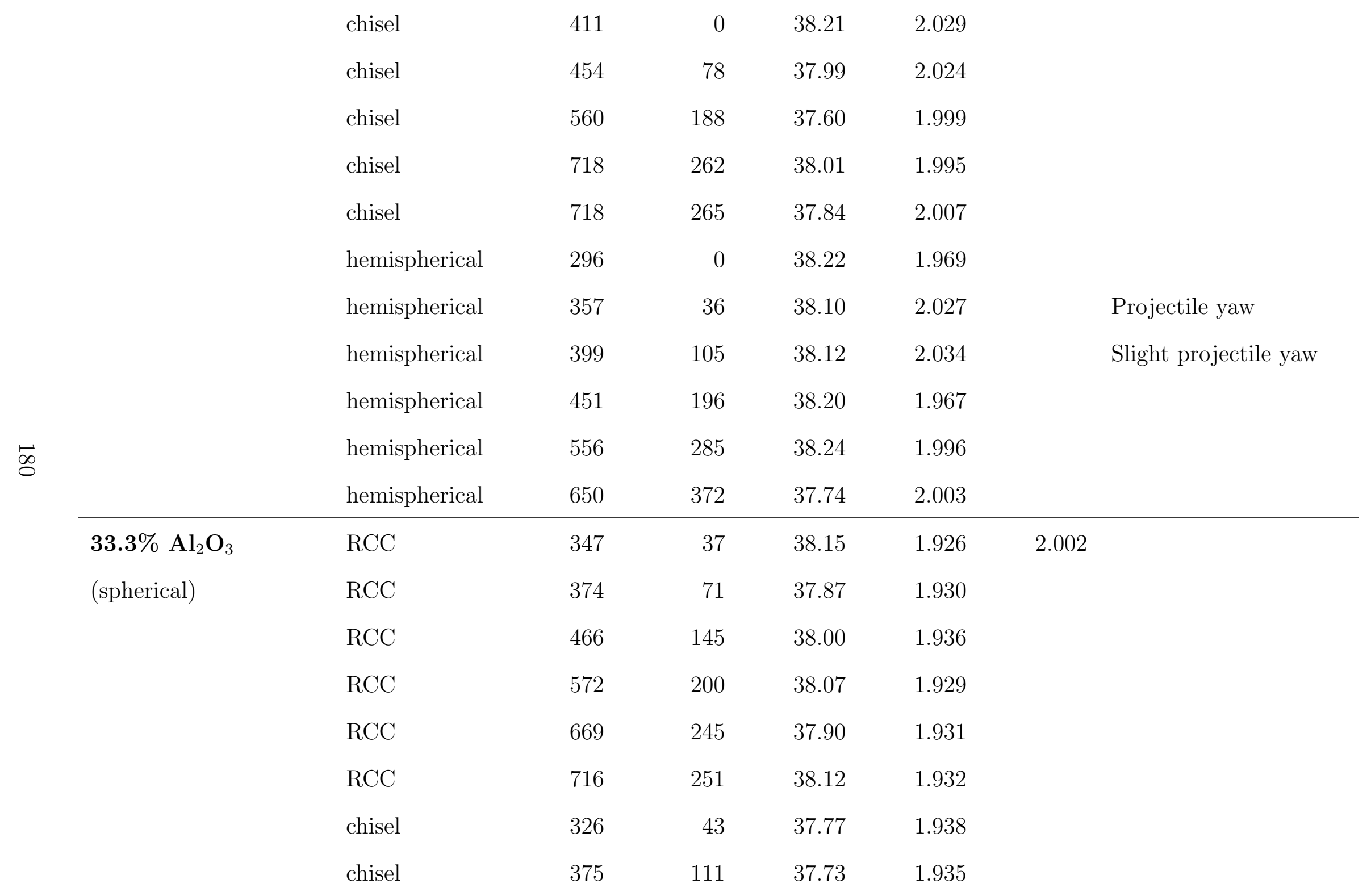




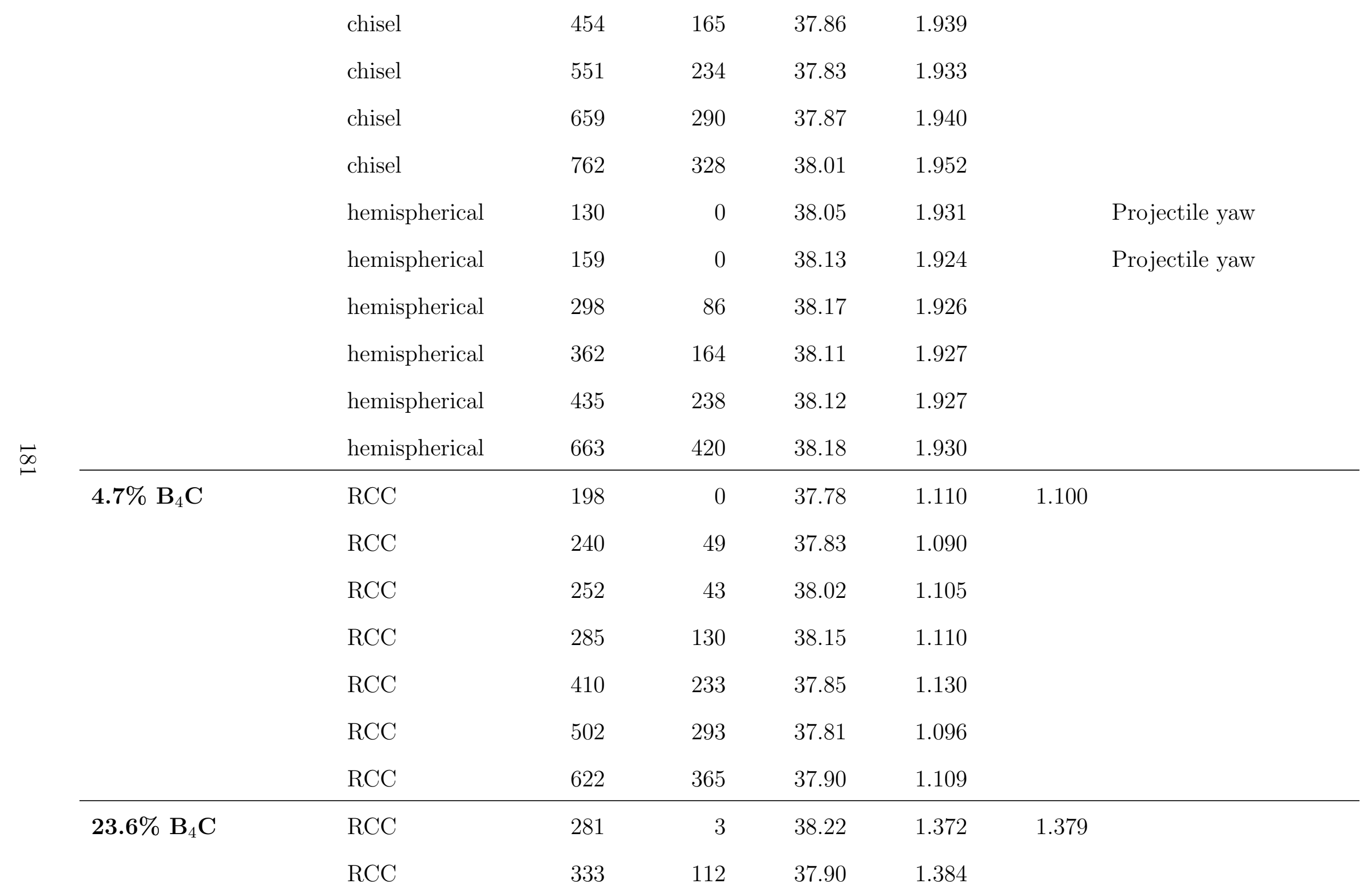




\begin{tabular}{|c|c|c|c|c|c|c|c|}
\hline & $\mathrm{RCC}$ & 389 & 158 & 38.00 & 1.377 & & \\
\hline & $\mathrm{RCC}$ & 420 & 183 & 37.92 & 1.380 & & \\
\hline & $\mathrm{RCC}$ & 556 & 265 & 37.63 & 1.382 & & \\
\hline & $\mathrm{RCC}$ & 715 & 356 & 38.29 & 1.356 & & \\
\hline & $\mathrm{RCC}$ & 863 & 431 & 37.95 & 1.374 & & \\
\hline $33.3 \% \mathrm{~B}_{4} \mathrm{C}$ & $\mathrm{RCC}$ & 317 & 0 & 38.16 & 1.506 & 1.523 & Projectile yaw \\
\hline & $\mathrm{RCC}$ & 401 & 109 & 38.32 & 1.496 & & \\
\hline & $\mathrm{RCC}$ & 526 & 154 & 38.49 & 1.497 & & \\
\hline & $\mathrm{RCC}$ & 633 & 270 & 37.74 & 1.513 & & \\
\hline & $\mathrm{RCC}$ & 711 & 306 & 38.30 & 1.501 & & \\
\hline $28 \% \mathrm{SiO}_{2}$ & $\mathrm{RCC}$ & 207 & 0 & 38.57 & 1.287 & 1.260 & \\
\hline & $\mathrm{RCC}$ & 352 & 133 & 38.56 & 1.285 & & \\
\hline & $\mathrm{RCC}$ & 446 & 197 & 38.90 & 1.280 & & \\
\hline & $\mathrm{RCC}$ & 534 & 273 & 38.27 & 1.276 & & \\
\hline & $\mathrm{RCC}$ & 612 & 326 & 38.15 & 1.272 & & \\
\hline & $\mathrm{RCC}$ & 674 & 350 & 38.89 & 1.285 & & \\
\hline $33.3 \% \mathrm{~B}_{4} \mathrm{C}$ & chisel & 341 & 0 & 44.16 & 1.504 & 1.523 & \\
\hline (const. $A_{d}$ ) & chisel & 405 & 95 & 43.95 & 1.505 & & \\
\hline & chisel & 455 & 136 & 44.39 & 1.500 & & \\
\hline
\end{tabular}




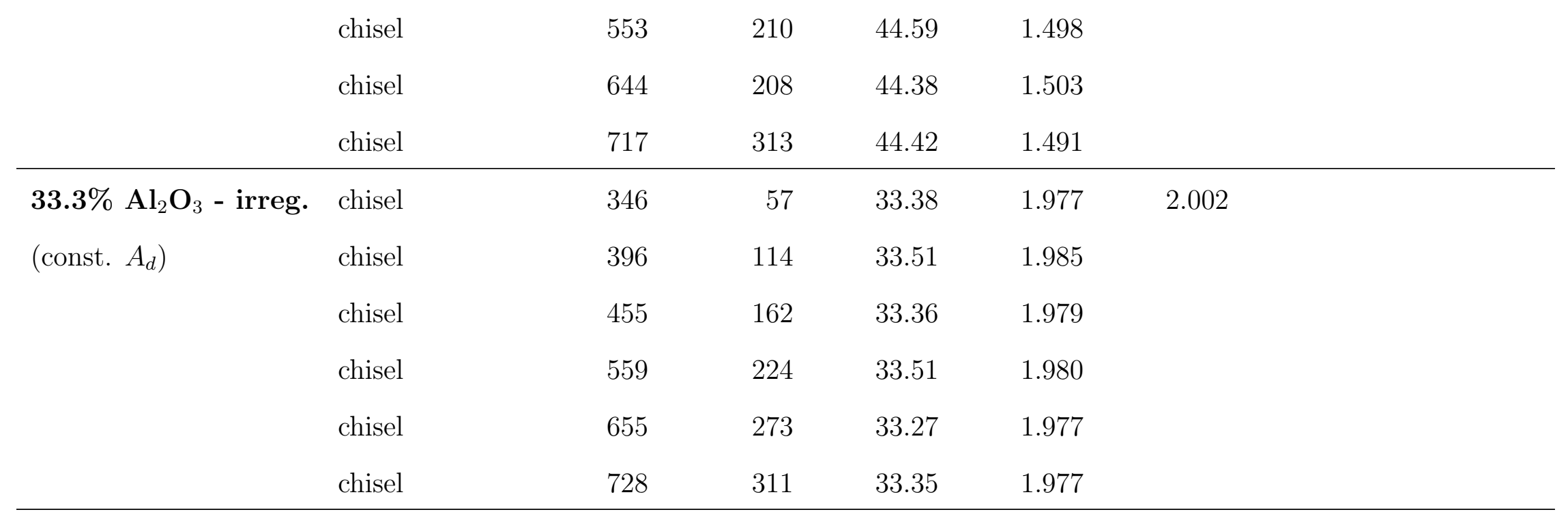

$\underset{\infty}{\infty}$ 


\section{B Estimated Ballistic Limit Velocities}

The ballistic limit velocities have been estimated for every target-projectile combination by approximating the horizontal intercept value of $V_{r}-V_{i}$ plots, based on the available datasets.

Table 9. Estimated ballistic limit values $\mathrm{V}_{b l}(\mathrm{~m} / \mathrm{s})$ for several target-projectile combinations.

\begin{tabular}{|l|c|c|c|}
\cline { 2 - 4 } \multicolumn{1}{c|}{} & \multicolumn{3}{c|}{ Projectile nose geometry } \\
\hline MDMS & RCC & Chisel-nosed & Hemispherical \\
4.7 vol.\% B ${ }_{4} \mathrm{C}$ & 195 & 220 & 180 \\
4.7 vol.\% SiC & 225 & - & - \\
4.7 vol.\% Diamond & 245 & 190 & - \\
23.6 vol.\% $\mathrm{B}_{4} \mathrm{C}$ & 250 & - & - \\
23.6 vol.\% SiC & 280 & - & - \\
23.6 vol.\% Diamond & 315 & 271 & - \\
33.3 vol.\% $\mathrm{B}_{4} \mathrm{C}$ & 325 & - & - \\
33.3 vol.\% $\mathrm{SiC}$ & 337 & 320 & - \\
33.3 vol.\% Diamond & 349 & - & - \\
33.3 vol.\% $\mathrm{Al}_{2} \mathrm{O}_{3}$ - irreg. & 415 & 411 & - \\
33.3 vol.\% $\mathrm{Al}_{2} \mathrm{O}_{3}$ - spher. & 325 & 305 & - \\
28.0 vol.\% $\mathrm{SiO}_{2}$ & 250 & - & - \\
33.3 vol.\% $\mathrm{B}_{4} \mathrm{C}$ (const. $\left.A_{d}\right)$ & - & 350 & - \\
33.3 vol.\% $\mathrm{Al}_{2} \mathrm{O}_{3}$ - irreg. (const. $\left.A_{d}\right)$ & - & 315 & - \\
\hline
\end{tabular}




\section{Additional Images}

Figures that did not entirely add crucial information to the discussions were moved to Appendix C.1. The damaged surfaces and penetration channels resulting from ballistic capsule experiments have been photographed and organized in Appendix C.2 and C.3, respectively, to obtain a compact collection of images for comparison purposes. The capsules were selected for imaging in order to illustrate the effects of particle volume fraction, material, impact velocity, and projectile nose geometry.

\section{C.1 Miscellaneous}

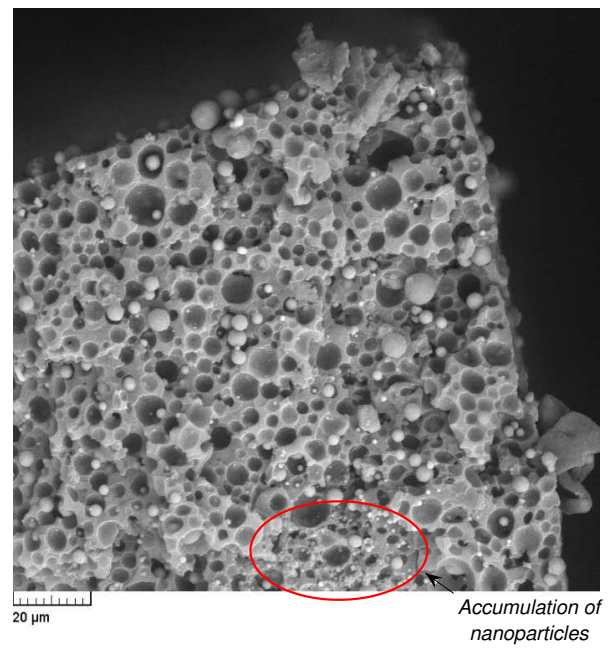

(a)

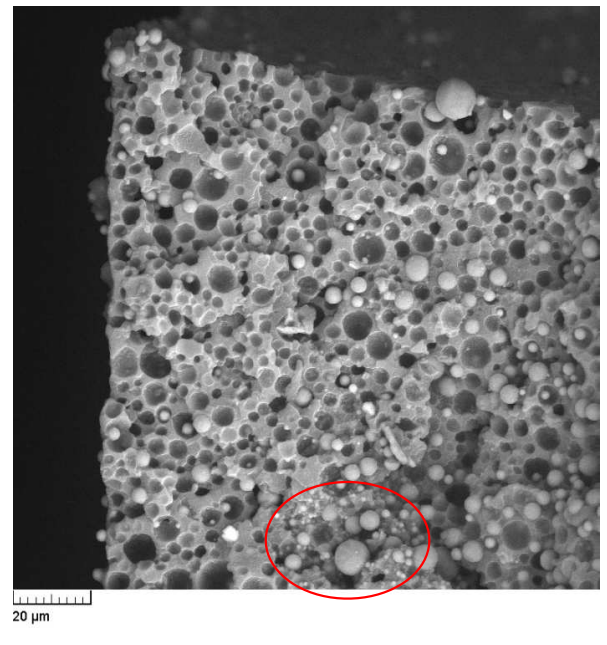

(b)

Figure 109. Tensile failure surface (specimen corner) of $33.3 \%$ spherical $\mathrm{Al}_{2} \mathrm{O}_{3}$-PDMS showing matching interfaces along with cluster of nanoparticles (red circle). 


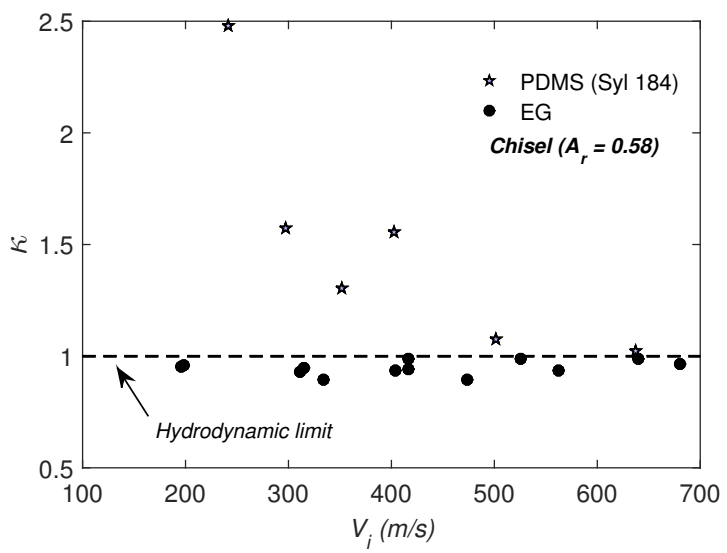

Figure 110. Ballistic performance parameter for PDMS and EG capsules. Data for EG was extracted from Petel et al. [44].

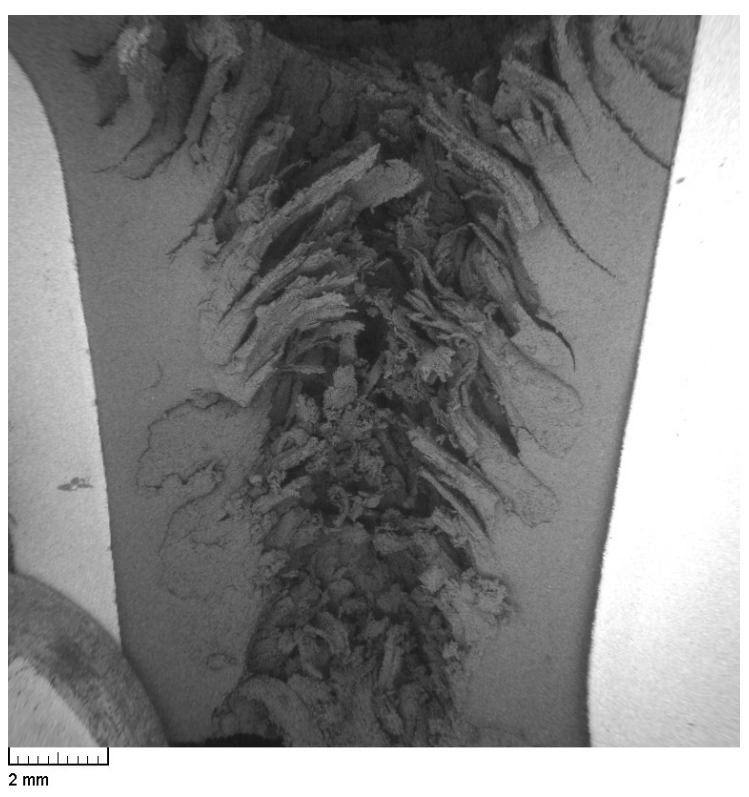

(a)

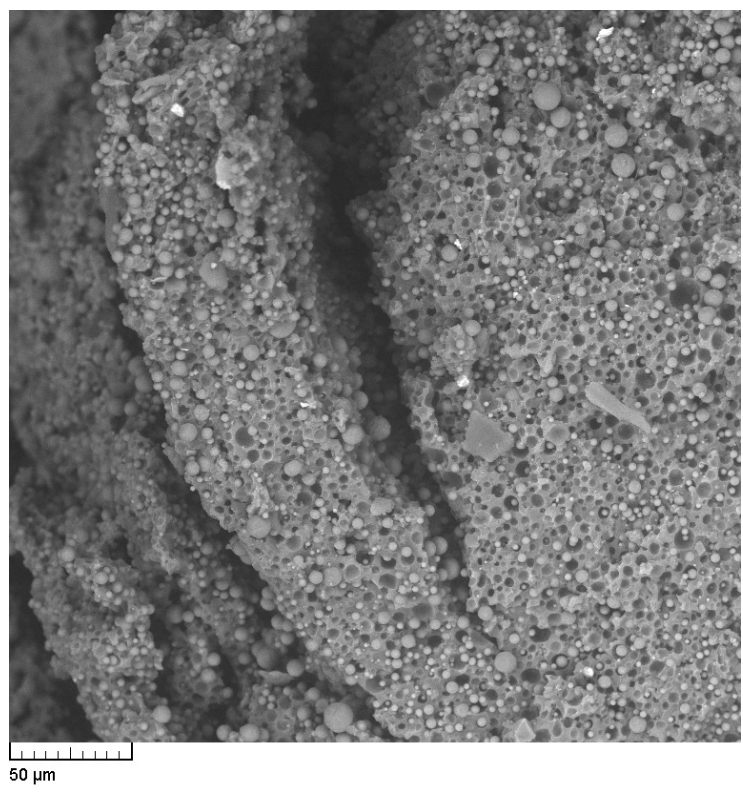

(b)

Figure 111. SEM images of 33.3 vol.\% spherical $\mathrm{Al}_{2} \mathrm{O}_{3}$-PDMS penetration channel: (a) overall and (b) magnification. 


\section{C.2 Damaged Planes of Ballistic Capsules}

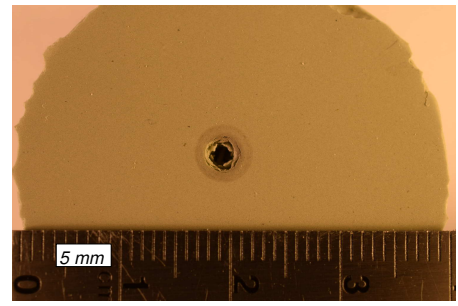

(a)

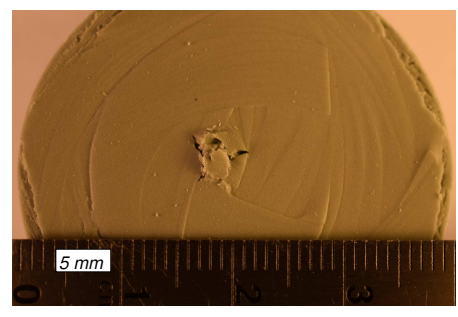

(c)

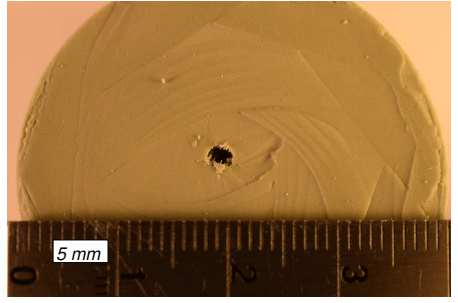

(b)

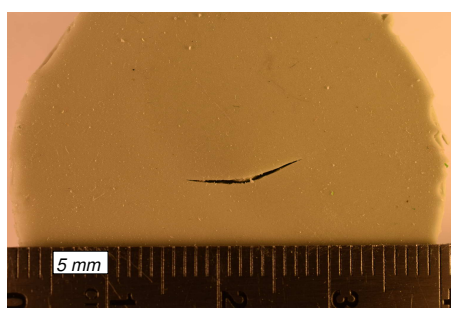

(d)

Figure 112. Damaged planes - 4.7 vol. $\%$ SiC-PDMS (projectile: RCC) $-V_{i}=257 \mathrm{~m} / \mathrm{s}$ (slightly above $V_{b l}$ ): (a) impact surface, (b) through-thickness surface $t_{r e l}=0.33$, (c) $t_{r e l}=$ 0.67, and (d) back surface. Direction of impact: into the page except for (d).

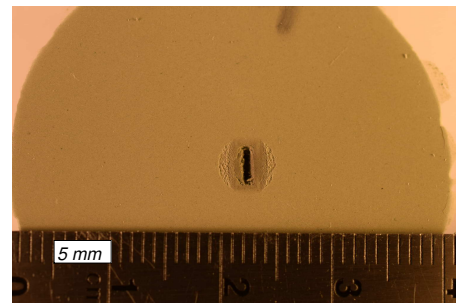

(a)

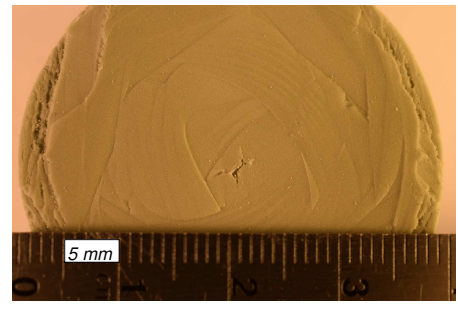

(c)

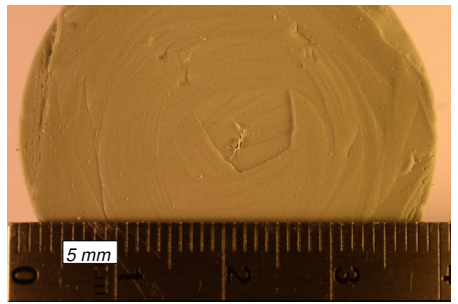

(b)

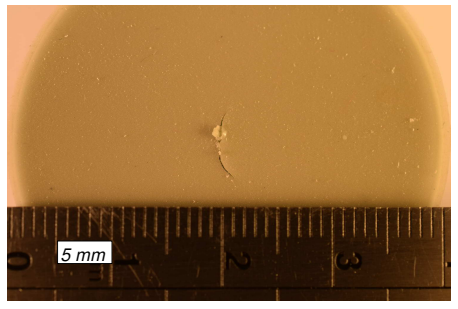

(d)

Figure 113. Damaged planes -4.7 vol.\% SiC-PDMS (projectile: chisel-nosed) $-V_{i}=229$ $\mathrm{m} / \mathrm{s}$ (slightly above $V_{b l}$ ): (a) impact surface, (b) through-thickness surface $t_{r e l}=0.33$, (c) $t_{r e l}=0.67$, and (d) back surface. Direction of impact: into the page except for $(\mathrm{d})$. 


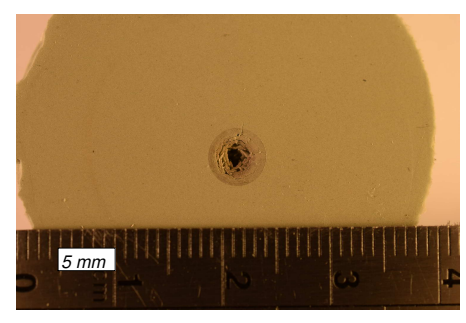

(a)

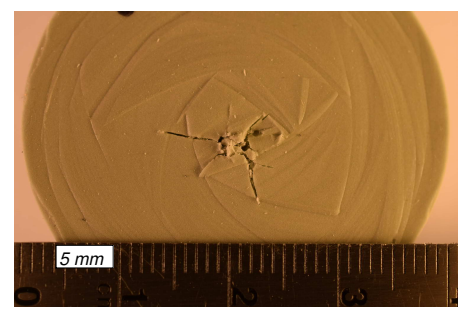

(c)

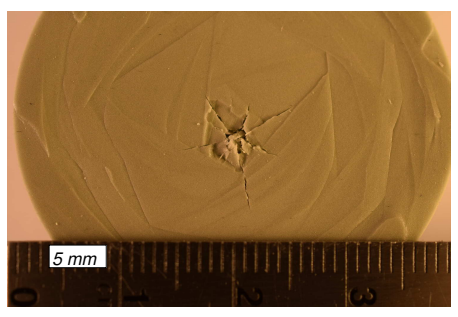

(b)

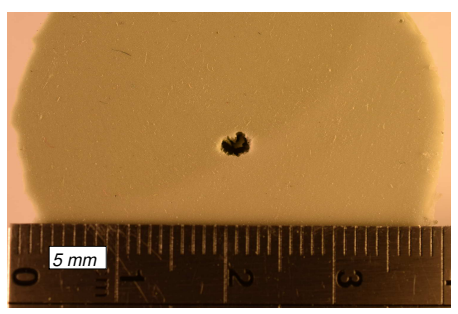

(d)

Figure 114. Damaged planes -4.7 vol.\% SiC-PDMS (projectile: RCC) $-V_{i}=430 \mathrm{~m} / \mathrm{s}$ : (a) impact surface, (b) through-thickness surface $t_{r e l}=0.33$, (c) $t_{r e l}=0.67$, and (d) back surface. Direction of impact: into the page except for $(d)$.

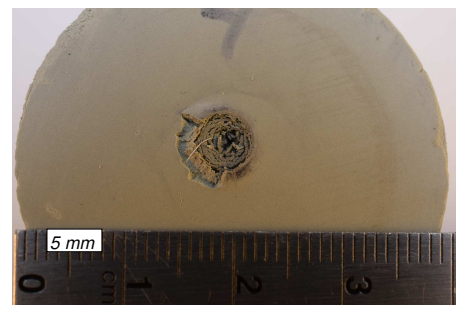

(a)

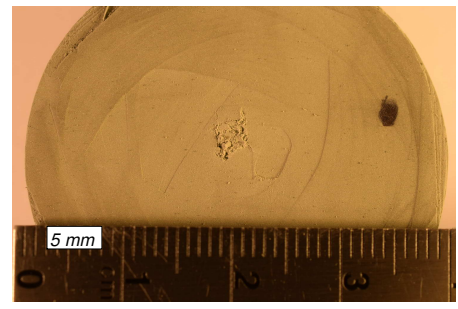

(c)

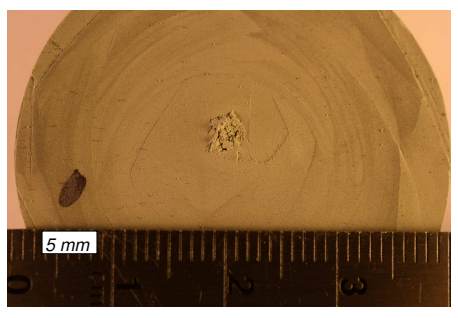

(b)

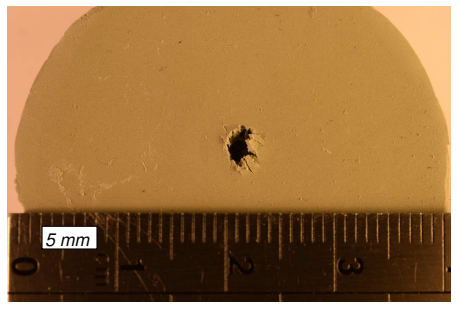

(d)

Figure 115. Damaged planes -33.3 vol.\% SiC-PDMS (projectile: $\mathrm{RCC}$ ) $-V_{i}=423 \mathrm{~m} / \mathrm{s}$ : (a) impact surface, (b) through-thickness surface $t_{r e l}=0.33$, (c) $t_{r e l}=0.67$, and (d) back surface. Direction of impact: into the page except for $(d)$. 


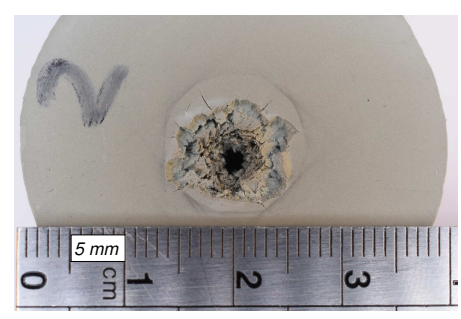

(a)

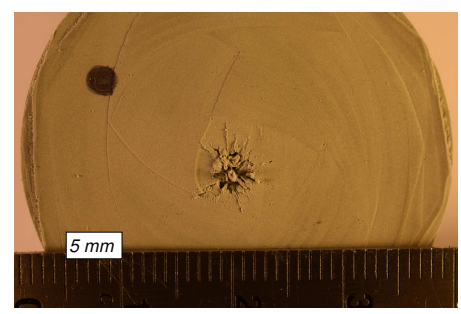

(c)

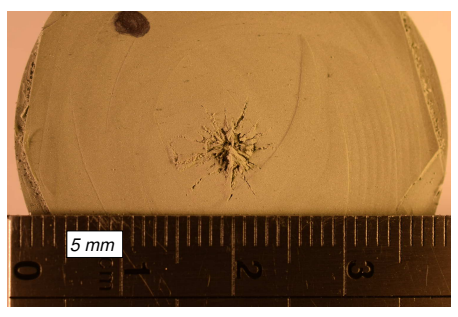

(b)

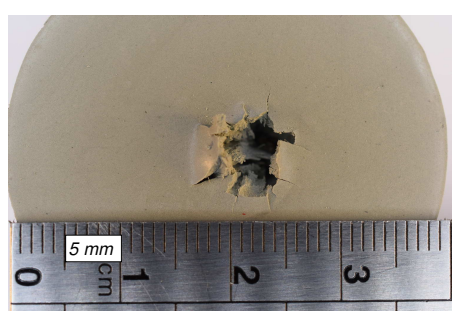

(d)

Figure 116. Damaged planes - 33.3 vol. $\%$ SiC-PDMS (projectile: $\mathrm{RCC}$ ) $-V_{i}=740 \mathrm{~m} / \mathrm{s}$ : (a) impact surface, (b) through-thickness surface $t_{r e l}=0.33$, (c) $t_{r e l}=0.67$, and (d) back surface. Direction of impact: into the page except for (d).

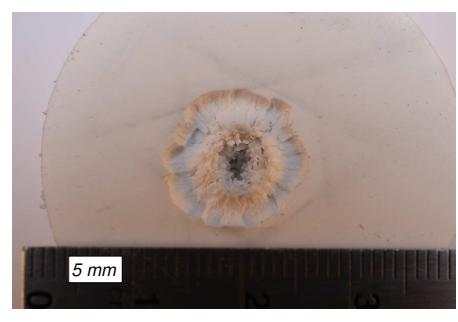

(a)

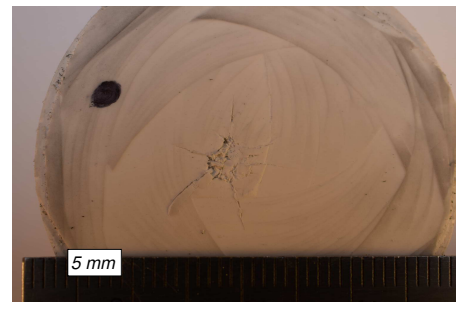

(c)

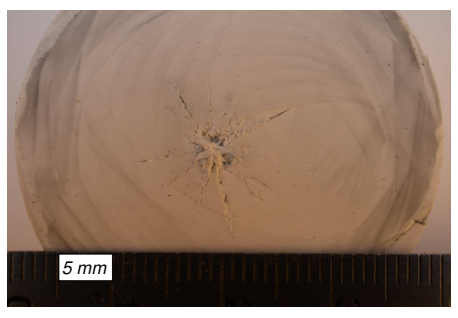

(b)

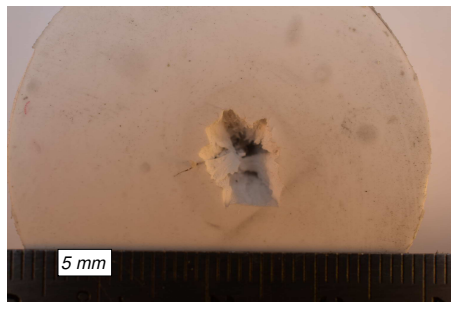

(d)

Figure 117. Damaged planes - 33.3 vol. $\% \mathrm{Al}_{2} \mathrm{O}_{3}$-PDMS (projectile: $\mathrm{RCC}$ ) $-V_{i}=711 \mathrm{~m} / \mathrm{s}$ : (a) impact surface, (b) through-thickness surface $t_{r e l}=0.33$, (c) $t_{r e l}=0.67$, and (d) back surface. Direction of impact: into the page except for (d). 


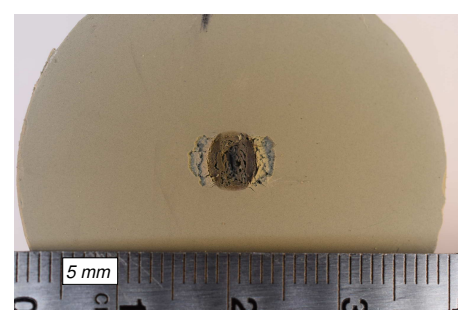

(a)

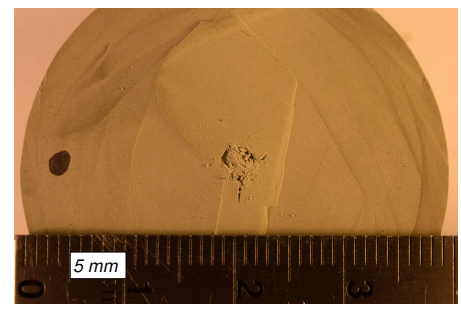

(c)

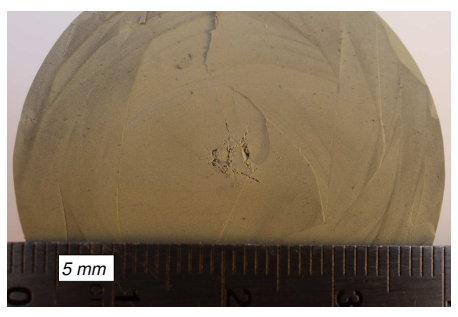

(b)

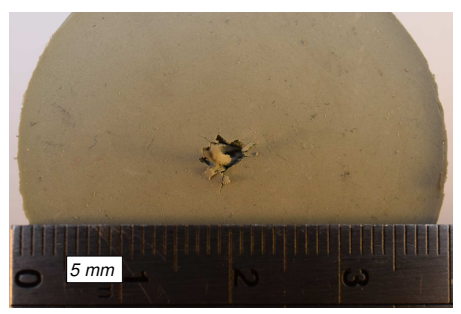

(d)

Figure 118. Damaged planes - 33.3 vol.\% SiC-PDMS (projectile: chisel-nosed) $-V_{i}=392$ $\mathrm{m} / \mathrm{s}$ : (a) impact surface, (b) through-thickness surface $t_{\text {rel }}=0.33$, (c) $t_{\text {rel }}=0.67$, and (d) back surface. Direction of impact: into the page except for (d).

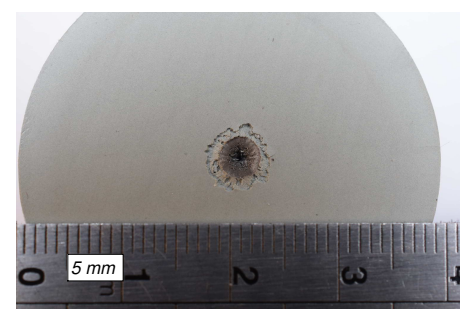

(a)

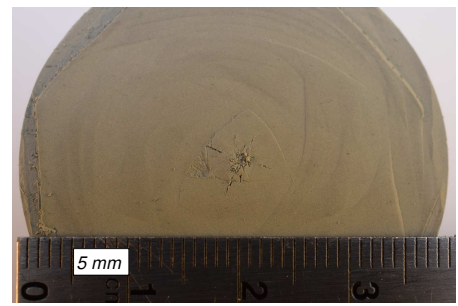

(c)

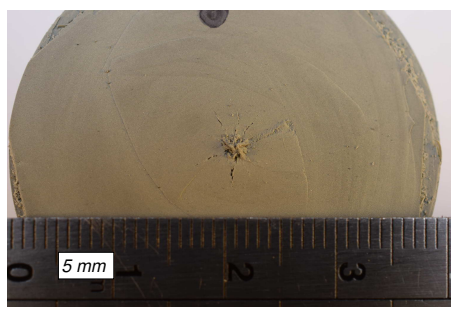

(b)

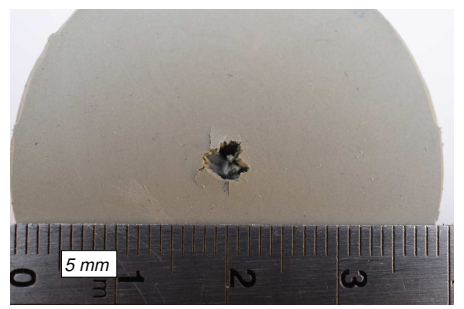

(d)

Figure 119. Damaged planes - 33.3 vol.\% SiC-PDMS (projectile: hemispherical) $-V_{i}=414$ $\mathrm{m} / \mathrm{s}$ : (a) impact surface, (b) through-thickness surface $t_{r e l}=0.33$, (c) $t_{r e l}=0.67$, and (d) back surface. Direction of impact: into the page except for $(\mathrm{d})$. 


\section{C.3 Penetration Channels of Ballistic Capsules}

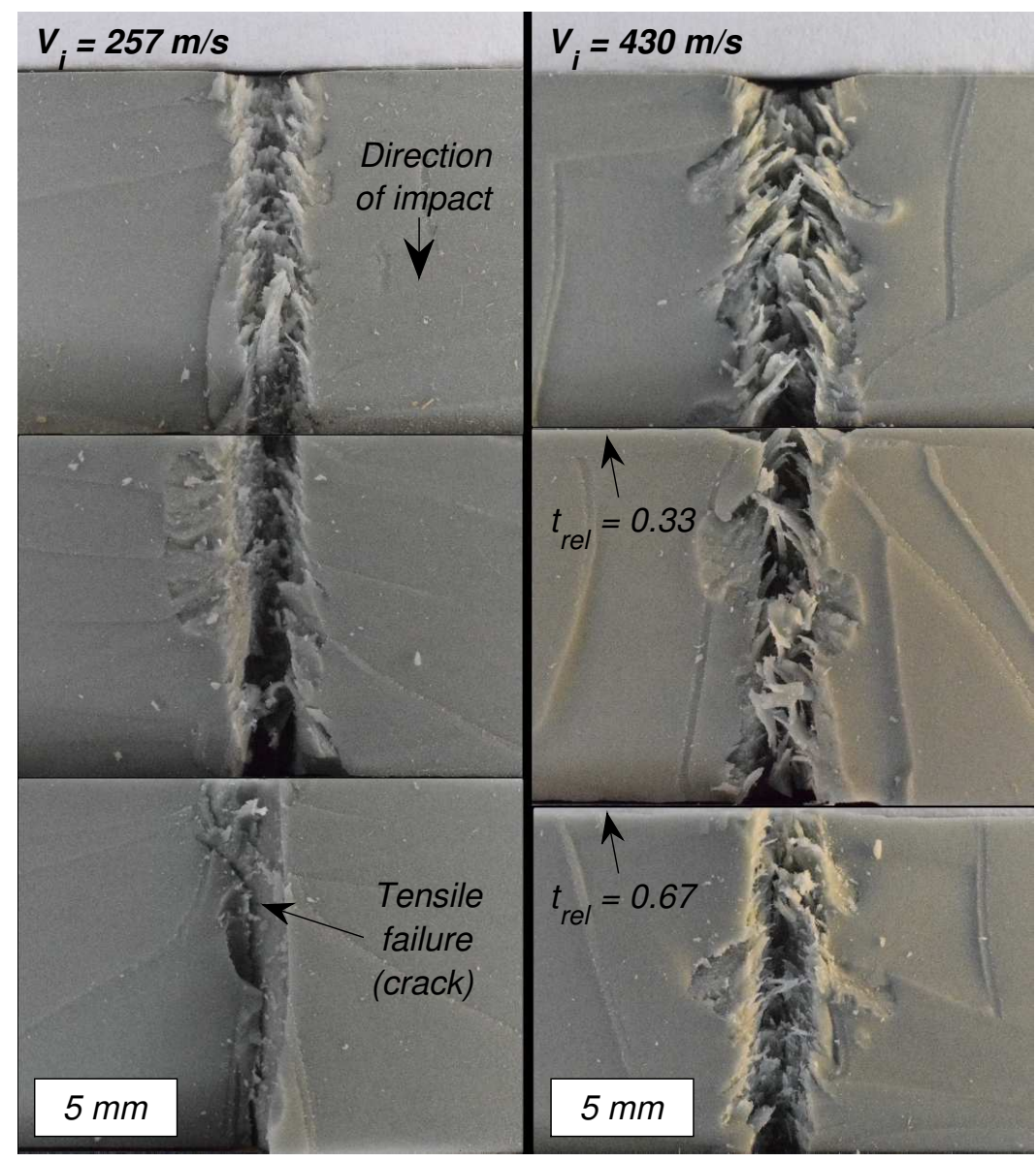

(a)

(b)

Figure 120. Stitched images of entire penetration channels of 4.7 vol.\% SiC-PDMS capsules (projectile: RCC) showing effect of impact velocity: (a) $V_{i}=257 \mathrm{~m} / \mathrm{s}$ and (b) $V_{i}=430 \mathrm{~m} / \mathrm{s}$. Impact and exit surfaces at top and bottom of images, respectively. 


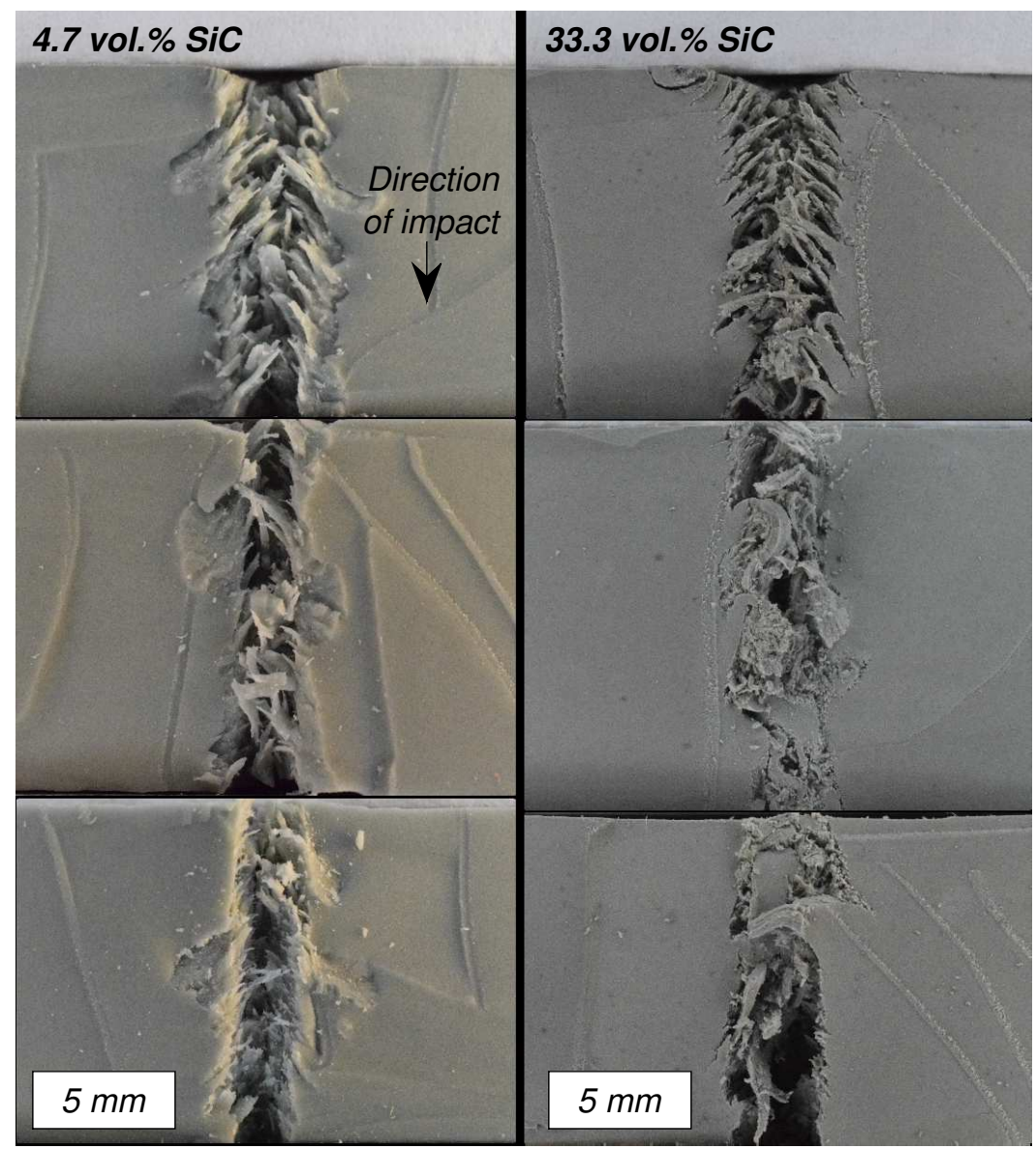

(a)

(b)

Figure 121. Stitched images of entire penetration channels of SiC-PDMS capsules (projectile: RCC) showing effect of particle volume fraction: (a) $V_{i}=430 \mathrm{~m} / \mathrm{s}$ and (b) $V_{i}=423$ $\mathrm{m} / \mathrm{s}$. Impact and exit surfaces at top and bottom of images, respectively. 


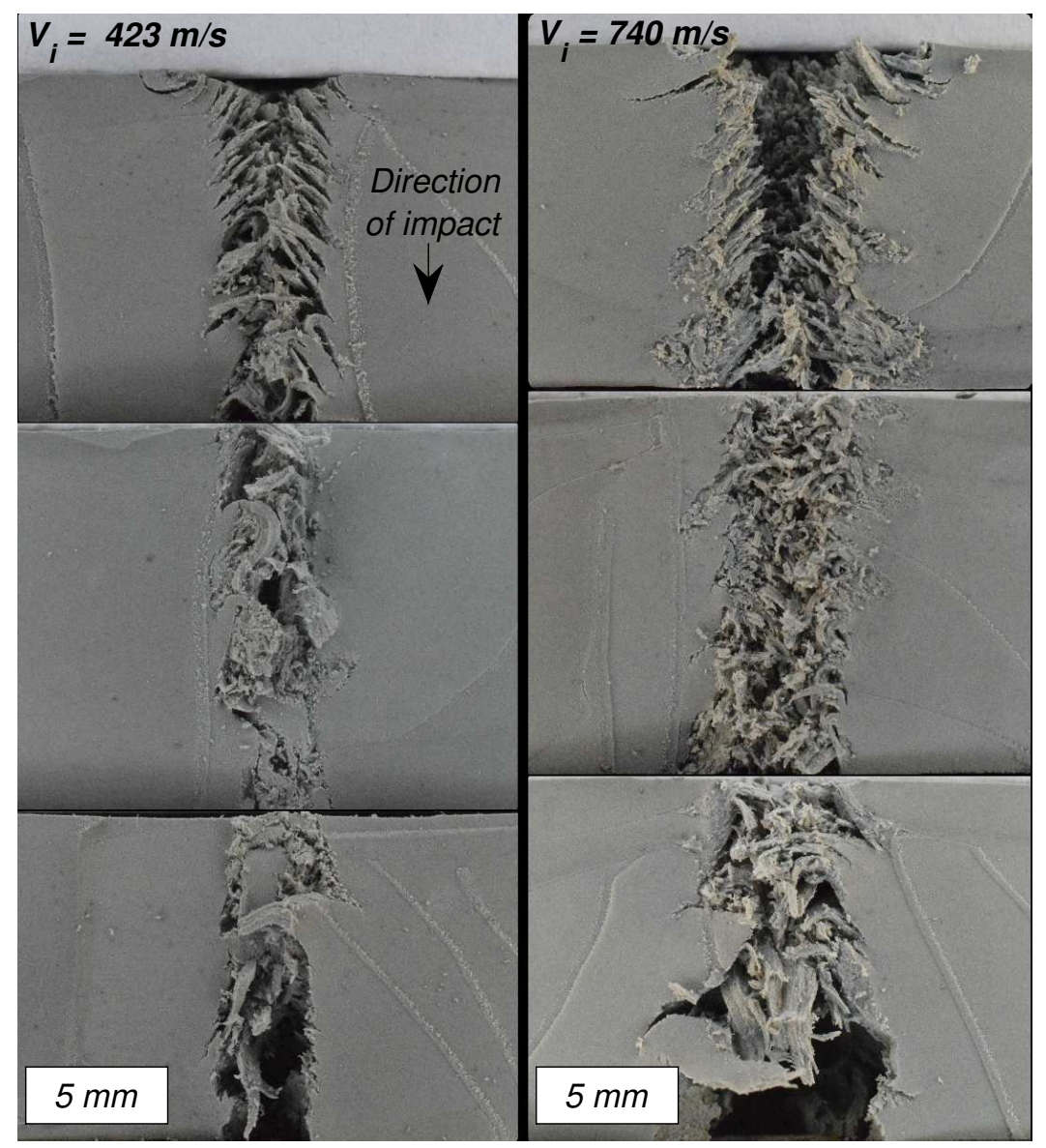

(a) (b)

Figure 122. Stitched images of entire penetration channels of 33.3 vol.\% SiC-PDMS capsules (projectile: RCC) showing effect of impact velocity: (a) $V_{i}=423 \mathrm{~m} / \mathrm{s}$ and (b) $V_{i}=740$ $\mathrm{m} / \mathrm{s}$. Impact and exit surfaces at top and bottom of images, respectively. 


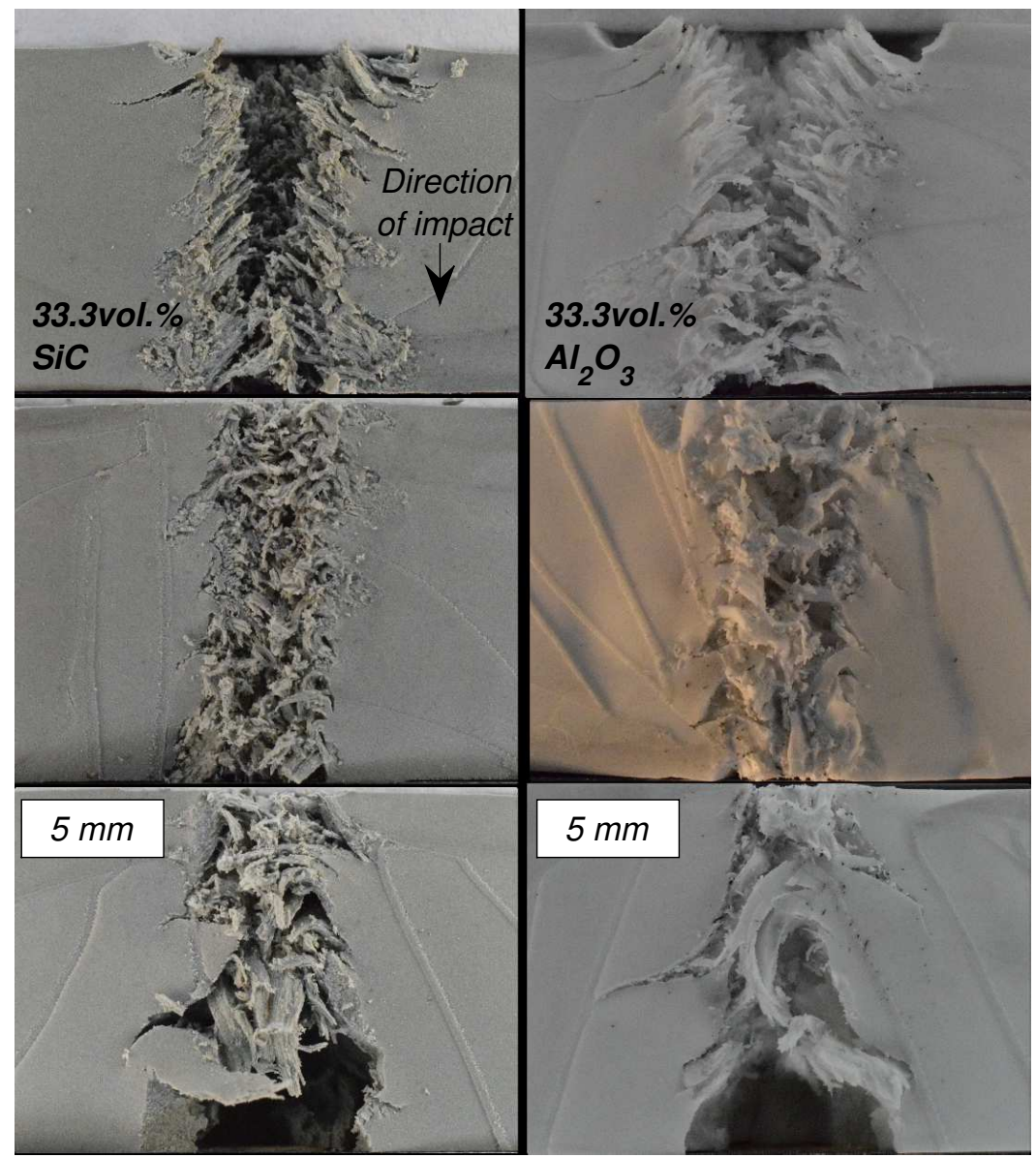

(a)

(b)

Figure 123. Stitched images of entire penetration channels of 33.3 vol.\% particulate composites (projectile: RCC) showing effect of particle material type: (a) SiC-PDMS, $V_{i}=740$ $\mathrm{m} / \mathrm{s}$ and (b) $\mathrm{Al}_{2} \mathrm{O}_{3}-\mathrm{PDMS}, V_{i}=711 \mathrm{~m} / \mathrm{s}$. Impact and exit surfaces at top and bottom of images, respectively. 


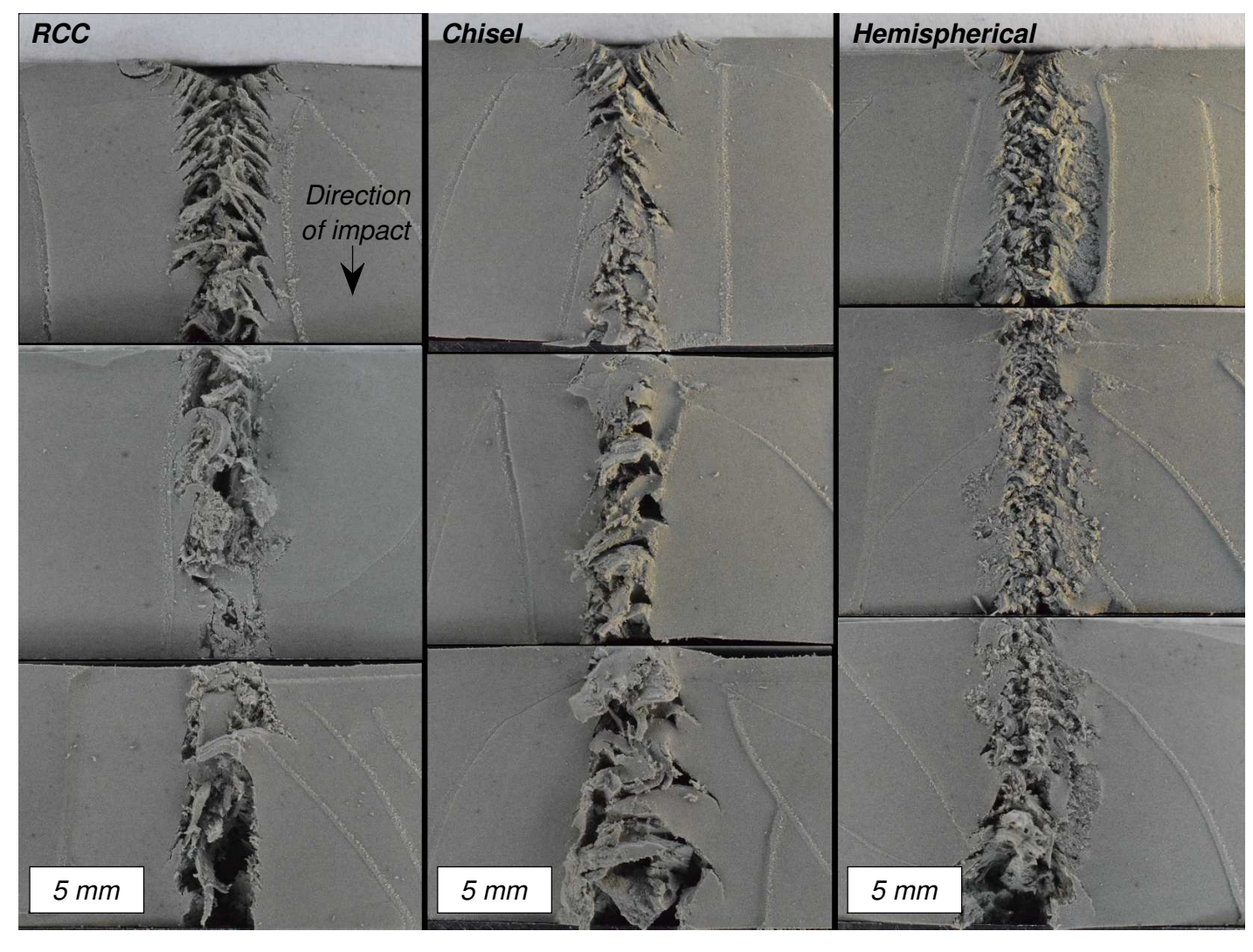

(a)

(b)

(c)

Figure 124. Stitched images of entire penetration channels of 33.3 vol.\% SiC-PDMS capsules impacted by various projectile nose geometries: (a) RCC, $V_{i}=423 \mathrm{~m} / \mathrm{s}$, (b) chiselnosed, $V_{i}=392 \mathrm{~m} / \mathrm{s}$, and (c) hemispherical, $V_{i}=414 \mathrm{~m} / \mathrm{s}$. Impact and exit surfaces at top and bottom of images, respectively. 